\title{
Towards high electron mobility in GaN (0001) based InGaN and AlGaN heterostructures
}

\author{
Dissertation \\ zur Erlangung des mathematisch-naturwissenschaftlichen Doktorgrades \\ "‘Doctor rerum naturalium"' \\ der Georg-August-Universität Göttingen
}

\author{
vorgelegt von \\ Daniel Broxtermann \\ aus Georgsmarienhütte
}

Göttingen, 2011 


\section{Referentin:}

Prof. Dr. Angela Rizzi

Korreferent:

Prof. Dr. Andreas Hangleiter

Tag der mündlichen Prüfung: 28.10.2011 
Dedicated to my family. 
This page intentionally left blank.

There is absolutely no text here. 
CONTENTS

I BACKGROUND OF EXPERIMENTAL METHODS

1 MOLECULAR BEAM EPITAXY OF III-NITRIDES

1.1 Growth of III-nitrides . . . . . . . . . . . . 7

1.1.1 Historic development . . . . . . . . . . 7

1.1.2 MBE setup ................. 8

1.1.3 Advantages and challenges of III-N growth by MBE . 11

1.2 Surface processes during MBE growth ............ 13

1.3 Two dimensional growth modes . . . . . . . . . . . . 17

1.4 Gallium and indium bilayer . . . . . . . . . . . 23

1.5 MBE growth conditions for III-nitrides . . . . . . . . . . . 25

1.5.1 Substrates.................... 26

$1.5 .2 \mathrm{GaN} \ldots \ldots \ldots \ldots \ldots \ldots . \ldots \ldots$

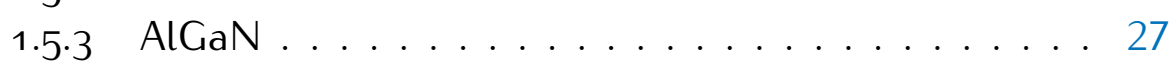

$1.5 .4 \operatorname{lnGaN} \ldots \ldots \ldots \ldots \ldots 29 \ldots \ldots \ldots$

2 RHEED

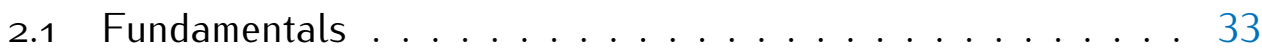

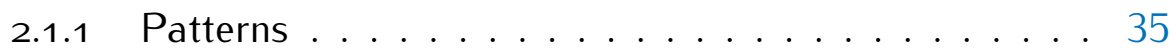

2.1 .2 Reconstructions . . . . . . . . . . . . 36

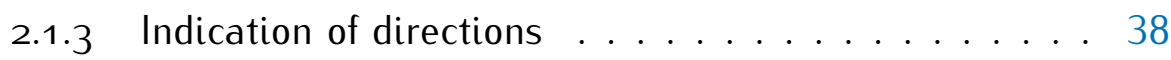

2.1.4 Spacing between reflexes . . . . . . . . . . . 39

2.2 Applications . . . . . . . . . . . . . . . . . . . 40

2.2.1 RRS: RHEED Radar Scans . . . . . . . . . . . . 40

2.2.2 Growth rate by RHEED oscillations . . . . . . . . . 40

2.2.3 Determination of stoichiometry and III/V ratios . . . . 42

2.2.4 Monitoring metal bilayer desorption ........ 43

3 TWO DIMENSIONAL ELECTRON TRANSPORT IN III-NITRIDES

3.1 2D carrier confinement in III-nitride heterostructures . . . . 45

3.2 Mobility and elastic scattering mechanisms . . . . . . . . 49

3.3 Hall effect: A brief reminder . . . . . . . . . . . . 52

3.4 Magnetoresistance . . . . . . . . . . . . . . 54

3.4.1 Shubnikov-de Haas oscillations .......... 55

3.4 .2 Weak localization ................... 58

3.4.3 Classical influence of scattering potentials . . . . . 60

SUMMARY OF THE BACKGROUND 65

II RESULTS OF GROWTH OPTIMIZATION

4 GALLIUM NITRIDE

4.1 Substrate pretreatment.................69 


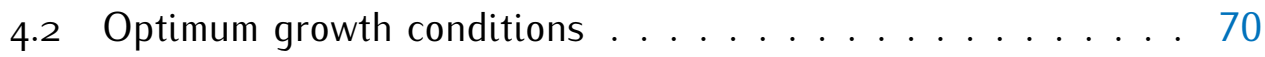

4.3 Modulated growth . . . . . . . . . . . . . . 74

5 ALUMINIUM GalliUm NitRide heterostructures

5.1 Growth conditions and structural quality . . . . . . . . . 77

5.2 GaN/AlGaN heterostructure design . . . . . . . . . . . . 79

5.3 Magnetoresistance ................. . . 82

5.4 Application: ionic and biochemical sensing . . . . . . . . . . 87

$5 \cdot 4.1$ Heterostructure modeling . . . . . . . . . . 89

5.4 .2 Experimental results ................... 91

6 INDIUM GALLIUM NITRIDE HETEROSTRUCTURES

6.1 Growth conditions and structural quality . . . . . . . . . 95

6.2 Surface degradation after indium desorption . . . . . . . . 100

6.3 Indium incorporation determined by RHEED . . . . . . . . . . . 104

6.4 Heterostructure design . . . . . . . . . . . . . . 106

6.5 Magnetotransport results . . . . . . . . . . . . . 109

SUMMARY OF OPTIMIZED GROWTH CONDITIONS 117

III CONCLUSION

7 SUMMARY, APPRECIATION AND OUTLOOK

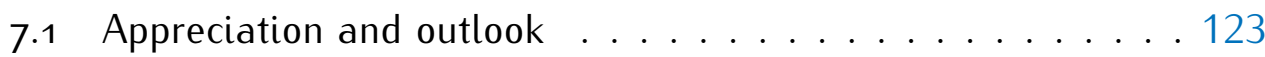

\section{LAB HANDBOOK}

A LETS GET GROWING

A.1 Welcome to the sample wash . . . . . . . . . . 127

A.2 Where stars are born: The growth chamber . . . . . . . . . . 128

A.3 GaN the Great . . . . . . . . . . . . . . . . . 129

A.4 AlGaN the Almighty . . . . . . . . . . . . . . . 131

A.5 InGaN the Terrible . . . . . . . . . . . . . 132

B RHEED 101

B.1 The homebrew software . . . . . . . . . . . . . 135

B.2 Smile: How to get a good image . . . . . . . . . . 137

C HOT OR NOT: ABSOLUTE SUBSTRATE TEMPERATURE

C.1 Old School: RHEED . . . . . . . . . . . . . . . . . . . . 140

c.2 New school: Band edge absorption . . . . . . . . . . . . 142 
Gallium nitride (GaN), aluminium nitride (AIN) and indium nitride $(\ln N)$ are members of the group III-nitrides (III-N), and are binary compounds of a group three metal and nitrogen. They have a wurtzite crystal structure and belong to the III-V compound semiconductors, which also include phosphides, arsenides and antimonides. In Figure 0.1 the band gap, which is the fundamental measure of semiconductors, is compared for all III-V semiconductors. Even at first glance it is clear, that III-N are remarkably versatile materials. All III-nitrides in the wurtzite structure have a direct band gap and cover the broadest band gap range in all compatible semiconductor systems. It spans from $6.2 \mathrm{eV}$ for AIN over $3.4 \mathrm{eV}$ for GaN down to $0.7 \mathrm{eV}$ for $\ln N$. The ternary compounds InGaN and AlGaN thus continuously cover wavelengths from the near infrared, over the entire visible spectrum up to far ultraviolet. It is foremost this property, but not the only, which has driven the research and development of this material system.

III-N devices are largely based on heterostructures, i.e one material is grown epitaxially on a different material. In many cases, interlayers of ternary III-N

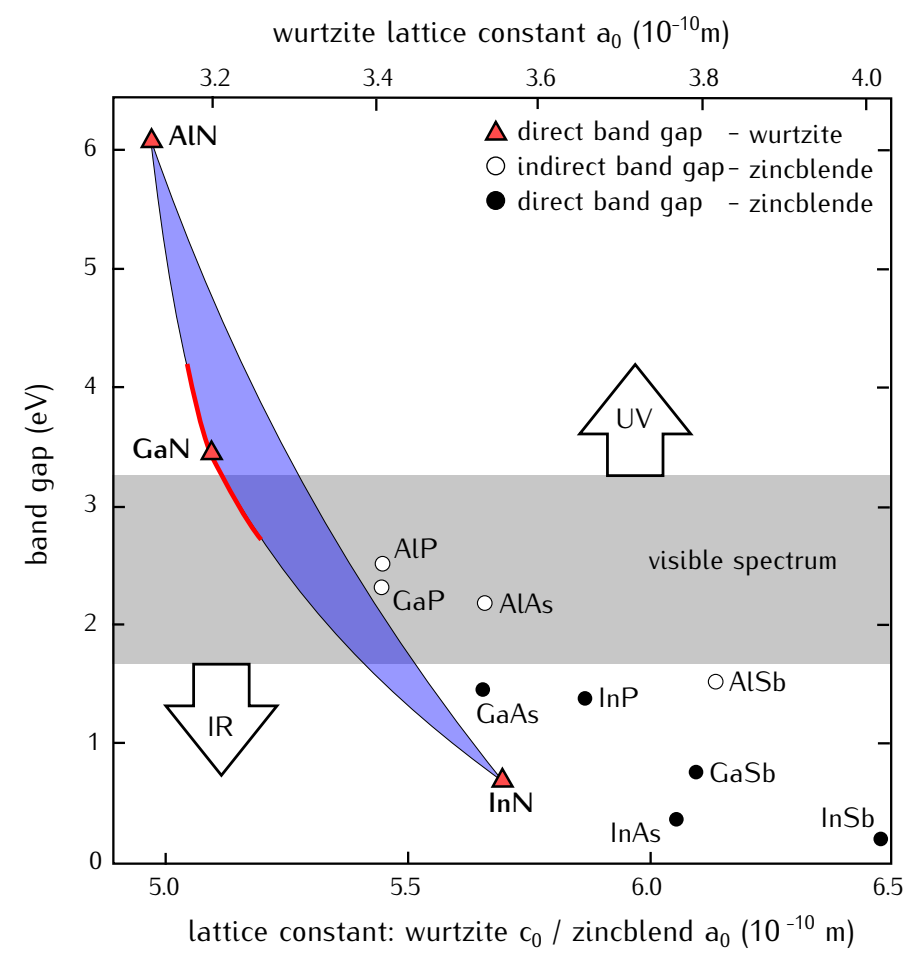

Figure 0.1: Band gaps of III-V compound semiconductors over lattice constants. The solid red line indicates the structures studied in this work. 
compounds are grown on GaN. This affects the band profile depending on the band gaps and the polarization of the different materials, giving rise to certain applications. In optoelectronics for instance, interlayers of materials with a smaller band gap result in wells which represent the active region for recombination. For transistors, the channel between source and drain is based on carriers accumulated at interfaces due to strong polarization fields. Today, the most prominent applications of III-N heterostructures are in optoelectronics. Primarily InGaN based devices are found in everyday items, such as laser diodes in Blu-ray players and blue or white LEDs, be it for lighting or as backlights in a TV, laptop or mobile phone. These fields of applications are based on the band gap in the visible range of InGaN.

Various AlGaN heterostructures devices are on the verge of commercial launch, because of their chemical properties in combination with the wide band gap. The bond strengths between Ga-N and $\mathrm{Al}-\mathrm{N}$ are high, which allows device operation in hazardous environments. Because of their high melting points above $2500{ }^{\circ} \mathrm{C}$ together with the wide band gap, they are suitable for high power and high temperature applications. In this segment, AlGaN heterostructure based devices are projected to gain a large market share in the near future. Furthermore, the materials are non toxic and are currently under investigation for applications as sensors in biology and medicine.

Anyone not familiar with the growth of these materials might assume, that the large number of operating and upcoming devices is the result of intensive research, which it is, and of high material quality, which it is not. The noble prize winner Wolfgang Pauli referred to semiconductor physics as dirt physics. In a letter in 1931 he wrote: One should not work on semiconductors, it is a mess, who knows if semiconductors even exist ${ }^{1}$. This statement addresses the sensitivity of semiconductors to impurities. Back then, all semiconductors contained a high number of defects, which compensated semi conducting features. Today, semiconductor physics can be rightfully considered highly clean because much attention is paid to clean environments for device production. Still, III-nitrides are subjected to a high amount of defects compared to other compound semiconductors, e.g. GaAs. This makes some basic properties hard to access.

This is especially true for InGaN. An example is the band gap of $\ln N$, and thus also InGaN, which was not determined precisely until recently. Optics are mostly apply to characterize this material system and little is known about the electrical transport properties. Electrical characterization of, e.g. the spin-orbit coupling, spin lifetimes or the effective mass in InGaN, would however be of great interest particularly with regard to possible applications in spin-transport devices. From AlGaN it is known, that in order to obtain these parameters from low-temperature measurements of two dimensional

1 Original: 'Über Halbleiter sollte man nicht arbeiten, das ist eine Schweinerei, wer weiß ob es überhaupt Halbleiter gibt' 
electron gases (2DEGs), the growth of high quality heterostructures with high electron mobilities is essential. The focus of this work is thus the growth optimization of InGaN based heterostructures and a comparison of the growth conditions and the transport properties of $\mathrm{GaN} / \mathrm{lnGaN}$ and GaN/AlGaN structures.

The growth of high quality GaN/InGaN structures is challenging. The lower melting point of InN compared to GaN complicates the growth of InGaN and its application in $\mathrm{GaN}$ based heterostructures. Furthermore, InGaN has a tendency to segregate and the homogeneous and reproducible incorporation of indium is an issue. As for all III-N, the lack of suitable substrates results in the incorporation of dislocations. III-nitrides can not be pulled from the melt, as they decompose before melting unless very high pressures are applied. III-nitrides are therefore commonly grown on foreign substrates such as silicon carbide and sapphire. This results in the formation of dislocations because of the different thermal extension coefficients and the lattice mismatch between the substrate and the growing material. Dislocations act as strong scattering centers in electronic transport and mainly limit the electron mobility in optimized GaN/AlGaN structures.

The major developments in III-N growth were mainly achieved by metal organic vapor phase epitaxy (MOVPE) for optoelectronics, by hybride VPE (HVPE) for the growth of quasi-substrates and by molecular beam epitaxy (MBE) for high-electron mobility 2DEG heterostructures. For device production, VPE is most commonly applied. MBE is only used if high quality is necessary for device operation and the production rate are comparatively small, e.g. in the production of high frequency transistors and LASER diodes. This growth method is, however, a versatile technique which promotes great advances in research. It allows for in-situ characterization of growing films which permits a better understand of growth mechanisms.

GaN/AlGaN structures with the highest electron mobilities at low-temperature reported in the literature were grown by MBE. No high mobilities in GaN/InGaN structures grown by VPE techniques or MBE have been reported. MBE growth conditions that are applied for GaN/AlGaN structures might, however, result in high mobilities in GaN/InGaN heterostructures as well. Under typical MBE pressures III-N materials already decompose above $800{ }^{\circ} \mathrm{C}$ which is low compared to other growth techniques. High substrate temperatures above $1000^{\circ} \mathrm{C}$ are usually needed to produce smooth layers, which is essential for heterostructure growth. For GaN and AlGaN growth it is, however, well known and understood that metal-rich growth conditions result in smooth layers at temperatures far lower than applied for other growth techniques. This makes MBE suitable for InGaN growth, as this material requires the lowest growth temperatures of all ternary III-N due to decomposition. The impact of metal-rich growth conditions on the electron mobility in $\mathrm{GaN} / \mathrm{InGaN}$ structures is therefore studied in this work. 
The aim of this work is the MBE growth optimization of GaN/InGaN and GaN/AlGaN structures with regard to high electron mobilities at low temperatures. Studies of the low-temperature magnetoresistance identify the dominant scattering mechanisms for optimized structures.

Part one provides the background to experimental techniques. The MBE system and the mechanisms that determine the growth conditions for smooth nitride layers are presented. After a short introduction of reflection high energy electron diffraction (RHEED), the relevant applications of this in-situ method for the growth optimization process are explained in detail. The last chapter of part one focuses on the electrical characterization. Here, the implications of heterostructure parameters on carrier accumulation and the impact of scattering mechanisms on low-temperature transport are illustrated.

Part two presents the growth optimization in detail. Separate chapters address GaN, GaN/AlGaN and GaN/InGaN growth in order to determine the effects of key steps of growth on the structural quality and transport properties individually. A new method to estimate the maximum incorporable amount of indium by RHEED is presented for InGaN growth. The impact of the heterostructure design on the two dimensional carrier confinement is discussed for Ga/AlGaN and GaN/InGaN. The dominant scattering processes for electronic transport at low temperatures are determined by magnetoresistance measurements. A section gives the results for the application of GaN/AlGaN structures as $\mathrm{pH}$ sensors.

The thesis closes with a review of the achievements of this work comparing them to state of the art results from the literature. It states the limitations of MBE growth optimization of III-N and suggest future areas of work.

The appendix is mainly directed to people working in the lab. It gives some guidelines and recipes for grown structures. Furthermore, the use of RHEED in terms of image quality and data acquisition is described. The problem of temperature measurements in MBE is addressed and it is described how absolute temperature calibration was realized in this work. 
Part I

BACKGROUND OF EXPERIMENTAL METHODS 



\section{MOLECULAR BEAM EPITAXY OF III-NITRIDES}

Epitaxy is the growth of materials where the crystal structure and orientation of the growing film are determined by a host crystal. Molecular beam epitaxy (MBE) is an epitaxial growth technique which is well known for its precise thin film growth and in-situ monitoring during growth. It is a popular method for the production of compound semiconductor heterostructures, i.e. the stacking of layers of different materials. In this section, the background for MBE growth of III-nitride heterostructures is presented. Specific challenges that arise for the growth of smooth III-nitride layers by MBE compared to growth by other epitaxial techniques are discussed, and ways to meet them are presented. This section closes with a review on state of the art MBE growth conditions for high-quality GaN/AlGaN and GaN/InGaN heterostructure growth.

\subsection{GROWTH OF III-NITRIDES \\ 1.1.1 Historic development}

Group III-nitrides are used in a range of applications that hardly any other material system can cover. At the same time, the growth of these materials is particularly challenging. This is best reflected by the historic development of the growth of the material system.

GaN was first successfully synthesized in 1938 in the form of small crystallites [1], but it took until the early 70's before larger area layers were grown by hydride vapor phase epitaxy (HVPE) [2]. Back then, some basic concepts of today's III-nitride applications were already studied, such as LEDs and UV detectors [3]. However, poor crystal quality, a high unintentional n-type doping from point defects and a low reproducibility quickly reduced the excitement in those early years.

Higher quality material was grown in the mid 80's. The low-temperature deposition of AIN buffer layers on sapphire by Metalorganic vapor phase 
epitaxy (MOVPE) increased the quality of GaN (0001) significantly [4, 5]. Soon after, first heterostructures with AlGaN and InGaN were produced. A rapid development followed, which resulted in the first commercial devices by the mid 90's [6].

All the stated advances in nitride growth were achieved by vapor phase growth techniques and none by MBE. This technique was developed in the late 60's at the Bell laboratories [7]. It was successfully applied for the growth of other III-V compound semiconductors such as III-arsenides [8]. The development of GaN growth by MBE was, however, slow compared to other techniques because of serious challenges that were not overcome until the beginning of this century. Nowadays, some of the best nitride heterostructures are produced by MBE. The reason for the delayed success lies in the growth technique itself, as will be presented in the following sections.

\subsubsection{MBE setup}

The basic setup of an MBE system is illustrated in Figure 1.1. The idea is that separate particle beams supply the pure reactants to a heated substrate where they are adsorbed and incorporated. Effusion cells provide the metals. Here, the materials are placed in ceramic crucibles that are heated by a filament. A material flux is generated at sufficiently high cell temperatures, which depends on the crucible geometry, the cell temperature and the equilibrium vapor pressure of the source material. The cells can be shut mechanically to turn the beams on and off. For pressures below $10^{-5}$ mbar, the mean free path of atoms evaporated from the cells is larger than the distance between the source and the substrate, which is typically between $30 \mathrm{~cm}$ and $50 \mathrm{~cm}$. The materials thus form beams under low pressures. Consequently, MBE needs vacuum conditions and growth takes place in a growth chamber with reduced pressure.

Reactive nitrogen cannot be supplied by thermal evaporation. Pure nitrogen exists only as $\mathrm{N}_{2}$ and is one of the least reactive gases. In order to be used for growth, the molecules need to be excited or broken up. This is not possible by thermal excitation because of the high $\mathrm{N}-\mathrm{N}$ bond strength of $9.8 \mathrm{eV}$ [9]. Reactive nitrogen is thus produced by the dissociation of nitrogen molecules under plasma conditions.

Plasma sources consist of a crucible to which pure molecular nitrogen is supplied from a gas bottle. A mass flow controller regulates the gas flow. A perforated plate partially separates the crucible from the vacuum of the growth chamber. Pressures of 10-100 mbar are generated in this finite volume, which is excited by a high frequency field at a fixed frequency with variable power. Under specific power and nitrogen flow conditions, a plasma is generated which produces different types of reactive nitrogen. The ratio of activated nitrogen to supplied molecular nitrogen is of the order of $10 \%$ [9]. 


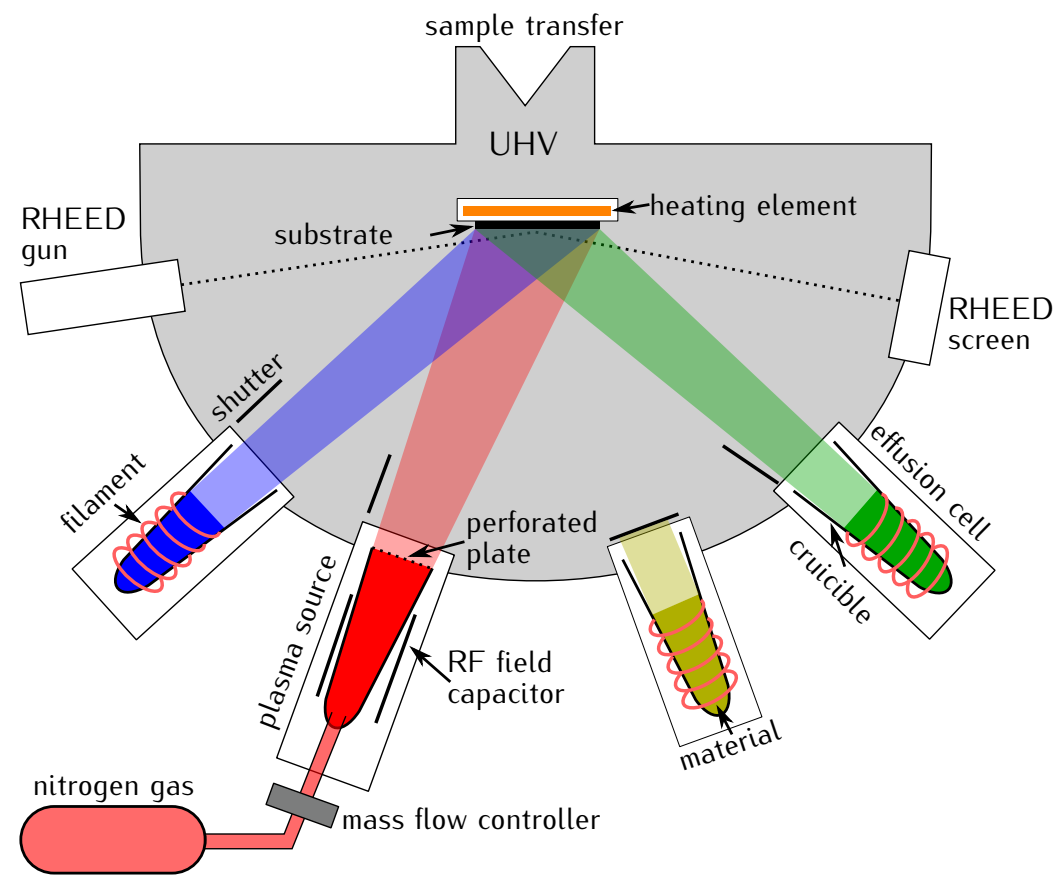

Figure 1.1: Schematic of an MBE growth chamber. Molecular beams form under vacuum conditions and mix in front of a heated substrate. Main components: Effusion cells: metals in crucible are heated by filaments. Plasma source: RF-capacitor excites finite volume which is separated from the vacuum by a perforated plate and gaseous nitrogen flow is controlled by a mass flow controller. RHEED: Electron gun generates electron beam which inclines under small angle onto the substrate and is detected at a phosphorus screen.

The optical spectrum of a generated plasma is shown in Figure 1.2. Several transitions can be observed which represent different nitrogen species. They can roughly be divided into atomic nitrogen and several different excited nitrogen molecules. Both types of nitrogen can contribute to growth which

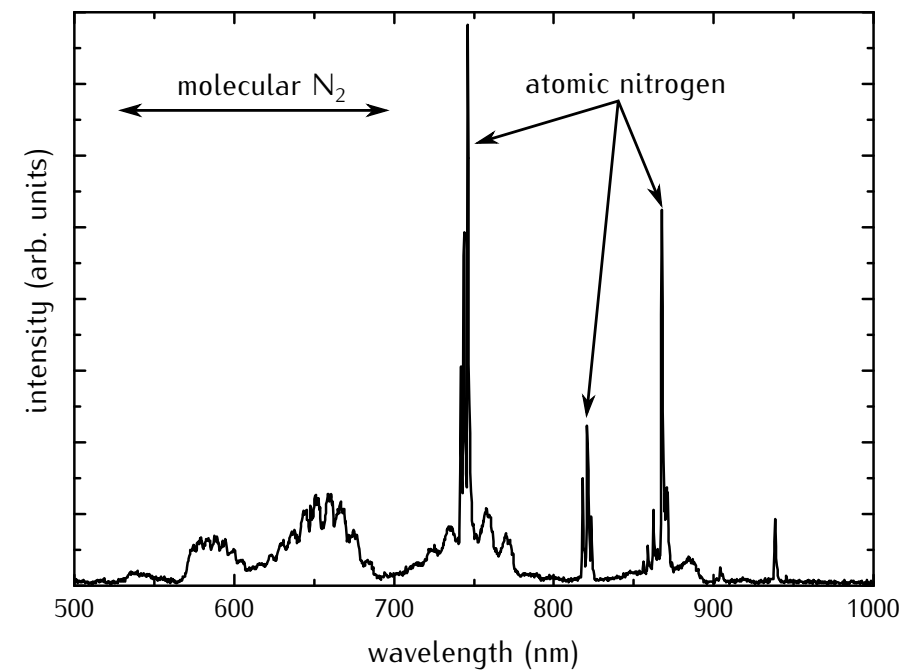

Figure 1.2

Optical spectrum of nitrogen plasma at $0.5 \mathrm{sccm} \mathrm{N}_{2}$ flow at a power of $300 \mathrm{~W}$. Atomic nitrogen emission at $745 \mathrm{~nm}, 821 \mathrm{~nm}$ and $868 \mathrm{~nm}$. Molecular nitrogen emission below $700 \mathrm{~nm}$ [10]. 
will be addressed in Section 1.2.

In $\mathrm{MBE}$, the arrival rate of atoms supplied from the cells and the desorption of atoms from the surface determine the growth rate. Technically achievable effusion cell temperatures in case of metals and feasible stable plasma conditions in case of nitrogen limit the deposition rate. For typical MBE growth conditions, the growth rates range from $1 \mu \mathrm{m} / \mathrm{h}$ down to 1 monolayer per several seconds. Such low growth rates result in sufficiently high surface migration of adsorbed atoms and allow for epitaxial ordering on appropriate substrates. However, the rate of contamination from the ambient must be small compared to the growth rate in order to produce clean crystals. For typical $M B E$ growth rates, this requires a base pressure below $p_{\text {base }}=10^{-10} \mathrm{mbar}$ which is the main reason for the technical complexity of $\mathrm{MBE}$ machines.

The commercial Veeco GEN II MBE system used in this work is shown in Figure 1.3. The ultra high vacuum (UHV) in the growth chamber needs to be maintained even if several samples per day are introduced into the system. This is possible with a three chamber setup which does not break the UHV at any time during regular operation ${ }^{1}$. The substrates are introduced via a load lock chamber with pressures below $p_{\text {base }}=10^{-7}$ mbar. They are then heated to reduce contaminations and water, condensated from the atmosphere. Afterwards, the substrates are transferred to a buffer chamber

1 growth chamber opened 2-4 times a year for maintenance

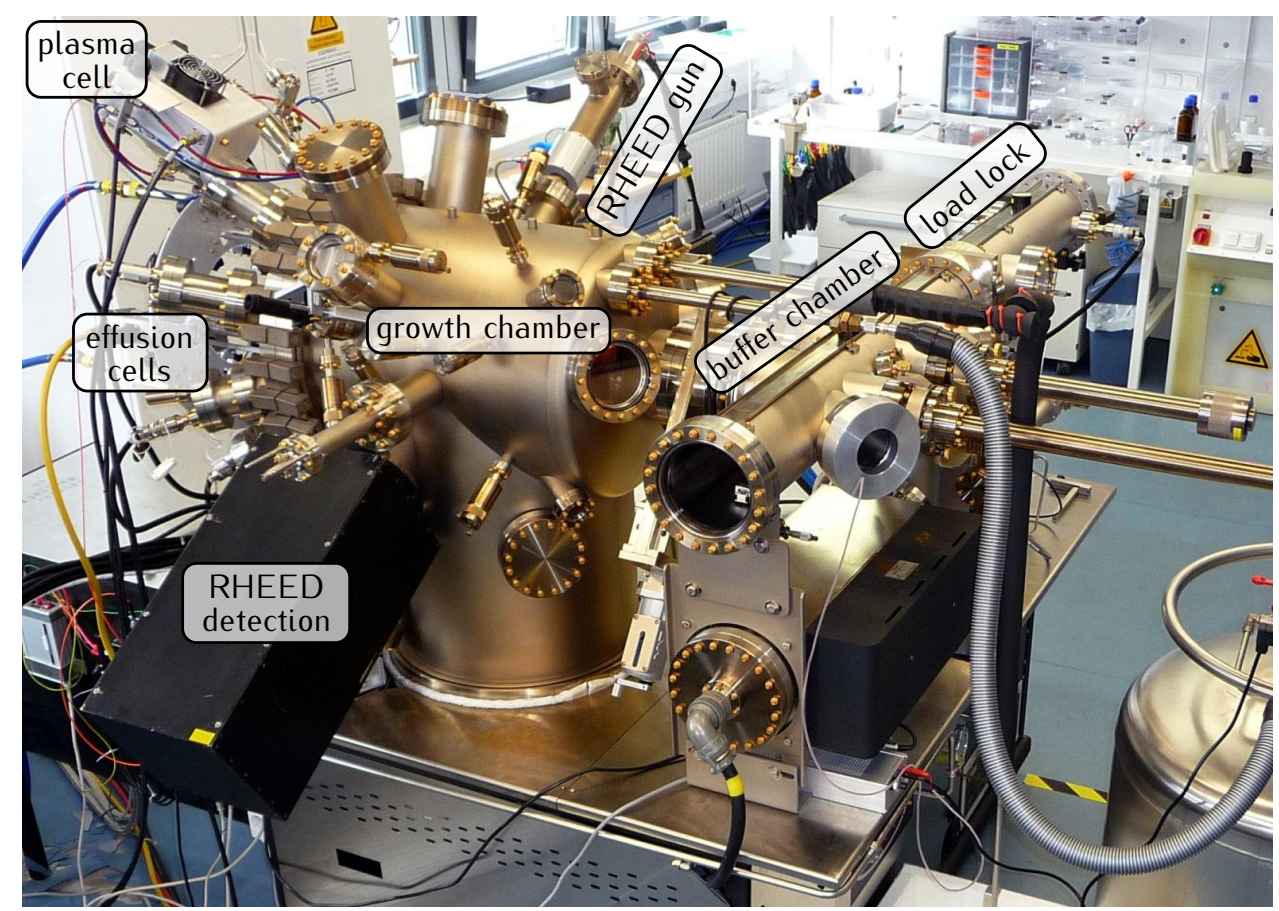

Figure 1.3: Veeco GEN II MBE used in this work. Substrates are introduced into the load lock with p pase $<10^{-7}$ mbar and transfered into the buffer chamber with $p_{\text {base }}<10^{-9}$ mbar before entering the growth chamber with $p_{\text {base }}<10^{-10}$ mbar. 
with $p_{\text {base }}<10^{-9}$ mbar and heated once more before transferred into the growth chamber with a base pressure below $p_{\text {base }}=10^{-10} \mathrm{mbar}$.

During growth, the pressure rises to $p_{\text {growth }}=10^{-5} \mathrm{mbar}$ because nitrogen is supplied to the plasma source. This does not conflict with MBE requirements. The higher pressure results from highly pure nitrogen and is no source of contamination. Furthermore, the pressure is still low enough to allow for the formation of molecular beams.

\subsubsection{Advantages and challenges of III-N growth by MBE}

$\mathrm{MBE}$ is an excellent technique for the growth of compound semiconductor heterostructures in principle and is remarkably successful in producing high quality III-arsenide structures. MBE grown structures exhibit sharper interfaces and doping profiles compared to vapor phase epitaxy (VPE) techniques. In VPE, carrier gases supply the reactants that flow over a heated substrate in a chamber. In order to switch between different materials, the flowing gases need to be changed. Fast switching between different materials is therefore more difficult than in MBE, where the supply can be shut off mechanically almost instantaneously in the UHV environment. Together with a low growth rate, MBE allows for a precise deposition of heterostructures. In addition, a higher level of security is needed for VPE as some of the carrier gases are toxic.

Another advantage of MBE is that all growth parameters can be controlled independently. In MOVPE, nitrogen is generated thermally from the carrier gas at the growing surface. The nitrogen supply consequently scales with the substrate temperature and growth at low temperatures with a high nitrogen supply is not possible. Such conditions are for example desired for high quality $\ln N$ growth.

The UHV conditions in MBE also support in-situ monitoring of the growth process by mass spectrometry, ellipsometry or reflection high-energy electron diffraction (RHEED). This allows not only for a precise control of the growth but also provides insight into growth mechanisms (see Chapter 2). Vacuum conditions are thus the origin of the advantages of MBE growth compared to other techniques. Unfortunately, problems arise for the growth of III-nitrides for the exact same reason.

From the growth of III-arsenides by MBE and MOVPE, it is well known that the optimal growth temperatures for smooth films is about half the melting point of the growing material (see Section 1.3). For GaN with a melting point of $2500{ }^{\circ} \mathrm{C}$ [11], this implies growth at temperatures well above the decomposition temperature under MBE conditions as is shown in Figure 1.4. The decomposition rate exceeds typical growth rates already above $800{ }^{\circ} \mathrm{C}$. Higher growth temperatures can only be applied for higher nitrogen pressures. For MOVPE, where nitrogen is supplied by ammonia, the $\mathrm{NH}_{3}$ overpressure is typically in the range of bars. This allows GaN growth 


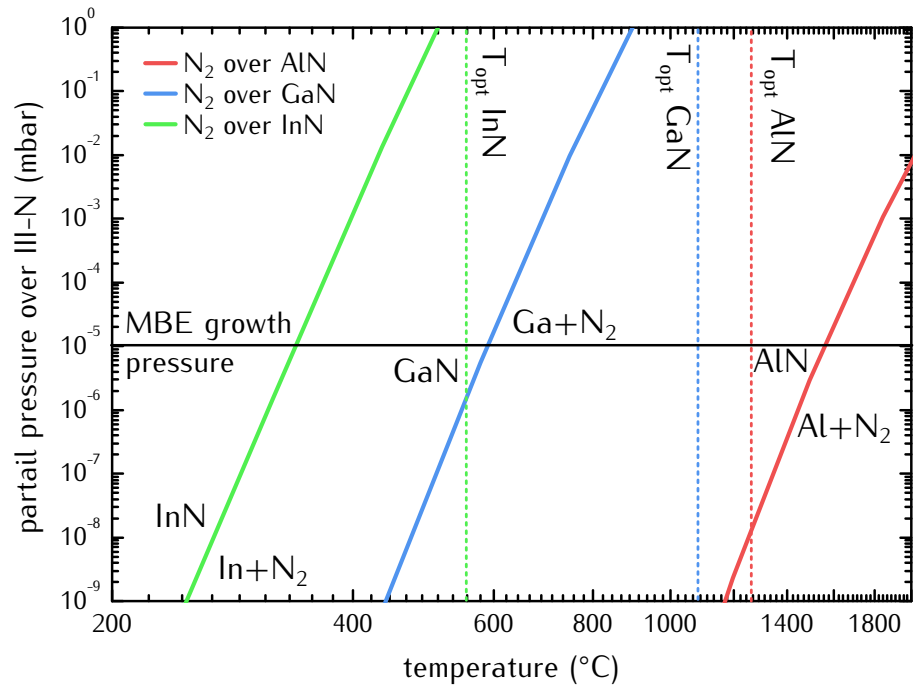

Figure 1.4: Equilibrium vapor pressure over substrate temperature for GaN, $\ln N$ and AIN [12]. The optimum growth temperature at half the melting point is indicated for the different materials. At typical MBE pressure of $\mathrm{p}=10^{-5}$ mbar only AIN can be grown at $T_{\text {opt }}$.

at temperatures above $1000^{\circ} \mathrm{C}$. For MBE growth, such high temperatures cannot be attained because lower pressures are essential for the generation of molecular beams.

First attempts to grow high quality GaN by MBE therefore focused on increasing the nitrogen pressure. This was achieved by ammonia-MBE where active nitrogen is supplied by $\mathrm{NH}_{3}$. High ammonia overpressures resulted in the successful growth of GaN up to substrate temperatures of $950{ }^{\circ} \mathrm{C}$ [13]. The results were promising, but growth by this technique has its drawbacks. One is that ammonia has corrosive effects on the metals of UHV components. Furthermore, a high level of hydrogen is incorporated into the growing film from the ambient. The growth by MBE with plasma sources is thus more commonly employed than ammonia MBE.

The game changer for MBE growth of III-nitrides was the discovery and explanation of high quality growth under gallium and indium rich conditions by adlayer enhanced diffusion (see Section 1.4). This effect allows droplet free growth and produces high quality GaN (0001) and AlGaN (0001) layers at temperatures below $800^{\circ} \mathrm{C}$.

Despite this big improvement, one problem still remains which results from the low growth rate in MBE. Nitrides lack of suitable substrates and are typically grown on foreign substrates such as sapphire (see Section 1.5.1). The grown layers are highly strained because of the large lattice mismatch. This strain reduces by the incorporation of dislocations at the substrate/GaN interface. The amount of dislocations can only be reduced if they annihilate or leave the growing crystal at its boundaries. For smooth GaN (0001) layer growth, the dislocations follow the growth direction $[14,15]$ and a reduction 
of the dislocation density during growth is small. Growth of rough 3D structures at lower substrate temperatures is thus a common approach in almost all growth techniques to reduce the dislocation density. Here, dislocations can annihilate because they do not advance parallel. The 3D structures are then overgrown by thick, smooth GaN layers. The resulting quality increases with the thickness. State of the art layers grown by MOVPE with thicknesses above $5 \mu \mathrm{m}$ result in dislocation densities of $10^{7} \mathrm{~cm}^{-2}$ [16]. HVPE grown structures with thicknesses of about $50 \mu \mathrm{m}$ exhibit even lower densities of $10^{6} \mathrm{~cm}^{-2}$ [17].

The MBE growth of layers with comparable thickness is time consuming. Furthermore, the dislocations are more mobile at the higher growth temperatures applied in other epitaxy techniques. Therefore, even the lowest reported dislocation densities in GaN layers grown by $\mathrm{MBE}$ on sapphire of $10^{8} \mathrm{~cm}^{-2}$ [18] are much higher than those produced by other techniques. State of the art MBE structures are thus commonly grown on commercial templates. These are III-nitride layers with low dislocation densities grown by other techniques such as MOVPE and HVPE on sapphire or SiC. A rising number of publication reports on the MBE growth on bulk GaN with even lower dislocation densities of $10^{3} \mathrm{~cm}^{-2}$ [19]. These substrates have become available recently but are still expensive and therefore not widespread yet. With the metal-rich growth conditions and the use of high quality substrates, III-nitride growth by MBE finally matured. Today, the highest quality IIInitride heterostructures are grown by MBE. The technique is however not applied in industry. Here, mainly MOVPE is applied because it allows for higher production rates. MBE growth of III-nitrides has its main implications in research.

\subsection{SURFACE PROCESSES DURING MBE GROWTH}

Crystal growth always takes place at the surface. The interplay of processes on the surface therefore determines the MBE growth conditions and is presented in this section. An overview of the possible processes is depicted in Figure 1.5. Impinging atoms are adsorbed on the surface where they diffuse. After some time, they either nucleate, incorporate or desorb. Already incorporated atoms may also decompose at higher temperatures.

Growth is per definition a non equilibrium process. If a vapor and a solid are in equilibrium, there will be no effective mass transport. Although various approaches on a thermodynamic basis exist, the description of growth by kinetic processes was chosen in this work. The kinetics describe the way of a system into equilibrium. This description accounts for activation barriers and explains the reactions via rates. It is thus directly related to the growth conditions which themselves are characterized by rates. In the following, the different processes are examined considering the case of GaN growth. The physical adsorption of atoms on a surface depends on the arrival rate, in- 
Figure 1.5

Processes on the surface during growth with atoms impinging. Typical path: atoms are adsorbed, then diffuse and incorporate, nucleate or desorp. At higher temperatures decomposition takes place.

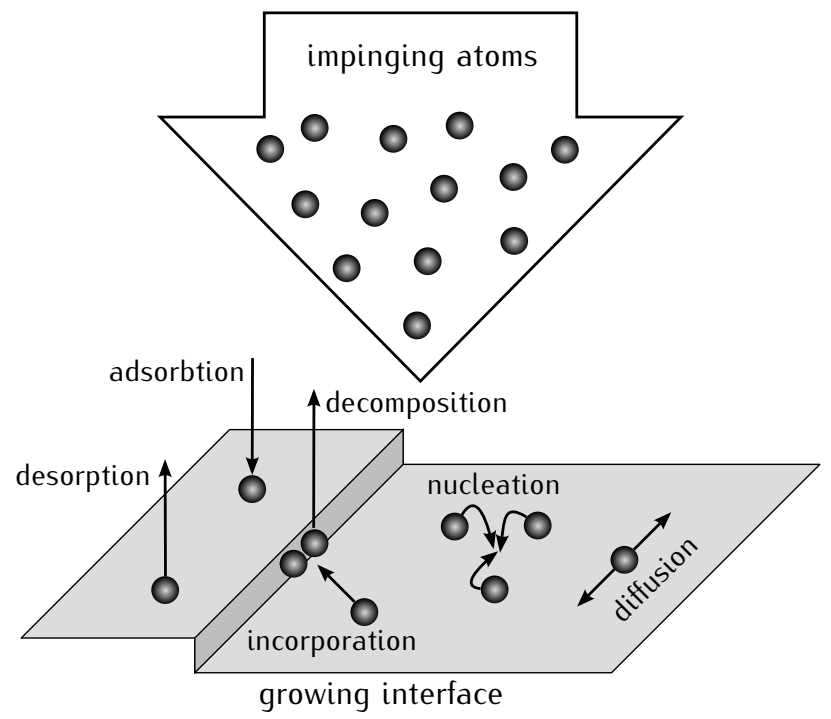

teraction between different elements, availability of possible adsorption sites and an activation barrier. The resulting adsorption rate can be characterized by the deposition rate of impinging atoms and a sticking coefficient which is the ratio of impinging atoms to atoms actually adsorbed. The adsorbed atoms are weakly bound to the surface [21]. For GaN growth, the deposition rate, which is given by the material flux from effusion cells, mainly determines the adsorption rate. The sticking coefficient is close to unity for gallium and nitrogen under regular GaN growth conditions [22] and can therefore be neglected.

Incorporation means the chemical bonding of the adsorbed atoms to the crystal surface. In the case of GaN growth, atomic Ga incorporates by bonding with a nitrogen atom. Under MBE conditions, this reaction is energetically not favored because liquid gallium and gaseous nitrogen have a lower potential energy compared to solid GaN as shown in Figure 1.6. The kinetic barrier of the reaction, however, can be overcome for the reaction of Ga with a variety of excited molecular and atomic nitrogen species. The kinetic barrier hinders the backward reaction of GaN to atomic gallium and molecular nitrogen in the ground state which allows GaN to grow.

Figure 1.6

activated nitrogen

GaN reaction

Comparison of the potential energy of activated nitrogen with Gibbs free energy for the reaction: $2 \mathrm{Ga}(\mathrm{l})+\mathrm{N} 2(\mathrm{~g}) \rightleftharpoons 2 \mathrm{GaN}(\mathrm{s})$ at typical growth conditions [20]. The kinetic barrier is overcome by activated nitrogen and hinders decomposition.

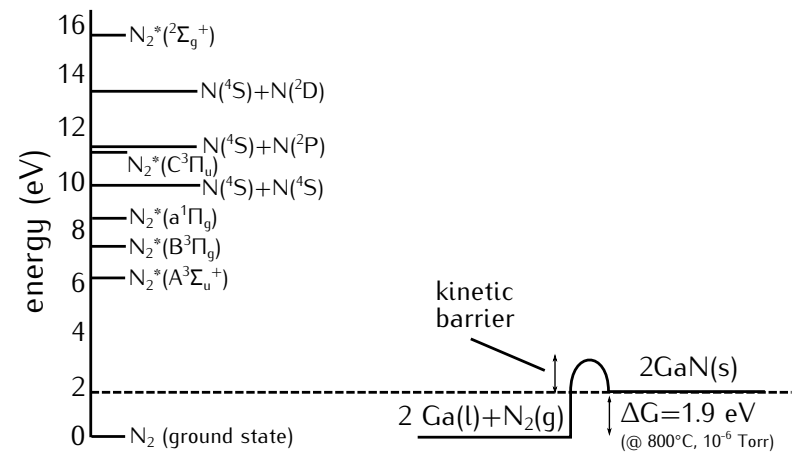




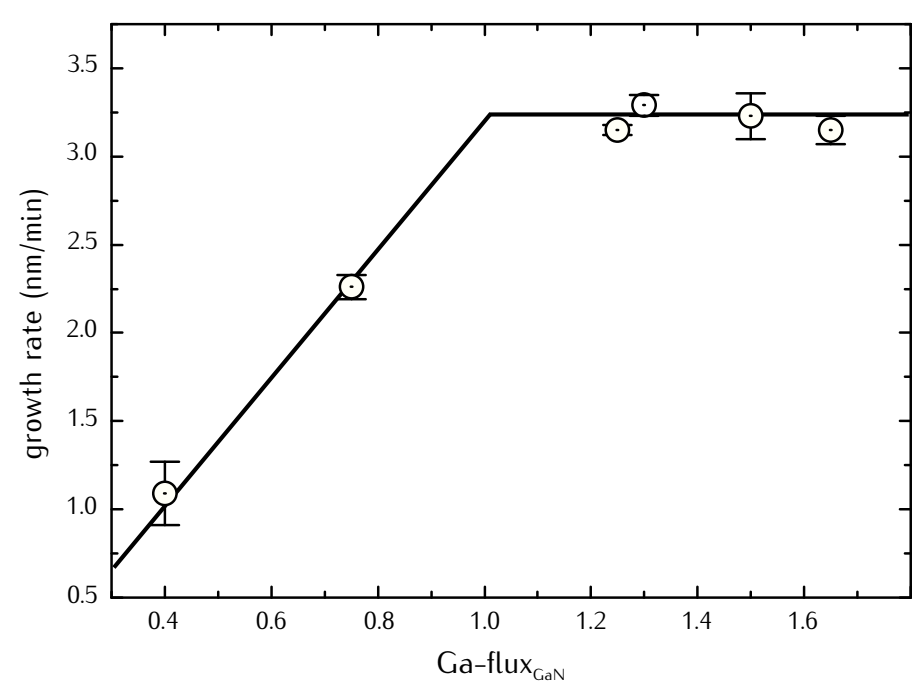

Figure 1.7: Growth rate of GaN depending on gallium flux under constant nitrogen flux. The Ga-flux is given normalized to the GaN stoichiometry. Three regimes are defined: $\mathrm{Ga} / \mathrm{N}<1: \mathrm{N}$-rich, $\mathrm{Ga} / \mathrm{N}=1$ : stoichiometric and Ga/N > 1: Ga-rich

The incorporation rate determines the growth rate. In Figure 1.7, the growth rate over Ga-flux for a constant $\mathrm{N}$-flux is shown. For low Ga-fluxes, the incorporation rate increases with the Ga-flux. This means that every additional gallium atom finds a nitrogen atom for incorporation. This range is called $\mathrm{N}$-rich because there is an oversupply of nitrogen and $\mathrm{Ga} / \mathrm{N}<1$. At high Ga-fluxes, the growth rate is constant because all available nitrogen atoms are already incorporated. This region is called Ga-rich because Ga/N $>1$. Such diagrams give the surface stoichiometry at Ga/ $\mathrm{N}=1$.

Desorption is the process of atoms leaving the surface through thermal energy gain. Its rate exponentially increases with the surface temperature. The kinetic barrier for this process depends on the bond strength between the adsorbate and the substrate. In addition, it depends on the desorption path, which is different for Ga and $\mathrm{N}$. The desorption rate over temperature for $\mathrm{Ga}$ and $\mathrm{N}$ from a GaN surface is given in Figure 1.8 [23]. The activation energy for Ga desorption is much lower than for $N$. The reason is that nitrogen can only desorb as ground state $N_{2}$ because other states would require energies higher than those supplied thermally by the substrate. One possible desorption path for $\mathrm{N}$ is the bonding of two nitrogen atoms diffusing on the surface. The desorption process of nitrogen is therefore related to surface diffusion. This is different for gallium atoms which can desorb directly. Another possibility for nitrogen desorption is the bonding of an adsorbed with an incorporated nitrogen atom. The probability of this reaction increases with temperature and represents a decomposition process. A high amount of atomic nitrogen in the impinging nitrogen flux therefore increases the decomposition rate of $\mathrm{GaN}$ at higher temperatures [25]. 
The desorption rates of $\mathrm{Ga}$ and $\mathrm{N}$ below $700^{\circ} \mathrm{C}$ are lower than the typical incorporation rates. The desorption rate of Ga becomes greater than incorporation rate applied in this work at about $700{ }^{\circ} \mathrm{C}$. Above this temperature, the Ga-flux needs to be increased in order to maintain stoichiometric growth conditions. The desorption rate of $\mathrm{N}$ exceeds the typical incorporation rate at temperatures above $750{ }^{\circ} \mathrm{C}$. For fixed nitrogen conditions, this leads to a decreased growth rate. At even higher temperatures, the nitrogen desorption rate exceeds that of gallium.

GaN is never thermodynamically stable under MBE growth conditions with nitrogen pressures around $10^{-5}$ mbar. The kinetic barrier of decomposition is, however, high and the decomposition rate exceeds the growth rate only at higher temperatures as shown in Figure 1.8. For temperatures above $800{ }^{\circ} \mathrm{C}$, the decomposition rate will exceed the incorporation rate applied in this work and the crystal will be etched rather than grown.

All observable growth modes producing smooth layers, wires or quantum dots arise from the interplay of the described processes mainly because of their impact on surface diffusion. While longer diffusion length of adatoms result in smooth layers (see Section 1.3), short diffusion produces rough structures. This emphasizes the importance of surface diffusion.

The diffusion length is given by the diffusion coefficient and the time an atom stays on the surface. The diffusion coefficient increases exponentially with temperature. The residence time is governed by other surface processes.

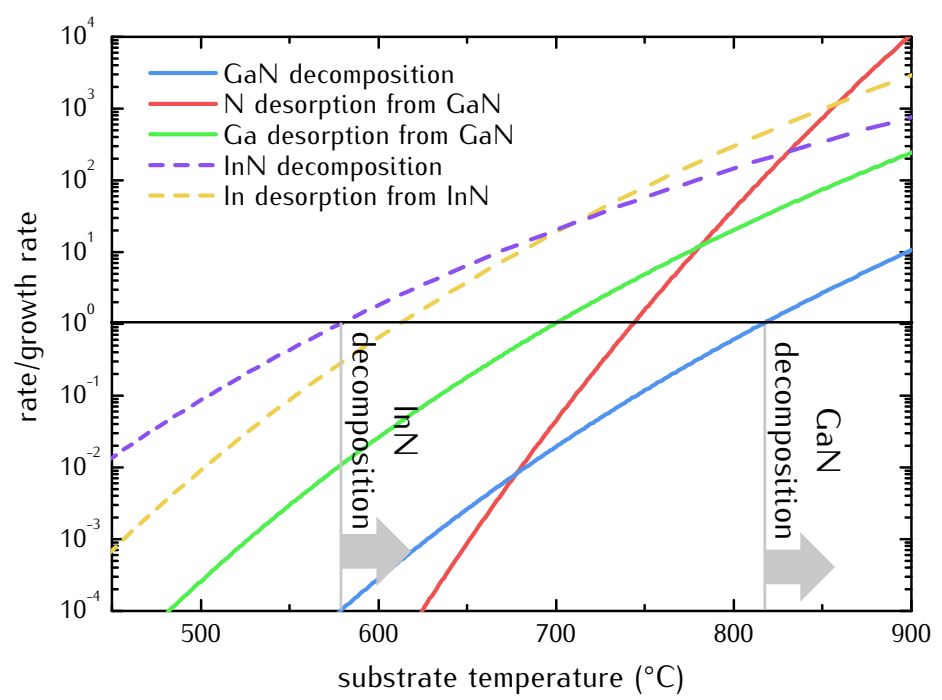

Figure 1.8: Rates of various surface processes over temperature for typical MBE $\mathrm{N}_{2}$ pressures given normalized to a growth rate of $3 \mathrm{~nm} / \mathrm{min}$ used in this work: $\mathrm{N}$ desorption from GaN (0001) surface, Ga desorption from GaN (0001) surface, GaN (0001) decomposition. Significant Ga desorption is achieved below the decomposition temperature at $800{ }^{\circ} \mathrm{C}$. For comparison: In desorption from $\ln N(0001)$ surface and $\ln N(0001)$ decomposition $[23,24]$. 


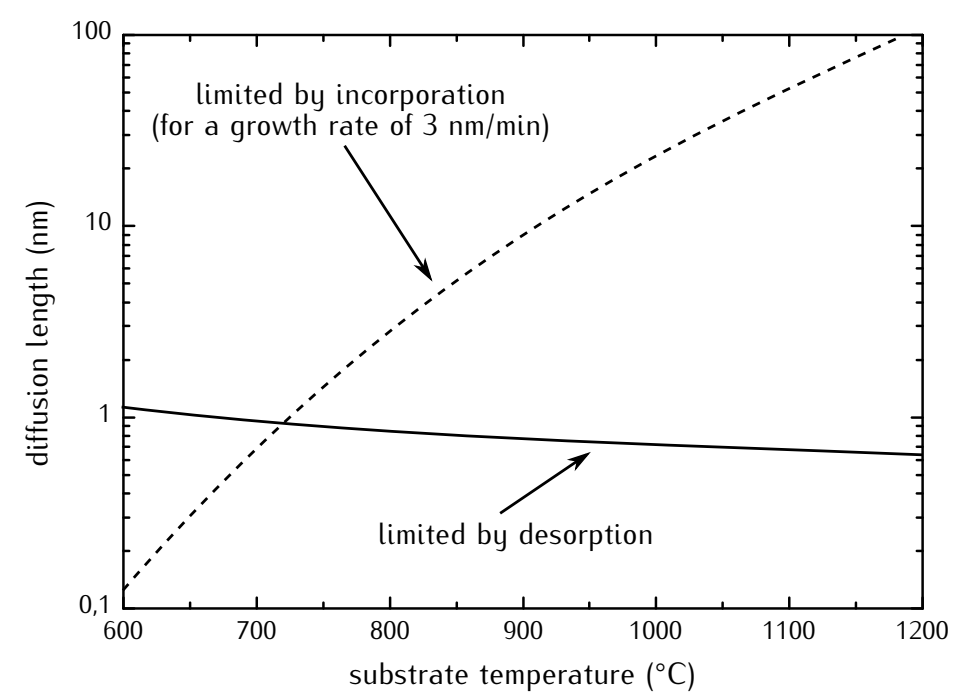

Figure 1.9: Diffusion length of gallium on GaN (0001) over temperature: Limited by incorporation for a growth rate of $3 \mathrm{~nm} / \mathrm{min}$ and by desorption [23]. A Maximum diffusion length for $\mathrm{Ga}$ is achieved around $700^{\circ} \mathrm{C}$.

An atom arriving on the surface might stay there until it either desorbs or incorporates. The desorption rate thus limits the diffusion length at higher temperatures. If the growth rate is increased, the time till incorporation shortens and rougher surfaces are the result. The diffusion length in MBE therefore does not simply increase with temperature and is limited by the incorporation rate at low temperatures and by the desorption rate of the materials at higher temperatures. An example of the diffusion length of $\mathrm{Ga}$ is given in Figure 1.9. The longest diffusion length is expected slightly above $700^{\circ} \mathrm{C}$.

The $\mathrm{Ga} / \mathrm{N}$ ratio also influences surface diffusion. In principal gallium is more mobile than nitrogen. For $\mathrm{N}$-rich conditions, the arriving Ga atoms are almost directly incorporated resulting in a short diffusion length. For Ga-rich conditions, the surfaces are smoother which is mainly the result of an enhanced nitrogen diffusion under a stable Ga-bilayer which will be discussed in Section 1.4.

\subsection{TWO DIMENSIONAL GROWTH MODES}

A two dimensional growth is essential for smooth interfaces in heterostructures. Before engaging the optimization of growth parameters, this section presents a brief physical treatment of the two dimensional growth of crystals. Only one material is treated for better illustration, but the derived conclusions also apply for binary and ternary materials. The background to this chapter, including the derivation of the formulas is found in the literature $[26,27]$.

A substrate is needed for epitaxial growth from a vapor phase. Further growth 
Figure 1.10

Cubic model crystal with 5 different possible incorporation sites: 1 and 2 are very rare, 4 and 5 increase the surface energy, 3 mainly contributes to growth

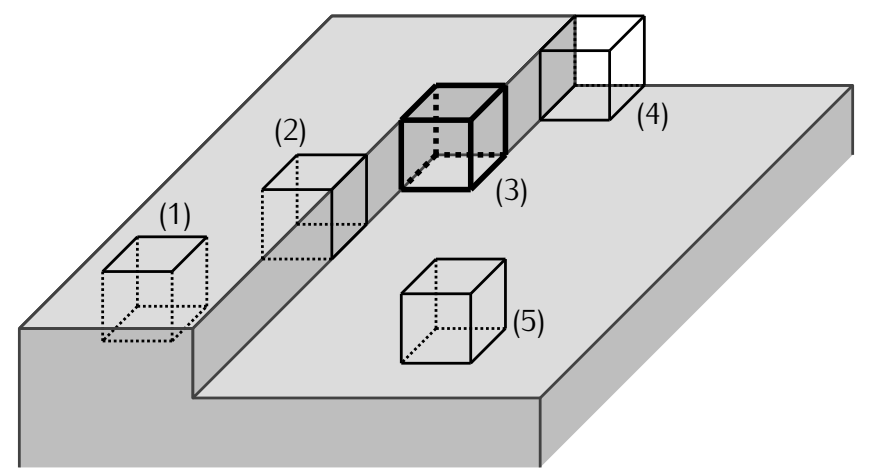

of a layer on the substrate occurs if a driving force induces a growth rate $R$ larger than zero. The growth rate is the difference between the atoms arriving at the surface and those leaving through desorption. The number of arriving atoms per time and area $R_{a b s}$ is expressed by a function of the ambient vapor pressure $p$ :

$$
R_{a b s}=\frac{p}{\sqrt{2 \pi m k_{B} T}}
$$

here $m$ is the mass of an atom, $k_{B}$ the Boltzmann constant and $T$ the temperature.

No growth occurs in equilibrium, and the number of atoms arriving at the surface is equal to the ones leaving. The desorption rate $R_{d e s}$ is therefore equal to the arrival rate of atoms under the equilibrium vapor pressure $p_{e}$ :

$$
R_{d e s}=\frac{p_{e}}{\sqrt{2 \pi m k_{B} T}}
$$

As mentioned, the difference between these two rates is the growth rate. It is therefore proportional to the difference of the vapor pressure and the equilibrium vapor pressure:

$$
R_{\max }(T, \sigma)=R_{a b s}-R_{d e s}=A(T) \frac{p-p_{e}}{p_{e}}=A(T) \cdot \sigma
$$

with the supersaturation of the vapor phase $\sigma=\frac{p-p_{e}}{p_{e}}$ and a temperature depended factor $A(T)$. The driving force can thus be identified as $\sigma$.

The derived growth rate describes only a maximum limit because it implies that every atom that enters the surface is incorporated into the crystal. This is only true in a particular case which can be concluded by examining possible incorporation sites on a model cubic crystal as shown in Figure 1.10. A real crystal is never perfectly smooth. Therefore, the model crystal has one exemplary step with a kink site. This step may represent a step due to a miscut of the host crystal or a part of an island on the surface.

Five different sites of incorporation are identified which differ in terms of energy and availability. The possibility of incorporation depends on these two conditions. Incorporation on sites 1 and 2 is energetically favored because it 
reduces the surface energy. However, once those sites are occupied they are not available for further growth. In contrast, sites 4 and 5 are very common on the surface, but incorporation on those sites increases the surface energy. Incorporation at sites 1, 2, 4 and 5 thus contributes little to growth.

Incorporation on site 3 does not change the surface energy. In addition, this site moves along the step if occupied. This is why sites like number 3 mainly contribute to growth. This site is called half crystal position because exactly half of its surface is attached to the crystal. Consequently, the detachment of an atom at this site requires the same energy as is gained by detachment, i.e. every atom entering the site with a certain probability is just as likely to leave. The maximum growth rate was derived for this condition. Therefore, a crystal that consists only of half crystal positions grows with the highest growth rate.

A crystal consisting only of the kink sites exhibits a maximum roughness. Almost no growth is expected for a perfectly smooth surface in this picture. A more realistic stepped surface with terraces of the length $\lambda$ is therefore investigated (Figure 1.11).

The way atoms incorporate at a step depends on the temperature. For GaAs growth, it has been observed that the optimal growth temperature promoting two dimensional growth is about half the melting point of the material [28]. This can be related to the half crystal position site. At about half the melting temperature, the kink sites become active. This means that they can be detached at rates as high as the deposition rate. This leads to a growth of smooth edges instead of a zig-zack structure as illustrated in Figure 1.11. Adsorbed atoms remain on the surface only for a certain time before they

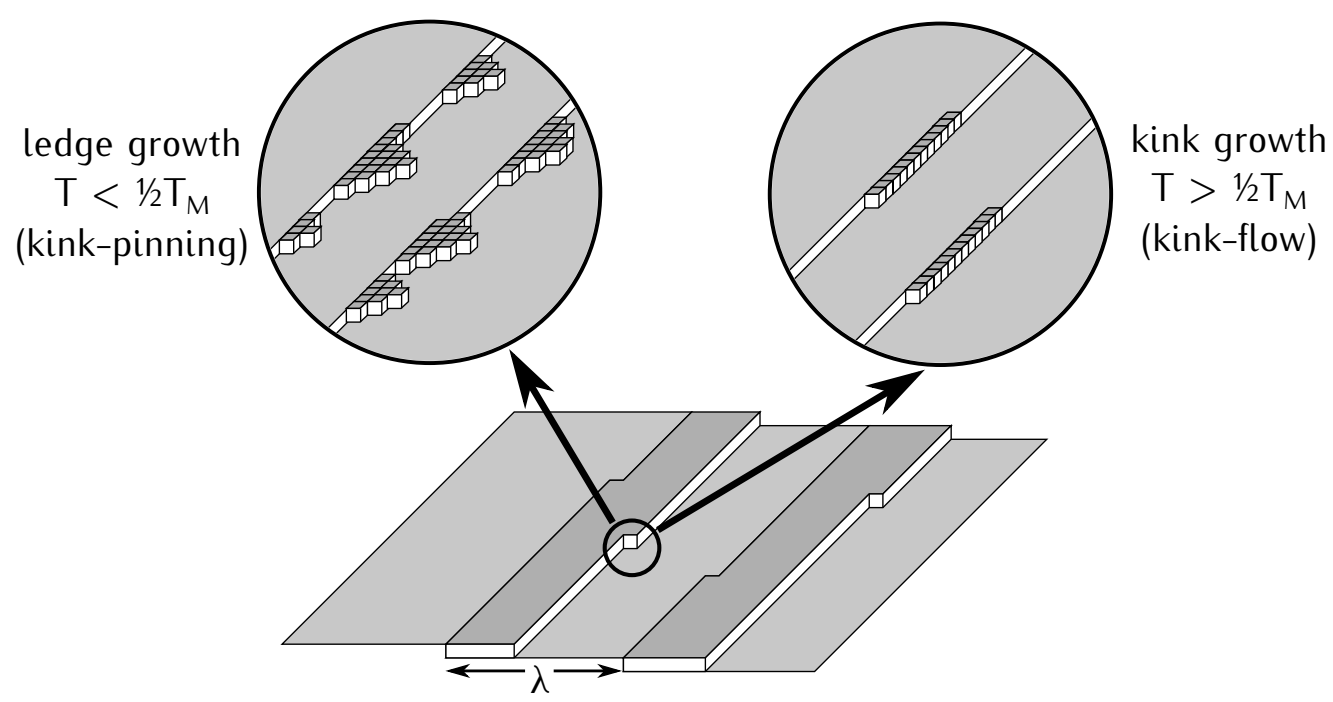

Figure 1.11: Comparison of ledge and kink growth expected for high and low temperatures [28]. Optimum growth temperatures are obtained above half the melting point of the growing material because kink sites become active. 
either incorporate into the crystal or desorb due to thermal fluctuations. During this time, they diffuse on the surface over a certain length $\lambda_{s}$. Incorporation at the step is only possible for atoms that can diffuse from the terraces to the step. For large spacings between the steps or small diffusion length the incorporation rate is reduced. The growth rate by step growth therefore depends on $\lambda_{s}$ and $\lambda$ :

$$
R_{\text {step }}=B\left(\frac{2 \lambda_{s}}{\lambda}\right) \cdot R_{\max }(T, \sigma)
$$

with the factor $B\left(\frac{2 \lambda_{s}}{\lambda}\right)<1$.

Nucleation has to be considered if $2 \lambda_{s}<\lambda$. Because atoms are preferably incorporated at a step, less free atoms are found in the vicinity of a step which can be expressed as a decreased $\sigma$. The supersaturation is thus maximal in the middle of the terrace and depends on $\lambda$ and $\lambda_{s}$ (Figure 1.12 a)).

Free atoms can attach to each other and form crystalline nuclei. By doing so, they gain energy if the crystalline phase is lower in energy. However, it costs surface energy. This results in a critical nuclei radius below which the nuclei decompose. The critical radius is related to a critical supersaturation $\sigma_{c}$ above which the nuclei will grow further. Nuclei grow on terraces if the supersaturation on the terrace of a stepped surface exceeds $\sigma_{c}$ (Figure 1.12 b)). This produces new steps and the nuclei grow two dimensionally. Since $\sigma$ depends on the diffusions length and the terrace width, so does the growth rate by nucleation $R_{\text {nuc }}$. The growth rate for nucleation is higher than for stepflow growth because nuclei generate their own steps.

For high supersaturations, the growth by nucleation causes rough surfaces due to kinetic roughening (Figure $1.12 \mathrm{c}$ )). The distance between the steps produced by the nuclei, increases during the lateral 2D nucleation growth. For high supersaturations, this leads to the formation of new nuclei on top of still growing nuclei. Therefore, the surface roughens with growth time.
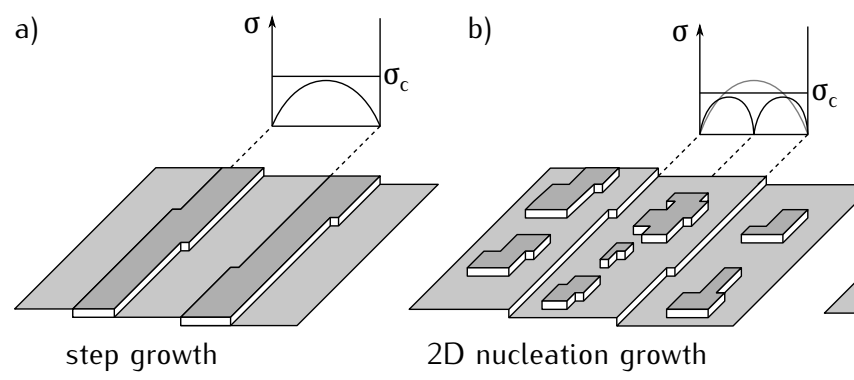

c)

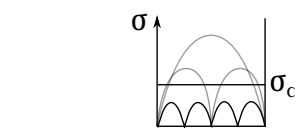

Ducleation growt

kinetic roughening

Figure 1.12: Comparison of 2D growth modes on a stepped surface depending on the supersaturation: a) $\sigma<\sigma_{c}$ - step growth: atoms incorporate at steps, b) $\sigma>\sigma_{c}-2 \mathrm{D}$ nucleation growth: growth also by nucleation between steps, c) $\sigma \gg \sigma_{c}$ - kinetic roughening: nucleation on growing nuclei roughens the surface over time. 


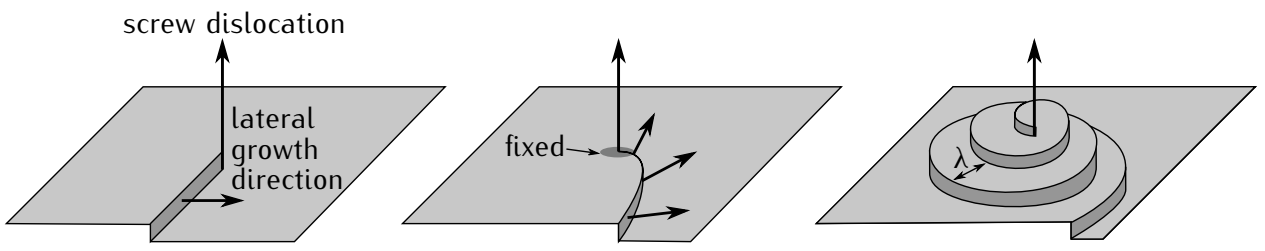

Figure 1.13: Schematic of spiral growth mode. A threading dislocation generates a step which is pinned at the intersection. By lateral growth a spiral with terraces of width $\lambda$ forms around this positions. The curvature of the spiral depends on the supersaturation $\sigma$.

Truly two dimensional growth by nucleation is only possible in a small range of supersaturations, and a roughening of the surface is very likely in this growth mode. Step growth on the contrary is always 2D and is thus preferred for heterostructure growth.

Another possible origin of steps are screw dislocations penetrating the surface (Figure 1.13). Screw dislocations are displacements of a fraction of a crystal plane. On the surface, this results in a step which is pinned at the intersection of the dislocation. If this step advances during growth is has to bow which results in a spiral. Further lateral growth of the spiral increases its curvature $\rho$ which leads to an increased edge energy. This slows down the lateral growth which ultimately results in a finite curvature:

$$
\rho_{c}=\frac{\gamma a}{k_{B} T \ln (1+\sigma)}
$$

with step energy per molecule $\gamma$, monolayer height $a$. Apart from the material parameters the geometry of the spiral depends only the supersaturation. The terrace width $\lambda$ formed by the spiral depends on the curvature and therefore on the supersaturation. For regular step growth, it was already shown that the growth rate depends on $\lambda$. Therefore, the growth rate for spiral growth is a supersaturation dependent modification of the maximum growth rate:

$$
R_{\text {step }}=C\left(\frac{2 \lambda_{s}}{\lambda(\sigma)}\right) \cdot R_{\max }(T, \sigma)
$$

with $C\left(\frac{2 \lambda_{s}}{\lambda(\sigma)}\right)<1$

It should be noted that spiral growth is a modification of the step growth. They are similar in quality. Step growth from miscut terraces and spiral growth always take place at the same time because both features are found on every real sample. An excellent example was observed during this work and is given in Figure 1.14.

The growth rates for the three different $2 \mathrm{D}$ growth modes are compared in Figure 1.15 for two different diffusion lengths. The morphology is determined by the mode with the highest growth rate at a given supersaturation. For 
Figure 1.14

Atomic force micrograph illustrating that spiral and step growth take place at the same time: three spirals in a stepped surrounding (SamplelD: G1020).

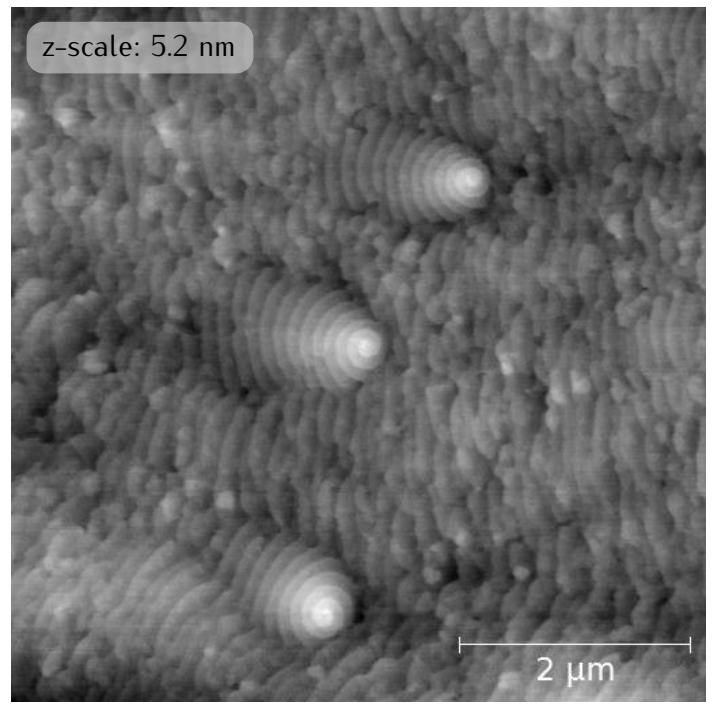

small supersaturation, the step growth is observed. For medium $\sigma$, the spiral growth produces higher growth rates. At higher $\sigma$, the 2D nucleation growth is observed. The growth rate by nucleation growth equals $R_{\max }$ at higher supersaturation. This illustrates the rough growth due to kinetic roughening, as $R_{\max }$ is only achieved on surfaces with maximum roughness. The transition between smooth and rough growth depends on the diffusion length. Smooth surfaces are only achieved for a high surface diffusion at high supersaturations.

Figure 1.15 illustrated, that different growth regimes are expected for different levels of supersaturation, i.e. how close to equilibrium the growth takes place. For real structures this can be observed by comparing a MOVPE to a MBE grown samples as done in Figure 1.16. Since step growth is only observed at very low levels of supersaturation, the growth by MOVPE takes
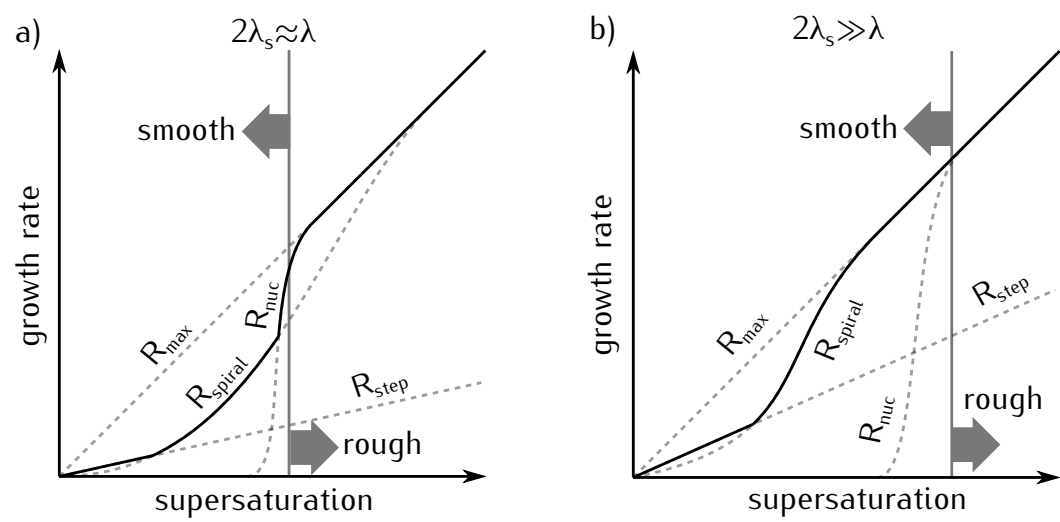

Figure 1.15: Growth rate over supersaturation for a) low diffusion length and b) high diffusion length, with the diffusion length $\lambda_{s}$ and a terrace width $\lambda$. The growth mode with the highest growth rate determines the morphology. Smooth growth is achieved by spiral and step growth, while nucleation growth results in rough surfaces due to kinetic roughening. 

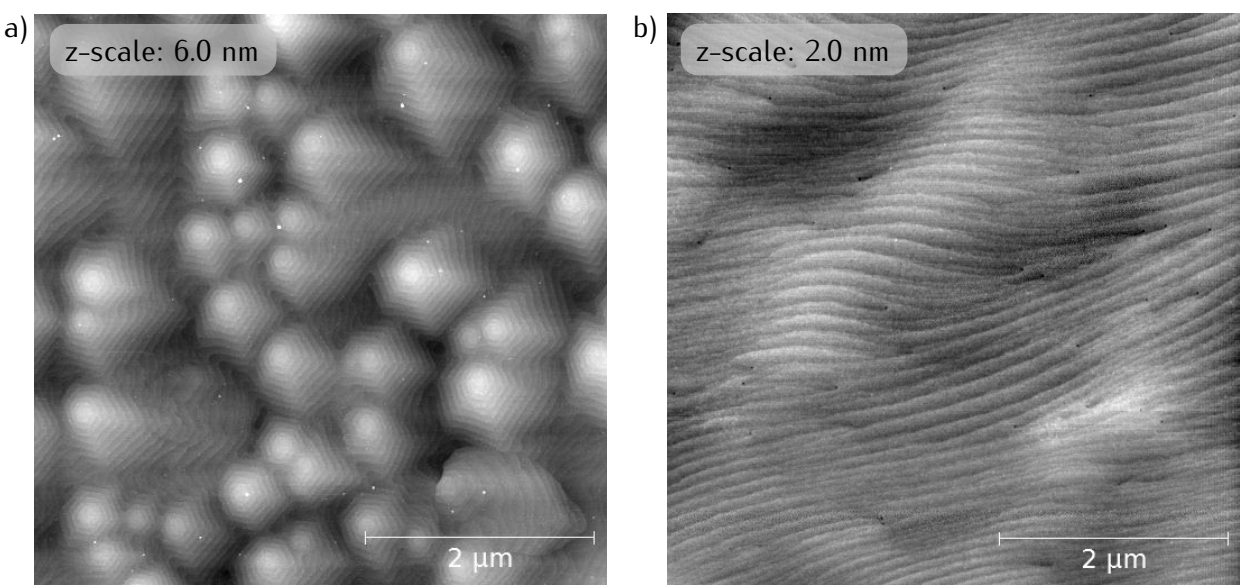

Figure 1.16: Atomic force micrograph of GaN (0001) grown by a) MBE and b) MOVPE. The spirals in MBE growth indicate that growth takes place further away from equilibrium than in MOVPE.

places closer to equilibrium than MBE growth. A more detailed discussion of the different morphologies for MBE and MOVPE grown structures is found in the literature [29]. In Figure 1.15 it also illustrated, that higher $\sigma$ require higher diffusion length for smooth growth. Therefore, large diffusion lengths are needed for the growth of smooth layers by MBE.

High diffusion lengths can be obtained at high growth temperatures. The activation of kink sites also suggests growth at high temperatures. As was mentioned in Section 1.1.3, this is a challenge for MBE growth compared to MOVPE because of decomposition under MBE conditions. It can however be faced by Ga-rich growth conditions as will be explained in the next section.

\subsection{GALLIUM AND INDIUM BILAYER}

The previous sections stressed the importance of a high growth temperature to activate kink sites and the importance of a high diffusion length for the MBE growth of smooth layers. For the MBE growth of III-nitrides, the decomposition limits the applicable temperature range (see Figure 1.4). Increased diffusion can thus only be achieved by a reduced kinetic barrier for surface diffusion. This section presents the positive effects of a gallium and indium bilayer coverage on the growing surface.

Early experimental findings revealed good crystal quality of MBE grown GaN (0001) for Ga-rich conditions [30]. Total energy calculations for different Ga coverages on a GaN (0001) surface later explained the experimental results [31]. The Ga atoms form a liquid bilayer on top of the GaN surface at growth temperature, which reduces the kinetic barrier for nitrogen diffusion. Under very Ga-rich conditions a laterally contracted bilayer of Ga atoms formed on the GaN (0001) surface, as shown in Figure 1.17, is energetically favored. The first layer orders according to the underlying Ga atoms in the 
Figure 1.17

Representation of the laterally contracted Ga-bilayer [31]. Ga layer 1 arranges according to Ga layer 2 of the GaN (0001) surface. Ga layer 0 is compressed and closer to the lattice constant of metallic gallium. Overall Ga content in layer 1 and 0 compared to layer 2: 2.33 .

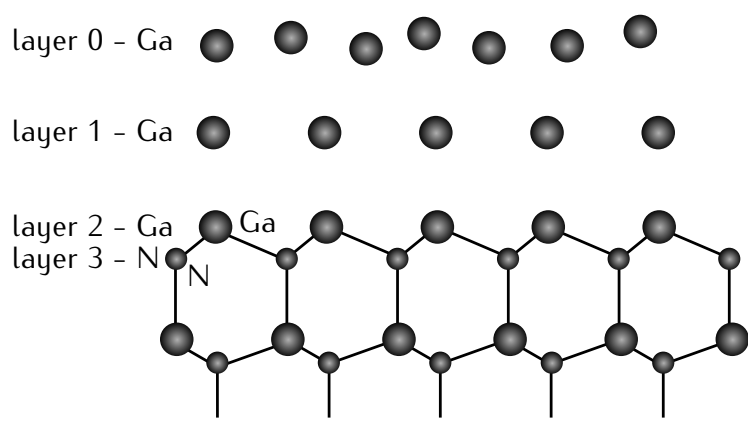

GaN lattice. The second layer on the contrary reduces the surface energy to a minimum by arranging closer to the lattice spacing of metallic gallium. This results in the arrangement of Ga atoms in a laterally contracted bilayer with a total coverage of $2.33 \mathrm{ML}$ of $\mathrm{Ga}$ with respect the GaN lattice. For indium on GaN (0001), a similar behavior was observed, with a coverage of 1.7 ML.

The bilayer model was experimentally investigated by monitoring the desorption time of Ga from the Ga-face GaN surface with RHEED [32]. The result is shown in Figure 1.18. A constant desorption time is observed for a wide range of employed Ga-fluxes. In the constant range, the surface coverage is $2.7 \mathrm{ML}$ of gallium. This supported the existence of a Ga bilayer which is stable for a certain range of deposition rates. Note that growth under these Ga bilayer stabilized conditions requires a sufficient Ga desorption as otherwise Ga accumulates in the form of droplets.

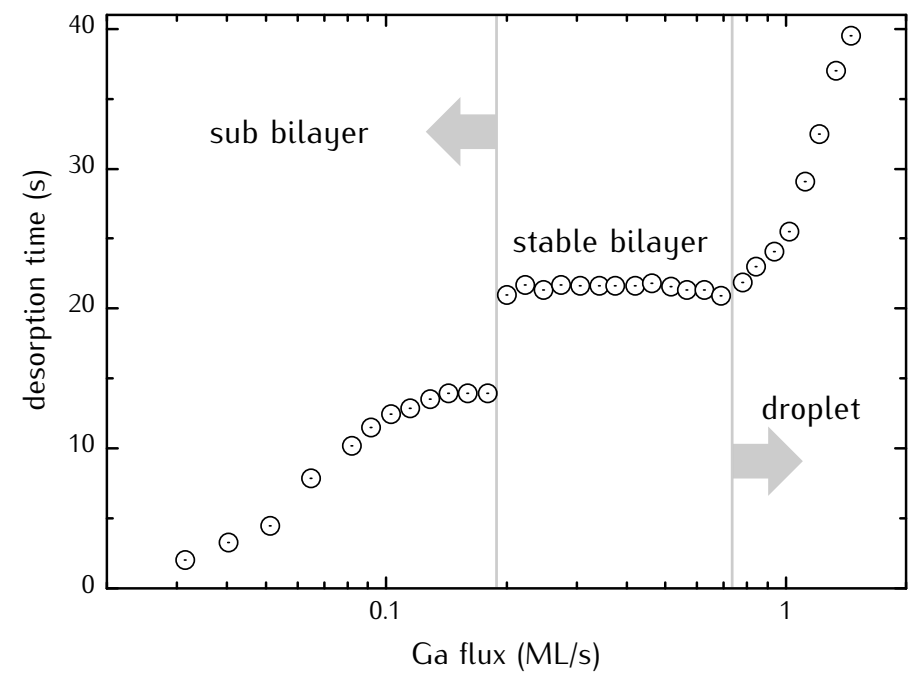

Figure 1.18: Ga desorption time over Ga-flux as measured by RHEED [32]. The constant desorption time at intermediate fluxes indicates the stable coverage of the Ga bilayer. The jump in the desorption time at $0.2 \mathrm{ML} / \mathrm{s}$ is explained by the change in the desorption characteristic as shown in Section 2.2.4. 
In order to explain the effects on the surface morphology, the surface diffusion of Ga and N for Ga bilayer covered GaN (0001) surface was investigated by density functional theory [33]. Results showed that both a Ga-bilayer and an In-bilayer significantly change the nitrogen diffusion. No energetic barrier was found for the diffusion of $\mathrm{N}$ through the metallic adlayer. In addition, the diffusion barrier for $\mathrm{N}$-atoms within the metallic layer is markedly reduced compared to the bare GaN (0001) surface from $1.5 \mathrm{eV}$ to $0.5 \mathrm{eV}$. This leads to a new lateral diffusion channel for nitrogen within the adlayer, resulting in a higher nitrogen diffusion length.

This alone cannot explain the observed spiral growth mode under bilayer conditions. An additional effect by the liquid metallic adlayer is the increased activation of kink sites. The presence of an adlayer is known to decrease the kinetic barrier for decomposition of GaN [34]. This implies, that already bound atoms can be detached from the crystal more easily. Therefore, the presence of a metal adlayer increases the activation of kink sites, which determines the optimum growth temperature for smooth growth (see Figure 1.11). It was reported that this process results in a kink activity at $750{ }^{\circ} \mathrm{C}$ for a excess $\mathrm{Ga}$ on $\mathrm{GaN}$ that is expected at $1050{ }^{\circ} \mathrm{C}$ for bare GaN surfaces [35]. The optimum growth temperature for $\mathrm{GaN}$ under metal rich conditions is thus lower than half the melting point.

The finding of the bilayer enhanced diffusion together with the lower optimum growth temperature were the breakthrough for MBE growth of III-nitrides. These two effects mainly affect the growth conditions for smooth III-nitride layers.

\subsection{MBE GROWTH CONDITIONS FOR III-NITRIDES}

The key aspects of III-nitride growth by MBE have been introduced in the previous section. In this section, the implications for the growth parameters, namely the substrate temperature and the material fluxes, for high quality III-nitrides growth by MBE are presented. Furthermore, growth conditions reported in the literature are given, which are the basis for the experimental part of this work.

It should be noted that the reproduction of results reported in literature is not straightforward. Many parameters depend on the machines used. The temperature of the substrate often deviates, as different calibration methods are employed. The plasma conditions also vary for different brands of plasma cells. In addition, the efficiency of the plasma cell reduces over time and growth conditions vary over time even for the same machine. Furthermore, the cleaning of the substrates often depends on parameters which can hardly be specified. Therefore, not only the optimization of growth conditions is essential for high quality growth but also the reproducibility. 


\subsubsection{Substrates}

Epitaxial growth is best performed homoepitaxially, which means growth on a substrate which is by all means identical to the growing film. The production of any bulk III-nitride crystal is however challenging. The synthetization is not possible from the melt because of the high melting temperatures and the weak III-N bond compared to $\mathrm{N}-\mathrm{N}$. Recently considerable progress was made by other techniques, such as ammonothermal growth, and bulk GaN wafers up to 2-inches are available [19]. However, such substrates are still expensive and growth on other substrates is more common.

The growth on foreign materials has produced reasonable results for a wide variety of substrates $[36,12]$. The most common by far are $6 \mathrm{H}$ silicon carbide $(6 \mathrm{H} \mathrm{SiC})$ and sapphire $\left(\mathrm{Al}_{2} \mathrm{O}_{3}\right)$. The lattice mismatch between $\mathrm{GaN}$ and $\mathrm{SiC}$ is about $4 \%$, which is much smaller than $16 \%$ for sapphire. This results in an increased crystal quality. However, $\mathrm{SiC}$ is expensive, making sapphire the substrate of choice in most cases.

The high lattice mismatch between GaN and sapphire results in a high density of dislocations. Their amount could be reduced over the last years down to $10^{8} \mathrm{~cm}^{-2}$ for GaN grown directly on sapphire by MBE [18]. The treatment of $\mathrm{Al}_{2} \mathrm{O}_{3}$ with activated nitrogen at low temperatures produces a thin AIN layer [37]. This layer is homogeneous and smooth and is the basis for thicker layers. The introduction of AIN or GaN buffer layers grown at low temperatures further increases the crystal quality. During this growth step, small islands form which then grow and coalesce [38]. The 3D growth allows for strain reduction and thus for a reduction of the dislocation density. The overgrowth of these 3D layers by thick, smooth layers results in a decent overall crystal quality.

Despite all these steps, III-nitrides grown on foreign substrates by MBE are of inferior quality compared to other techniques with higher growth rates. The substrate of choice for high quality III-nitrides are thus MOVPE or HVPE GaN templates with dislocation densities lower than $10^{8} \mathrm{~cm}^{-2}$.

\subsubsection{GaN}

The Ga/N ratio has a tremendous impact on the quality of GaN (0001) layers grown by $\mathrm{MBE}$ as shown in Figure 1.19. For $\mathrm{N}$-rich growth $(\mathrm{Ga} / \mathrm{N}<1)$, the layers are usually rough. The high amount of less mobile nitrogen on the surface decreases the diffusion length of Ga. It should however be noted that recently high quality layers were grown under $\mathrm{N}$-rich conditions for substrate temperatures close to the decomposition of GaN [39]. This approach is very promising but could not be reproduced in this work.

Under Ga-rich conditions, a sufficient Ga desorption rate has to be achieved to prevent the formation of droplets. This limits 2D growth to substrate temperatures higher than $670^{\circ} \mathrm{C}$. Different regimes can be identified above 


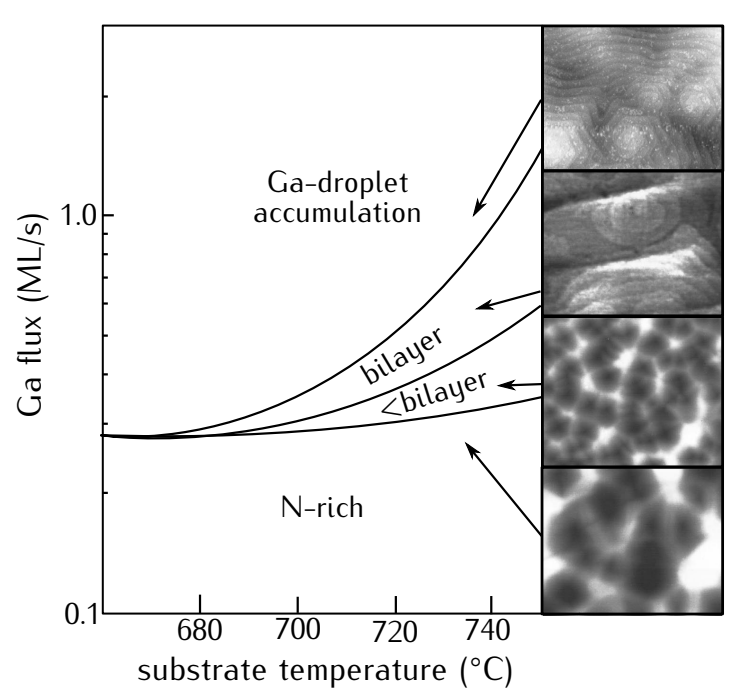

Figure 1.19

Growth regimes depending on Gaflux and substrate temperature as determined by RHEED desorption studies[42]. The best morphology is achieved slightly above the bilayer-droplet transition .

this temperature depending on the III/V ratio. Above the stoichiometry, the surface is covered by one ML of gallium or less. Nucleation growth is observed (see Section 2.2.3) which produces rough layers due to kinetic roughening. A further increase in the ratio leads to growth in the Ga bilayer stabilized regime. Smooth spiral growth is observed with roughnesses well below $1 \mathrm{~nm}$ [32]. At higher III/V ratios, the Ga desorption is not sufficient compared to the deposition rate and Ga droplets begin to form. The best results in terms of bulk electron mobility and morphology have been obtained for growth at the bilayer-droplet transition [40, 41].

Decomposition limits GaN growth at higher temperatures. Decomposition at $800{ }^{\circ} \mathrm{C}$ under typical MBE conditions was observed [25]. For Ga covered GaN surfaces, decomposition already starts at $720^{\circ} \mathrm{C}$ as the metallic adlayer reduces the kinetic barrier of decomposition [43]. Around this temperature, the highest Ga diffusion length is expected for typical growth rates of $3 \mathrm{~nm} / \mathrm{min}$ as was shown in Figure 1.9. High quality structures are therefore grown around $700{ }^{\circ} \mathrm{C}$ with a III/V ratio close to the bilayer/droplet transition.

The nitrogen conditions are given by the molecular nitrogen flow rate and the plasma power. It was reported that higher powers result in a higher density of point defects [44]. The reason is a damage of the growing film by high energy nitrogen species. A stable plasma at low powers is only achieved for low nitrogen fluxes. Conditions reported in the literature thus vary, with $\mathrm{N}_{2}$ flows from 0.2 to $1.0 \mathrm{sccm}$ at excitation powers below $300 \mathrm{~W}$ $[45,46]$.

\subsubsection{AlGaN}

The optimum growth conditions for ternary alloys are a combination of those for the involved binary compounds. The optimum growth conditions for GaN have been explained. Similar consideration apply to AIN growth as well. The decomposition temperature for AIN under MBE conditions is considerably 


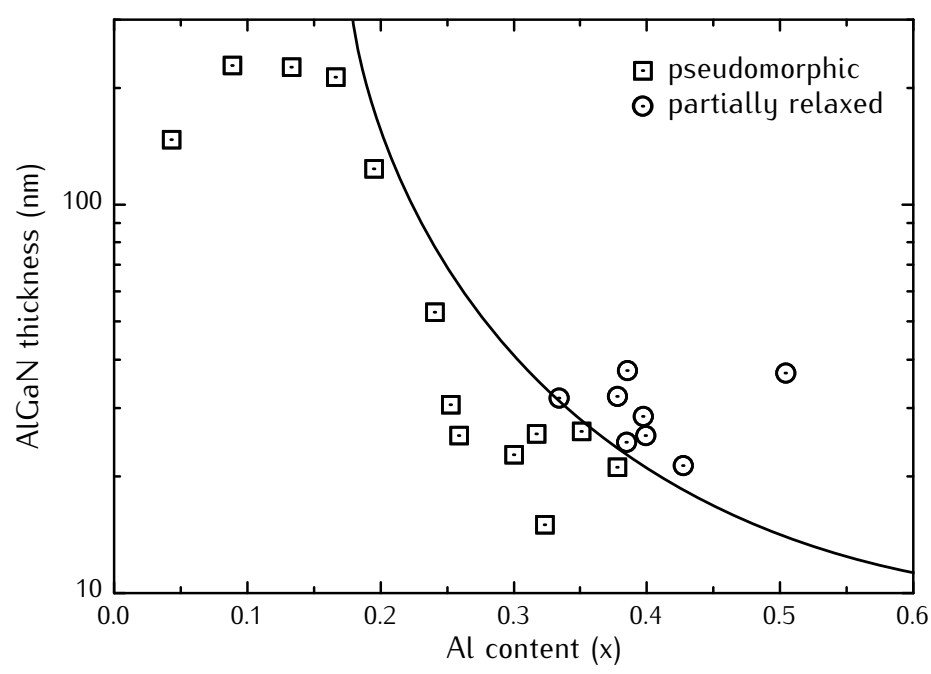

Figure 1.20: Critical thickness for AlGaN relaxation over Al concentration [47]. For heterostructure growth the AlGaN thickness is typically below $50 \mathrm{~nm}$. Here, no relaxation is expected for $x_{A l}<0.25$.

higher than that of $\mathrm{GaN}$ as seen in Figure 1.4. This would allow for growth at half the melting temperature. However, this results in the decomposition of other nitride layers that are part of a heterostructure and is thus not suitable. The growth temperature for AlGaN growth is therefore only increased slightly compared to GaN growth. Instead, the enhanced nitrogen diffusion by the Ga bilayer is used to obtain smooth AlGaN layers. For thin AlGaN layers, the temperature is usually kept constant in order to reduce the interruption time between $\mathrm{GaN}$ and $\mathrm{AlGaN}$ growth to prevent contamination of the surface from the ambient $[45,46]$.

The bond strength between of $\mathrm{Al}-\mathrm{N}$ is much higher than that of Ga-N. Aluminum is therefore preferably incorporated over gallium for the applied substrate temperature range. This means that first every $\mathrm{Al}$ atom binds with a free $\mathrm{N}$ atom before any Ga- $\mathrm{N}$ bonds form. The $\mathrm{Al}$ content is thus $x_{A l}=\mathrm{Al} / \mathrm{N}$ and is independent of the Ga-flux under overall metal rich conditions [44]. This incorporation ratio is independent of temperature below the decomposition temperature of GaN. The Al-flux during AlGaN growth is thus determined by the desired Al content.

The Ga-flux is chosen to provide Ga bilayer stabilized growth conditions during AlGaN growth [48]. Compared to GaN growth, the Ga-flux should be adjusted during AlGaN growth, because less Ga is incorporated over time. This implies a change of the Ga cell temperature, which results in a growth interruption. In order to avoid this stop during growth, some MBE machines are equipped with two Ga cells $[49,50]$. This allows to change the effective Ga-flux instantly.

Apart from the growth conditions, some material specific properties have to be accounted for. No segregation is expected in AlGaN [51]. However, long range ordering was observed in strained AlGaN layers even at low $\mathrm{Al}$ 
concentrations $[52,53]$. This ordering results in a superlattice of $\mathrm{Al}$-rich and Ga-rich monolayers along the c-axis. The inhomogeneity in the chemical distribution by this ordering is perpendicular to interfaces. The impact on electrons moving along interfaces, which is the subject of this work, is therefore small.

The relaxation of AlGaN layer becomes important for heterostructure growth. AlGaN is under tensile strain if grown on GaN. With increasing thickness, the strain energy rises. Above a critical thickness, the incorporation of dislocations, which reduce the strain, is energetically favored. This leads to a relaxation of the AlGaN layer, which is not desired for heterostructure growth. A graph of the expected critical thickness is given in Figure 1.20. For low Al content, relaxation is not expected for AlGaN layers thinner than $50 \mathrm{~nm}$.

\subsection{4 $\ln G a N$}

The MBE growth of InGaN is considerably more complex than that of AlGaN and different optimum growth conditions are found in literature. The bond strength of In-N is much weaker than Ga-N. InGaN thus has to be grown at lower temperatures ${ }^{2}$ than $\mathrm{GaN}$ in order to avoid decomposition. Ga is preferably incorporated over In because of the stronger bond. At elevated temperatures, this even results in the replacement of incorporated indium atoms by gallium atoms. The result is a decreased In incorporation at higher substrate temperatures, as is shown in Figure 1.21. The indium incorporation is therefore given by $x_{\ln }=1-\mathrm{Ga} / \mathrm{N}$ at temperatures below $560{ }^{\circ} \mathrm{C}$ but decreases rapidly at higher substrate temperatures [54].

2 Note that the temperatures stated in publications for the same growth conditions vary

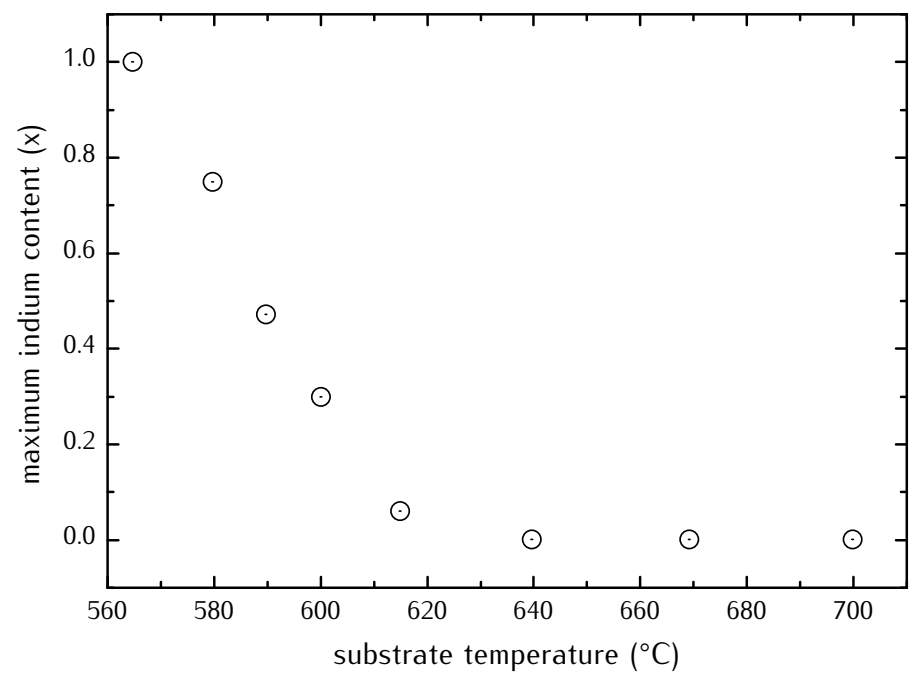

Figure 1.21: Indium incorporation over substrate temperature [54]. 
The decomposition of InGaN at higher temperatures can be counteracted by a high overpressure of active nitrogen. This allows for higher growth temperatures and stabilizes the indium content. Such growth conditions are applied for InGaN structures with high indium content of for $\ln N$ growth [24].

Other approaches utilize the enhanced nitrogen diffusion under In-rich conditions at lower temperatures aroung $450{ }^{\circ} \mathrm{C}$ [55]. However, for typical growth conditions the substrate temperature is not high enough for significant indium desorption. In-rich growth therefore produces In droplets which cause inhomogeneous morphologies and indium contents in the growing film [56]. Some groups have recently reported the growth of InGaN under In bilayer stabilized conditions which prevents the formation of droplets [57, 58, 59]. However, this is limited to lower indium contents. Growth in this regime requires sufficient indium desorption rates. This is only achieved at elevated temperatures (above $590^{\circ} \mathrm{C}$ ), where the indium incorporation strongly depends on the substrate temperature. This growth regime has not been studied in detail yet and was applied in this work.

In any of the stated approaches, the homogeneous distribution of In is an issue. InGaN has a strong tendency to segregate as shown in Figure 1.22. It was however reported that the miscibility gap decreases and shifts towards higher indium content for strained layers $[51,60]$. The experimental verification is under discussion. No large indium rich clusters have been observed for optimized growth. Many groups, however, observed small clusters by TEM. This is argued to be segregation induced by the electron beam of TEM itself [61] and is thus not accepted as a proof of segregation by others.

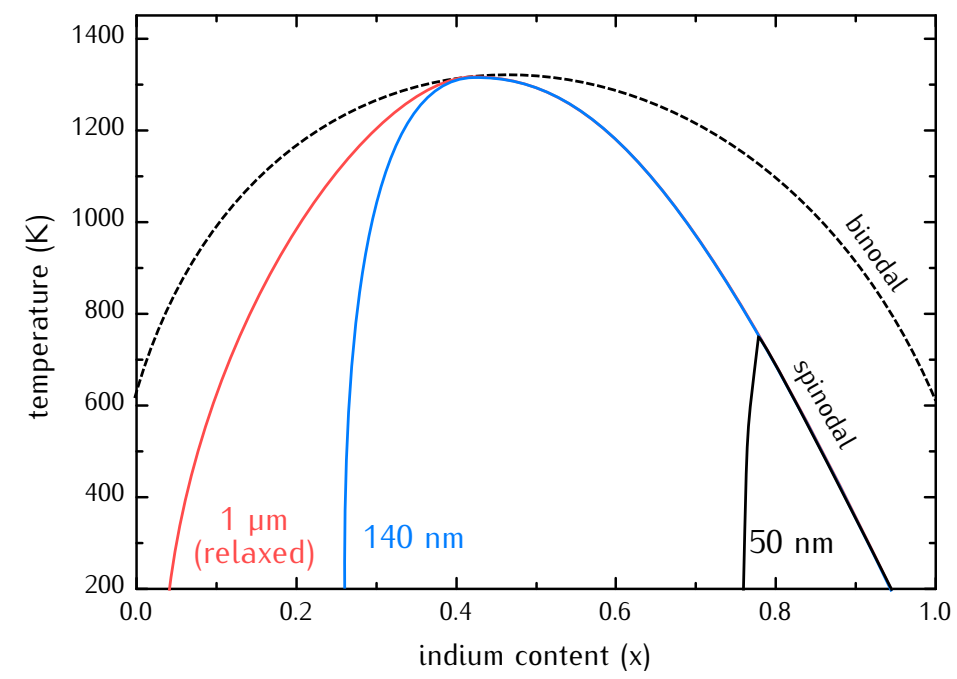

Figure 1.22: Phase diagram of InGaN (0001) for different layer thicknesses [51]. The miscibility gap for thinner layers is smaller and shifted towards $\ln \mathrm{N}$. 


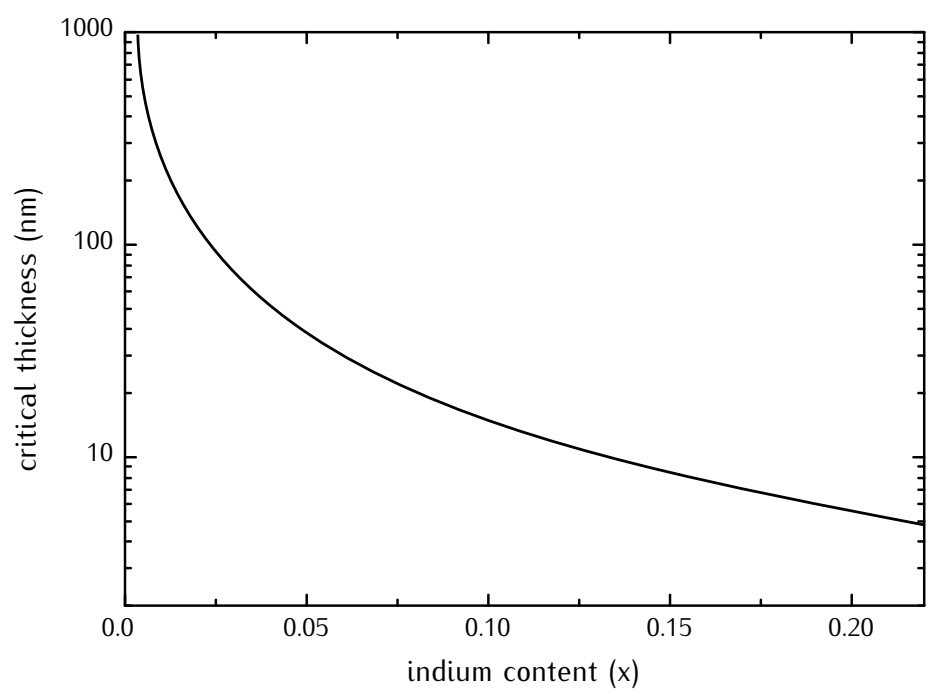

Figure 1.23: Critical thickness for InGaN relaxation over InGaN concentration for low In contents [40]. For a layer thickness of $10 \mathrm{~nm}$ relaxation is expected above $x_{\ln }=0.15$.

The critical thickness for relaxation of InGaN layers is given in Figure 1.23. Compared to AlGaN of the same Ga content, relaxation is expected at thinner layers $[40,41]$. Relaxation thus has to be considered for GaN/InGaN heterostructure growth.

Another issue, often reported on, is surface segregation. Surface segregation means the exchange of atoms between subsurface and surface layers [63]. It was shown that In atoms from the subsurface layer will exchange with

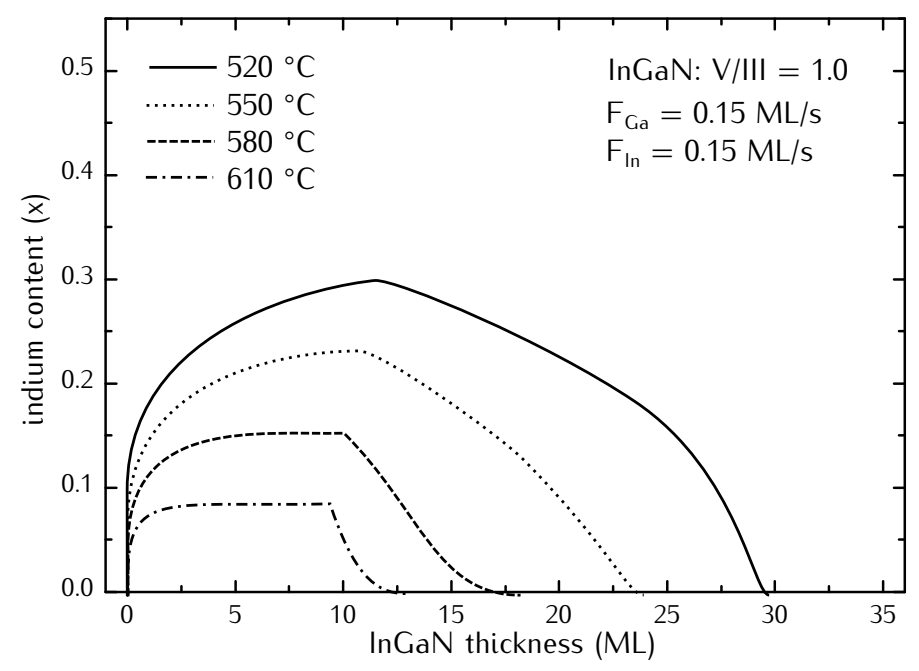

Figure 1.24: Concentration profile of $x_{/ n}$ over thickness for different substrate temperatures grown at stoichiometric conditions [62]. Surface segregation induces an increase of $x_{\ln }$ after growth is started by accumulating indium. A slow decrease of $x_{/ n}$ is observed after $\ln$ is switched of due to the incorporation of accumulated indium. 


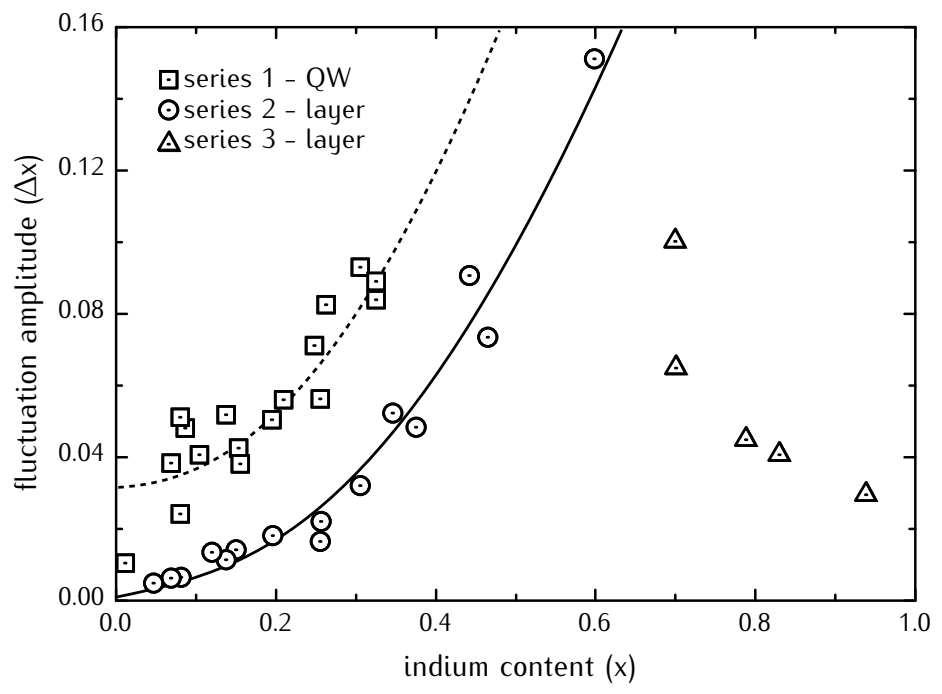

Figure 1.25: Indium fluctuation amplitude over indium content [65].

Ga atoms from the surface layer because of the different bond strength of In-N and Ga-N. Observations by STM revealed one monolayer of In accumulated on top of an InGaN layer due to this effect [64]. If InGaN is grown at temperatures with low desorption rates, at $\mathrm{N}$-rich or stoichiometric conditions, the segregated indium will represent an additional supply of indium for growth. The incorporated amount of indium will then increase over time and decay after In is switched off as shown in Figure 1.24 [62]. This onset and tailing effect was not observed in this work.

One problem for InGaN heterostructure growth are fluctuations in the indium content, which is independent of the applied growth technique. This should not to be confused with segregation, as no In-rich clusters are observed. In fact, the indium distributes inhomogeneously, as was observed by TEM [65]. As shown in Figure 1.25, in an InGaN film containing $12 \%$ of indium, areas with $10 \%$ and $14 \%$ have been found. These fluctuations have different consequences for the recombination of electrons and holes in LEDs and the transport of electrons along the interface, which was studied in this work. Because the band gap is smaller in regions with higher indium content, photons are mainly generated from recombination in these areas. The recombination at other non radiative defects is therefore reduced [66]. For transport along the interface in a heterostrucutre, the fluctuations are an additional source of scattering, as will be shown in this work. 


\section{RHEED}

Reflection high-energy electron diffraction is the most applied in-situ method in MBE growth today. In this work RHEED was used to optimize different aspects of growth. The following sections describe the background of this technique, which is necessary to understand the observed data. Furthermore, the applied analyzing methods are demonstrated in detail.

\subsection{FUNDAMENTALS}

Reflection high-energy electron diffraction (RHEED) offers real time analysis of the growing interface without interrupting the growth process. This is because the small incident angle permits the positioning of the effusion cells almost normal to the surface of a growing sample (see Figure 1.1). The small inclination also results in a high surface sensitivity which allows analyzing the growth front. Many different pieces of information about the film quality and growth conditions can be obtained by this method. In the following, only the aspects necessary to understand the observed data are introduced briefly. For a deeper understanding and other applications, two resources are recommended $[67,68]$.

The basic RHEED setup consists of an electron gun for high energy electrons (14 keV in this work), a phosphorus screen to visualize the diffraction pattern and a camera for analysis. The electrons incline under a small angle $<3^{\circ}$ and scatter elastically [67] from the surface which results in a diffraction pattern observed on the screen. The electron beam can be focused and deflected by magnetic coils. Because of the small inclination, the beam interacts with the surface over several millimeters. It thus probes a large slab of the sample. The sample can be rotated, which allows to analyze different directions. In addition to the diffraction pattern, a spot of the directly reflected electron beam, the so called specular reflection spot, is observed. The intensity of this spot is addressed in this work if the intensity of RHEED is discussed. 
Figure 2.1

Comparison of the reciprocal lattice for a $3 \mathrm{D}$ cubic lattice to reciprocal rods from a square net.

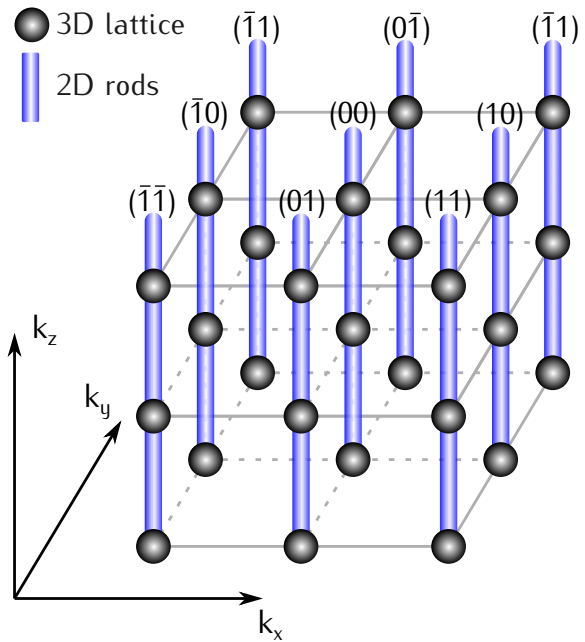

The RHEED diffraction patterns are different from other electron diffraction techniques. The high energy of the electrons and the small inclination result in a small penetration depth of only a few monolayers [69]. Therefore, diffraction has to be treated for a two dimensional surface. The reduction from three to two dimensions results in a degeneracy in reciprocal space because no diffraction condition exists normal to the surface. Reciprocal lattice points thus degenerate into infinite rods based on a 2D reciprocal net as illustrated in Figure 2.1.

The interference pattern observed on the phosphorous screen is given by the Laue condition. Constructive interference is observed if the difference of the wave vector of an incident electron $\overrightarrow{k_{0}}$ and that of the diffracted electron $\overrightarrow{k^{\prime}}$ are equal to a reciprocal lattice vector $\vec{K}$ :

$$
\overrightarrow{k_{0}}-\overrightarrow{k^{\prime}}=\vec{K}
$$

For elastic scattering where $\left|\overrightarrow{k_{0}}\right|=\left|\overrightarrow{k^{\prime}}\right|$, this can be visualized by the Ewald sphere. The radius of the sphere is $\left|\overrightarrow{k_{0}}\right|$ and is large compared to the rod spacing for high energies ${ }^{1}$. The Laue condition implies that constructive interference occurs if the Ewald sphere intersects with a reciprocal lattice rod. This is illustrated in Figure 2.2. The resulting diffraction pattern observed on the screen consists of streaky reflexes on so called Laue rings.

It should be noted that elastic scattering from only the first monolayer is not sufficient for a full quantitative description of the intensity and resulting patterns. Inelastic processes, as well as scattering from multiple atomic layers, would have to be accounted for. This is neglected in this work because it has no effect on the presented analyzing methods.

$1\left|\overrightarrow{k_{0}}\right|=\frac{2 \pi}{\lambda}=630 \mathrm{~nm}^{-1}$ at $14 \mathrm{keV}$ 

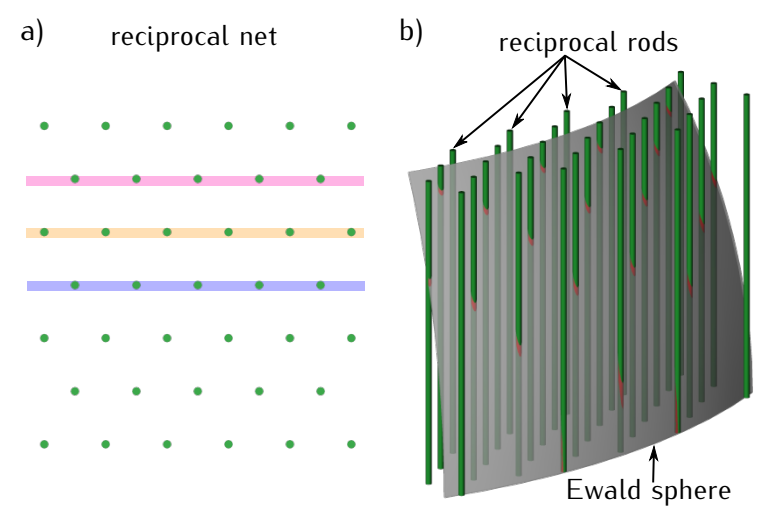

c)

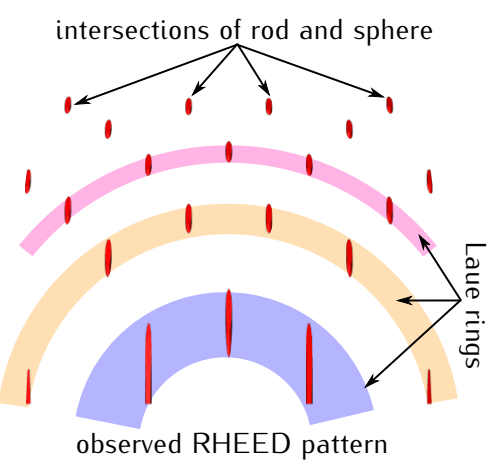

Figure 2.2: Construction of the RHEED pattern. a) reciprocal net from a hexagonal surface, b) intersection of reciprocal lattice rods with an Ewald sphere and c) resulting RHEED pattern with streaky reflexes and Laue rings.

\subsubsection{Patterns}

A 2D surface should result in a RHEED pattern built up of points, because infinitesimal thin lattice rods intersect with the Ewald sphere at one point only. This is not the case under realistic conditions. The electron beam will not be fully monochrome, and the shell of the Ewald sphere has a finite extension. Furthermore, the finite size of the crystal, strong lattice vibrations at high temperatures and atomic steps on the surface cause a broadening of the lattice rods. Both effects result in a larger cross section between rods and sphere. Real RHEED patterns for smooth surfaces therefore consist of streaky reflexes as shown in Figure 2.3 a).

A spotty pattern is observed for rough surfaces as shown in Figure 2.3 b). Because the diffraction from a $2 \mathrm{D}$ lattice is not applicable for a $3 \mathrm{D}$ surface, the lattice rods form segments according to the $3 \mathrm{D}$ reciprocal lattice for the bulk material (Figure 2.4). The increased roughness also causes a broadening of the segments which results in a bigger overlap with the Ewald sphere. The spots of a rough surface are therefore broader than the streaks observed for a smooth surface.
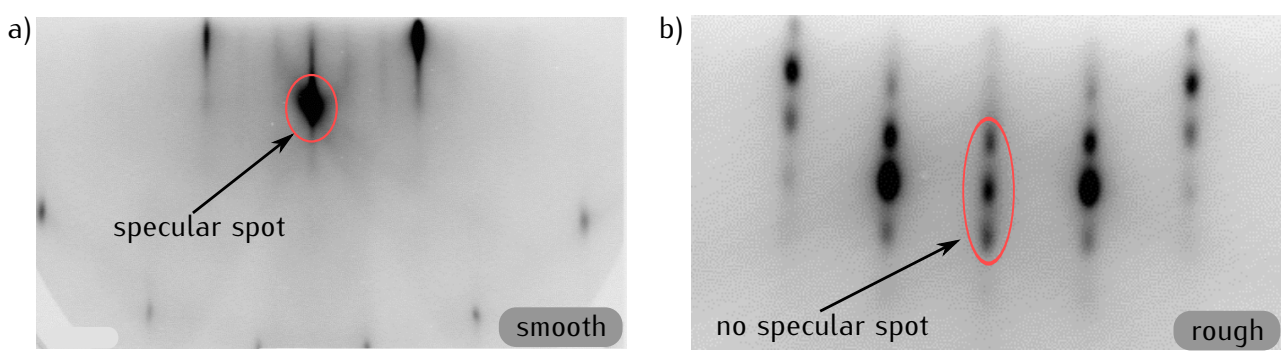

Figure 2.3: Comparison of a) a streaky RHEED pattern of a smooth surface and b) a spotty pattern of a rough surface. Note that no specular spot is observed in b). 
$2 \mathrm{D}$

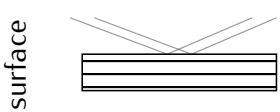

总

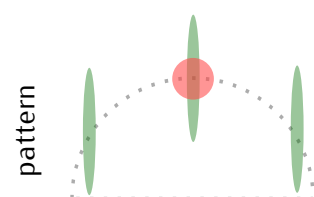

quasi $2 \mathrm{D}$

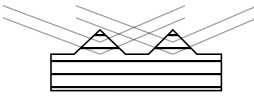

1

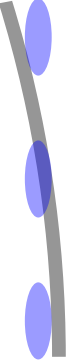

$3 \mathrm{D}$
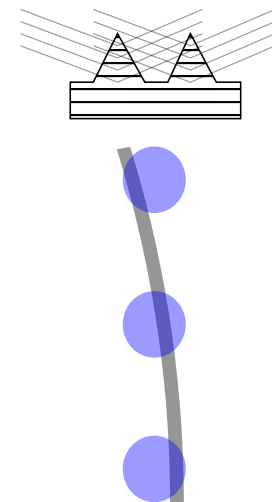

polycrystaline
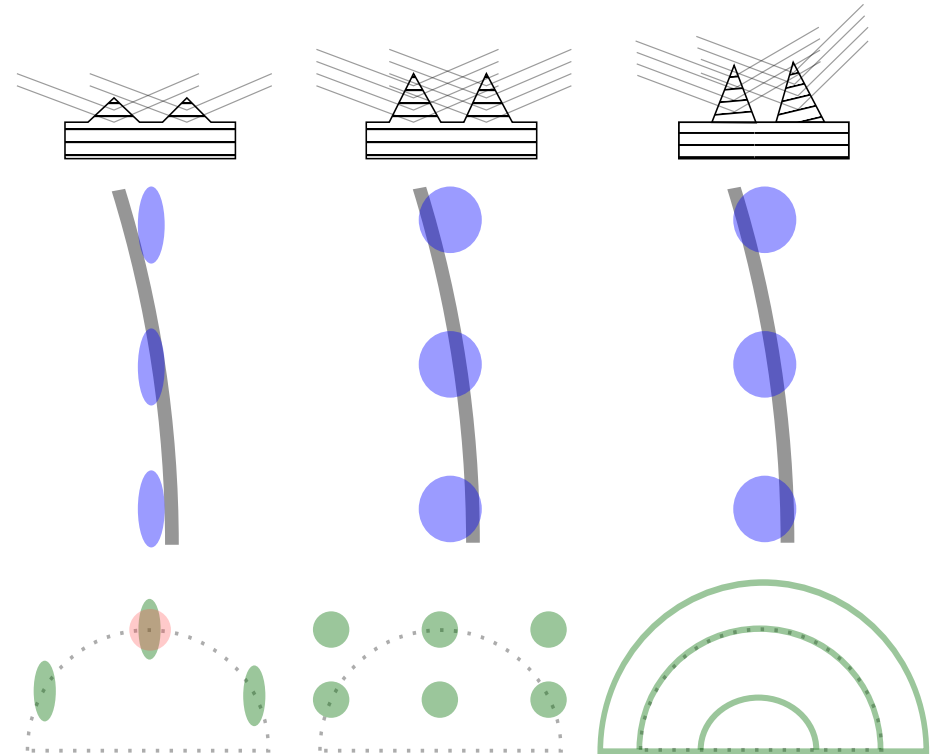

Figure 2.4: Origin of different RHEED patterns for a 2D, quasi 2D, 3D and polystralline surface [70]. The diffraction results in different reciprocal reflexes (blue). The intersection with the Ewald sphere (grey) produces characteristic RHEED patterns (green) for the different surfaces. The specular spot is indicated in red and looses intensity for rougher surfaces.

The pattern modifies further for even rougher surfaces, e.g. for islands or nanowires. The electron beam is no longer directly reflected but rather transmitted through the nanostructures. This results in a spotty pattern and the specular spot will lose intensity until fully gone (Figure 2.4 and Figure 2.3 b)). If, in addition, the islands are not oriented towards each other, the spots will form circles.

It is now clear, that RHEED can be used to distinguish between smooth and rough surfaces during growth. However, no conclusion on the morphology can be drawn from streaky RHEED patterns and the surface has to be probed exsitu. Atomic force micrographs of two samples with almost identical RHEED patterns are shown in Figure 2.5. Both show a similar roughness but a entirely different morphology.

\subsubsection{Reconstructions}

In addition to the regular patterns discussed in the previous section, other reflexes induced by surface reconstructions can be observed as shown in Figure 2.6. These reflexes are only visible for smooth surfaces that are not covered by liquid metallic adlayers. There are several reasons for a change in symmetry at the surface such as dangling bond reconfiguration, regular arrangement of adatoms or ordering in alloys. If the origin of a modified 

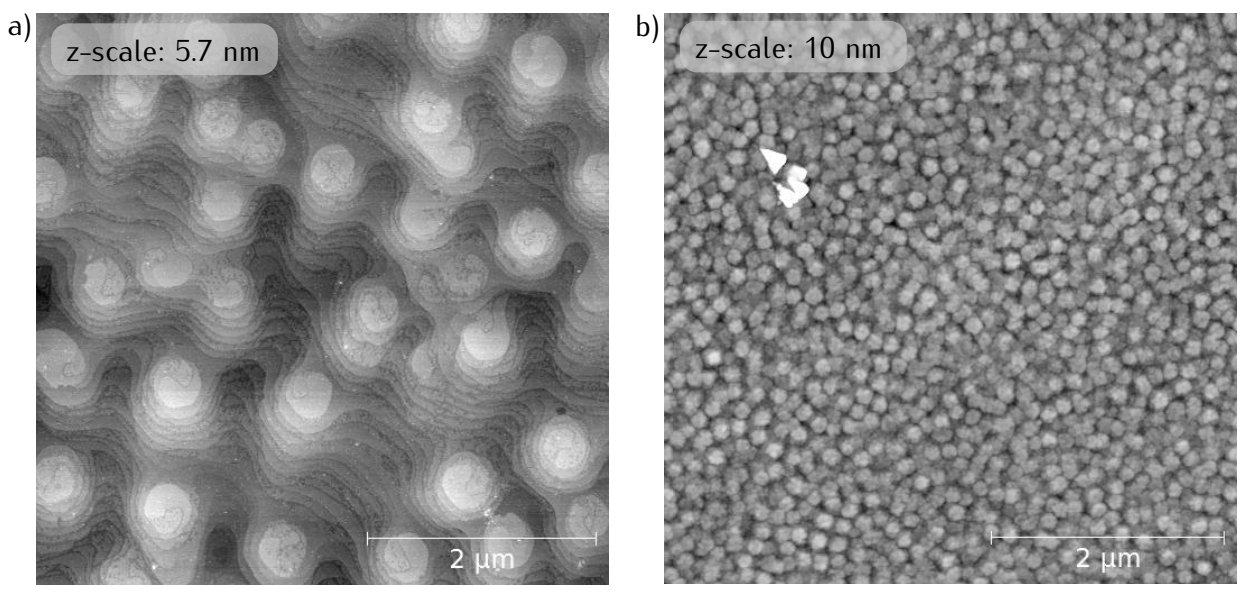

Figure 2.5: Atomic force micrographs for two samples which both showed streaky RHEED patterns. The morphology is different and could not be distinguished by RHEED patterns.

surface is known, the observation of RHEED reconstruction has significant implications for growth. Some examples are given here.

The best known reconstruction is probably the $7 \times 7$ of on Si (111). It forms at high temperatures under UHV conditions and occurs only after the removal of the native oxide [71]. The observation of this reconstruction thus indicates the successful removal of the oxide prior to growth. $\mathrm{On} \mathrm{SiC}$, a $2 \times 2$ reconstruction is observed, if the oxide is still present and a $1 \times 1$ represents a clean $\mathrm{SiC}$ surface [72]. During the growth of MnGa, the reconstruction indicates the stoichiometry of the growing phase [73]. GaN shows various reconstructions for nitrogen and gallium rich surfaces at different temperatures which can be used to determine growth conditions [74]. In this work, the $\sqrt{3} \times \sqrt{3} \mathrm{R} 30^{\circ}$ reconstruction of indium on $\operatorname{InGaN}$ and GaN surfaces was found to correlate with degradation of the surface under high temperature growth conditions

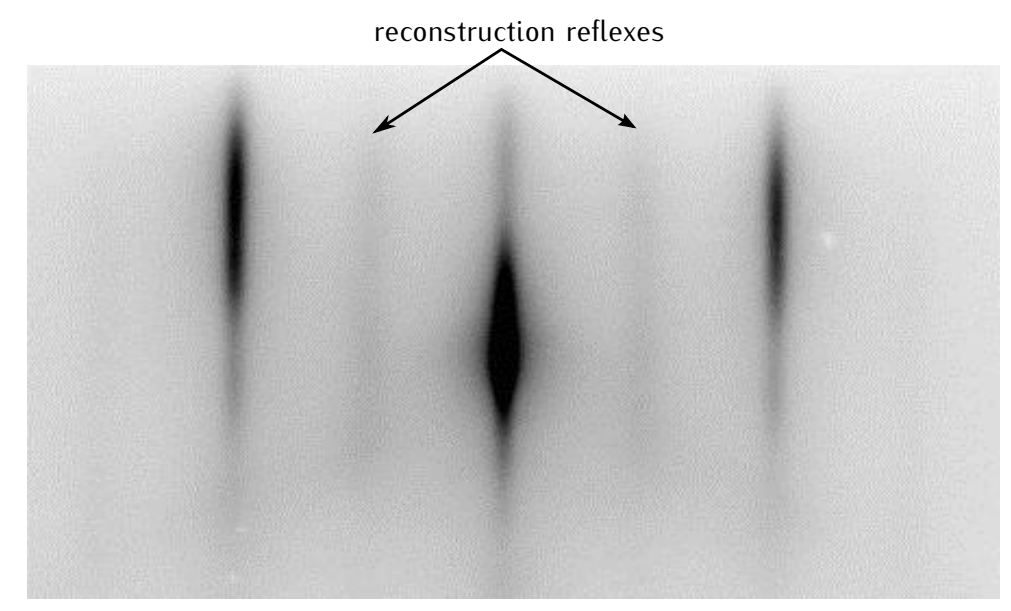

Figure 2.6: RHEED pattern of a $2 \times 1$ reconstructed GaN (0001) surface with reconstruction reflexes. 


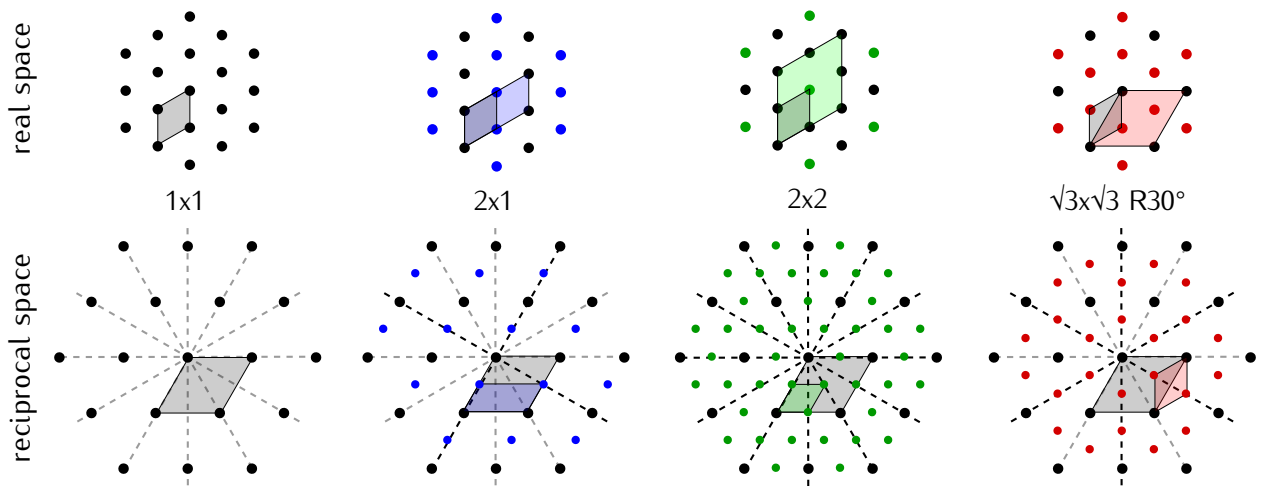

Figure 2.7: Comparison of different reconstructions. The change in the unit cell is indicated in real and reciprocal space. The additional spots in reciprocal space are characteristic for the reconstruction. The dashed lines in reciprocal space represent the high symmetry directions in RHEED.

(see Section 6.2).

The relationship between observed RHEED patterns in reciprocal space and the actual surface reconstruction in real space is not straight forward. Figure 2.7 gives three examples for reconstructions of a hexagonal surface. A $2 \times 2$ reconstruction adds one reflex in all high symmetry directions. For a $2 \times 1$ reconstruction, additional signals are only observed every $90^{\circ}$. The $\sqrt{3} \times \sqrt{3} \mathrm{R} 30^{\circ}$ results in features only for the $(1 \overline{10})$-direction ${ }^{2}$. This clarifies that one direction holds not enough information to identify reconstructions and at least two highly symmetric directions have to be monitored. RHEED radar scans (see Section 2.2.1) provide clearer results.

\subsubsection{Indication of directions}

A RHEED pattern depends on the angle between electron beam and lattice planes of the crystal and so on sample rotation. The same pattern is observed

$2 \mathrm{R}^{\circ} 0^{\circ}$ indicates a rotation of the unit cell by $30^{\circ}$
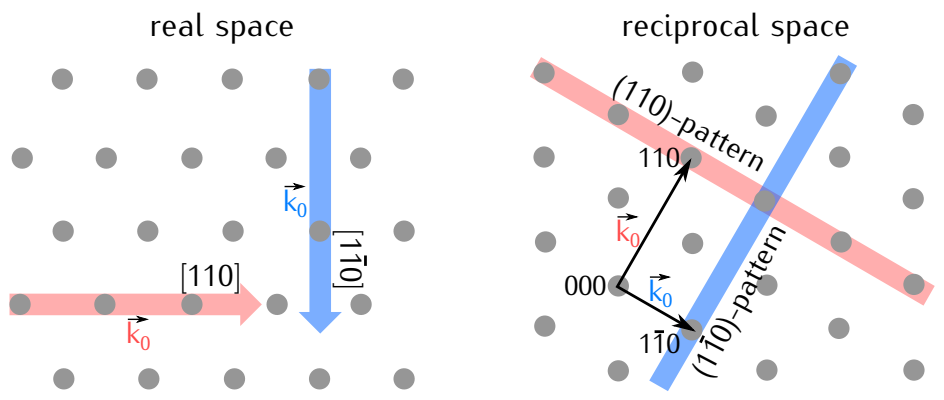

Figure 2.8: Indexing of the the two high symmetric directions of a hexagonal lattice. The reflexes of the (110) pattern are closer together compared to the (11̄0) pattern. 
after rotation by a specific angle, depending on the crystal symmetry. For the hexagonal surface of c-plane wurtzite GaN, this occurs every $60^{\circ}$.

Miller indices are used to index different patterns as illustrated in Figure 2.8. The direction of the electron beam in real space is represented by the vector $\overrightarrow{k_{0}}$ in reciprocal space. The Ewalds sphere is perpendicular to $\overrightarrow{k_{0}}$ and the resulting pattern is index according to the Miller indices of $\overrightarrow{k_{0}}$. In principle, every pattern has its own index, but a convention ascribes the (110) and (11) direction to the two high symmetry GaN patterns.

\subsubsection{Spacing between reflexes}

The distance between the observed RHEED reflexes gives another information. It represents the spacing between reciprocal lattice planes and is inversely proportional to the in-plane lattice constant in real space. The spacing between reflexes thus holds information about the strain or the chemical composition of an alloy. In this work, only pseudomorphic layers are investigated. Pseudomorphic layers have matching lattice constants in the plane of inclination, and there should be no difference observed, e.g. for AlGaN or InGaN grown on GaN.

Still significant changes were found for growth under metal rich conditions as shown in Figure 2.9. The observed strain changes as soon as metal is deposited. After the adlayer is gone, the strain recovers to the original value. This is an elastic phenomenon induced by the excess metal [75]. This change in spacing of the RHEED reflexes can therefore be used to monitor the metal coverage during pseudomorphic growth.

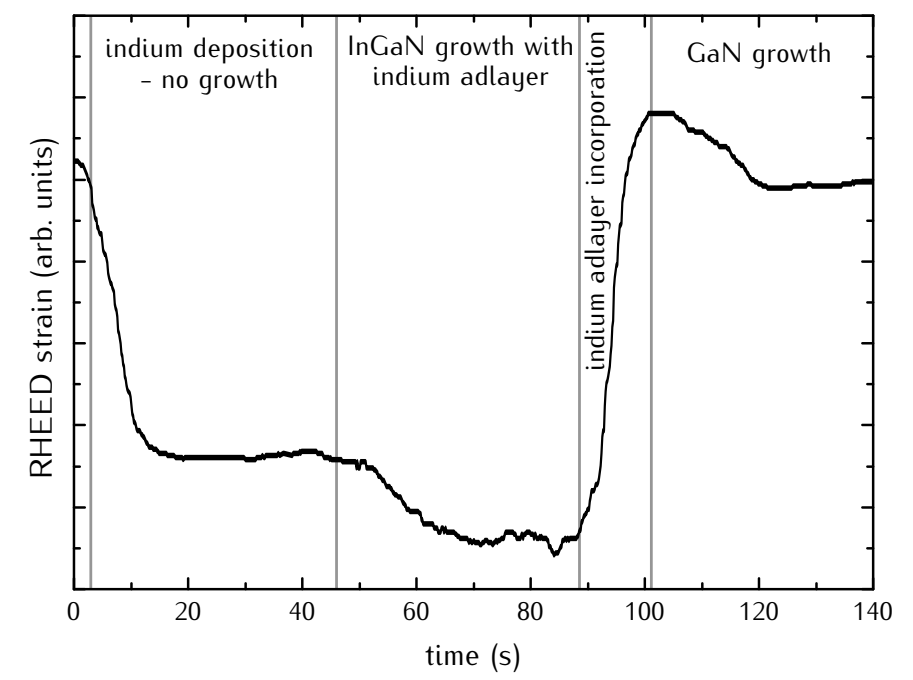

Figure 2.9: Spacing of RHEED reflexes during InGaN under a indium adlayer. The reduced strain during metal deposition is an elastic process. During InGaN growth the strain reduces only slightly. After the incorporation of the indium adlayer, the strain recovers to it original values. 


\subsubsection{RRS: RHEED Radar Scans}

Different directions of inclination have to be monitored for a complete characterization of the surface as was mentioned in Section 2.1.2. RHEED radar scans (RSS) are one way to do so. In this analysis, the substrate rotates and the pattern is captured every degree or less. The intensity profile of a slim frame is then calculated for each rotation angle ${ }^{3}$. During the rotation, all reflexes will move in and out of the frame as illustrated in Figure 2.10. An RRS is then a polar plot of the profile data for every angle. It represents the reciprocal net of the surface and is comparable to low-energy electron diffraction (LEED) images. This method is used to identify different orientations, complex reconstructions and epitaxial relations [76]. An example, comparing a GaN (0001) without reconstruction to a InGaN (0001) surface with a $\sqrt{3} \times \sqrt{3} \mathrm{R} 30^{\circ}$, is given in Figure 2.11. The intensity decreases every $90^{\circ}$ because the sample holder covers parts of the surface.

\subsubsection{Growth rate by RHEED oscillations}

Oscillations in the RHEED intensity are observed during 2D nucleation growth. In this growth mode, two dimensional nuclei form on the surface and keep growing laterally until they merge. Afterwards, new nuclei form and the process starts over. The RHEED intensity changes periodically during this growth cycle as illustrated in Figure 2.12.

The reflection is highest for a closed monolayer. The surface gets rougher

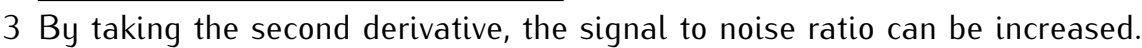

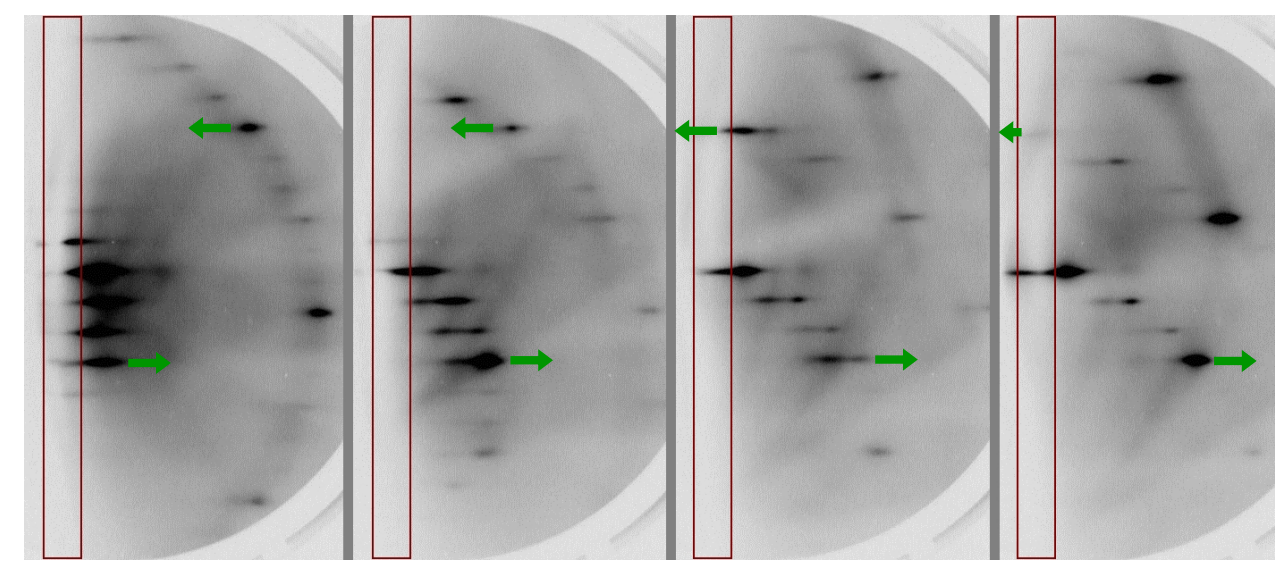

Figure 2.10: RSS construction: A RHEED pattern in shown for different sample rotations. The intensity in the red box is used to construct the RSS images. The reflexes move in and out of the box during sample rotation, as is indicated for two reflexes. 

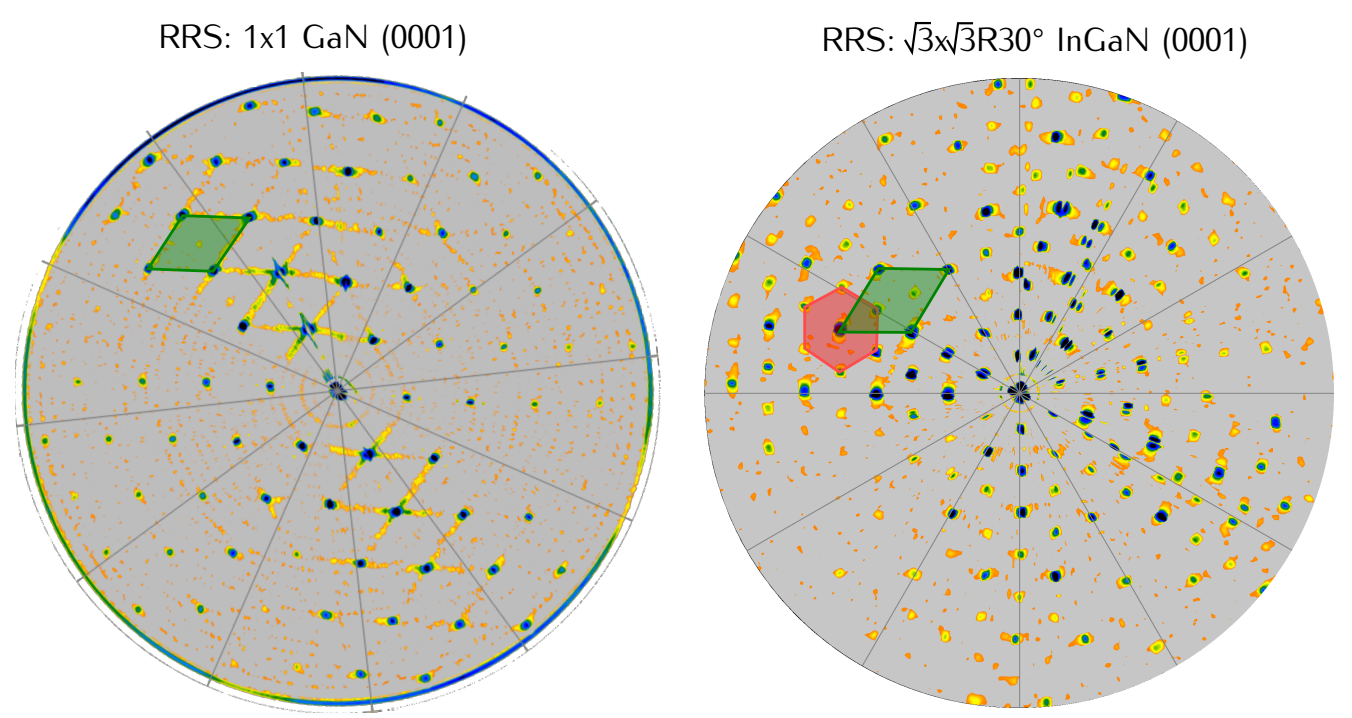

Figure 2.11: RRS of $1 \times 1 \mathrm{GaN}(0001)$ and $\sqrt{3} \times \sqrt{3} \mathrm{R} 30^{\circ} \operatorname{InGaN}(0001)$. The unreconstructed unit cell in indicated in green, the reconstruction reflexes in red. For comparison see Figure 2.7.

when nuclei form and the RHEED intensity decreases. If half of the surface is covered with nuclei, the roughness will be maximal and thus the RHEED intensity minimal. As the growth continues and the monolayer closes, and the intensity rises again. This cycle leads to oscillations. In reality, the nucleation will restart on incomplete monolayers and the surface will eventually get rougher over time. The oscillations therefore decrease in amplitude for longer growth periods. An example of RHEED oscillations is shown in Figure 2.13 b).

The frequency of the oscillations is the number of monolayers grown per time. This can be converted into the actual growth rate of the material if the lattice constant is known. The amplitude depends on the scattering cross section of the material which decreases from AIN over GaN to $\ln N$. Growth oscillations

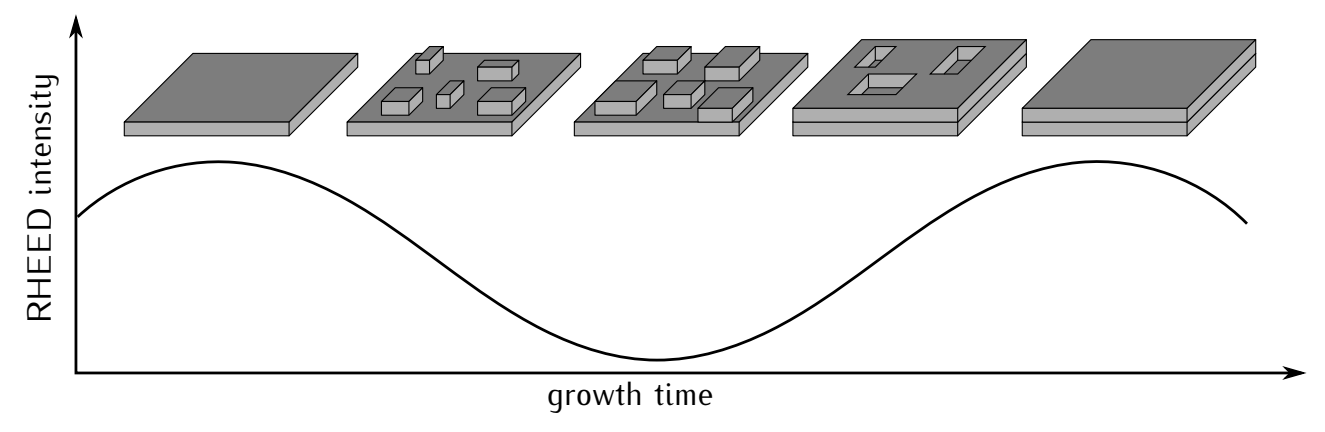

Figure 2.12: Origin of growth oscillations in the RHEED intensity. During 2D nucleation growth, the coverage of the surface changes periodically. The RHEED intensity is maximal for fully closed layers and minimal for half closed layers. This results in a oscillation over growth time. 
thus are best observed for AIN and GaN growth. The observation during InN growth is difficult because of the low intensity amplitude amplitude. The 2D nucleation growth mode is observed close to stoichiometric growth conditions for nitride growth. Nitrogen rich conditions often result in rough surfaces while step growth is present under metal rich conditions. For the latter, the growth of layers continues from existing steps on the surface, and the roughness does not change over time. This growth mode results in the best morphologies. Therefore, no RHEED oscillations are observed for optimized growth conditions but are used for calibration measurements.

\subsubsection{Determination of stoichiometry and III/V ratios}

The stoichiometry of a binary material is an important parameter. It is defined as the unity ratio of two growth components. It distinguishes $\mathrm{N}$-rich from metal-rich conditions and is used to estimate the concentrations in ternary alloy growth. In addition, it can be employed to determine the III/V ratio which is used as a measure for the metal fluxes in this work. The stoichiometry should thus be determined for all binary materials.

In order to determine the stoichiometry of a metal with nitrogen, the nitrogen flux is kept constant as the metal flux is varied during growth. A characteristic difference in RHEED intensity trends then gives the stoichiometric flux. For GaN, this is best done by monitoring the desorption after growth as shown in Figure 2.13 a). Growth oscillations are observed during growth slightly above stoichiometry. The intensity increases slightly after growth because accumulated metal desorbs. The same effect can be induced by nitridation if the desorption is insufficient at low growth temperature. At stoichiometric conditions, the intensity remains constant after growth because no metal accumulated. Under $\mathrm{N}$-rich conditions, the surface gets rougher and a spotty pattern is observed.

The stoichiometry of AIN can be determined from growth oscillations. The amplitude of the oscillations is higher for AIN compared to GaN and strong oscillations are observed around stoichiometric conditions as shown in Figure 2.13 b). Al-rich growth close to stoichiometric conditions damps the intensity oscillations because of accumulating metal. N-rich growth roughens the surface and oscillations are damped. The damping is minimal at the stoichiometry and RHEED oscillations can be observed for a longer period of time.

The presented methods provide the metal flux for stoichiometric growth. This can be used as a measure for the effective nitrogen flux which can not be gained directly. At low temperatures, the desorption rate of metals and nitrogen is negligible compared to the growth rate. Furthermore, the sticking coefficients of the individual components is close to unity [22]. This implies that every atom reaching the surface is adsorbed and incorporated. The metal flux is therefore equal to the flux of nitrogen atoms that can be 

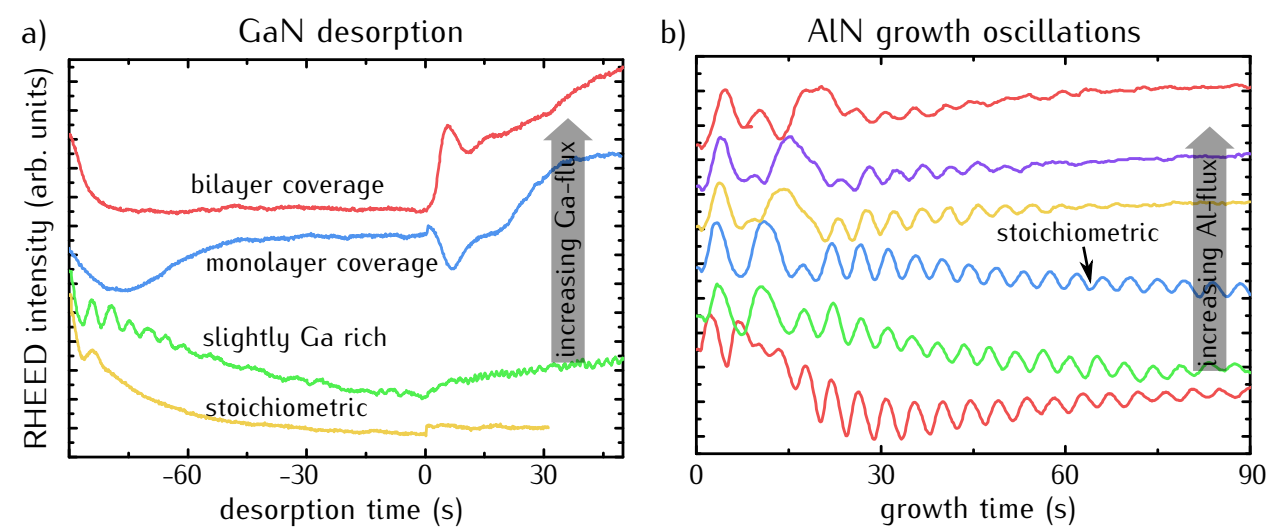

Figure 2.13: Determination of stoichiometry by a) desorption of the Ga adlayer and b) growth oscillations during AIN growth. The nitrogen flux is fixes and the metal fluxes are varied. $\mathrm{Ga} / \mathrm{N}=1$ : no desorption. $\mathrm{Al} / \mathrm{N}=1$ : most oscillation cycles.

incorporated and $\mathrm{III}=\mathrm{N}$ for stoichiometric conditions at low temperatures ${ }^{4}$. The III/N ratio for different metal fluxes under constant nitrogen conditions can then be calculated from the metal flux calibrated by a beam equivalent pressure. In this work, this is used as a measure for metal fluxes.

\subsubsection{Monitoring metal bilayer desorption}

One key factor for MBE growth of nitrides is the metal coverage (see Section 1.4). For Ga- and In-rich growth three different regimes are found at sufficient high temperature depending on the metal flux. Above stoichiometry, one liquid monolayer of metal starts to accumulate. A stable bilayer forms for a wide range of higher metal supplies. At very high fluxes, the metal accumulates in the form of droplets. Different crystal qualities are achieved for the different metal coverages (see Section 1.5.2). A precise knowledge of the conditions for which the regimes are present is therefore essential for growth optimization. This can be monitored by the RHEED intensity.

A quantitative method to determine the metal coverage is to monitor the time between growth termination and increased RHEED intensity for various Ga fluxes as was shown in Figure 1.18 [32]. Gallium or indium adlayers are liquid at typical growth temperatures. This disordered layer causes a damping of the electron diffraction from the underlying crystal, which reduces the RHEED intensity. The intensity thus decreases during metal accumulation and increases during desorption. The desorption time is hence proportional to the metal coverage. Monitoring the desorption time is a thorough approach to identify a range of metal fluxes for which a stable bilayer is present on the surface. This approach requires measurements for many metal fluxes for satisfying results.

4 the overall nitrogen flux will be higher due to non incorporable components 
The bilayer regime can also be identified by its characteristic RHEED intensity oscillation during desorption as shown in Figure 2.13 and Figure 2.14. The reason for the oscillation lies in the arrangement of adatoms which form the bilayer and in their different activation energies for desorption. Adatoms in direct contact with the surface order above metal atoms. Atoms in the second layer are compressed for a Ga bilayer and stretched for In bilayer. They are also more disordered than atoms in the first layer due to their liquid character. Because of the different activation energies the two layers desorb one after another. First atoms from the top layer leave the surface. During this desorption, the RHEED intensity increases because this layer is disordered and the damping of the electron beam is reduced. If the second layer is totally gone, the electron beam is reflected by the ordered layer which results in a maximum intensity. If atoms from the last layer desorp, the intensity will decrease and then increase because of the incomplete coverage similar to growth oscillations.

This oscillation during desorption can not be observed if the surface is covered with only one monolayer. For the droplet regime, the intensity trend is the same but it is delayed (Figure 2.14) because the desorption from adatoms above the bilayer is not detected. The desorption in every regime thus has a characteristic RHEED intensity trend. Different coverages are determined by comparing the RHEED intensity trends at the transitions between the regimes. This approach is quicker than the analysis of the desorption time and is used in this work.

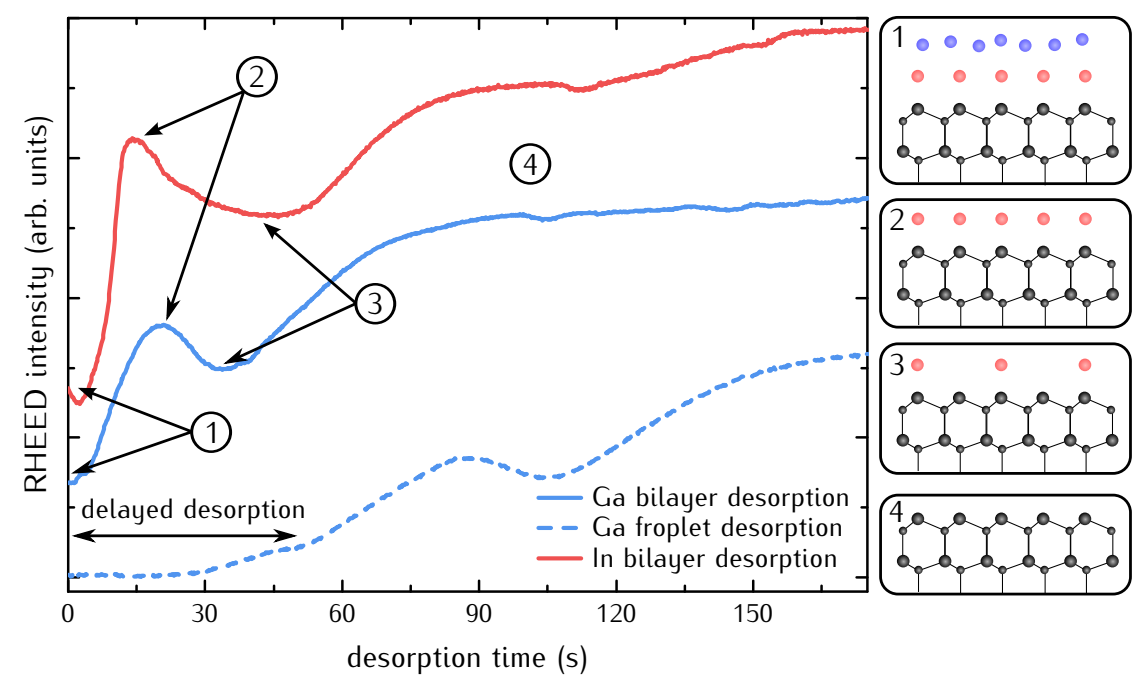

Figure 2.14: RHEED intensity during desorption after growth in the Ga bilayer, the Ga droplet and the In bilayer regime. The oscillation is characteristic for bilayer desorption. It is delayed for droplet coverage. 1: full bilayer coverage - intensity is minimal, 2: full monolayer coverage - intensity is high, but smaller than for the uncovered surface, 3: partial monolayer coverage - intensity is minimal, but higher than for bilayer coverage, 4: bare surface - intensity is maximal. 


\section{TWO DIMENSIONAL ELECTRON TRANSPORT IN III-NITRIDES}

The electron mobility of two dimensional electron gases in nitride heterostructures was investigated in this work. This chapter explains the origin of two dimensional carrier confinement in undoped GaN/AlGaN and GaN/InGaN structures. The dependence of the electron mobility on the structural quality is presented, which motivated the characterization of grown heterostructures by magnetotransport. In addition to the experimental framework of electrical measurements, different contributions to the magnetoresistance studied in this work are explained.

\subsection{D CARRIER CONFINEMENT IN III-NITRIDE HETEROSTRUCTURES}

Nitrides in the wurtzite crystal structure lack of inversion symmetry along the c-axis. Consequently, the surfaces for nitrides grown along [0001] and $[000 \overline{1}]$ directions are different as illustrated in Figure 3.1. This is indicated
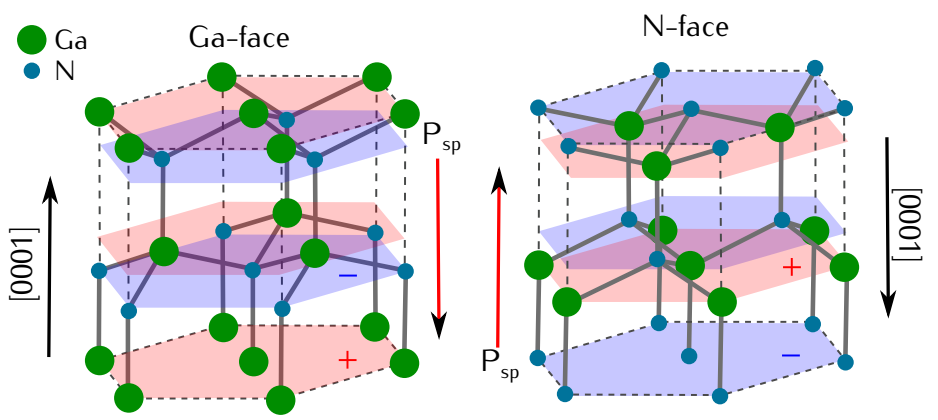

Figure 3.1: Crystal structure of wurtzite GaN for Ga-face and N-face polarity. The polar planes induces dipole charges due to the different electron affinity. They are only found perpendicular to the [0001]-direction because of the lack of inversion symmetry. The direction of the resulting spontaneous PSP polarization is indicated. 
by the notation Ga-face for GaN (0001) and N-face for GaN (0001). In this work, only metal face structures are considered.

Strong polarization effects arise from the asymmetry for both $\mathrm{N}$-face and Ga-face crystals $[77,78]$. The electron affinity for group III metals is high compared to nitrogen. Therefore, the metal-nitrogen bonds have a strong ionic character. The lattice planes along the c-direction are either pure nitrogen planes or pure gallium planes. These planes induce dipoles because of the ionic character of the bonds and are called polar planes. Whether or not the dipoles induce a macroscopic spontaneous polarization (PSP), i.e. polarization without any applied field, depends on the crystal structure.

In zincblende structures, polar planes are found along [111] directions. In this cubic structure, the four polar planes are arranged perpendicularly and the dipoles compensate each other. No PSP is therefore present in zincblende structures. In wurtzite structures, the polar planes are found along the [0001]-direction and the dipoles arrange only along this direction. However, there will be no macroscopic charge induced in an infinite crystal as each dipole charge compensates another. This is not the case for a finite crystal. Here, the dipole charges at the boundaries, i.e. surfaces or interfaces, are not compensated which results in an effective polarization charge as shown in Figure 3.2 a). The charges induce an effective polarization field in the material. This is the PSP field.

Crystals with PSP are called pyroelectric and also show piezoelectric polarization (PPE). Piezoelectric polarization means the generation of polarization fields via strain. In nitrides, the PPE originates from the strong ionic bond character. If a material is under tensile strain, the in-plane lattice constant reduces while the perpendicular lattice constant increases. The bond lengths thus change anisotropically. This results in a non uniform distribution of charges in the ionic bonds and a macroscopic PPE field.

Both polarizations have a significant impact on III-N heterostructures and are the source of two dimensional carrier confinement. Figure 3.2 shows a Ga-face GaN/AlGaN and a GaN/InGaN structure with the polarization
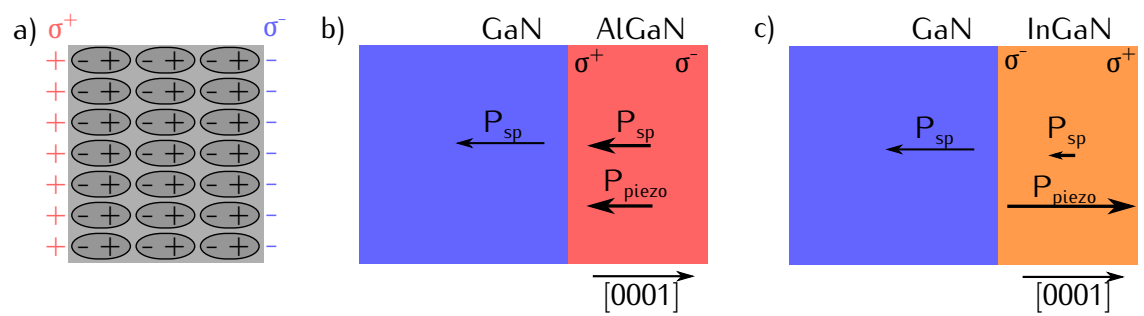

Figure 3.2: Polarization directions in GaN/AlGaN and GaN/InGaN heterostructures. a) dipole charges are uncompensated at the boundaries of the crystal and induce polarization charges $\sigma^{+}$and $\sigma^{-}$. b) PSP and PPE are parallel in GaN/AlGaN and antiparallel in GaN/lnGaN structures. The strength of polarization is indicated by the size of the arrows. 
a)
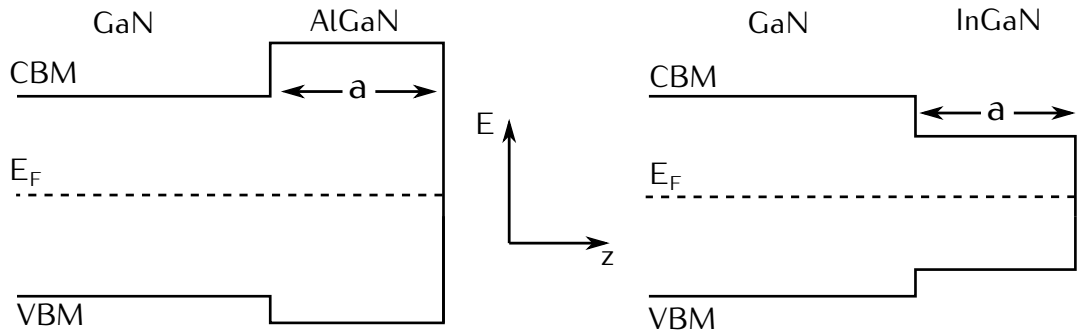

b)
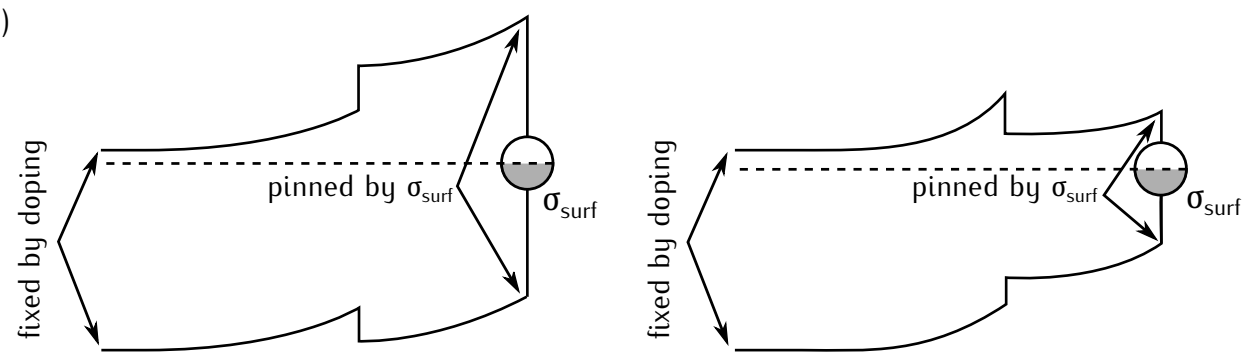

c)
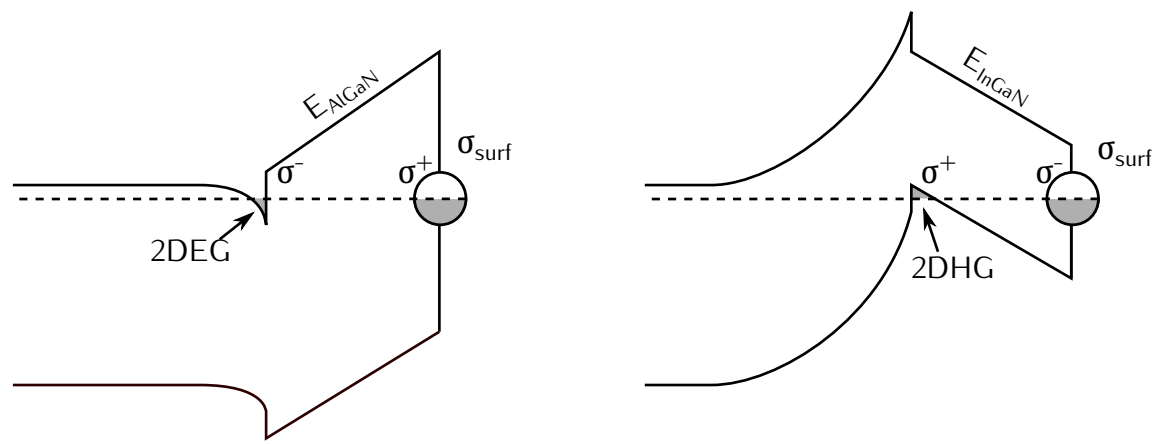

Figure 3.3: Schematic of the band profile for GaN/AIGaN and GaN/InGaN structures. a) taking only the band offsets into account, b) adding doping and surface pinning and c) adding polarization effects. Only with polarization two dimensional carrier confinement is observed.

directions indicated. The PSP field is directed from the surface towards the interface. For InGaN, its strength is smaller compared to AlGaN for the same Ga content [79, 80]. An AlGaN layer is under tensile strain on GaN (0001). In this case, the PPE has the same direction as the PSP, and their strength is comparable for low Al contents [79, 80]. An InGaN layer is compressed on GaN (0001), and the PPE is directed from the interface towards the surface. Because of the bigger lattice mismatch the absolute value of PPE is higher than that of PSP and the overall polarization field in the InGaN layer is directed towards the surface.

The influence of polarization, surface states and doping on the conduction band minimum (CBM) and the valence band maximum (VBM) profile of GaN/AlGaN and GaN/InGaN heterostructures is shown in Figure 3.3. No carrier confinement is observed if none of the three effects is taken into account. The bands are then aligned according to their band offset $E_{\text {offset }}$. Fixed boundary conditions for the Fermi level (FL) apply for heterostructures if doping and a surface potential are taken into account. In the GaN bulk, 
FL is fixed according to the doping concentration. Doping always has to be accounted for because of the high unintentional defect doping in III-nitrides [81]. The influence of a surface potential has to be considered, because of the native oxide on III-nitrides. $\mathrm{Ga}_{2} \mathrm{O}_{3}$ complexes form on $\mathrm{GaN}$ surfaces. They represent donor like surface states at $E_{\text {surf }}=1.2 \mathrm{eV}$ below CBM [82]. For $E_{F}<E_{\text {surf }}$, electrons from the surface states occupy states in the material until $E_{F}=E_{\text {surf }}$. For $E_{F}>E_{\text {surf }}$, electrons from the material occupy surfaces states until $E_{F}=E_{\text {surf }}$. In equilibrium $F L$ is thus pinned at $1.2 \mathrm{eV}$ below CBM [83]. Electrons screen the surface potential in $\mathrm{n}$-doped materials which induces a downward band bending below the surface.

Polarization fields with field strength $E_{A l G a N}$ and $E_{I n G a N}$ induce a downward tilt of the bands below the surface in AlGaN and an upward tilt in InGaN. The potential difference induced by the field between surface and interface increases with the thickness. Above a critical AlGaN thickness, CBM drops below FL [84]. For InGaN, the opposite field direction causes a rise of VBM above FL. The critical thickness $a_{c}$ depends on the polarization field strength and therefore on the composition of the material and on the pinning of $\mathrm{FL}$ :

$$
\begin{aligned}
E_{\text {surf }}-E_{\text {AlGaN }} \cdot a_{c}-E_{\text {offset }} & =E_{F} \\
E_{\text {surf }}+E_{\operatorname{lnGaN}} \cdot a_{c} & =E_{F}
\end{aligned}
$$

Electrons occupy the free states below $E_{F}$ at the GaN/AlGaN interface which causes a bend bending in the GaN. The result is a two dimensional confinement of carriers in an almost triangular well along the interface. In GaN/AlGaN structures, a two dimensional electron gas (2DEG) forms on the GaN side of the interface. In GaN/InGaN structures, a two dimensional hole gas (2DHG) forms on the InGaN side of the interface. 2DEG formation in InGaN based heterostructures is possible for structures with GaN cap layers as will be discussed in Section 6.4. The formation of 2DEGs and 2DHGs in nitride heterostructures is induced by polarization and is possible without modulated doping.

In 2DEGs and 2DHGs the carrier concentration is called sheet carrier density $n_{s}$ according to the reduced dimension. Typical values for GaN/AlGaN structures are in the order of $n_{s}=10^{13} \mathrm{~cm}^{-2}$. The typical GaN bulk electron concentration of $n=10^{17} \mathrm{~cm}^{-3}$ due to unintentional doping can only explain a sheet electron density of $n_{s}=10^{11} \mathrm{~cm}^{-2}$. An investigation of the overall neutrality condition reveals the origin of the confined electrons [85]. The charge of the two dimensional carriers is $\sigma_{2 D}=q n_{s}$. The number of dopants is negligible compared to the observed sheet carrier densities: $\sigma_{G a N} \approx 0$ and $\sigma_{A l G a N} \approx 0$. The sum of the polarization charges at the boundaries of 
the AlGaN layer is zero: $\sigma^{+}+\sigma^{-}=0$. This only leaves the surface charge $\sigma_{\text {surf }}$ as a source for the confined carriers:

$$
\begin{aligned}
\sigma_{\text {surf }}+\overbrace{\sigma^{+}+\sigma^{-}}^{=0}+\overbrace{\sigma_{A l G a N}}^{\approx 0}+\overbrace{\sigma_{\text {surf }}}^{\approx 0}-q n_{s} & =0 \\
& =q n_{s}
\end{aligned}
$$

The major part of confined carriers thus originates from the surface states, which explains the observed high densities.

\subsection{MOBILITY AND ELASTIC SCATTERING MECHANISMS}

The carrier mobility of two dimensional systems is a powerful tool to investigate the overall quality of heterostructures. It is defined as

$$
\mu=\frac{e \tau_{t r}}{m^{*}}
$$

with the effective mass $m^{*}$ and the relaxation time between two scattering events $\tau_{t r}$. The transport lifetime $\tau_{t r}$ is a combination of the relaxation times from different scattering events $\tau_{n}$ :

$$
\frac{1}{\tau_{t r}}=\sum_{n} \frac{1}{\tau_{n}}
$$

In most cases, one scattering event $\mathrm{m}$ is dominant and $\tau_{m} \ll \tau_{n}$. This scattering event limits the mobility and $\tau_{m}$ can be calculated from $\mu$ if $m^{*}$ is known. At room temperature, the scattering of carriers with phonons limits the mobility as shown in Figure 3.4. Relaxation times depending on structural properties can thus not be determined accurately from $\tau_{t r}$ at higher
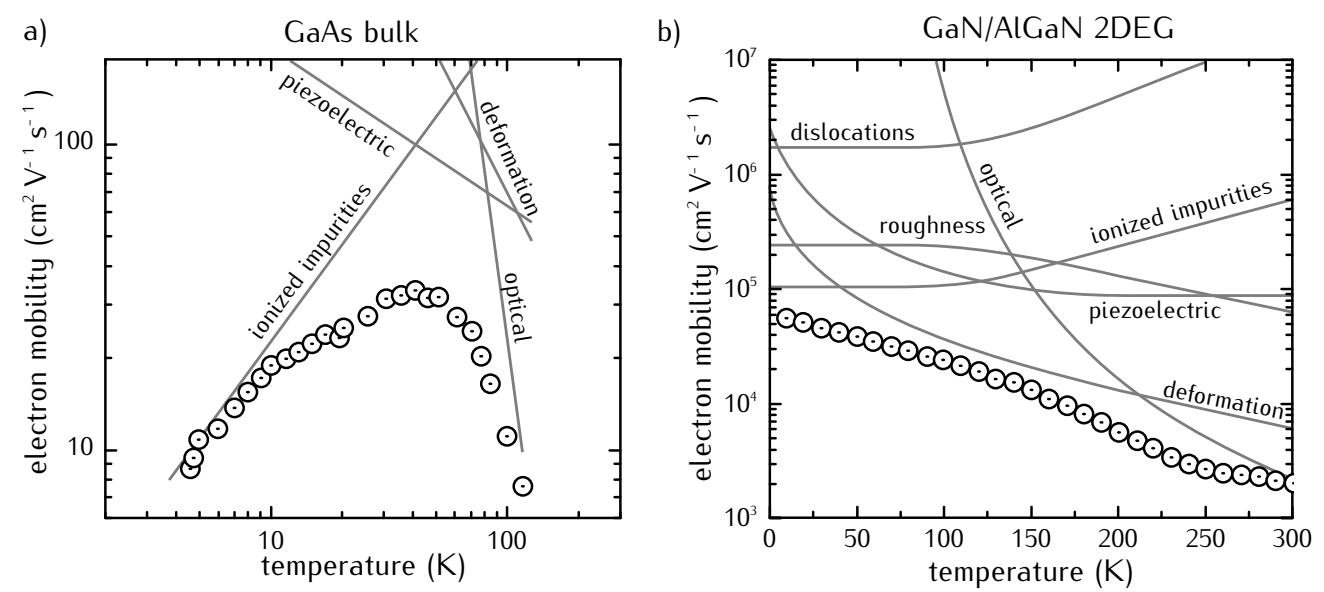

Figure 3.4: Mobility over temperature for a) GaAs bulk material [86], b) GaN/AlGaN 2DEG [87]. Scattering by phonons (optical, deformation and piezoelectric) limits $\mu$ at high temperatures. At low temperatures, $\mu$ is determined by ionized impurities in bulk material and by other defects in 2DEGs. 
temperatures. This is true for $3 \mathrm{D}$ and 2D systems. However, a 2D carrier confinement results in a remarkably different behavior at low temperatures compared to 3D transport.

The scattering by phonons is reduced considerably for lower temperatures and the mobility increases. For a 3D system, a decreased temperature also results in a stronger localization of carriers at their host atoms. Consequently, the scattering at the ionized host atoms represents the shortest relaxation time. The result is a maximum of $\mu_{b u l k}(T)$ at intermediate temperatures and a low mobility at low temperatures (Figure 3.4 a)).

The temperature dependence of $\mu$ for a 2DEG is different as shown in Figure 3.4 b). The confined carriers are separated from their host atoms and scattering by ionized impurities is therefore reduced. This results in an increased $\mu$ for all temperatures. Furthermore, the mobility does not decrease at low temperatures and other scattering mechanisms dominate $\mu$. Scattering processes that depend on structural parameters mainly limit the mobility of a 2DEG at low temperatures. The relative contributions of different scattering events to $\mu$ depend on the sheet electron density $n_{s}$. On the one hand, a larger number of electron screens charged defects more efficiently. The influence of scattering at ionized point defects and threading dislocations, which can be regarded as a line of ionized point defects [89], is reduced at high $n_{s}$. On the other hand, the penetration depth of the confined carriers into the AlGaN increases with $n_{s}$ as shown for GaN/AlGaN in Figure 3.5 a). The impact of scattering at interface irregularities and by alloy disorder in AlGaN thus increases at high $n_{s}$. These two opposing dependencies result in maximum mobilities at certain a $n_{s}$ as shown in Figure $3.5 \mathrm{~b}$ ). The sheet carrier density should have this value to achieve the highest possible mobility for a given sample quality. Because layer thicknesses and alloy compositions mainly determine $n_{s}$, the design
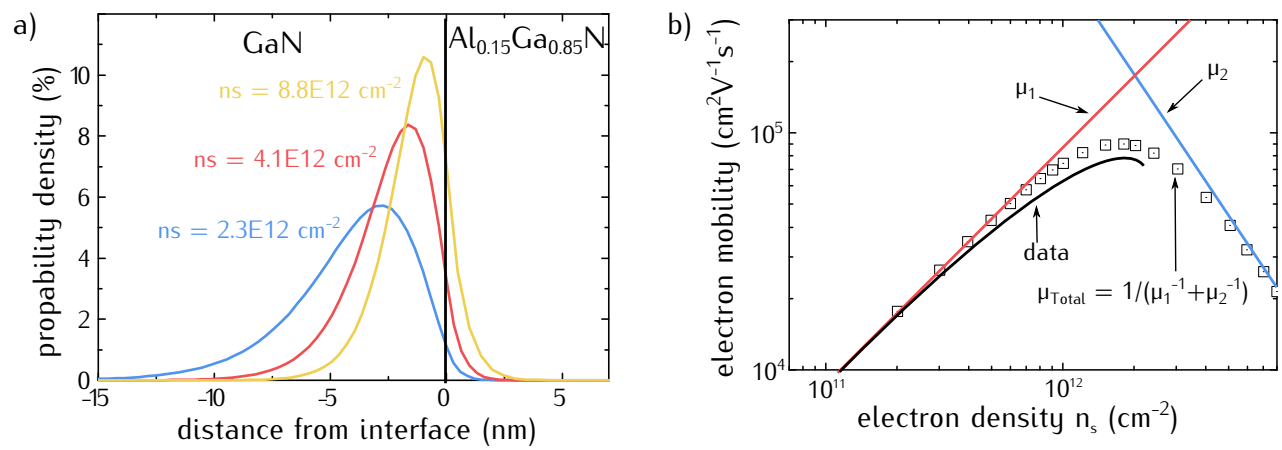

Figure 3.5: a) Calculated probability density for electrons in a GaN/AIGaN structure. The amount of electrons in AlGaN increases with increasing $n_{s}$. The impact of alloy scattering in $\mathrm{AlGaN}$ and interface roughness thus increases with $n_{s}$. b) Experimental data of $\mu\left(n_{s}\right)$ showing a maximum due to opposing $\mu\left(n_{s}\right)$ dependencies of different scattering mechanisms [88]. 


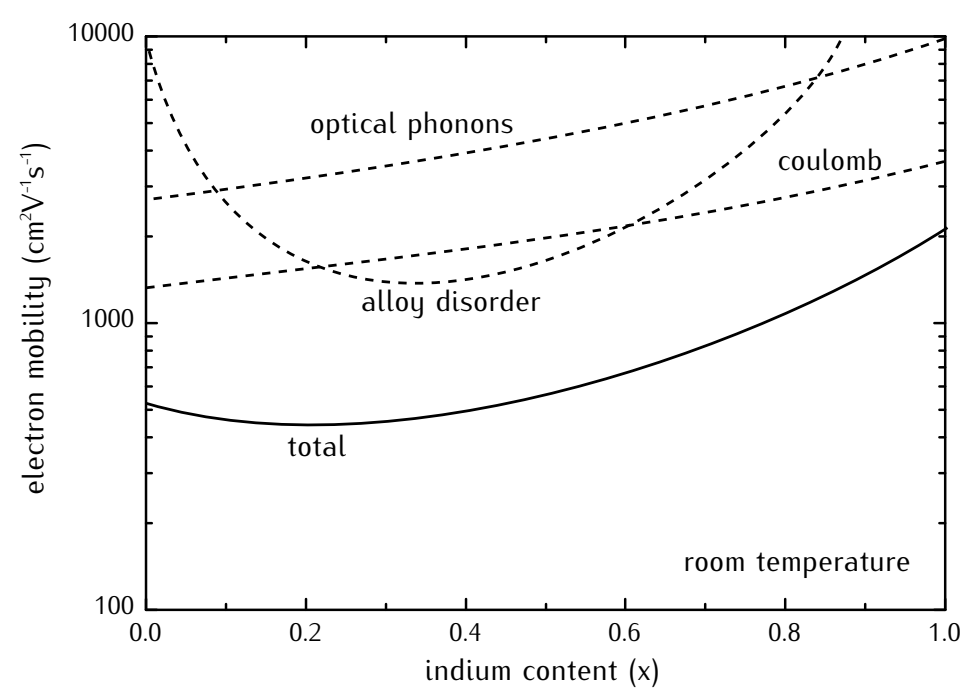

Figure 3.6: Calculated bulk electron mobility of InGaN at room temperature over In concentration [91].

of heterostructures is of great importance in order to achieve high electron mobilities at low temperature (see Section 5.2 and Section 6.4).

Different aspects of growth influence the stated scattering events. The quality of the interface to the substrate mainly determines the dislocation density. Scattering at ionized defects is a measure for the density of vacancies and other point defects. The interface roughness is an indicator for smooth and sharp interfaces. Only if all these parameters are optimized, high mobilities can be achieved, making $\mu$ a benchmark for the overall quality of grown heterostructures.

Scattering mechanisms affect the transport in AlGaN and InGaN based structures in a comparable way except for the alloy scattering. In GaN/AlGaN structures the 2DEG forms within the GaN and the quality of this layer largely determines the mobility. Electrons can be found in the AlGaN only with a low probability (Figure 3.5 a)). The impact of alloy scattering from disorder in AlGaN on the mobility in GaN/AIGaN heterostructures is thus small. In GaN/InGaN/GaN structures, the 2DEG forms within InGaN. Most of the electrons will therefore be affected by alloy scattering. However, the effective reduction of the mobility is only small as shown in Figure 3.6. Electrons in $\ln N$ have a lower effective mass than in GaN and AIN [90] and therefore a higher mobility. The mobility in bulk InGaN is therefore comparable to GaN for low indium contents with alloy scattering taken into account [91]. Electron mobilities in AlGaN based 2DEGs as high as $\mu=168000 \mathrm{~cm}^{2} \mathrm{~V}^{-1} \mathrm{~s}^{-1}$ at $0.5 \mathrm{~K}$ around $n_{s}=2 \times 10^{12} \mathrm{~cm}^{-2}$ have been achieved [46]. Such high values were achieved for RMS roughnesses well below $1 \mathrm{~nm}$ and low dislocation densities. Reproducible state of the art results on substrate with dislocation densities above $10^{8} \mathrm{~cm}^{-2}$ are however restricted to low-temperature mobilities between $\mu=20000 \mathrm{~cm}^{2} V^{-1} \mathrm{~s}^{-1}$ and $\mu=50000 \mathrm{~cm}^{2} V^{-1} \mathrm{~s}^{-1}$ for otherwise 
similar crystal quality [92]. At room temperature 2DEG mobilities as high as $\mu=2500 \mathrm{~cm}^{2} \mathrm{~V}^{-1} \mathrm{~s}^{-1}$ have been measured [45] which is twice as much as the highest bulk mobility of GaN reported [93].

Over the past years, the $\ln N$ bulk mobilities at room temperature have increased up to $\mu=3500 \mathrm{~cm}^{2} \mathrm{~V}^{-1} \mathrm{~s}^{-1}$ [94]. No reports on high electron mobilities at low-temperature for 2DEGs with InGaN layer grown by MBE were found. This indicates the far more challenging growth of InGaN compared to AIGaN. For MOVPE grown heterostructures with InGaN channels, the highest reported mobilities are just above $\mu=1000 \mathrm{~cm}^{2} \mathrm{~V}^{-1} \mathrm{~s}^{-1}$ at $4 \mathrm{~K}$ [95].

\subsection{HALL EFFECT: A BRIEF REMINDER}

The previous sections explained the existence of a $2 \mathrm{D}$ carrier confinement in III-nitride heterostructures with the sheet density $n_{s}$. The dependence of the mobility $\mu$ on different scattering mechanisms was presented. Both $\mu$ and $n_{s}$ can be obtained by measurements of the Hall effect. This section recalls the effect in brief and states the measurement geometries used in this work. Electrons have the charge $-e$ and are accelerated by an electric field $\vec{E}$. After a certain time $\tau_{t r}$, the electrons scatter which results in an average velocity called drift velocity $\overrightarrow{v_{d}}$ :

$$
\overrightarrow{v_{d}}=-\frac{e \tau_{t r} \vec{E}}{m^{*}}=\mu \vec{E}
$$

here $m^{*}$ is the effective mass.

The mobility $\mu$ thus indicates how the drift velocity of electrons scales with an electric field. With the current density $\vec{j}=-$ e $n \overrightarrow{v_{d}}=\sigma \vec{E}$, the basic description of the conductivity $\sigma$ depending on the carrier density $n$ and $\mu$ is obtained:

$$
\sigma=e n \mu
$$

The conductivity $\sigma$ can be calculated directly from resistance measurements for a known sample geometry. In order to gain the mobility $\mu$, the carrier density has to be known. This parameter is obtained from Hall effect measurements.

If a magnetic field $B$ is applied to an electrical current $I$, carriers are deflected by the Lorentz force (Figure 3.7). This results in charge depletion on one and accumulation on the other site of the material. The resulting electric field is opposite to the direction of the Lorentz force. In equilibrium, the Lorentz force and the force by the electric field compensate each other. The charge separation is measurable by the Hall voltage $U_{H}$ :

$$
w U_{H}=R_{H} B I
$$

here, $w$ is the width of the sample. The Hall constant $R_{H}$ depends only on number and charge of the carriers: $R_{H}=-\frac{1}{e n}$. The electron concentration can thus be calculated from the lateral resistance under perpendicular 


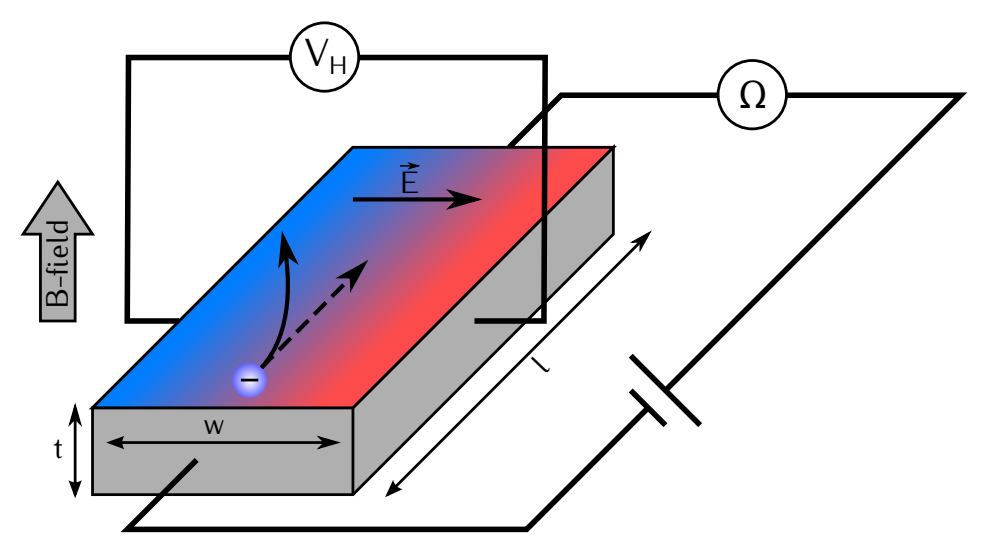

Figure 3.7: Schematic of the Hall effect in a sample with width $w$, length $l$ and thickness $t$. Electrons are deflected in a magnetic field $B$ by the Lorentz force. This results in a charge accumulation which is indicated by the color code. The resulting electric field $\vec{E}$ compensates the Lorentz force in equilibrium. The potential difference by the charge separation is measured by the Hall voltage $V_{H}$.

applied magnetic fields. The electron mobility is then calculated from the longitudinal resistance and the carrier concentration obtained from the Hall measurements.

A heterostructure will have more than one contribution to the conductance. The example in Figure 3.8 considers a GaN/InGaN/GaN with carriers in bulk GaN, a 2DEG and a 2DHG. Each of the different carriers will contribute a resistance to a parallel circuit. A reliable measurement of, e.g. the 2DEG is thus only possible if $\sigma_{2 D E G} \gg \sigma_{2 D H G}$ and $\sigma_{2 D E G} \gg \sigma_{b u l k}$. If the mobilities of the confined carriers are comparable, this implies that sheet electron density $n_{S E}$ of the 2DEG is large compared to the sheet hole density $n_{S H}$ of the 2DHG. This can be achieved by an appropriate heterostructure design (see Section 6.4). To minimize the contribution of the bulk $n_{S E} \gg n_{\text {bulk }}$ or $\mu_{2 D E G} \gg \mu_{\text {bulk }}$. This is not true at high temperatures where the mobility of the bulk is comparable to the 2DEG. Reliable magnetotransport measurements of the 2DEG are thus only possible at low temperatures.

The Hall measurements in this work were performed for two different geometries. The van der Pauw geometry [96] was used for quick analysis. Here, the native oxide is damaged mechanically in the corners of a $3 \times 3 \mathrm{~mm}^{2}$ sample and four indium contacts are pressed on the surface. Because of the large measured area, the impact of inhomogeneities is large and can lead to inaccurate results. The Hall bar geometry measures a much smaller area. However, the preparation is more extensive and was used for detailed measurements of promising samples.

The Hall bar structures, with length $l=520 \mu \mathrm{m}$ and width $w=170 \mu \mathrm{m}$, were produced by lithography. The contacts consisted of $20 \mathrm{~nm}$ titanium, $40 \mathrm{~nm}$ aluminium, $25 \mathrm{~nm}$ titanium and $40 \mathrm{~nm}$ gold. They were annealed at $850{ }^{\circ} \mathrm{C}$ 


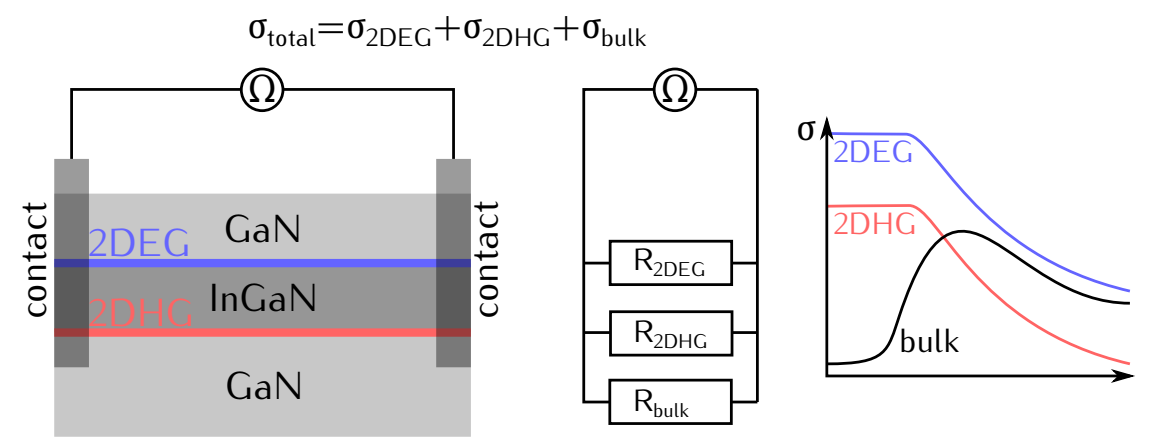

Figure 3.8: Electrical measurement of parallel channels for the example of a GaN/InGaN/GaN heterostructure. Three channels are present: 2DEG, 2DHG and bulk GaN. Each channel represents a resistance in a parallel circuit. The conductances of the channels add up and a reliable measurement of one channel is only possible if it more conductive than the others. Since the temperature dependence of $\sigma$ is different for 2D and 3D transport, reliable $2 \mathrm{D}$ measurements are best performed at low temperatures.

under nitrogen atmosphere for $30 \mathrm{~s}$. The background and optimization of the metalization can be found in the literature[97, 98, 99]. In order to measure only a well defined area, the structures were etched outside of the Hall bar by reactive ion etching.

\subsection{MAGNETORESISTANCE}

Various scattering mechanisms determine the electron mobility. Therefore, $\mu$ is a measure for the overall sample quality. To identify the dominating scattering mechanisms, the magnetoresistance was studied in this work. Magnetoresistance means the magnetic field dependence of the longitudinal resistance $R_{x x}(B)$. The classical Drude theory predicts no change of the resistance with magnetic field [100]. The observation of magnetoresistance phenomena is thus evidence of more physically rich effects. During this work, the well known non classical effects of weak localization (WL) and Shubnikovde Haas oscillations (SDH) were observed. For a complete description of the magnetoresistance, the classical influence of structural effects has to be taken into account as well.

The following subsections very briefly describe the different observed sources of magnetoresistance and state the information they provide. More detailed explanations of SDH oscillations and WL are found in standard textbooks $[100,101,102]$. At the end of the section, a graphical summary of different contributions to the magnetoresistance is given, adding the effects of weak antilocalization and electron-electron interaction which were not studied in this work.

It should be noted that all effects are present in every sample. However, their observation depends on temperature, sample quality and magnetic field. In 
magnetic fields, electrons are forced into a circular motion with the cyclotron frequency $\omega_{c}$. The condition $\omega_{c} \tau_{t r}=\mu B_{t r}>1$ means that the electrons can complete at least one cyclotron orbit before they scatter. For magnetic fields smaller than $B_{t r}$, the transport is diffusive and weak localization is observed. At $B>B_{t r}, \mathrm{SDH}$ oscillations are possible. Both effects require a high mean free path and are therefore only observed at low temperatures.

\subsubsection{Shubnikov-de Haas oscillations}

Shubnikov-de Haas (SDH) oscillations are observed for transport at low temperatures for high magnetic fields [100]. The example in Figure 3.9 shows the oscillations of the longitudinal resistance with increasing magnetic field. In the following this effect is only introduced in brief in order to illustrate the information that is gained from this magnetoresistance effect. A more detailed description of underlying mechanisms and the stated formulas is found in the literature $[100,101]$.

The energy eigenvalues of a two dimensional system confined in z-direction are:

$$
E_{j}=\frac{h^{2}}{8 \pi^{2} m_{x} *} k_{x}^{2}+\frac{h^{2}}{8 \pi^{2} m_{y}{ }^{*}} k_{y}{ }^{2}+\epsilon_{j}
$$

The confinement to two directions thus leads to a quantization of states as shown in Figure 3.10. The index j numbers different subbands.

Carriers will move in a circular motion with the cyclotron frequency $\omega_{c}=\frac{e B}{m^{*}}$, if a magnetic field is applied perpendicular to the confined plane. Only discrete kinetic energies are allowed for a quantum mechanical description of this motion, which results in the formation of Landau levels (LL) as shown

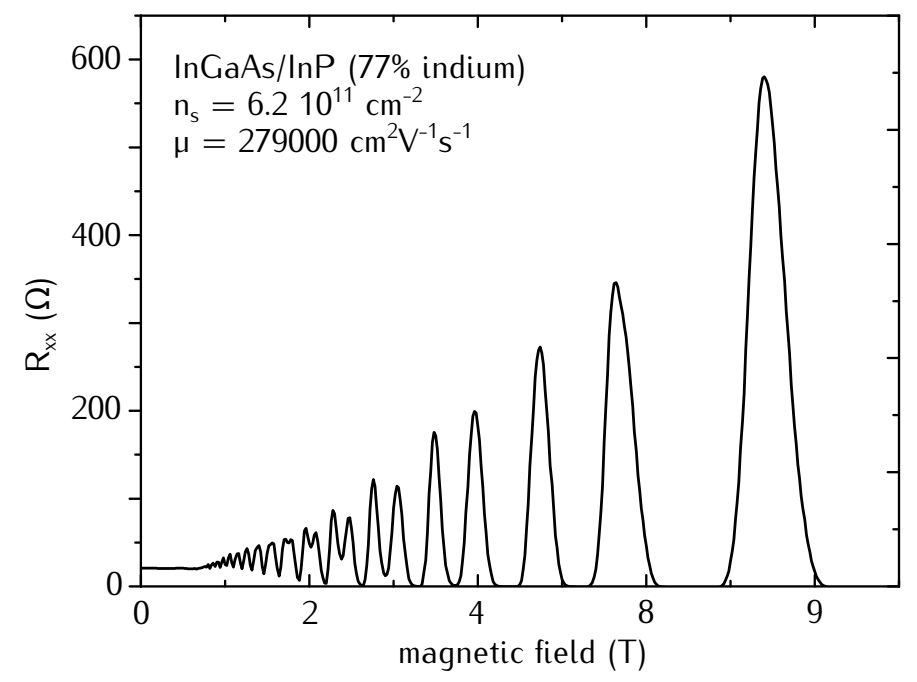

Figure 3.9: Example of $R_{x x}(B)$ showing Shubnikov-de Haas oscillations [103]. 
Figure 3.10

Energy over density of states for a two dimensional system with zero magnetic field and large magnetic field applied. Separated Landau levels form for applied $B$-field.
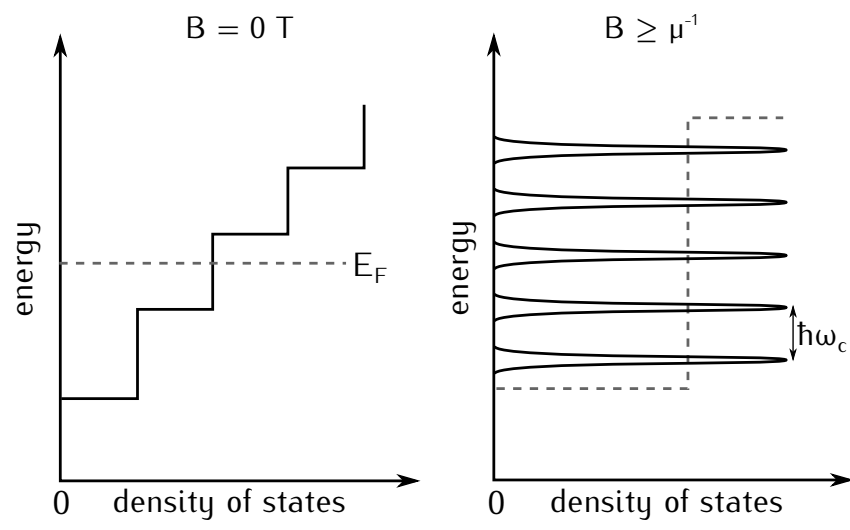

in Figure 3.10. The energy of the $n$-th landau level for a subband $\mathrm{j}$ may be written as:

$$
E_{j, n}=\epsilon_{j}+\frac{h}{2 \pi}\left(n+\frac{1}{2}\right) \omega_{c}
$$

if Zeemann splitting is not taken into account. The number of states per LL is given by:

$$
N_{L L}=\frac{e B}{h} g_{s}
$$

with the spin degeneracy $g_{s}$.

The oscillating nature of the magnetoresistance is explained by the magnetic field dependence of the LLs. The energy difference between the LLs increases with magnetic field. During this process, different LLs will pass the Fermi energy. Each time the Fermi energy is at the center of a $L L$ there is a change in the resistivity and $\rho_{x x}$ oscillates for varying magnetic field. This allows to calculate the carrier density $n_{s}$.

If the magnetic field changes, so does the number of occupied LLs. The resistivity is maximal each time the Fermi level lies at the center of a LL and the number of occupied Landau levels is a half-integer. The relation of the magnetic fields $B_{1}$ and $B_{2}$ of two successive peaks is thus:

$$
\frac{h n_{s}}{2 e B_{1}}-\frac{h n_{s}}{2 e B_{2}}=1
$$

The number of carriers can thus be calculated from the position of two or more peaks in the resistivity. This method is more accurate than the extraction from the classical Hall resistance.

The Landau levels alone may explain the oscillating nature of $\rho_{x x}(B)$ but cannot explain the amplitude and especially not $\rho_{x x}=0$. One way to illustrate this behavior is the presence of edge channels.

Every measured structure will be finite. Sample boundaries can be seen as potential barriers. At the edge of a sample, this leads to a rise of the LL energy as shown in Figure 3.11 a). The Fermi energy will thus always cross 
a landau level at the edges. Electrons at the edges thus always contribute to conduction. Under a magnetic field, these edge states will exhibit a specific electron motion.

The electrons within the sample are moving in a circular motion. At the very edge, this is not possible. Here, carriers are drawn along the edge as shown in Figure 3.11. Scattering processes do not change this motion, as the electron will be drawn in the same direction as before the scattering event. For sufficient high fields, this results in zero resistance.

Each time a LL crosses the Fermi level within the sample, states between the edges states become available. Electrons from the edge channels can now be scattered into states at the opposing edge. This backscattering results in an increased resistivity.

Potential fluctuations result in a broadening of the LLs. This can be seen as the influence of scattering potentials that limit the lifetime of an electron in a certain $L L$, i.e. quantum state. This lifetime is called quantum lifetime $\tau_{q}$ and has an impact on the amplitude of the SDH oscillation [104]:

$$
\frac{\Delta \rho_{X X}(B)}{\rho_{X X}(B=0)}=1-2 \exp \left(-\frac{\pi m^{*}}{\tau_{q} \text { e } B}\right)\left(\frac{\chi}{\sinh \chi}\right) \cos \left(2 \pi E_{F}\right)
$$

with $\chi=\frac{4 \pi^{3} k_{b} m^{*} T}{e h B}$.

The implicit temperature dependence is only in $\chi$. The effective mass $m^{*}$ can thus be calculated by comparing the oscillations at different temperatures [105]. For a known effective mass, the quantum lifetime $\tau_{q}$ is the only unknown variable left and can be obtained by fitting the experimental data [105].

The ratio of transport lifetime $\tau_{t r}$ and quantum lifetime $\tau_{q}$ provides information about the dominant elastic scattering process. The transport lifetime is derived from the mobility which is determined from electrons moving along an applied field in one direction. Scattering processes with small angles will
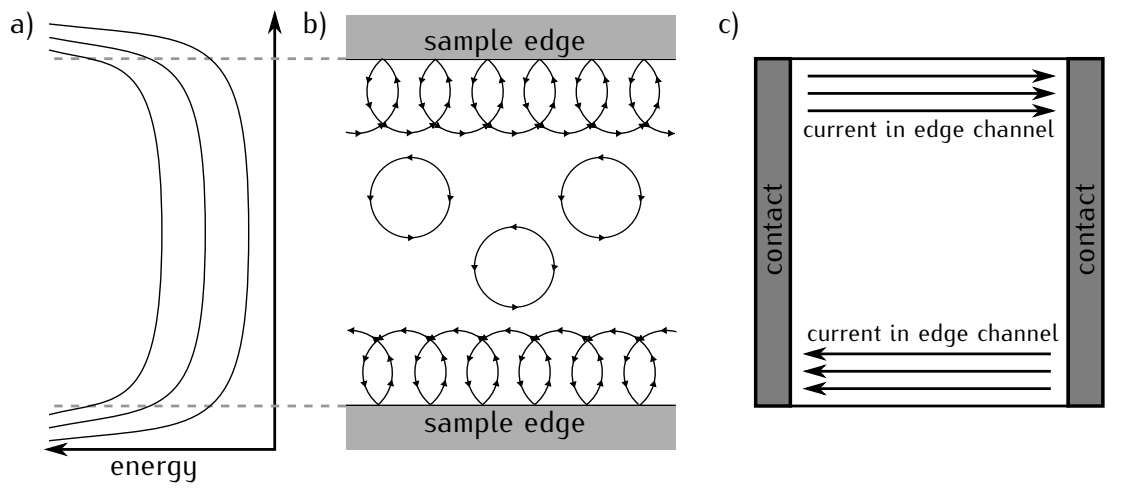

Figure 3.11: Edge channel model: a) energetic position of Landau levels over sample width with an upward band bending at the edges. b) electron motion in the sample with cyclotron motion in the center and drift at the edges due to magnetic field. c) channels at the edges carrying the current. 
Figure 3.12

Schematic of electron propagating through a medium with a) large angle scattering and b) small angle scattering. $\tau_{q}$, which accounts for every scattering event equally, is similar in a) and b). $\tau_{t r}$ increases for large angle scattering in b) compared to a).

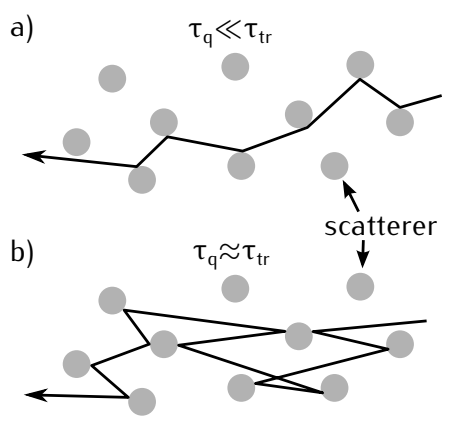

therefore have less impact on $\tau_{t r}$ than large angle scattering events as is depicted in Figure 3.12. The quantum lifetime accounts for every scattering event equally. The ratio of $\tau_{t r} / \tau_{q}$ is thus a measure of the scattering angle of the dominating scattering process $[106,107]$.

\subsubsection{Weak localization}

Weak localization can be observed for diffusive transport [108, 109]. In this regime, electrons are scattered along their way. Not every scattering process is inelastic and the phase information is not lost at every scattering event. Therefore, two time scales are relevant: the elastic scattering time $\tau_{e}$ and the inelastic scattering time $\tau_{i}$. At higher temperatures inelastic electron-phonon scattering dominates the carrier transport. Only for lower temperatures $\tau_{i}$ can be significantly larger than $\tau_{e}$. Under these conditions, weak localization is observed.

There are many possible paths an electron may follow while moving through a medium with elastic scatters as illustrate in Figure 3.13. During propagation from $A$ to $B$, an electron will change its phase. The phase is changed reversibly and not lost for elastic scattering. The probability of any electron being transported from A to B depends on the way taken and is also influenced by interference. For most possible trajectories, interference will be unlikely because of the random distribution of phases.

The situation changes for electron trajectories that cross each other. In such a loop, an electron can take two different directions, ending at the same

Figure 3.13

Possible path of an electron from $A$ to $B$ in a medium with scatters. The motion along closed loops is possible in two directions. Electrons in closed loops do not contribute to current and which results in weak localization.

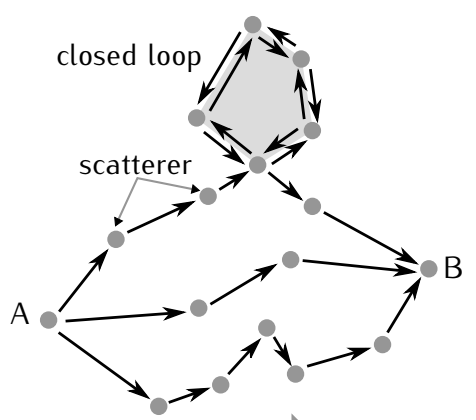

current 
point after the same amount of scattering events with an identical phase. It can be shown, that the probability of carriers propagating on closed loops is increased, if interference is taken into account [100]. This implies that the resistance will increase compared to the classical transport as electrons are localized in the closed loops and do not contribute to a current. The maximum size of a closed loop will depend on the inelastic scattering time because the phase needs to be conserved for constructive interference. WL is therefore only observed if $\tau_{e}<\tau_{i}$.

The previous consideration implied no magnetic field. In the presence of a magnetic field, the direction in the loop will influence the trajectory and the phase. At zero field, loops of all sizes contributed to a change in resistivity. For increasing magnetic field, the size and thus the number of closed loops decreases. The resistance therefore decreases with increasing field and the classical value is obtained at high fields. A resulting magnetoresistance measurement is shown in Figure 3.14.

In high quality samples, the scattering centers are further apart than in structures of lower quality. The smallest possible loops therefore cover a large area in high mobility samples. As large closed loops are already prohibited at low magnetic fields, the $W L$ is observed only at low $B$-fields if $\mu$ is large. With increasing temperature, the inelastic scattering length decreases rapidly. If the phase information is lost within a closed loop due to inelastic scattering, this loop will not contribute to WL. WL is thus only observed at low temperatures and vanishes if every scattering event is inelastic.

The correction of the conductivity by WL can be written as:

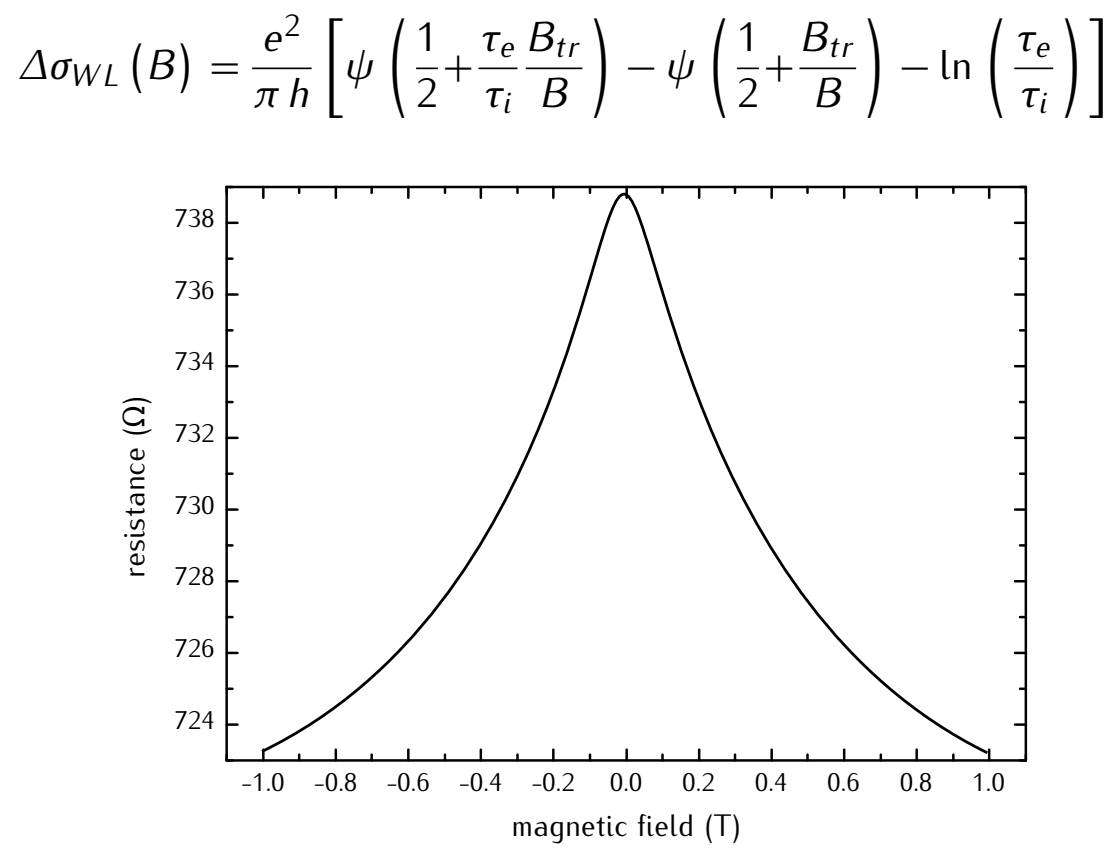

Figure 3.14: Example of $R_{x x}(B)$ with weak localization measured at $\mathrm{T}=130 \mathrm{mK}$ in delta-doped silicon [110]. 

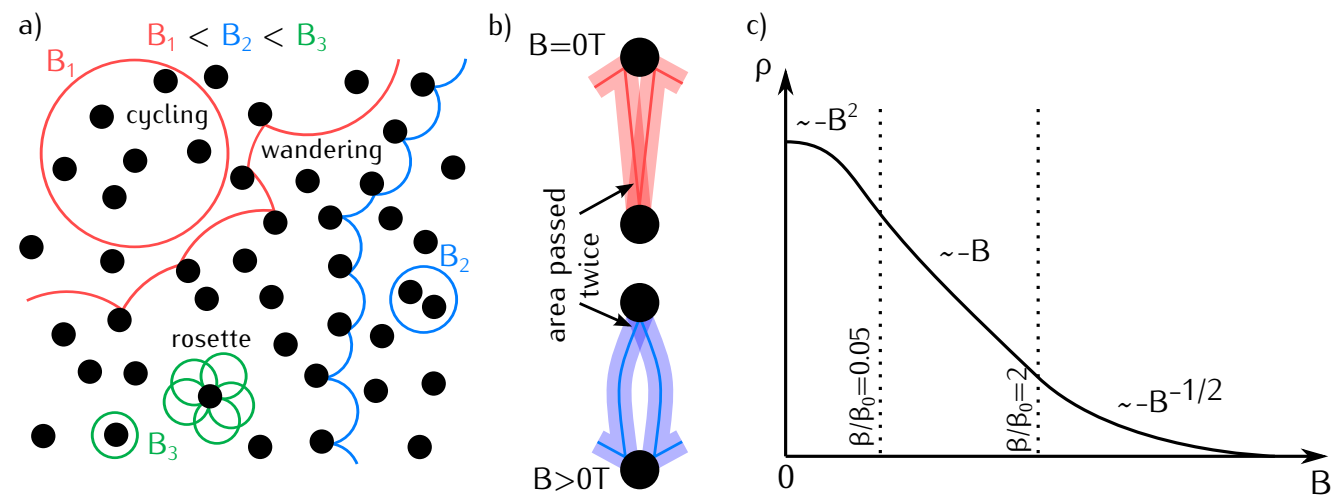

Figure 3.15: Transport influenced by a potential of impenetrable discs. a) Trajectories of carriers at different magnetic fields. Cycling carriers describe circular motions and do not contribute to current. At large fields, carriers are scattered at the same disc multiple times, which results in a rosette like motion. The area covered by the rosette is larger compared carriers just moving in cyclotron trajectories and are therefore more likely to scatter. b) Illustration of the empty corridor. Due to backscattering an area is passed twice by a carrier, which is free of scatterers. This area decreases with increasing magnetic field. c) The resistivity resulting from this model exhibits three different $\rho_{x x}(B)$ dependencies, observable at low, intermediate and high $B$-fields.

here $\tau_{e}$ is the elastic scattering time, $\tau_{i}$ is the inelastic scattering time, $\psi(x)$ is the digamma function and $B_{t r}=\frac{h}{4 \pi^{2} e l_{e}^{2}}$ is the transport magnetic field with the elastic mean free path $l_{e}$. By fitting the experimental conductivity the elastic mean free path $l_{e}$, as well as the ratio $\tau_{e} / \tau_{i}$, are obtained. From the temperature dependence of $\tau_{i}$, the dimension of transport, as well as the dominant inelastic scattering process, can be gained $[111,112]$.

\subsubsection{Classical influence of scattering potentials}

The Drude model predicts no magnetic field dependence of the longitudinal resistance $R_{x x}$. The Lorentz model gives a more physically rich description of transport in a medium. It investigates non interacting, free electrons scattered at randomly distributed impenetrable disc potentials of diameter $a$ and a mean separation $l_{a}$ [113]. This model predicts a change of $R_{x x}$ with magnetic field.

Possible electron trajectories in a random disc array for different magnetic fields are depicted in Figure 3.15 a). At low magnetic field, the cyclotron radius of the electrons is large. Only few electrons describe a circular motion without being scattered. They are called cycling electrons. Many wandering electrons are scattered and described a diffusive motion. Because cycling electrons do not move through the medium they do not contribute to carrier transport. With increasing magnetic field their number increases. This leads 
to a decreased because the carriers follow circles with smaller radius and scattering is less likely.

The cycling electrons do influence the longitudinal conductivity $\sigma_{x x}$ as well as the Hall conductivity $\sigma_{x y}$. To find the correct expression for the longitudinal magnetoresistance, the $\rho_{x x}$ element of the resistivity tensor has to be calculated. The result is a negative exponential magnetoresistance.

This result is only valid in the limit of point scatterers. Finite size discs allow for recollision processes, especially at higher magnetic fields. The electrons then follow a rosette like trajectory which covers a larger area compared to the cyclotron orbit as depicted in Figure 3.15 a). This leads to an increased probability of scattering at other discs and modifies the exponential behavior of $R_{x x}$ [114].

At lower magnetic field, the empty corridor effect has to be considered which is illustrated in Figure 3.15 b) [115]. After backscattering, an electron might pass through the same region as it traveled before scattering. There is no scatterer in this corridor and the probability of scattering is reduced. Because the electron moves in the opposite direction after backscattering, it is deflected in a different direction by a magnetic field. The probability of following an empty corridor is therefore reduced at higher magnetic fields. Numerical calculations taking both considerations into account result in the following magnetoresistance [116]:

$$
\frac{\rho-\rho_{0}}{\rho_{0}}=\beta_{0} \begin{cases}0.32 \frac{\beta}{\beta_{0}} 2 & \text { for } 0 \leq \frac{\beta}{\beta_{0}}<0.05 \\ 0.032 \frac{\beta}{\beta_{0}}-0.04 & \text { for } 0.05 \leq \frac{\beta}{\beta_{0}} \leq 2 \\ 0.39-1.3 \frac{\beta}{\beta_{0}}-0.5 & \text { for } \frac{\beta}{\beta_{0}}>2\end{cases}
$$

with $\beta=\omega_{c} \tau$ and $\beta_{0}=\frac{a}{l_{a}}$, where $a$ is the disc radius, $\omega_{c}$ the cyclotron frequency, $\tau=\frac{l}{v_{f}}$ the mean free time and $l$ the mean free path. Three

a)

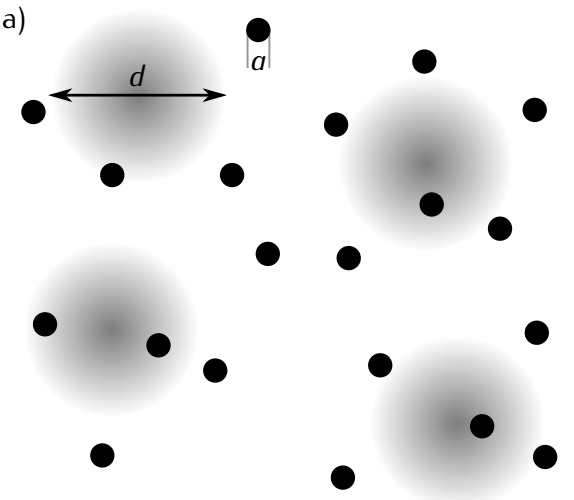

b)

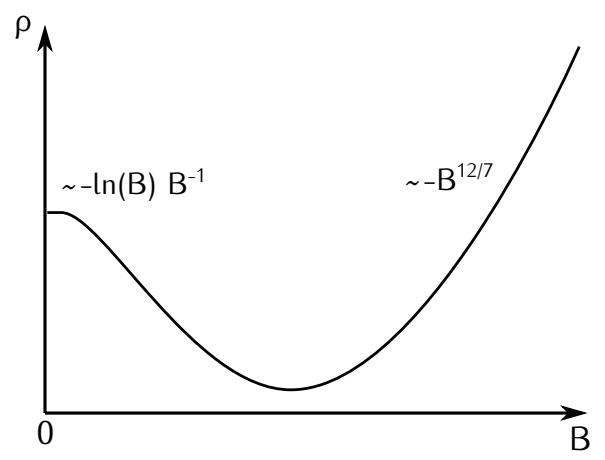

Figure 3.16: Transport influenced by a potential of impenetrable discs and a smooth random potential. a) illustration of the potential, b) the characteristic magnetoresistance influenced by such a potential is negative at low $B$-fields and positive at higher $B$-fields. 
different dependencies of $R_{x x}$ on the magnetic field are found. The ratio of $a / l_{a}$ can be obtained from the values of the magnetic field at the transitions. The magnetoresistance changes drastically if a smooth potential is added to the random array of impenetrable discs as illustrated in Figure 3.16 a). The potential is characterized by smooth changes in the potential landscape over a diameter $d$ separated by the distance $l_{d}$ with $d \gg a$ and $l_{d} \gg l_{a}$. Depending on the four parameters $a, l_{a}, d$ and $l_{d}$ various dependencies of $R_{x x}$ on the magnetic field are expected [114]. One example is given in Figure $3.16 \mathrm{~b})$. 


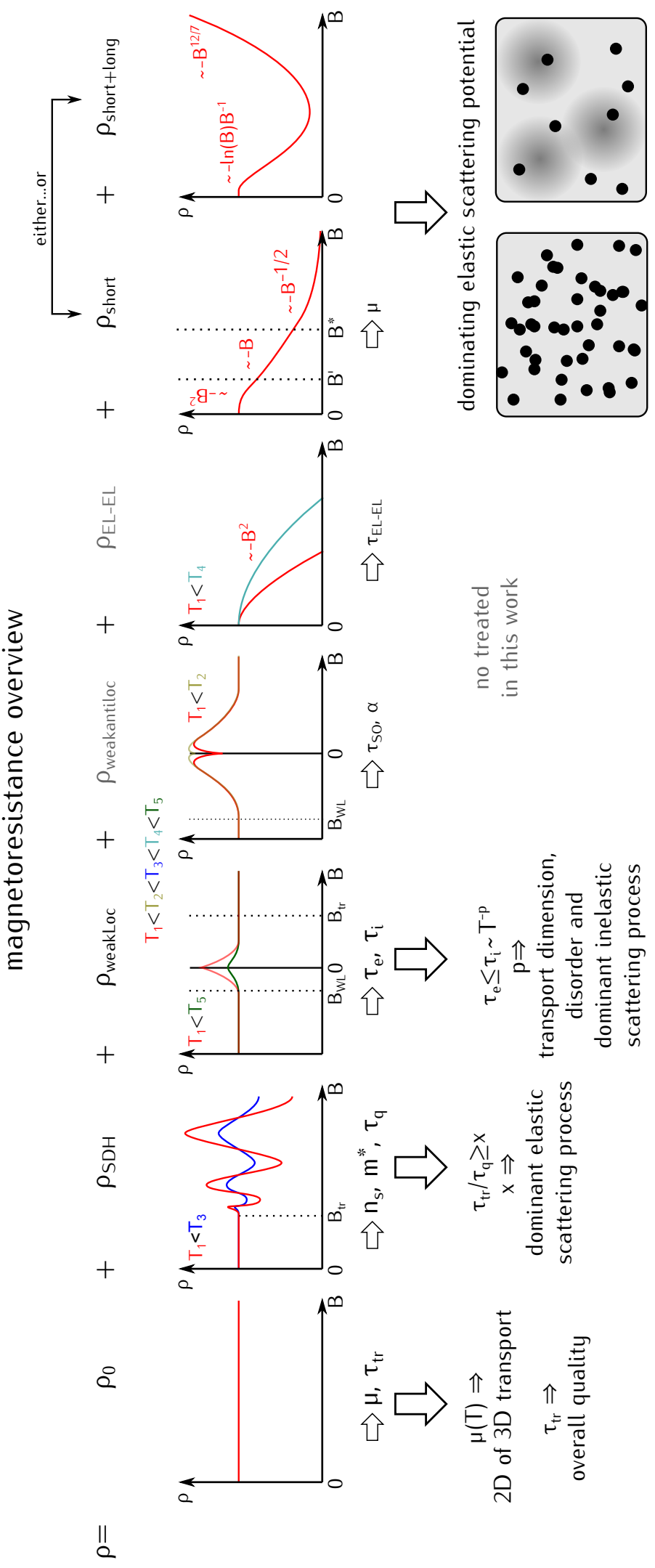

Figure 3.17: Overview of different contributions to the magnetoresistance together with the parameters than can be obtained from $R_{x x}(B)$. WAL and El-El have not been studied in this work and are given for completeness. 

High quality polar GaN, AlGaN and InGaN layers grow under metal bilayer stabilized conditions by MBE. The typical nitrogen pressure in the growth chamber is $p_{\text {growth }}=10^{-5}$ mbar, which is low compared to VPE techniques. At this pressure, GaN decomposes at $800{ }^{\circ} \mathrm{C}$ for $\mathrm{N}$-rich or stoichiometric conditions. This temperature is too low for the activation of kink sites, which is needed for smooth 2D growth modes. Growth under gallium or indium bilayer stabilized conditions increases the surface diffusion of nitrogen compared to diffusion on the bare GaN (0001) surface. In addition, the metallic adlayers reduce the decomposition temperature to $720^{\circ} \mathrm{C}$, which decreases the kinetic barrier of kink sites. Two dimensional spiral growth is observed under bilayer conditions and atomically flat morphologies are obtained. The growth on affordable foreign substrates by MBE results in a higher dislocation density than growth by other techniques. Heterostructures with low dislocation densities are therefore grown on templates.

RHEED is a versatile in-situ technique in MBE. The RHEED patterns provide information about the roughness, the orientation and the in-plane lattice constant of the growing film. The RHEED intensity can be used to analyze the growth rate, the III/V ratio and the metal coverage. It is thus a powerful tool for growth optimization.

Group III-nitrides exhibit strong pyroelectric polarization. This enables two dimensional carrier confinement in heterostructures without modulated doping. Polarization fields cause electrons to accumulate within GaN in GaN/Al$\mathrm{GaN}$, while holes accumulate in InGaN in GaN/InGaN heterostructures. The low-temperature mobilities of the confined carriers depend strongly on the interface quality and on the bulk crystal quality of GaN for GaN/AIGaN and of InGaN for GaN/InGaN heterostructures. The analysis of the magnetoresistance at low temperatures provides information about the dominant scattering mechanisms. The low-temperature electrical characterization can thus be used to examine the impact of different growth optimization steps on the quality of grown heterostructures.

In the next part, the growth optimization process of GaN, GaN/AlGaN and GaN/InGaN structures will be discussed in detail. The starting point of optimization will be based on the considerations and publications presented in part I. RHEED is used in a great part of the process. The impact of each optimization step on structural and low-temperature electrical properties will be presented. 

Part II

RESULTS OF GROWTH OPTIMIZATION 



\section{GALLIUM NITRIDE}

GaN layers grown in the [0001] direction are the basis for all GaN/AlGaN and GaN/InGaN structures investigated in this work. The optimization of GaN growth is therefore a key factor for the quality of heterostructures. This chapter presents the optimization process and the parameters obtained for high quality GaN growth in detail. For each step, the effects on the structural quality are presented. Low-temperature 2DEG mobilities of GaN/AlGaN structures, which are highly dependent on the GaN quality, complement the results.

\subsection{SUBSTRATE PRETREATMENT}

The lack of suitable substrates is a challenge in GaN growth. Sapphire is the material of choice in most cases. In order to obtain a good crystal quality, various growth steps and thick layers are required [36, 12]. Great progress has been made, reducing the density of threading dislocation for GaN (0001) grown by MBE directly on sapphire significantly [18]. Nevertheless, the typical number of dislocations is higher than for commercially available templates. These substrates consist of thick GaN layers grown directly on sapphire by other techniques. The structures presented in this work were grown on MOVPE grown GaN templates consisting of $3 \mu \mathrm{m}$ GaN on sapphire [16] with a nominal dislocation density of $4 \times 10^{8} \mathrm{~cm}^{-2}$.

The first optimization step thus addresses the MOVPE/MBE interface. Contaminations at this interface are the source of additional dislocations. During optimized growth by MBE the number of dislocations does not reduce significantly. Dislocations follow the growth direction and do not annihilate or leave the crystal at its boundaries. The dislocation density is thus mainly determined before the actual growth and the dislocation density of the template determines the smallest possible number of dislocations for a grown structure. This stresses the importance of the pretreatment of the 

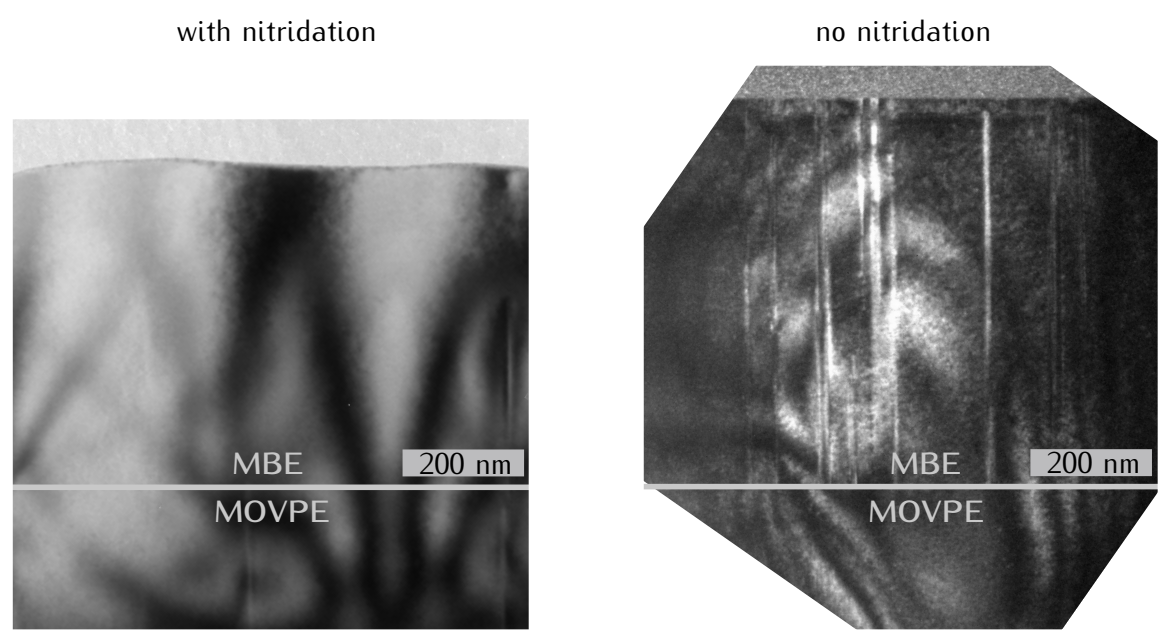

Figure 4.1: Transmission electron micrographs of a sample with nitridation (Sample ID: G0166) and a sample without nitridation (Sample ID: G0107) before GaN growth. Dislocations form at the MOVPE/MBE interface without nitridation.

substrate.

All structures were grown on $10 \times 10 \mathrm{~mm}^{2}$ pieces which were cut from 2 -inch wafers. After dicing, the substrates were cleaned first with acetone and then with 2-propanol in an ultrasonic bath at low power. They were then blown dry with nitrogen quickly to prevent stains from drying solvents. Afterwards, the samples were heated up to $200{ }^{\circ} \mathrm{C}$ in the loadlock and up to $600{ }^{\circ} \mathrm{C}$ in the transfer vacuum chamber for $10 \mathrm{~min}$ respectively. This time was sufficient for a decrease of the background pressure while longer baking times resulted in carbon formation as indicated by Auger electron spectroscopy. The template surface did not show any contaminations after this cleaning process in atomic force micrographs.

However, residual impurities of carbon, oxygen and silicon absorbed on the surface cannot be detected by AFM. Such contaminations induce a high number of threading dislocations [93]. By treating the substrate with activated nitrogen for $15 \mathrm{~min}$, no additional dislocations formed at the MOVPE/MBE interface as shown in Figure 4.1. Consequently, this step significantly improved the 2DEG mobility of GaN/AIGaN structures. For a sample with nitridation, the mobility was $\mu=5200 \mathrm{~cm}^{2} \mathrm{~V}^{-1} \mathrm{~s}^{-1}$ with $n_{s}=7.2 \times 10^{12} \mathrm{~cm}^{-2}$ at $4 \mathrm{~K}$ while for an otherwise identical sample without this step the mobility was only $\mu=1918 \mathrm{~cm}^{2} \mathrm{~V}^{-1} \mathrm{~s}^{-1}$ with $n_{s}=6.8 \times 10^{12} \mathrm{~cm}^{-2}$ at $4 \mathrm{~K}$.

\subsection{OPTIMUM GROWTH CONDITIONS}

Growth conditions in MBE are given by the substrate temperature, the nitrogen flux and the metal flux. Gallium bilayer stabilized conditions produce the best results for GaN (0001) in terms of crystal quality and surface 
morphology (see Section 1.4 and Section 1.5.2). Growth in this regime requires a sufficient Ga desorption rate to avoid droplet formation. Adequate high substrate temperatures are therefore needed depending on the applied nitrogen conditions. The gallium flux is then chosen according to the Ga coverage to provide a stable Ga bilayer. At higher temperatures, the GaN decomposition rate becomes comparable to the growth rate and limits the applicable substrate temperatures.

The nitrogen conditions are given by the power supplied to the high frequency alternating field of the plasma source $P$ and the flow rate of molecular nitrogen $F_{N 2}$. Depending on the two parameters different ratios between activated molecular and atomic nitrogen species are obtained as shown in Figure 4.2. No stable plasma is achieved for high nitrogen fluxes at low excitation powers. At higher powers, the amount of atomic nitrogen increases compared to molecular nitrogen species. As atomic nitrogen mainly contributes to growth [117], the growth rate increases not only with the nitrogen flow but also with the plasma power. It has however been reported that higher plasma powers generate a higher density of point defects [44]. All samples presented in this work were grown with a power of $300 \mathrm{~W}$ and a flow rate of $0.5 \mathrm{sccm}$. The resulting growth rate is $3 \mathrm{~nm} / \mathrm{min}$ for a Ga-flux corresponding to stoichiometry. The ratio of molecular to atomic nitrogen was 1.5. These conditions were chosen to allow for stable plasma operation at lower powers with a decent growth rate.

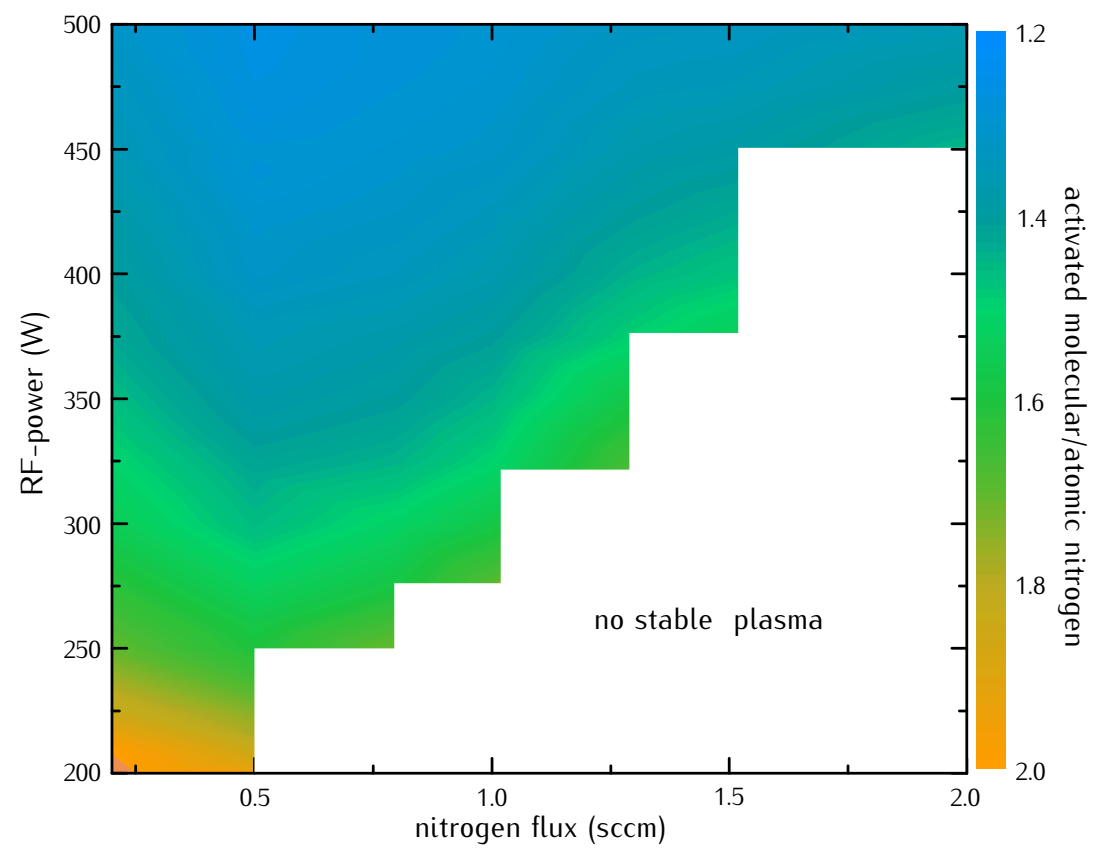

Figure 4.2: Ratio of activated molecular to atomic nitrogen for stable plasma conditions. The values were determined from the optical spectrum of the plasma by the ratio of two intensity ranges which are characteristic for atomic and molecular nitrogen transitions $[118,119]$. 


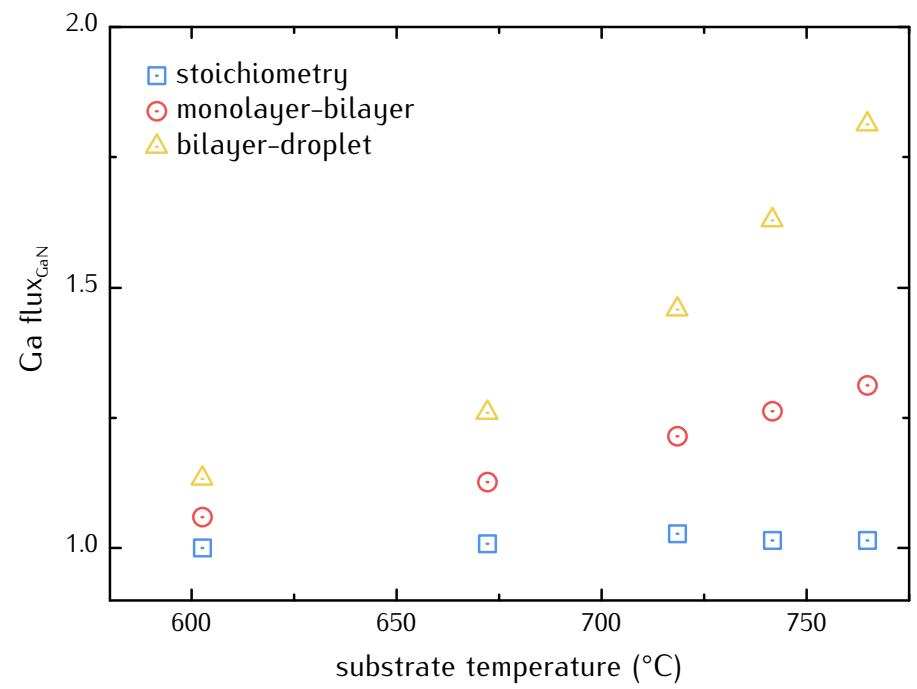

Figure 4.3: GaN bilayer diagram over substrate temperature and Ga-flux given normalized to the GaN stoichiometry at $600{ }^{\circ} \mathrm{C}$. Nitrogen conditions: $F_{N 2}=0.5 \mathrm{sccm}$ and $P=300 \mathrm{~W}$. The three transitions of the Ga coverage were determined by RHEED: N-rich to Ga-rich (stoichiometry), monolayer-bilayer and bilayer-droplet.

The gallium coverage was investigated by monitoring the RHEED intensity during gallium desorption. Here, the stoichiometry and the limits of stable bilayer coverage depending on the Ga-flux were obtained according to the methods introduced in Section 2.2.3 and Section 2.2.4. The gallium flux is given normalized to the stoichiometric flux. The results of this analysis at different temperatures are given in Figure 4.3. The stoichiometric point is constant within the investigated temperature range. The monolayer-bilayer and bilayer-droplet transitions increase with the substrate temperature. This is due to the enhanced desorption of Ga from the surface with increasing temperature. As a consequence, the bilayer regime vanished at temperatures below $T_{s}=600{ }^{\circ} \mathrm{C}$ and droplets form for fluxes higher than the stoichiometry. This marks the low temperature limit for growth under Ga bilayer stabilized conditions.

All samples grown under Ga bilayer stabilized conditions showed the spiral growth mode as depicted in Figure 4.4. This growth mode produces good surface morphologies with atomically flat terraces. For a more detailed study of the impact of the Ga/N ratio within the bilayer regime on the crystal quality, GaN/AIGaN 2DEG structures were grown for $1<\mathrm{Ga} / \mathrm{N}<1.5$ at $T_{s}=700{ }^{\circ} \mathrm{C}$. The results of the electron mobility at $4 \mathrm{~K}$ over $\mathrm{Ga} / \mathrm{N}$ are given in Figure 4.5. A strong dependence on the Ga-flux is observed with a maximum mobility at the bilayer-droplet transition. This is comparable to reports on transport in bulk GaN $[41,22]$. The relative change of the mobility for 2DEGs is, however, much stronger than for bulk material. This suggests that Ga-flux not only has an influence on the bulk properties but also a high 


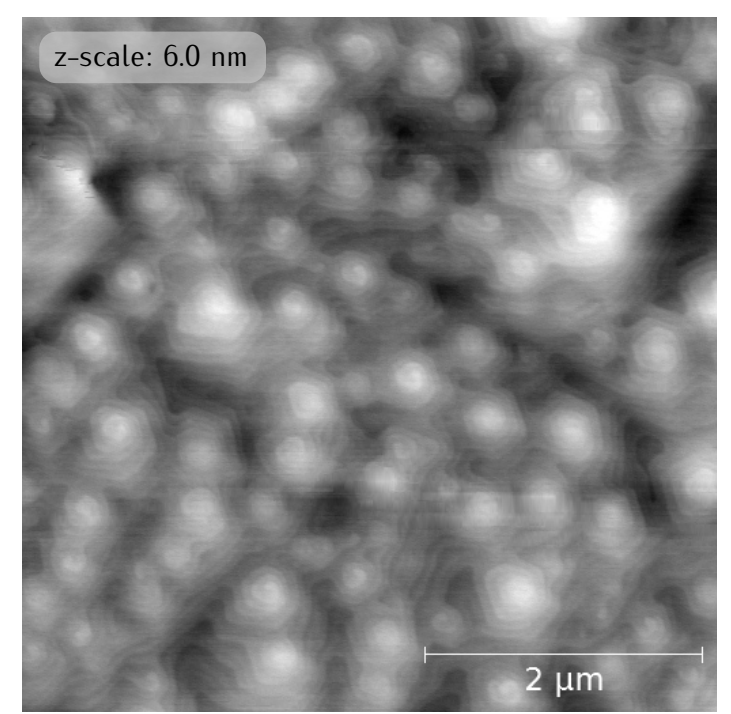

Figure 4.4

Atomic force micrograph of GaN (0001) grown under Ga bilayer stabilized conditions. Spiral hillocks with atomically flat terraces are observed.

impact on the surface morphology which determines the interface quality in heterostructures.

In order to identify the high temperature limit given by GaN decomposition, the growth rate depending on temperature was measured by RHEED oscillations under stoichiometric growth conditions. The growth rate decreases above $750{ }^{\circ} \mathrm{C}$ as shown in Figure 4.6. This is in reasonable agreement with the decomposition of $\mathrm{GaN}$ in $\mathrm{MBE}$ under an activated nitrogen environment [117]. The decomposition temperature under metal-rich conditions is, however, lower due a decreased kinetic barrier for decomposition under a metal adlayer. A reduction in the decomposition temperature from to $720^{\circ} \mathrm{C}$ has

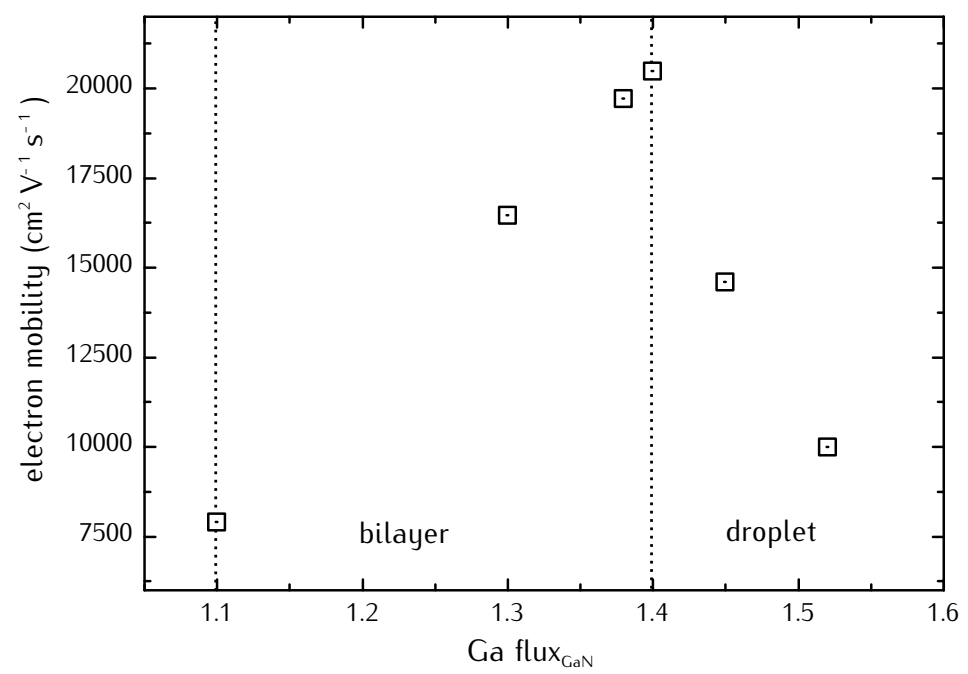

Figure 4.5: Electron mobility in a $250 \mathrm{~nm} \mathrm{GaN} / 25 \mathrm{~nm} \mathrm{Al}_{0.15} \mathrm{Ga}_{0.85} \mathrm{~N} / 50 \mathrm{~nm} \mathrm{GaN}$ structure over Ga-flux. With increasing ratio the mobility increases in the bilayer regime and decreases under droplet conditions. The highest mobility is obtained at the bilayer-droplet transition. 


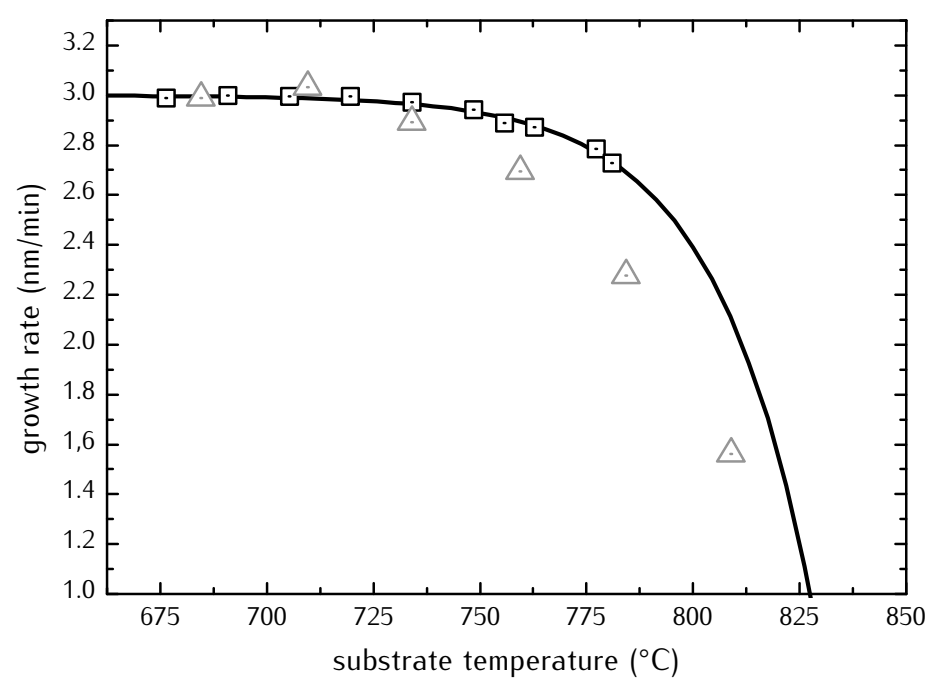

Figure 4.6: Decrease of the growth rate at higher substrate temperatures indicating decomposition. Squares: data obtained by RHEED oscillations, triangles: normalized data by VanMil et.al. [25].

been reported [43]. The substrate temperature under the applied nitrogen conditions for Ga-rich growth is thus limited to $720^{\circ} \mathrm{C}$.

It has to be noted, that the substrate temperature values given in this work are based on the absolute temperature calibration presented in Appendix C. They do not represent the temperature readings on the MBE setup used in this work. During the optimization process, the control temperature was adjusted according to the Ga coverage with respect to the applied nitrogen conditions in order to reproduce growth conditions. After the experimental phase of this work, the temperature calibration given in Appendix $C$ was applied to all results to provide absolute and comparable substrate temperature values.

The impact of the substrate temperature on the electron mobility was not studied in detail in this work. A substrate temperature of $700{ }^{\circ} \mathrm{C}$ was chosen as the surface diffusion of gallium is highest at this temperature (see Figure 1.9). All heterostructures presented in the following chapters are based on GaN buffer layers grown at $T_{s}=700{ }^{\circ} \mathrm{C}$ with $\mathrm{Ga} / \mathrm{N}=1.4$ at the bilayer-droplet transition.

\subsection{MODULATED GROWTH}

A strong dependence of the mobility on the Ga-flux with a maximum at the bilayer-droplet transition was presented in the previous section. This illustrates the importance of constant growth conditions. They are, strictly speaking, met by a single Ga-flux at a given substrate temperature. Even with extensive calibration of all parameters, this is difficult to achieve. Temperature inhomogeneities on the surface and changes in the Ga-flux over 


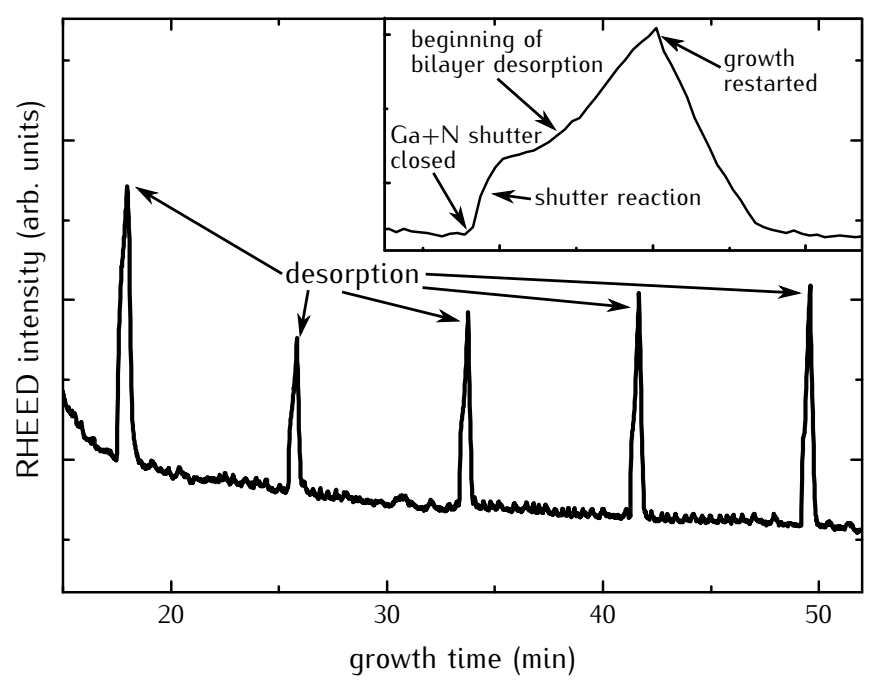

Figure 4.7: RHEED intensity during modulated growth. The growth is interrupted every $7.5 \mathrm{~min}$ for desorption of excess Ga. The inset shows one desorption step. After all shutters a close, the intensity increases abruptly which is not related to desorption. As soon as desorption is indicated, the growth is restarted.

time make constant growth conditions impossible over longer periods and over the whole sample. By applying a modulated growth technique, the impact of such deviation was reduced.

Modulated growth techniques are often employed for growth on large substrates to counteract the droplet formation in colder areas with lower desorption rates [50]. Most commonly used is the metal modulated epitaxy (MME). Here, the metallic adlayer is fully incorporated during intervals where only nitrogen is supplied. This allows to compensate differences in the metal coverage on a surface due to temperature inhomogeneities. In this work, a different approach was applied because of the small size of grown samples, where large area temperature inhomogeneities are negligible.

As it was demonstrated, the surface should be covered with a Ga bilayer during optimum growth. The complete removal of the metal is therefore not desired as the growth would restart under non ideal conditions. An interruption should allow enough time for the reduction of excess Ga without disintegrating the Ga bilayer. This can be monitored by the RHEED intensity, which increases when the bilayer desorption or incorporation starts (see Figure 2.14). During an interruption step, the RHEED intensity was thus controlled and the growth was restarted as soon as the RHEED intensity increased. The desorption rate is smaller than the growth rate at the applied substrate temperature. The interruption is this longer for desorption than for $M M E$, where growth continues during interruption, as only the metal shutter is closed. Desorption steps thus provide a longer time to react on changes in the RHEED intensity compare to MME. This allows for a better control of the growth, and frequent desorption steps were applied in this 


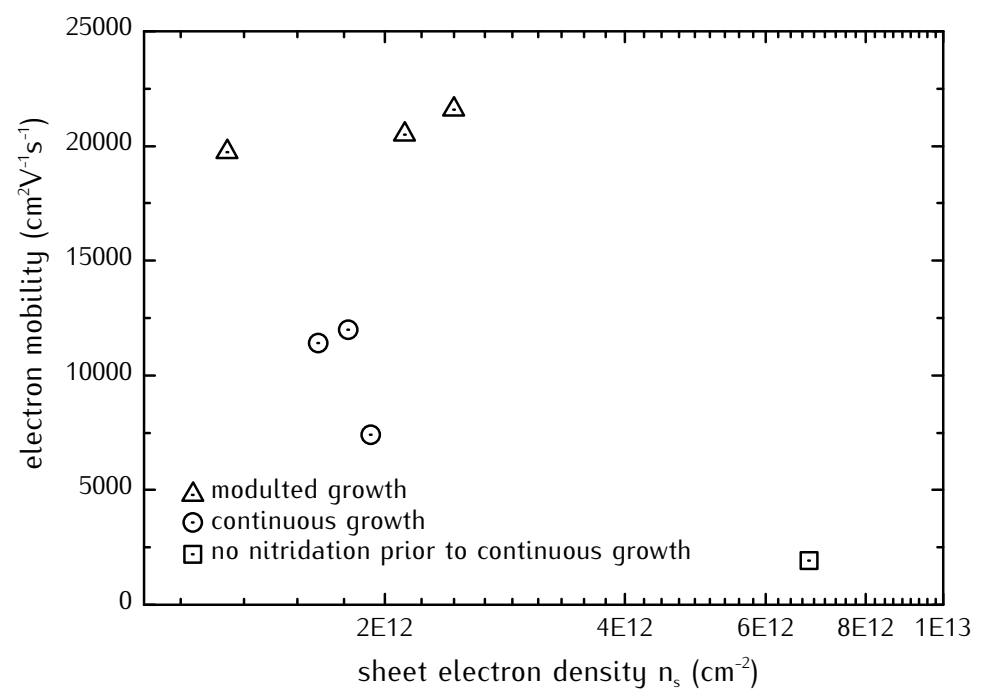

Figure 4.8: Electron mobility over carrier concentration at $4 \mathrm{~K}$ for selected GaN/ AlGaN structures indicating the impact of active nitrogen treatment before growth and modulated growth. The highest mobilities were obtained for nitrogen treatment with modulated GaN growth with a $\mathrm{Ga}$ supply at the bilayer-droplet transition.

work instead of MME.

An example of the RHEED intensity during growth of a thick GaN layer with desorption steps is given in Figure 4.7. The inset shows one exemplary desorption step. The growth is restarted as soon as the intensity indicates a desorption of the Ga bilayer. To minimize Ga accumulation, the growth should be interrupted frequently. However, the number of growth interruptions has to be limited because the cell shutters might suffer from many open/close cycles. In addition, a high amount of interruptions decreases the effective growth rate. As a balance between small Ga accumulation and a reasonable growth/desorption ratio, growth periods of $7.5 \mathrm{~min}$ were chosen. The samples produced with this modulated technique showed an increased homogeneity and no gallium droplets on large parts of grown samples even under highly Ga rich growth conditions.

Another benefit of this technique is that the Ga coverage is checked at each desorption step. Changes in the gallium flux and the substrate temperature were thus detected and compensated. In this way, the reproducibility increased. Furthermore, an increase of the electron mobility in GaN/AlGaN 2DEG structures was achieved as shown in Figure 4.8. 


\title{
ALUMINIUM GALLIUM NITRIDE HETEROSTRUCTURES
}

\begin{abstract}
The electron mobility in GaN/AlGaN 2DEG structures is mainly influenced by the quality of GaN. The impact of growth optimization on the mobility was therefore presented in the previous chapter. This chapter addresses the growth of AlGaN only briefly. It pays closer attention to the dependencies of the electronic properties on the heterostructure design. The dominating scattering process at low-temperature is identified by an analysis of the magnetotransport data. A substantial part of this study is based on results obtained by Murat Sivis during his diploma thesis. The last section presents the results of an investigation on the sensitivity of grown GaN/AlGaN structures to ionic solutions.
\end{abstract}

\subsection{GROWTH CONDITIONS AND STRUCTURAL QUALITY}

Two dimensional electron gases (2DEGs) form along the GaN/AlGaN interface. Electrons in the channel mainly move on the GaN side of the interface and have only a small penetration depth into the AlGaN layer (see Section 3.1). The focus of AlGaN growth optimization with respect to the electron mobility in 2DEGs is thus on the interface and not as much on the bulk quality of AlGaN. In the following, only the optimization of heterostructures with thin AlGaN layers and low aluminum content is considered as they result in the highest mobilities (see Section 5.2).

The growth of ternary compounds is always a compromise between the optimal growth conditions of the two binary materials involved. Compared to $\mathrm{GaN}$ growth, this implies a higher growth temperature for $\mathrm{AlGaN}$, because of the higher thermal stability of AIN. Just as for GaN growth, the optimum growth temperature at half the melting point is not available due to decomposition under MBE conditions. On this account, Ga bilayer stabilized growth conditions, which enhance the surface diffusion of nitrogen, were applied during AlGaN growth. Growth under Al-rich conditions is not possi- 
Figure 5.1

Atomic force micrograph of the GaN (0001) surface for a GaN/AlGaN/GaN heterostructure. Atomically flat terraces are observed.

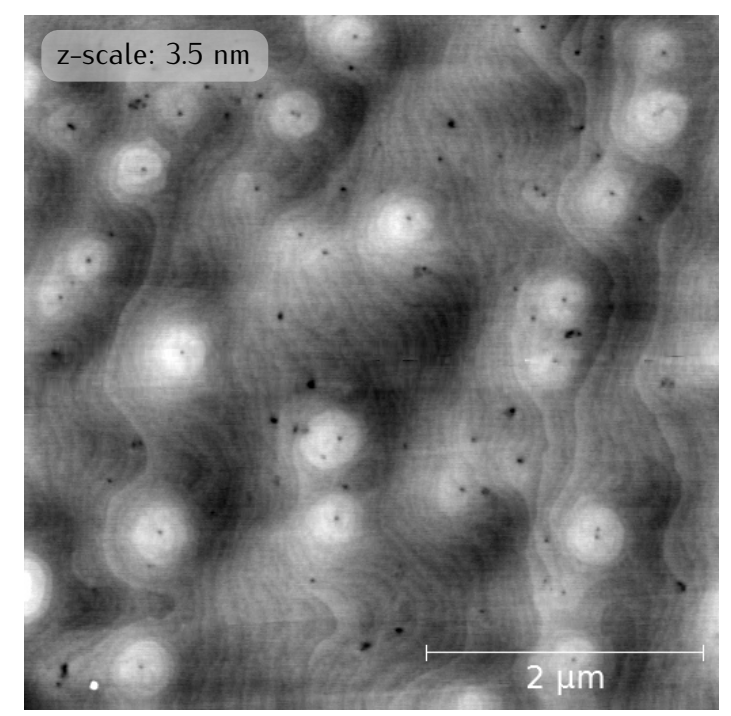

ble because aluminium preferably incorporates over gallium and only AIN grows under an aluminium adlayer. Smooth GaN/AlGaN heterostructures were grown under Ga bilayer stabilized conditions as shown in Figure 5.1. During AlGaN growth, less Ga incorporates compared to GaN growth. In principle, this has to be compensated by adjusting the applied Ga-flux. In this work, only one Ga cell was used, and a change of the Ga-flux thus requires a change of the Ga cell temperature. This results in a growth interruption which increases potential contaminations from the ambiance. The changes in the growth conditions for AlGaN growth compared to GaN growth are however small for thin layers with little Al content $(<25 \mathrm{~nm}$ and $<0.25 \%$ ). Consequently the Ga-flux and the growth temperature were kept at optimum GaN growth conditions during AlGaN growth in order to
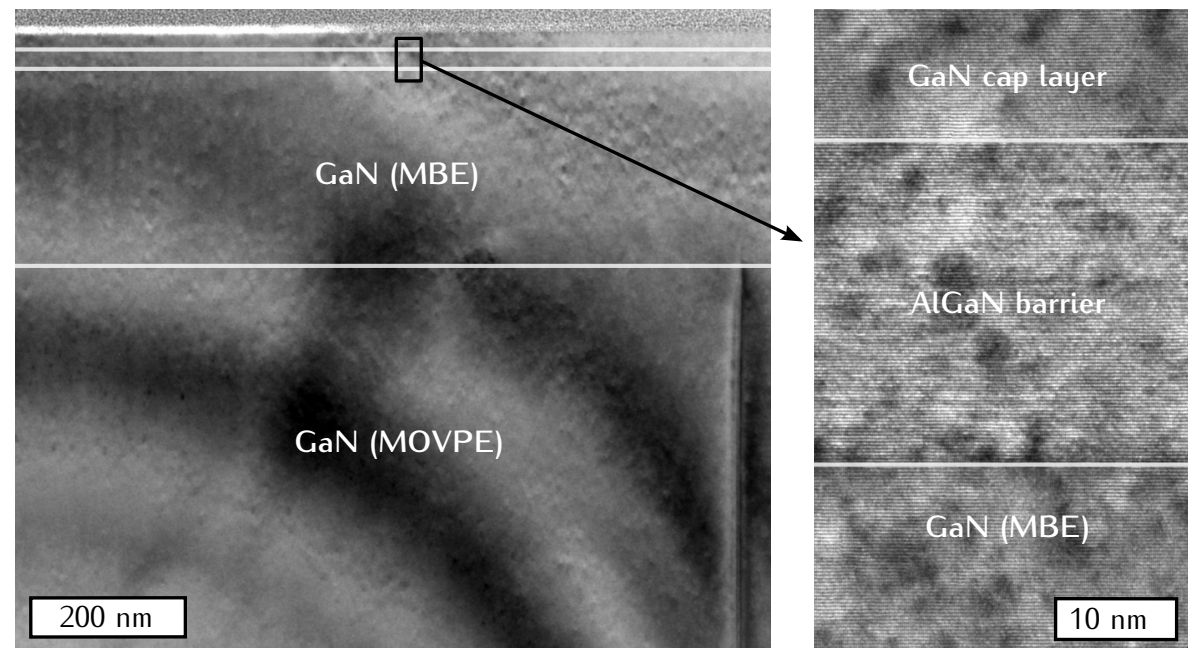

Figure 5.2: Transmission electron micrograph of GaN/AlGaN/GaN sample G0490. No additional dislocations form at the heterostructure interfaces. Sharp transitions between GaN and AlGaN are observed. 
minimize the growth interruption time. TEM images indicated sharp, smooth interfaces with no additional dislocations for the applied growth conditions as shown in Figure 5.2.

Apart from the interface quality, the Al content is of importance for GaN/Al$\mathrm{GaN}$ heterostructures. Aluminium is preferably incorporated over Ga due to the higher bond strength of $\mathrm{Al}-\mathrm{N}$ compared to Ga-N. Deosprtion of $\mathrm{Al}$ can be neglected at the applied substrate temperature. This means that every $\mathrm{Al}$ atom adsorbed on the surface binds with a $\mathrm{N}$ atom before any Ga-N bond forms. The aluminium content is therefore determined by $x_{A l}=\mathrm{Al} / \mathrm{N}$ [48] under overall metal rich conditions $(\mathrm{Ga}+\mathrm{Al}>\mathrm{N})$. The $\mathrm{Al}$ concentration was thus determined from the fraction of the applied Al-flux compared to the stoichiometric flux for AIN growth. In addition, the Al content in grown layers was confirmed by X-ray diffraction measurements regularly $[120,121]$.

\subsection{GAN/ALGAN HETEROSTRUCTURE DESIGN}

The foundation of a high electron mobility $\mu$ in 2DEG heterostructures is a good crystal and interface quality. Furthermore, $\mu$ depends on the electron sheet carrier density $n_{s}$ (see Section 3.2). As will be presented in this section, the thickness of different layers and the Al content in GaN/AlGaN structures have a significant impact on $n_{s}$. Thus, the design of the heterostructure is essential to achieve the higher possible mobility for a given structural quality.

All AlGaN based heterostructures presented in this work have the same general layer stacking as illustrated in Figure 5.3. They consist of thick GaN layers grown on MOVPE templates followed by an AlGaN layer. Structures are capped with GaN layers to obtain the same surface potential, independent of the $\mathrm{Al}$ content. For such structures, a maximum low temperature electron mobility is expected around $n_{s}=2 \times 10^{12} \mathrm{~cm}^{-2}$ [122].

The electron concentration of 2DEGs was calculated using the self consistent Schrödinger-Poisson solver nextnano ${ }^{3}$ [123]. Apart from the parameters discussed in the following paragraphs, other factors will affect $n_{s}$ that have

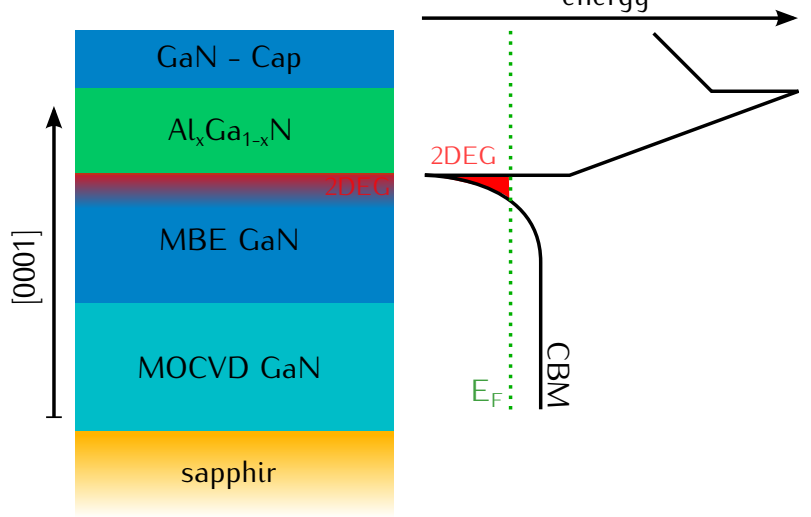

Figure 5.3

Typical GaN/AlGaN stacking used in this work together with a sketch of the conduction band minimum (CBM) indicating the 2DEG at the interface. 

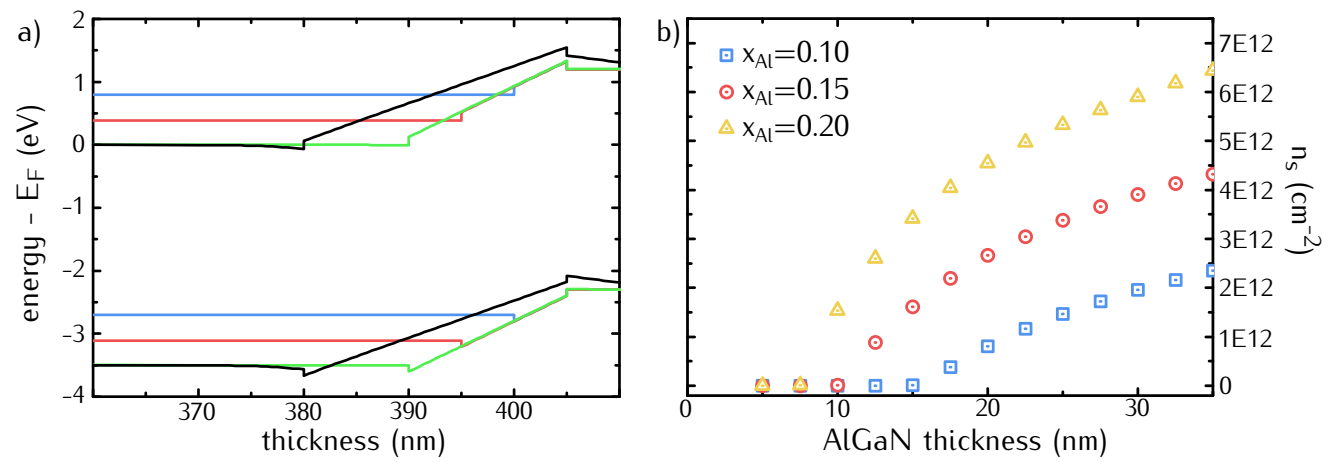

Figure 5.4: Dependence of $n_{s}$ on thickness $t_{A l G a N}$ and $x_{A l}$. a) profile of CBM and VBM for increasing AlGaN thickness. The Fermi level is pinned at the surface. Above a certain thickness CBM drops below $E_{F}$ and a 2DEG forms. For better illustration, no doping is considered in this picture. b) $n_{s}$ for different $\mathrm{Al}$ contents with varying AlGaN thickness, with doping taken into account. $n_{S}$ increases with $x_{A l}$ and $t_{A l G a N}$.

not been studied experimentally in this work. One is the position of the Fermi level at the surface. It is fixed at $1.2 \mathrm{eV}$ below the conduction band minimum [82]. Other parameters were used as provided by nextnano ${ }^{3}$.

Figure 5.4 a) shows the valence band maximum (VBM) and the conduction band minimum (CBM) profile for a GaN/AIGaN/GaN structure. Here, no doping is considered for better illustration. At the surface, $F L$ is pinned to $1.2 \mathrm{eV}$ below CBM. The bands tilt downwards with respect to the surface in the AlGaN layer according the direction of the polarization fields. As for a capacitor, the voltage difference at the boundaries of the AlGaN layer increases with increasing AlGaN thickness $t_{A l G a N}$ under the constant polarization fields. Above a certain thickness, CBM drops below FL. Electrons then occupy the free states causing a band bending in the GaN. The result is a 2DEG in an almost triangular well.

The depth of the well increases with $t_{A l G a N}$ and so does $n_{s}$ as shown in Figure 5.4 b). Here, and in the following, n-type doping is taken into account with a concentration of $n=2 \times 10^{17} \mathrm{~cm}^{-3}$. The doping concentration was determined from transport measurements and is caused by the unintentional background doping through point defects in GaN. For higher polarization fields, CBM drops below FL at thinner layers. Therefore, a 2DEG forms at thinner AlGaN layers for higher aluminium contents and $n_{s}$ increases with $x_{A l}$.

The charge of the electron channel causes an additional field between the 2DEG and the surface. The strength of the field depends on $n_{s}$ and is of the opposite direction of the polarization field. It thus decreases the effective field in the AlGaN layer. In addition it causes an upward tilt of CBM within the GaN cap layer (see black line in Figure 5.4 a)). Both, the reduction of the effective field in the AlGaN layer and the tilt of the band within the cap layer, affect the sheet carrier density $n_{s}$, which then again results in 

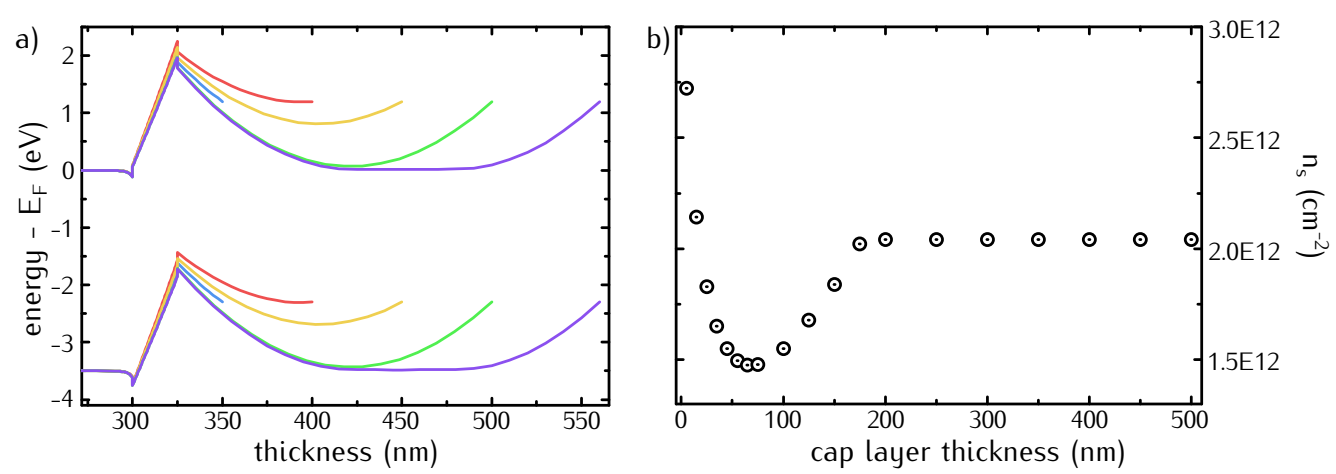

Figure 5.5: Dependence of $n_{s}$ on GaN cap layer thickness, with doping. a) CMB and $\mathrm{VMB}$ profile for different cap thicknesses. The screening of the polarization and the surface charge results in a band bending within the cap layer. For thick cap layers, both charges are fully screened. b) $n_{s}$ depending on cap layer thickness. $n_{s}$ saturates for thick cap layers.

a change of the field itself. Self consistent calculations of this mechanism show an effective reduction of $n_{s}$ with increasing GaN cap layer thickness up to a certain GaN thickness as shown in Figure 5.5 b). If the thickness is increased further, the bands within the GaN bow due to screening of the surface potential by electrons from cap layer as shown in Figure 5.5 a). Ultimately, $n_{s}$ becomes independent of the cap layer thickness.

It should be noted that thick cap layers are not practical. To minimize bulk contributions, the 2DEG is contacted directly (see Section 3.3). Ohmic con-

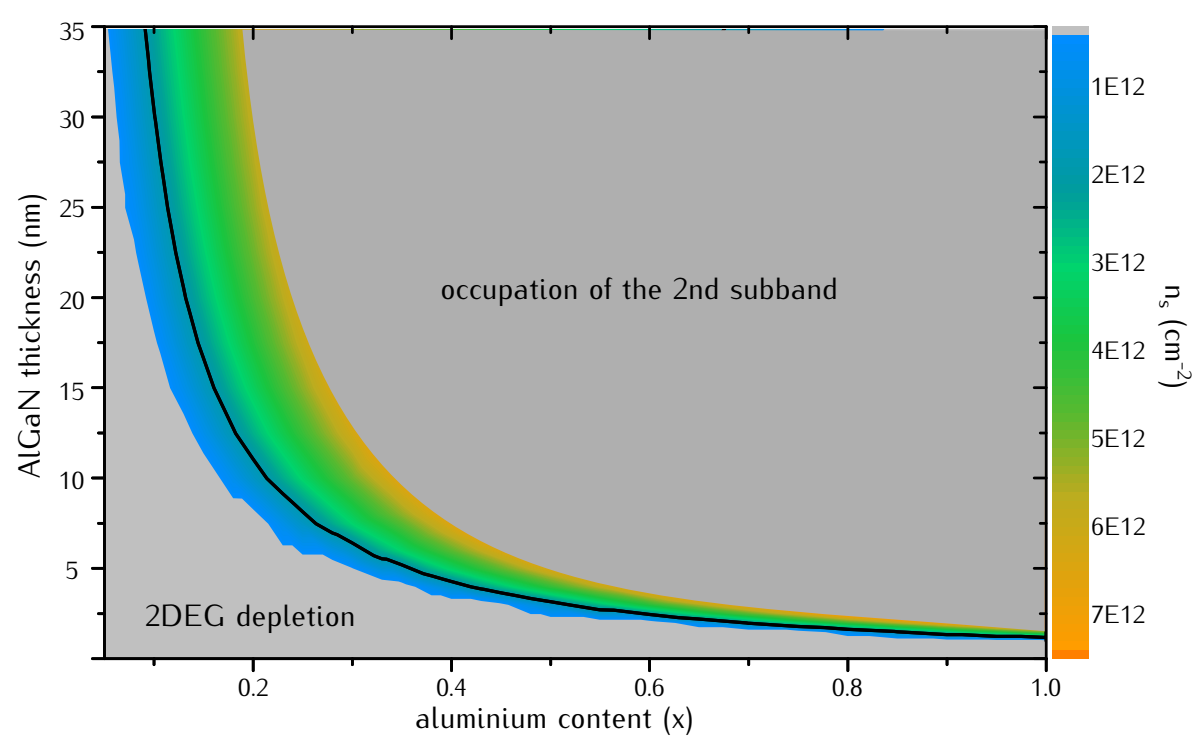

Figure 5.6: Electron concentration $n_{s}$ over $x_{A l}$ and thickness $t_{A l G a N}$ for $2 \mathrm{~nm}$ GaN cap layer. $n_{s}$ for which $\mu$ is maximal is indicated by the black line. The area of 2DEG depletion as well as the occupation of the 2nd subband are marked. 2DEGs are achieved over a wider range of $t_{A l G a N}$ for small $x_{A l}$. 
tacts were prepared by annealing a stack of metallic layers, which diffused about $75 \mathrm{~nm}$ into the material. If the 2DEG lies deeper, a direct contact requires additional processing. Up to $75 \mathrm{~nm} n_{s}$ decreases with increasing cap layer thickness, and thicker GaN cap layers were only applied to reduce $n_{s}$.

A contour plot of $n_{s}$ depending on $x_{A l}$ and $t_{A l G a N}$ is given in Figure 5.6. The 2DEG depletes for thin AlGaN layers with low Al content. For large $x_{A l}$ and $t_{A l G a N}$ the quantum well is deep enough for the second subband to drop below the Fermi energy. This should be avoided because of intersubband scattering $[124,125]$. Furthermore, it complicates the analysis of magnetotransport data, as electrons in the second subband represent an additional conduction channel with different $n_{s}$ and $\mu[126,127]$.

The sheet carrier density around which high $\mu$ is achieved is indicated in Figure 5.6 by the black line. High mobility structures are achieved for a wider range of AlGaN thicknesses at lower $x_{A l}$. It is also evident that for low $x_{A l}$ small deviations from intended heterostructure parameters have a smaller impact on $n_{S}$ than for high Al contents. A better reproducibility of the carrier densities is thus achieved at low $x_{A l}$ and the impact of fluctuations in $t_{A l G a N}$ and $x_{A l}$ on $n_{S}$ reduces. In addition, low Al contents are desired in order to reduce the effect of alloy scattering [128]. This work is therefore focused on thin AlGaN layers with low Al contents $(<25 \mathrm{~nm}$ and $<0.25 \%)$.

\section{$5 \cdot 3$ MAGNETORESISTANCE}

The two dimensional electron gas is mainly confined in the GaN buffer layer in GaN/AlGaN heterostructures. The growth optimization of GaN thus

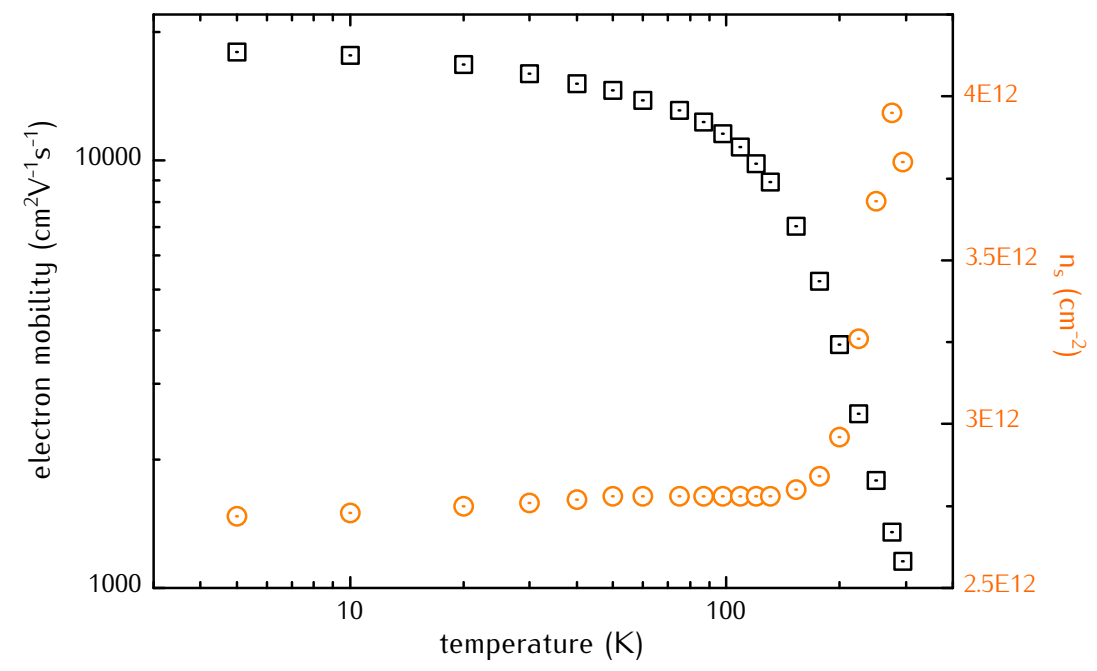

Figure 5.7: Temperature dependence of $\mu$ and $n_{s}$ of sample G0502 $\left(200 \mathrm{nmGaN} / 25 \mathrm{~nm} \mathrm{Al} 0.15 \mathrm{Ga}_{0.85} \mathrm{~N} / 5 \mathrm{nmGaN}\right)$ showing a typical 2DEG dependence with almost constant $n_{s}$ and $\mu$ at low temperatures. 


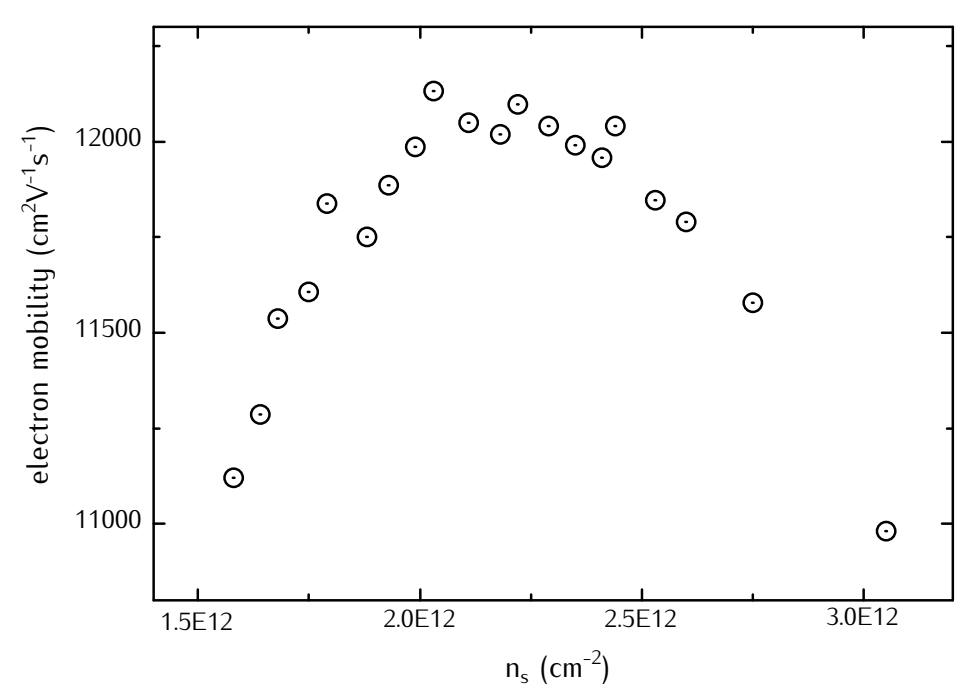

Figure 5.8: Results from gate dependent measurements of $\mu\left(n_{s}\right)$ for sample G0490 $\left(200 \mathrm{~nm} \mathrm{GaN} / 25 \mathrm{~nm} \mathrm{Al} 0.15 \mathrm{Ga}_{0.85} \mathrm{~N} / 25 \mathrm{~nm} \mathrm{GaN}\right)$. A maximum is observed at $n_{s}=2.2 \times 10^{12} \mathrm{~cm}^{-2}$ due to the impact of different scattering processes.

has the biggest impact on the 2DEG mobility. These results have therefore already been presented in Chapter 4. In the following, the magnetoresistance of selected optimized structures is discussed in order to identify the scattering mechanism that has the biggest impact on low temperature transport and limits the mobility.

The temperature dependence of $\mu$ and $n_{s}$ for a high mobility structure is given in Figure 5.7. Both $\mu$ and $n_{s}$ show a typical 2DEG behavior. At low temperatures, the sheet carrier density is almost independent of $T$. Above $120 \mathrm{~K}$ the contribution from bulk electrons sets in and $n_{s}$ increases. The electron mobility is decreased at higher temperatures due to an increased scattering rate by phonons.

The electron mobility does not only depends on the sample quality but also on the sheet carrier density (see Section 3.2). To investigate if the maximum electron mobility for the given structural quality was achieved, the dependence of $\mu$ on $n_{s}$ was studied. This was done for some structures with a top gate. If the voltage applied to the top gate is varied, the electron concentration changes and $\mu\left(n_{s}\right)$ can be measured. One result is given in Figure 5.8. For low carrier concentrations, the mobility increases with $n_{s}$ due to the improved screening of charged scatterers. For high $n_{s}$, the probability density broadens and a larger fraction of carriers is found close to the interface or even within the AlGaN. This increases the impact of scattering by interface roughness and alloy disorder, and the mobility decreases with increasing $n_{s}$. The maximum mobility is found around $n_{s}=2 \times 10^{12} \mathrm{~cm}^{-2}$, in good agreement with literature the $[88,122]$. The optimization of the electron concentration in terms of heterostructure design was thus successful. 


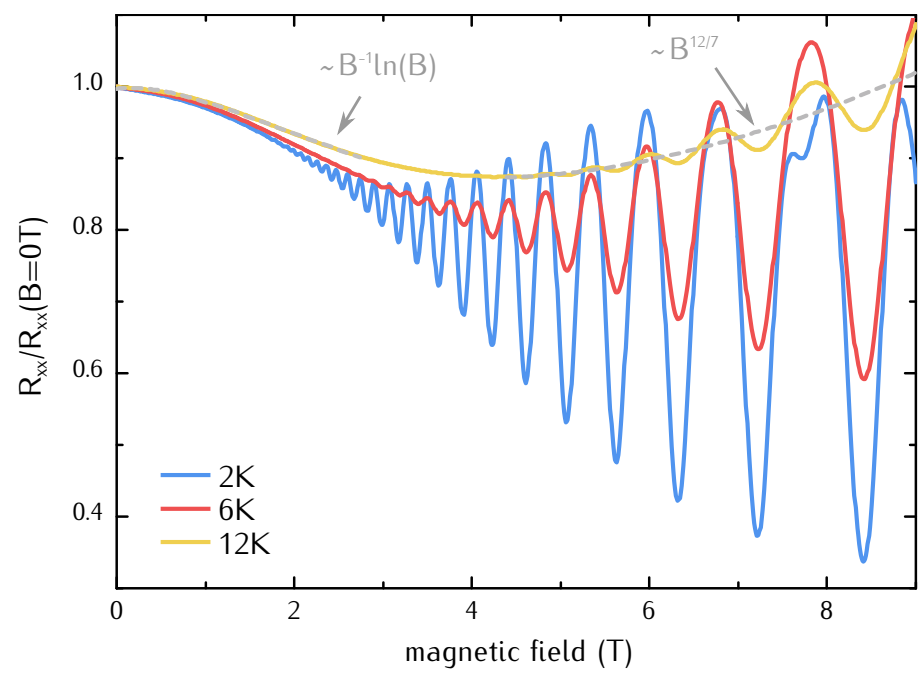

Figure 5.9: Normalized magnetoresistance of sample G502 with $\mu=21500 \mathrm{~cm}^{2} \mathrm{~V}^{-1} \mathrm{~s}^{-1}$ at $n_{s}=2.44 \times 10^{12} \mathrm{~cm}^{-2}$ at $2 \mathrm{~K}$. SdH oscillations are observed above $2 \mathrm{~T}$ at $2 \mathrm{~K}$. At $12 \mathrm{~K}$ the $B^{-1} \ln B$ dependence at low $B$-fields and the $B^{12 / 7}$ dependence for higher $B$-fields indicate the presence of a smooth scattering potential and a potential of impenetrable discs.

The highest mobility obtained during this work was $\mu=21500 \mathrm{~cm}^{2} \mathrm{~V}^{-1} \mathrm{~s}^{-1}$ with $n_{s}=2.44 \times 10^{12} \mathrm{~cm}^{-2}$ at $2 \mathrm{~K}$ for a $250 \mathrm{~nm} \mathrm{GaN} / 25 \mathrm{~nm} \mathrm{Al} 0.15 \mathrm{Ga}_{0.85} \mathrm{~N} /$ $50 \mathrm{~nm} \mathrm{GaN} \mathrm{structure.} \mathrm{The} \mathrm{magnetoresistance} \mathrm{of} \mathrm{this} \mathrm{structure} \mathrm{at} \mathrm{low} \mathrm{temper-}$ atures is shown in Figure 5.9. Clear Shubnikov-de Haas ( $\mathrm{SdH}$ ) oscillations were observed above $2 \mathrm{~T}$ and were used to obtain different parameters. The mobility was calculated from the resistivity at $B=0 \mathrm{~T}$ together with the sheet carrier density from the periodicity over $1 / B$ [100]. An effective mass of $m^{*}=0.204 m_{0}$ resulted from the temperature dependence of the amplitude [105] which is in close agreement with the literature [90]. With the knowledge of $\mu, n_{s}$ and $m^{*}$ the transport lifetime $\tau_{t r}=2.55 \mathrm{ps}$ as well as the mean free path $l_{t r}=565 \mathrm{~nm}$ were calculated from $\mu=e \tau_{t r} / m^{*}$ and $l_{e}=v_{F} \tau_{t r}$. In addition, a quantum life time $\tau_{q}=0.83$ ps was gained from the amplitude of $\mathrm{SDH}$ oscillations over magnetic field [105].

The dominating scattering mechanism can be identified from $\tau_{t r} / \tau_{q}$. This ratio is a measure of the average scattering angle. The transport relaxation time is derived from the mobility, which is determined by the motion of carriers along an applied field. Therefore, large angle scattering processes cause a stronger reduction of $\tau_{t r}$ than small angle scattering, while the impact on $\tau_{q}$ is equal for every scattering event. For the presented structure, the ratio is $\tau_{t r} / \tau_{q}=6.7$, which results from small angle scattering and suggests scattering dominated by dislocations [106]. Furthermore, the mean free path corresponds to a density of $3.13 \times 10^{8} \mathrm{~cm}^{-2}$, which is close to the dislocation density of the substrate. Therefore, the scattering at dislocations originating from the substrate is the main elastic scattering mechanism at 
low temperatures.

This indicates that the growth optimization was successful. No further dislocation were incorporated during MBE growth and the quality of the substrate mainly limits the mobility.

As the amplitude of the $\mathrm{SdH}$ oscillations weakens with increases temperature, another contribution to the magnetoresistance is observed as shown for $12 \mathrm{~K}$ in Figure 5.9. For the presented measurement two different dependencies are found. At low magnetic fields the magnetoresistance is proportional to $B^{-1} \ln (B)$ and at high fields to $B^{12 / 7}$. This $R_{x x}(B)$ dependence is observed for 2D carrier transport affected by two potentials [114]. One potential is described by a random distribution of impenetrable discs with radius $a$ and mean distance $l_{a}$. The other describes smooth potential changes over a radius $d$ and the mean distance $l_{d}$. The model is applicable if $a \ll d$ and $l_{a} \ll l_{d}$.

In order identify the origins of the two potentials, the impact of a threading dislocation and atomic steps from surface roughness on the conduction band minimum (CBM) was calculated by two dimensional Schrödinger Poisson calculations. This allows to analyze the potential change along the GaN/Al$\mathrm{GaN}$ interface, i.e. the moving direction of the accumulated electrons.

The atomic step results in a barrier in the minimum of CBM along the interface as shown in Figure 5.10. The height of the barrier is $0.01 \mathrm{eV}$, and it extents about $3 \mathrm{~nm}$ around the step. A dislocation in GaN can be approximated by a line of ionized point defects with acceptor character at $2.2 \mathrm{eV}$ below the conduction band minimum [89]. Such a point defect can

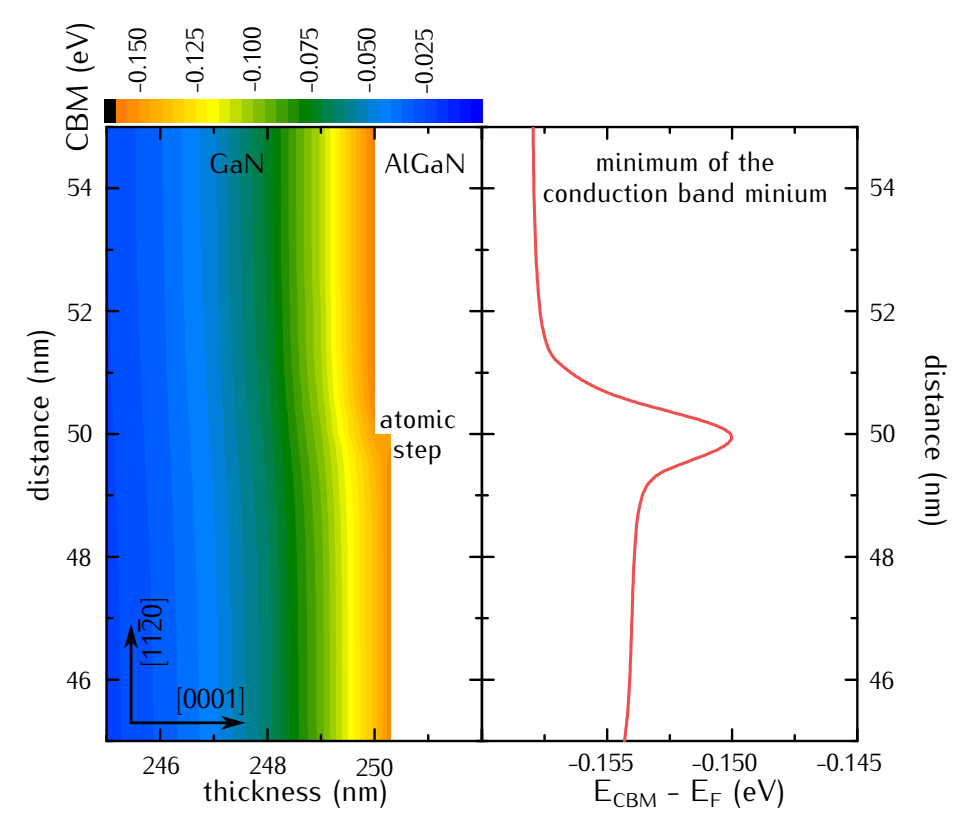

Figure 5.10: Impact of an atomic step on the 2D CBM profile. The minimum of CBM represents the energy of the deepest point of the quantum well. The step induces a potential barrier with a lateral extension of about $3 \mathrm{~nm}$. 


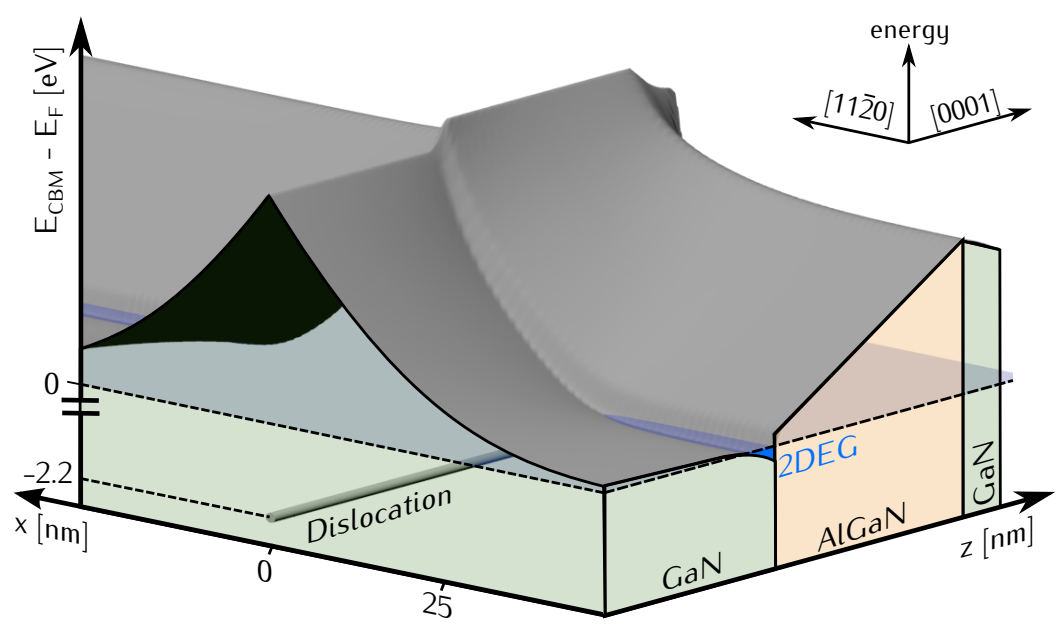

Figure 5.11: Impact of a dislocation on the 2D CBM profile. The dislocation was approximated by a line of acceptor like point defects [89]. The 2DEG is depleted around the dislocation with a radius of $25 \mathrm{~nm}$.

be found every two monolayers along the c-direction [89]. In Figure 5.11, the profile of CBM along the interface for a dislocation penetrating the 2DEG is given in a 3D plot. The dislocation pulls the Fermi level above CBM and thus depletes the 2DEG for about $50 \mathrm{~nm}$. The increase around the dislocation can be considered sharp compared to the depletion length because it is screened by the high electron concentration in the 2DEG.

Figure 5.12 shows the impact of a dislocation and atomic steps on minimum of CBM. The plotted distance was chosen according the mean free path of $565 \mathrm{~nm}$ that was obtained from magnetotransport measurements presented

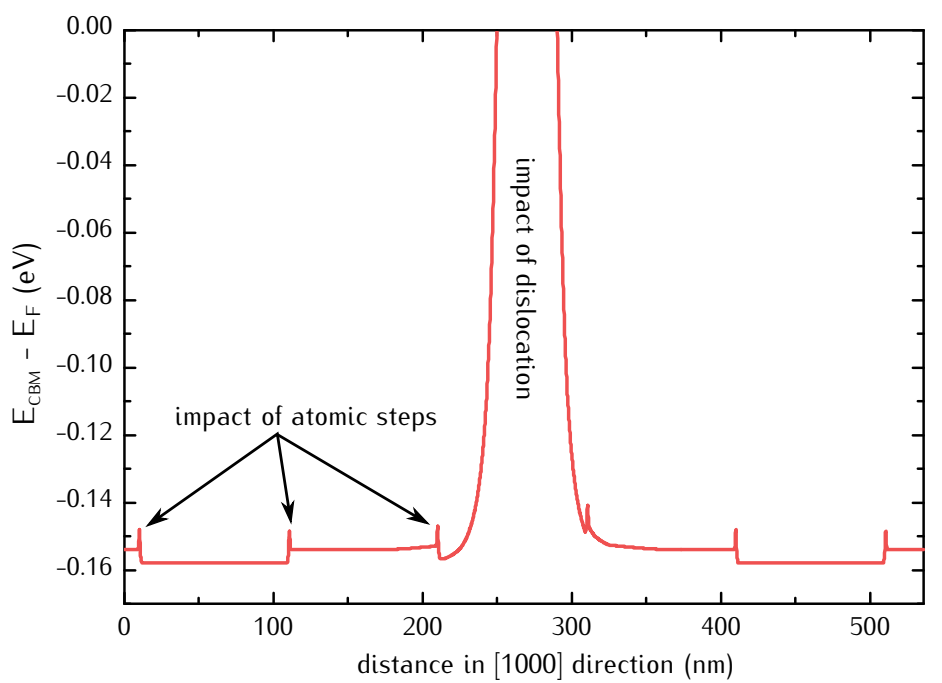

Figure 5.12: Impact of a dislocation and atomic steps on the CBM. The spacing between steps was taken from atomic force micrographs. This graph is not the result of a single calculation and is based on the results of a single step and one dislocation. 
previously. Because dislocations were identified as the dominant scattering mechanism, this distance equals the spacing between two dislocations. Therefore, only one dislocation is plotted. The mean terrace width between atomic step is in the order of $100 \mathrm{~nm}$ as was measured by AFM.

It is evident, that dislocations can be considered as impenetrable disc potentials because they fully deplete the 2DEG. Therefore, dislocations represent the potential of scatterers with radius $a$ and mean distance $l_{a}$. Atomic steps induce only small changes in CBM and are closer together than the dislocations. They are thus not the source of the smooth potential which would imply $d \gg a$ and $l_{d} \gg l_{a}$. The smooth potential thus has to arise from other sources with diameters of at least $50 \mathrm{~nm}$ and a mean separation well above $565 \mathrm{~nm}$. Possible sources might be inhomogeneities in the AlGaN thickness or small fluctuations of the Al concentration.

\subsection{APPLICATION: IONIC AND BIOCHEMICAL SENSING}

During this work, a collaboration with the University of Western Australia (UWA) in Perth was started. The aim is to optimize GaN/AlGaN heterostructures for ion and biomedical sensing applications. In this chapter, a brief introduction to the topic is given. To achieve high sensitivity, GaN/AlGaN structures were modeled and then grown according to the results. The experimental results are compared to MOVPE grown samples from previous works [129, 130].

Sensors are a promising application for nitride based 2DEG structures. Compared to GaAs based sensors, nitrides can be used in a broader range of applications because of their chemical stability and low toxicity. Sensitivity to gases, pressure, ions and more has been successfully demonstrated for GaN/AlGaN structures [131]. Applications in biology and medicine are realized by a functionalization of the surface with cells and molecules as depicted in Figure 5.13. Here, the chemical response of a cell attached

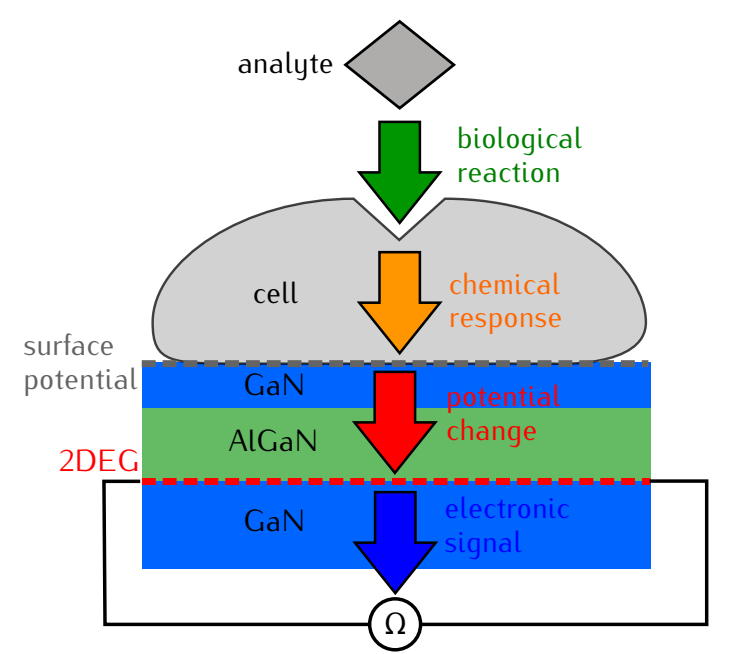

Figure 5.13

Schematic a GaN/AlGaN sensor with a functionalized surface: The analyte causes a biological reaction in a cell which results in a chemical response. This response modifies the surface potential which causes a change in the carrier density of the 2DEG and thus a change in the resistance. 


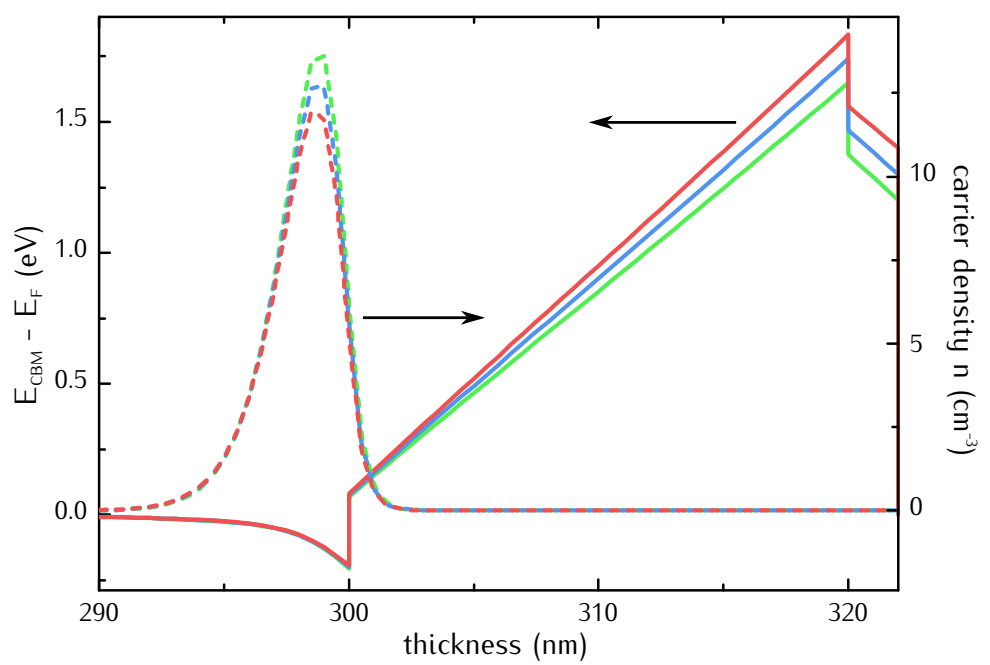

Figure 5.14: Conduction band minimum and $n$ for different surface potentials. A rise in the surface charge results in a decreased carrier density.

to the semiconductor surface to a biological reaction is detected. Sensing effects of functionalized nitride 2DEGs for glucose, kidney marker injury molecules, prostate cancer and DNA have been reported [132].

The basic sensing effect of AlGaN/GaN heterostructures is easily comprehensible. The carrier concentration of the 2DEG channel is related to the surface potential. When ions are deposited on a GaN or AlGaN surface, the surface charge is modified. This leads to a change in the band profile and thus to a change of the carrier concentration in the 2DEG as shown in Figure 5.14. As a result, a change in the conductivity is measured, which will depend on the type and amount of ions on top of the structure. This allows for truly gateless sensing devices.

The general quality of a device in terms of sensitivity can be probed by

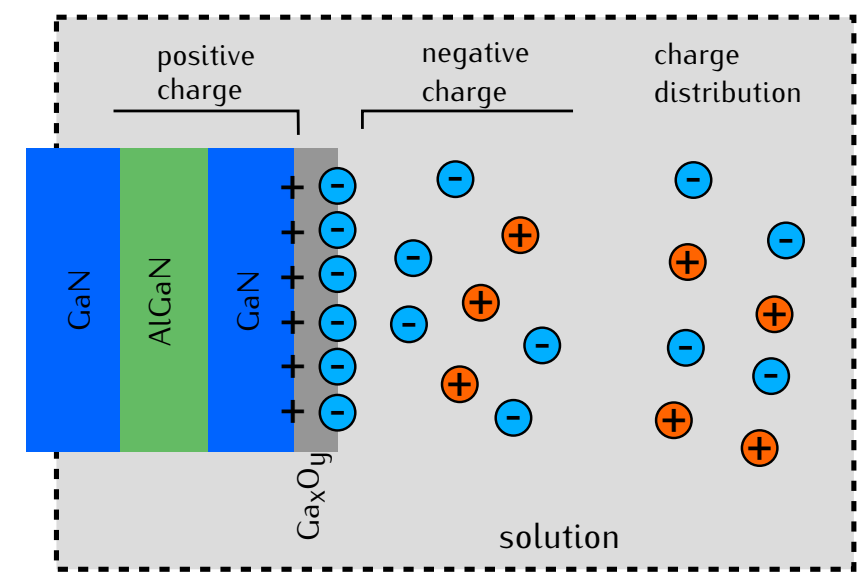

Figure 5.15: Charge distribution of a ionic solution over an GaN/AlGaN heterostructure [133]. Negative ions are accumulated at the surface due to the positive charge of the oxide. Therefore only negative ions are detected. 
the conductivity change in ionic solutions. For other applications using functionalized surfaces, the ionic reaction within a cell is probed and the underlying mechanism is the same. Thus, devices that are highly sensitive to ionic solutions will also be good sensors in biomedical applications, given that they are biocompatible.

Only a sensitivity to negative ions was reported for the used setup [129]. This results from the donor like surface states produced by gallium oxide on GaN surfaces. Their positive charge results in a redistribution of ions in solutions, where negative ions accumulate at the surface as shown in Figure 5.15. The results for $\mathrm{pH}$ sensing thus give the concentration of negative ions in a solution.

\subsubsection{Heterostructure modeling}

Prior to growth, the optimum heterostructure design for high sensitivity was calculated. In the chosen measurement configuration, exposure of Ga-face GaN/AlGaN layers to ionic solutions leads to a positive shift of the surface potential and thus to a reduction of $n_{s}$. This change $\Delta n_{s}$ was calculated using nextnano ${ }^{3}$ by specifying appropriate boundary conditions with an effective voltage change of $+100 \mathrm{meV}$ at the surface. For a realistic figure of merit, the change in $n_{s}$ was then normalized to the overall carrier concentration including bulk carriers. Results of the sensitivity as a function of AlGaN thickness and composition are given as $\log \left(100 \Delta n_{s} / n\right)$ in Figure 5.16.

The results show regions of markedly different sensitivity, with a strong dependence on AlGaN thickness and composition. The sensitivity increases when the distance between surface and 2DEG is reduced. The depletion of

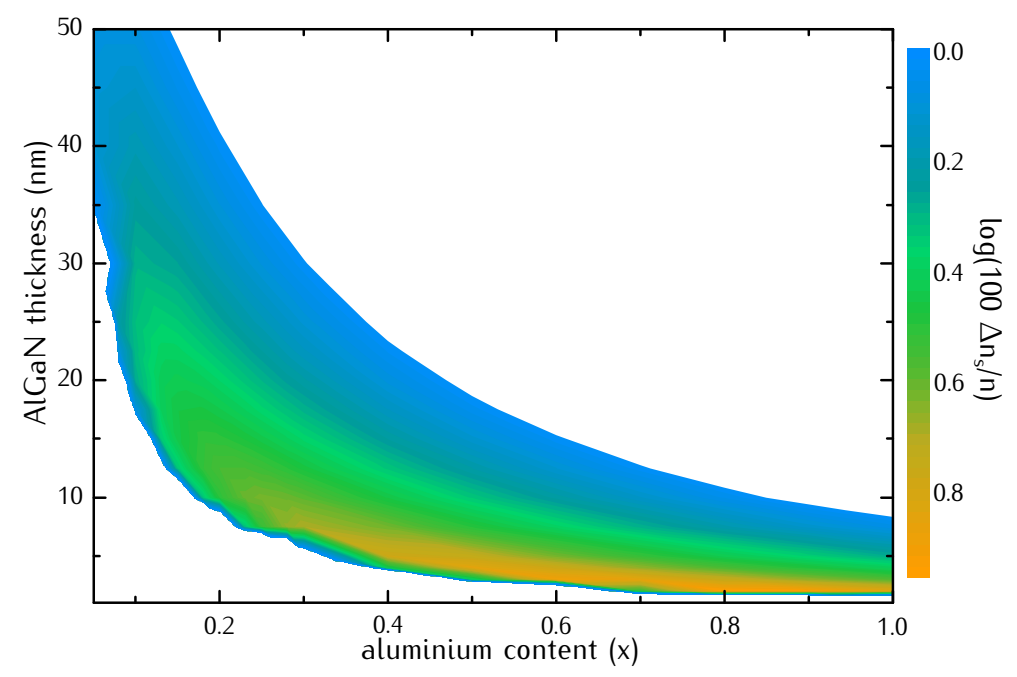

Figure 5.16: Calculated change of $n_{s}$ over aluminium content and AlGaN thickness in a GaN/AlGaN/GaN structure with $2 \mathrm{~nm}$ cap for a change of the surface potential of $+100 \mathrm{meV}$. 
the 2DEG results in a sharp threshold edge at thin AlGaN layers depending on the Al content. For thick layers, the surface charge still modulates the amount of carriers in the channel, but the relative change reflected in the sensitivity is small because of higher carrier concentrations. The most promising structures operate close to the threshold for AlGaN layers below $10 \mathrm{~nm}$ in thickness. An increased distance between 2DEG and the surface reduces the response. The GaN cap layers were therefore kept thin with a thickness of $2 \mathrm{~nm}$.

The presented modeling results only account for the carrier concentration while in experiments the conductivity is measured. The dependence of electron mobility $\mu$ on the carrier concentration $n_{s}$ could therefore lead to different experimental results. Details of $\mu\left(n_{s}\right)$ depend on the interplay of different scattering contributions and vary with device structure and material quality (see Section 3.2). A more sophisticated treatment of the device sensitivity would thus require a precise knowledge of $\mu\left(n_{s}\right)$ for each particular sample. A more basic estimation is presented in the following.

The electron mobility has a maximum depending on $n_{s}$ due to different scattering mechanisms. As the devices are operated at room temperature, scattering by phonons dominates the transport. Here, $\mu\left(n_{s}\right) \propto n_{s}^{0.5}$ is expected for low $n_{s}$ and $\mu\left(n_{s}\right) \propto n_{s}^{-0.5}$ for high $n_{s}$ [134]. Based on this consideration three different $\mu\left(n_{s}\right)$ relations have been modeled and applied to the calculated values of $n_{s}$ and $n$. Model 1 shows a high maximum room temperature mobility of $\mu=1800 \mathrm{~cm}^{2} V^{-1} \mathrm{~s}^{-1}$ at $n_{s}=4 \times 10^{12} \mathrm{~cm}^{-2}$, model 2 and $3 \mathrm{im}-$ ply a lower mobility of $\mu=1000 \mathrm{~cm}^{2} \mathrm{~V}^{-1} \mathrm{~s}^{-1}$ at $n_{\mathrm{s}}=1 \times 10^{12} \mathrm{~cm}^{-2}$ and at $n_{s}=1 \times 10^{13} \mathrm{~cm}^{-2}$. The three models therefore cover a wide range of possi-

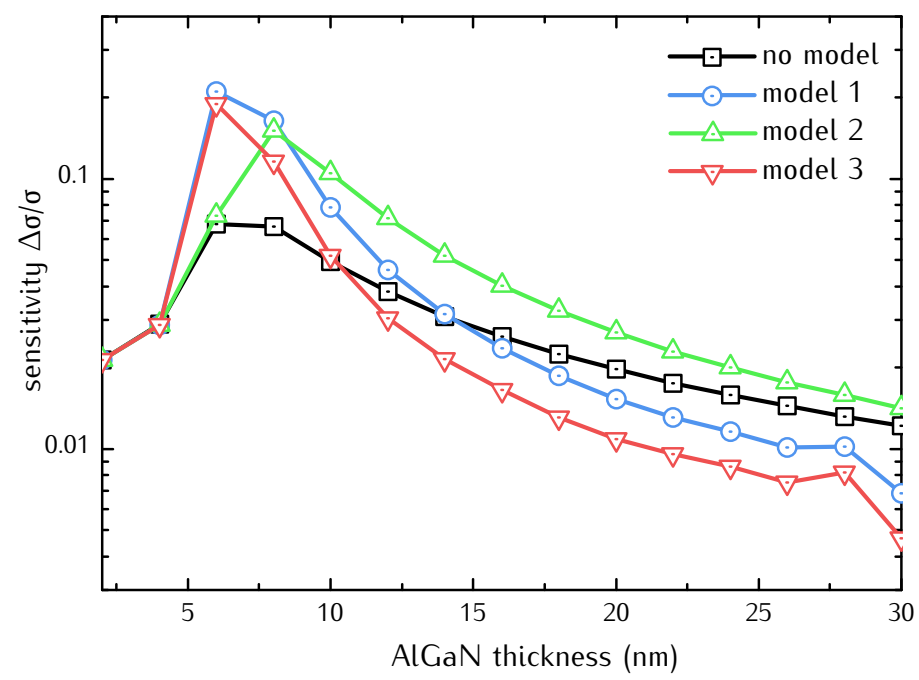

Figure 5.17: Normalized change of the conductivity of a $\mathrm{Al}_{0.2} \mathrm{Ga}_{0.8} \mathrm{~N}$ structure with $2 \mathrm{~nm}$ cap over varying AlGaN thickness. Three different estimated $\mu\left(n_{s}\right)$ dependencies are applied. The sensitivity for thin AlGaN layers is underestimated if only $\Delta n_{s} / n$ is considered. 


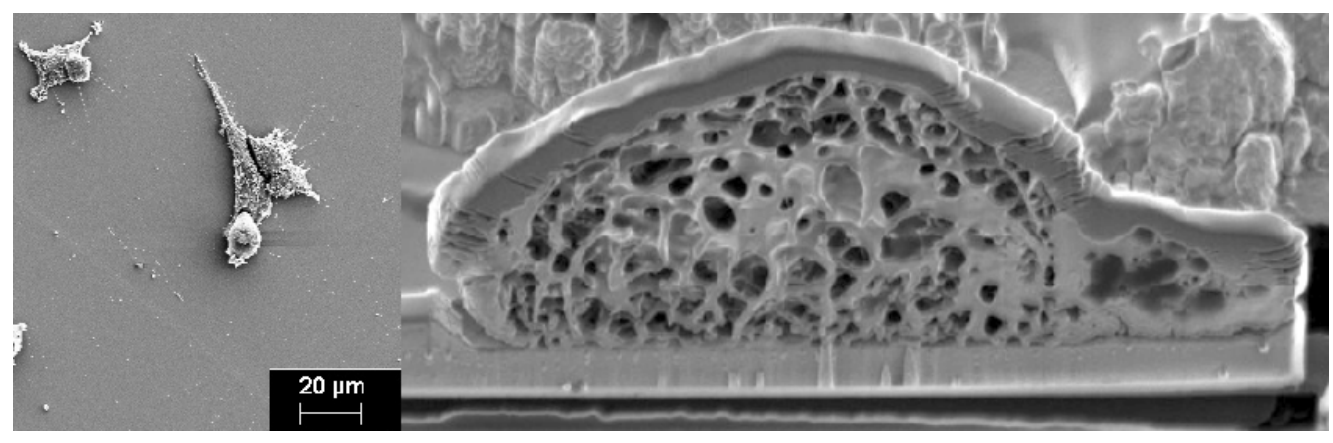

Figure 5.18: Scanning electron micrograph of cells on a AlGaN (0001) surface.

ble $\mu\left(n_{s}\right)$ dependencies.

The results of $\Delta \sigma / \sigma$ over AlGaN thickness for $x_{A l}=0.2$ for the three models are shown in Figure 5.17 and compared to the result taking only a change in the sheet carrier density into account. The general trend in the sensitivity does not change when the mobility is taken into account. However, the sensitivity is underestimated for thin AlGaN layers for all mobility models, and overestimated at thick AlGaN layers depending on the model. This emphasizes the importance of thin AlGaN layers for highly sensitive GaN/AlGaN structures.

\subsubsection{Experimental results}

First the biocompatibily of grown structures was investigated [133]. The growth of cells on a GaN surface and on AlGaN surfaces with different Al contents was compared to the cell growth in a control well over a period of 14 days. In addition, the percentage of dead cells was checked during the first 5 days. The difference of cell growth on grown structures was small compared to the control well. However, the ratio of dead cells increased with the Al content. GaN capped samples are thus more promising for medical applications than sensors with AlGaN surfaces. Furthermore, electron micrographs attested a strong attachment of the cells to all structures as the example in Figure 5.18 shows.

The sensitivity measurements were performed using a four point bar configuration (Figure 5.19), with sensing areas of $1.5 \mathrm{~mm}$ in diameter, at constant

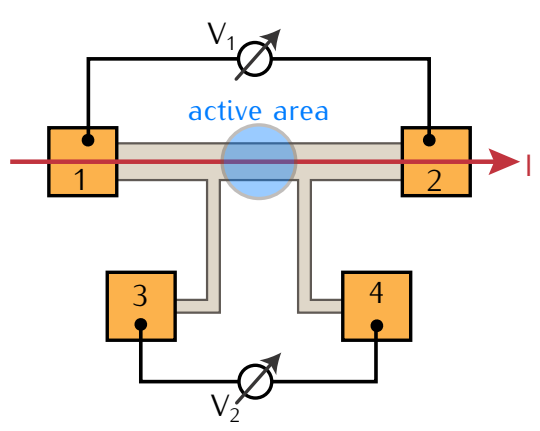

Figure 5.19

Four point probe setup for sensitivity measurements. 


\begin{tabular}{|c|c|c|c|c|c|}
\hline $\begin{array}{l}\text { Sample } \\
\text { ID }\end{array}$ & $\begin{array}{l}\text { Growth } \\
\text { method }\end{array}$ & Structure & Sens. & $\mu\left(\mathrm{cm}^{2} / \mathrm{Vs}\right)$ & Geometry \\
\hline A & MBE & $\begin{array}{l}10 \mathrm{~nm} \quad \mathrm{Al}_{0.3} \mathrm{Ga}_{0.7} \mathrm{~N} / \\
2 \mathrm{~nm} \mathrm{GaN}\end{array}$ & $29 \%$ & 856 & $v d P$ \\
\hline B & MBE & $\begin{array}{l}20 \mathrm{~nm} \quad \mathrm{Al}_{0.2} \mathrm{Ga}_{0.8} \mathrm{~N} / \\
2 \mathrm{~nm} \mathrm{GaN}\end{array}$ & $12 \%$ & 1101 & $\mathrm{vdP}$ \\
\hline C & MOVPE & $\begin{array}{l}29 \mathrm{~nm} \mathrm{Al}_{0.15} \mathrm{Ga}_{0.85} \mathrm{~N} \\
\text { (with } \mathrm{AlN} \text { interlayer) }\end{array}$ & $5 \%$ & 2093 & HallBar \\
\hline D & MOVPE & $\begin{array}{l}23 \mathrm{~nm} \mathrm{Al}_{0.23} \mathrm{Ga}_{0.77} \mathrm{~N} \\
\text { (with } \mathrm{AlN} \text { interlayer) }\end{array}$ & $4 \%$ & 2254 & HallBar \\
\hline$E$ & MOVPE & $\begin{array}{l}22 \mathrm{~nm} \mathrm{Al}_{0.29} \mathrm{Ga}_{0.71} \mathrm{~N} \\
\text { (with } \mathrm{AlN} \text { interlayer) }\end{array}$ & $2 \%$ & 2230 & HallBar \\
\hline
\end{tabular}

Table 5.1: Summary of different samples with parameters: growth method, structure, experimental sensitivity, mobiltiy at room temperature as determined in van der Pauw geometry (vdP) or Hall bar geometry. The mobilities measured by the two geometries are not directly comparable.

currents of $0.1 \mathrm{~mA}$. All measurements were performed under constant light conditions. The GaN/AlGaN heterostructures were exposed to different $\mathrm{KOH}$ solutions. Standard cleaning was applied to the GaN surface (acetone, 2propanol, de-ionised water and N2 blow-dry) after device fabrication. After each exposure to ionic solution, the samples were soaked in $\mathrm{HCl}$ for $10 \mathrm{~s}$ followed by rinsing in de-ionised water.

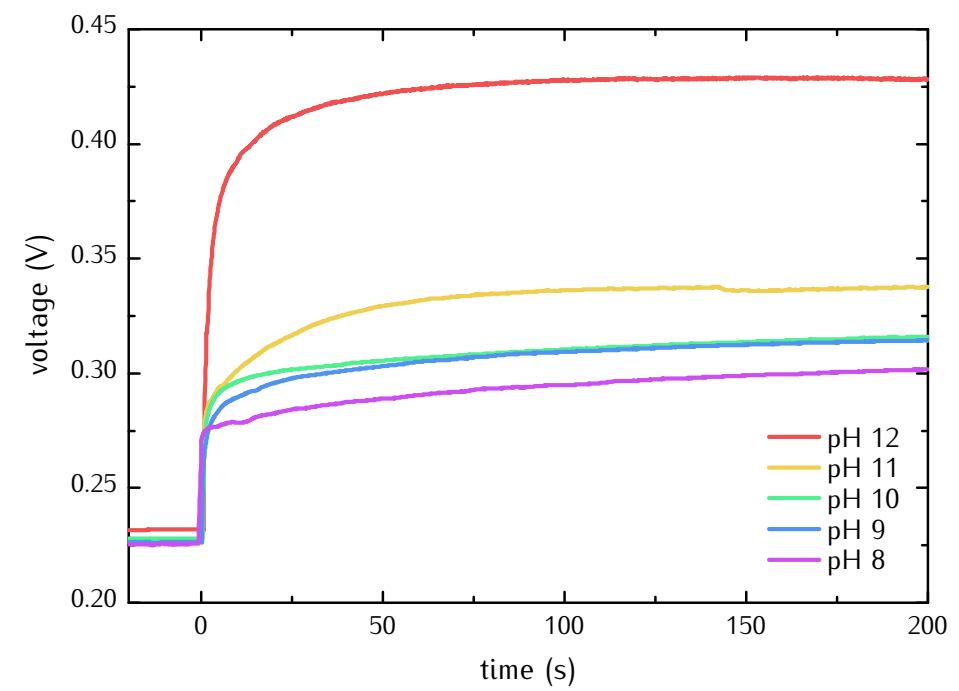

Figure 5.20: Response to $\mathrm{KOH}$ solutions of sample $\mathrm{A}$ measured as a change in voltage for a fixed current. For each voltage curve, $t=0 \mathrm{~s}$ corresponds to the point at which the $\mathrm{KOH}$ solution was applied. Higher responses are observed for higher $\mathrm{pH}$ solutions. 
In an early stage, devices were grown on standard templates which are semi-insulating. These structures showed a low signal to noise ratio. This results from conduction within the bulk GaN in addition to the 2DEG (see Section 3.3). The effect was overcome by growth on insulating Fe:GaN substrates. Two of the sensitivity optimized MBE grown samples are presented here and compared to three MOVPE grown samples from an earlier work which were optimized for high mobility (Table 5.1).

Figure 5.20 shows as-measured time dependent voltage data for an optimized $\mathrm{MBE}$ structure. $\mathrm{A}$ high signal to noise ratio and a strong increase in voltage were observed.

The normalized strength of the response to different $\mathrm{KOH}$ solutions for the five ungated GaN/AlGaN structures can be seen in Figure 5.21. All measured data was normalized to the maximum potential drop that was observed for $\mathrm{KOH} \mathrm{pH} 12$ for each structure. Significant improvements in differentiation between different $\mathrm{pH}$ solutions are achieved for the sensitivity optimized structure grown in this work compared to the MOVPE grown samples. This shows the that by optimization of the heterostructure design, the sensitivity of GaN/AlGaN heterostructure-based ion sensors can be dramatically improved.

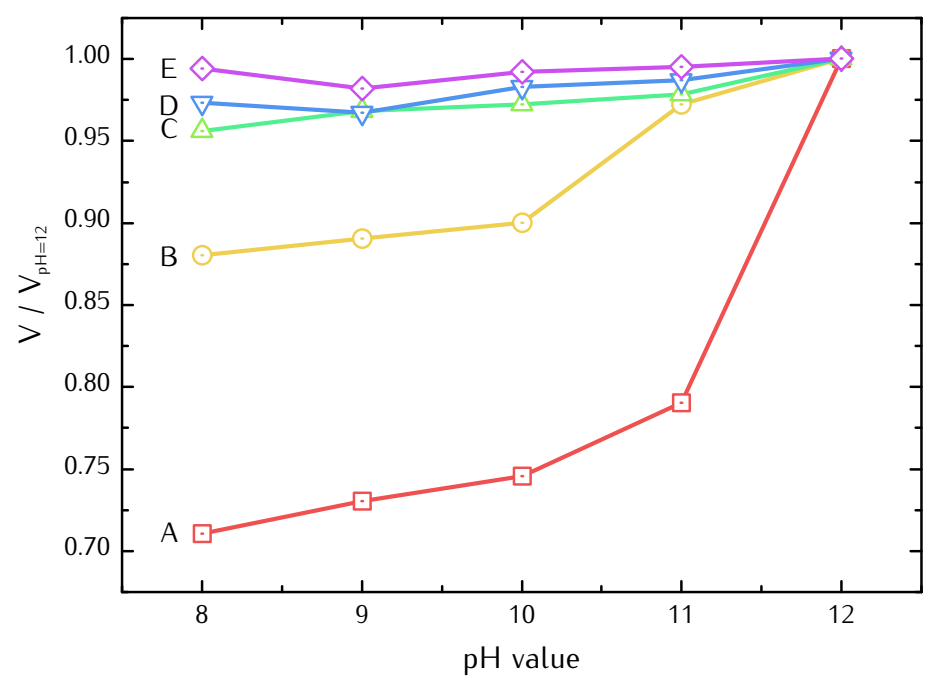

Figure 5.21: Maximum potential drop for five ungated GaN/AlGaN structures exposed to different $\mathrm{KOH}$ solutions (normalized to the value obtained for $\mathrm{pH} 12)$. MBE grown samples $\mathrm{A}$ and $\mathrm{B}$ show the highest response. 



\section{INDIUM GALLIUM NITRIDE HETEROSTRUCTURES}

The optimization process of InGaN growth in this work can be divided into three parts. Early samples have been grown at low temperatures as most results reported in literature. Growth under In bilayer stabilized conditions at higher temperatures with sufficient indium desorption produced better structural quality. The suppression of the observed surface degradation induced by indium and nitrogen resulted in smooth surfaces. This chapter explains the optimization process and its effects on structural and electrical properties in detail.

\subsection{GROWTH CONDITIONS AND STRUCTURAL QUALITY}

The aim of this work was the growth optimization of InGaN heterostructures with respect to low.temperature 2DEG mobility. In GaN/InGaN/GaN 2DEG structures, the electrons are mainly confined within the InGaN layer. This is in contrast to AlGaN based structures, where the 2DEG is mainly confined within GaN. Consequently, the growth optimization of InGaN has a significant impact on the mobility in InGaN heterostructures, just as the optimization of GaN had for GaN/AlGaN structures.

Gallium bilayer stabilized conditions cannot be applied for InGaN growth. Because of the higher bond strength of Ga-N compared to In- $\mathrm{N}$, the incorporation of gallium is energetically favored over that of indium. Therefore, the indium content in InGaN layers is determined by $x_{\ln }=1-\mathrm{Ga} / \mathrm{N}$. Consequently, GaN grows under Ga-rich conditions and no indium incorporates. Growth under overall $\mathrm{N}$-rich conditions, however, resulted in rough surface morphologies. The growth conditions for InGaN layers presented in the following are thus In-rich with Ga fluxes below the GaN stoichiometry, i.e $\mathrm{Ga}<\mathrm{N}$ and $\mathrm{Ga}+\mathrm{In}>\mathrm{N}$. The nitrogen conditions were chosen equal to the optimum GaN growth conditions with an excitation power of $300 \mathrm{~W}$ and a nitrogen flow rate of $0.5 \mathrm{sccm}$. 
Figure 6.1

Atomic force micrograph of a InGaN (0001) surface exemplary for growth at low substrate temperatures. No atomically flat features were observed for growth without stable indium bilayer conditions.

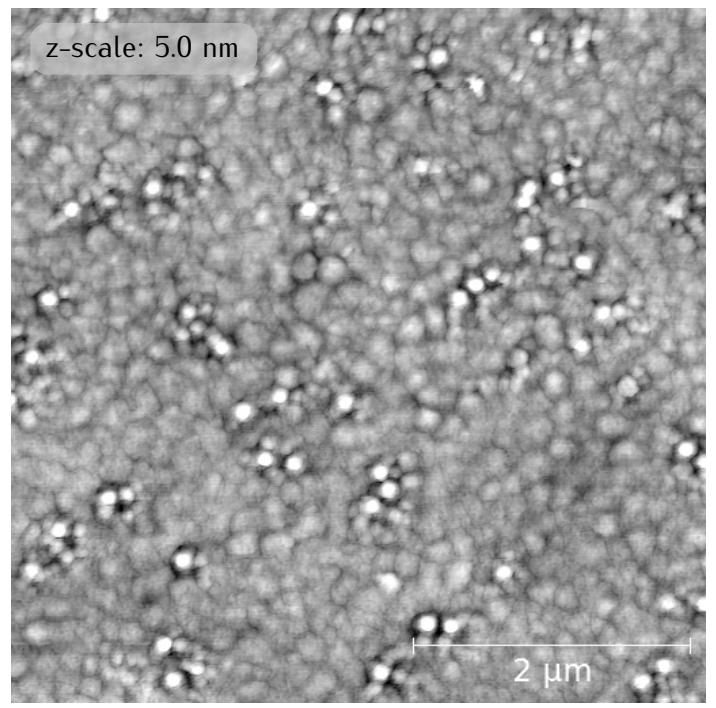

In an early stage of optimization, samples were grown at low substrate temperatures according to growth conditions most commonly reported in the literature as described in Section 1.5.4. This implied growth at substrate temperatures around $T_{s}=500{ }^{\circ} \mathrm{C}$, which is slightly above the decomposition temperature of $\ln N$. In this temperature range, the desorption rate of indium is small compared to the growth rate. Growth under In-rich conditions thus causes the formation of indium droplets. Therefore, the In-flux was chosen only slightly above the stoichiometry in order to keep the amount of accumulated indium low.

Several series of structures were grown at low temperatures with varying parameters. The gallium supply was varied from low fluxes up to almost stoichiometric conditions. Indium was supplied up to overall metal rich conditions, well above the onset of droplet formation $(\mathrm{GaN}+\ln =2)$. The
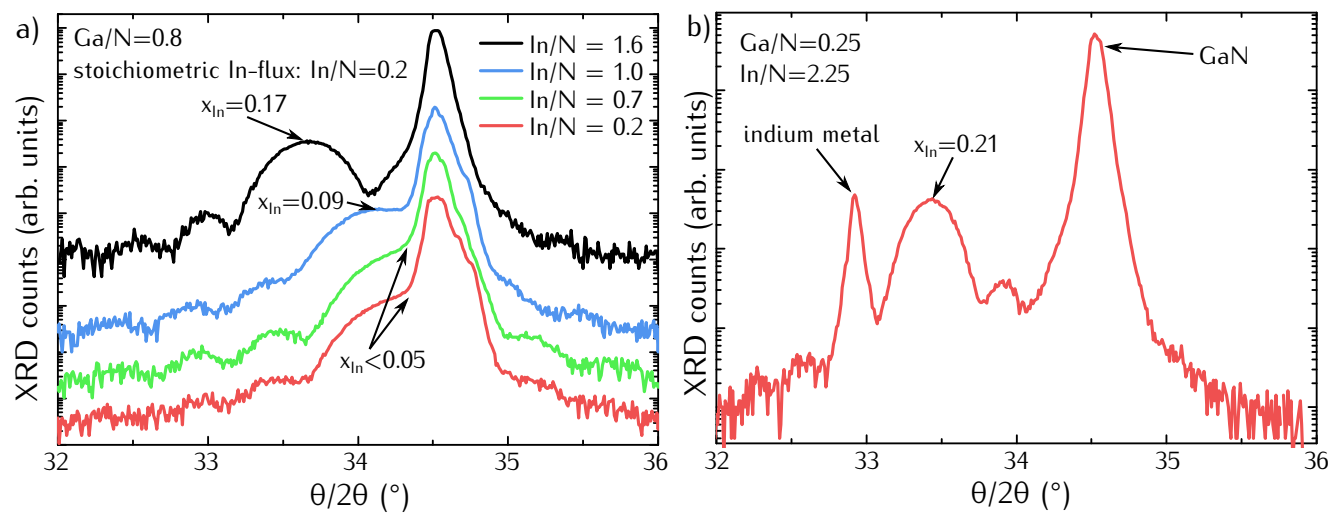

Figure 6.2: $\mathrm{XRD} \theta / 2 \theta$ scans of InGaN heterostructures grown under indium bilayer stabilized conditions at $T_{S}=620^{\circ} \mathrm{C}$. a) $x_{\ln }$ increases with indium supply, reaching a maximum at high indium fluxes. b) indium metal peaks are found for growth with very low $\mathrm{Ga} / \mathrm{N}$ ratios which coincides with a reduced growth rate. 
substrate temperature was varied from $T_{S}=400{ }^{\circ} \mathrm{C}$ to $T_{S}=550{ }^{\circ} \mathrm{C}$, above which no indium incorporation was observed for the applied indium fluxes. All InGaN structures grown in this parameter range showed rough surface morphologies. One example is shown in Figure 6.1. Furthermore, no 2DEGs were measured in GaN/InGaN/GaN heterostructures.

Growth under indium bilayer stabilized conditions promised better surface morphologies. Stable indium bilayers form for sufficient indium desorption. This was achieved for substrate temperatures above $570{ }^{\circ} \mathrm{C}$. In this temperature range, the indium incorporation not only to depends on $T_{s}$ and $\mathrm{Ga} / \mathrm{N}$ but also on the indium flux. Figure 6.2 a) shows XRD $\theta / 2 \theta$ scans for structures grown at $T_{s}=620^{\circ} \mathrm{C}$ with different In supplies. The indium content $x_{\ln }$ was determined according to the literature [121]. It is observed, that $x_{I n}$ increases with the indium supply, with very little indium incorporation under stoichiometric conditions. For growth under highly indium rich conditions, the indium content reached a maximum $x_{I n}^{\max }$, which depends on the substrate temperature (see Section 6.3). No InGaN growth was observed above $T_{s}=650^{\circ} \mathrm{C}$.

The impact of the Ga supply on InGaN layers grown under In-rich, high temperature growth conditions depends on $x_{l n}^{\max }$. At substrate temperatures below $T_{s}=500{ }^{\circ} \mathrm{C}$, the indium content is determined by the Ga supply with $x_{I_{n}}=1-\mathrm{Ga} / \mathrm{N}$ and increases as the Ga flux decreases. Growth at higher temperatures limits the indium content and $x_{/ n}$ does not increase with decreasing Ga flux if $1-\mathrm{Ga} / \mathrm{N}<x_{/ n-m a x}$. Under these conditions the growth rate decreases if the Ga-flux decreases (see Section 6.3). For very low

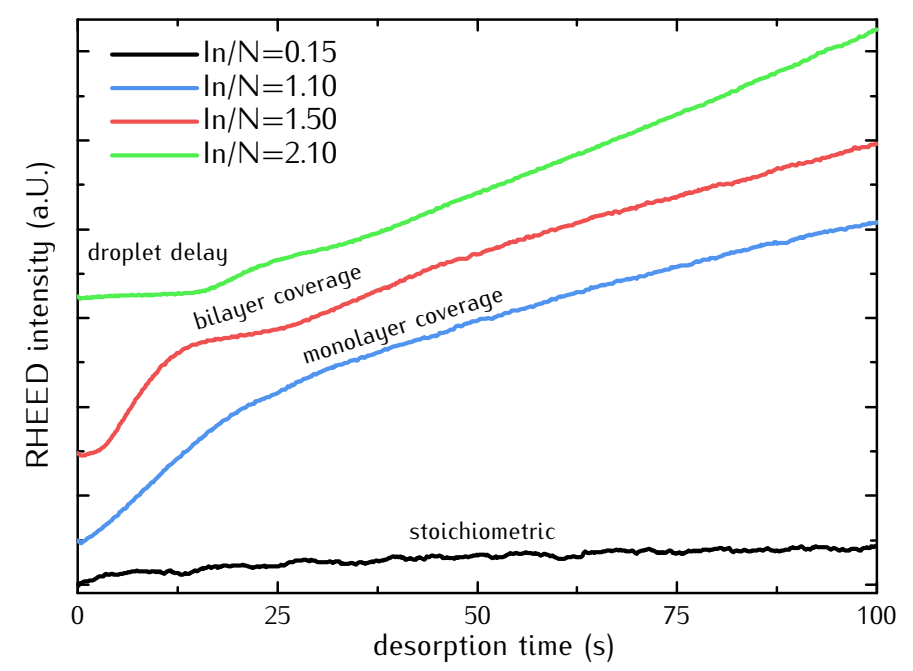

Figure 6.3: RHEED intensity during indium desorption at $620^{\circ} \mathrm{C}$ after $\operatorname{lnGaN}$ growth with different In-fluxes. Four different characteristic trends are observed: no increase - stoichiometric, continuous increase - monolayer coverage, oscillation - bilayer coverage, delayed oscillation - droplet coverage. 
Ga-fluxes, the formation of metallic In clusters was observed in XRD as shown in Figure 6.2. Therefore, the gallium supply was chosen close to $1-\mathrm{Ga} / \mathrm{N}=x_{\ln }^{\max }$ in the following.

The desorption of indium was studied by RHEED in order to investigate the indium coverage for varying indium flux. Characteristic trends of the RHEED intensity for different coverages were observed as shown in Figure 6.3. They were used to identify the transitions between droplet, bilayer, monolayer and stoichiometric growth depending on the indium flux.

The determined transitions over indium flux and substrate temperature for a gallium supply of $\mathrm{Ga} / \mathrm{N}=0.8$ are shown in Figure 6.4. A window for indium bilayer stabilized growth of $\ln G a N$ with concentration up to $x_{\ln }=0.3$ is available over a wide temperature range. Note, that the indium contents given in this figure are only a guide to the eye as the dependence of $x_{\ln }$ on the indium supply was not studied at various temperatures. The iso-contents lines were estimated based on the incorporation at low temperatures and on the maximum indium content under highly In-rich conditions (see Section 6.3).

All structures grown under indium bilayer stabilized conditions (in combination with the procedure described in Section 6.2) showed a good overall quality. Atomic force micrographs as shown in Figure 6.5 indicated a spiral growth mode. Atomically flat terraces are observed, and the morphology is

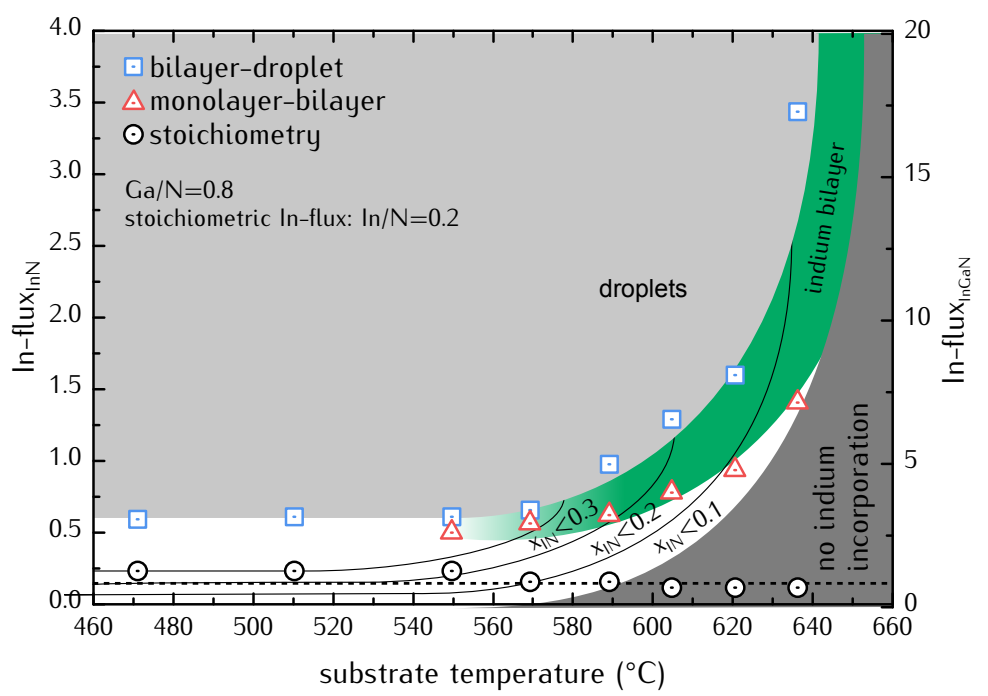

Figure 6.4: Diagram of the indium coverage depending on substrate temperature and indium flux as determined by RHEED for $\mathrm{Ga} / \mathrm{N}=0.8$. The indium flux is given normalized to the $\ln N$ stoichiometry (left) and to the $\ln G a N$ stoichiometry (right). Indium bilayer stabilized growth is possible above $570{ }^{\circ} \mathrm{C}$. Note that the indium supply in this range is highly In-rich compared to the InGaN stoichiometry. The indium content was estimated based on the low temperature incorporation and on the incorporation under high In supply. 


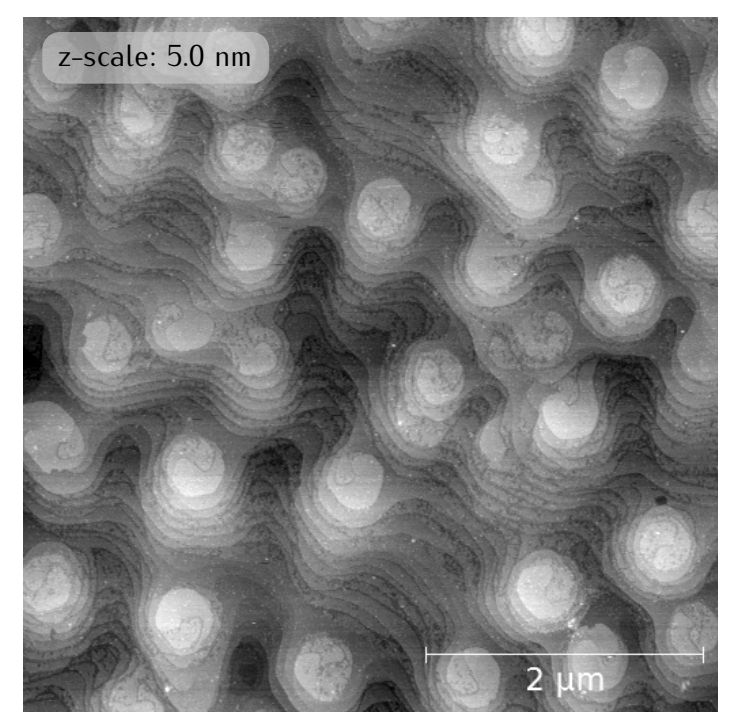

Figure 6.5

Atomic force micrograph of sample G1061 grown under indium bilayer stabilized conditions with excess Ga. The spiral growth mode with atomically flat terraces is observed.

comparable to that GaN and AlGaN layers.

For the growth of $\mathrm{GaN}$ in GaN/InGaN/GaN heterostructures, the optimized GaN growth conditions were applied. Therefore, the growth temperature was lowered during InGaN growth and increased to $700{ }^{\circ} \mathrm{C}$ for the growth of the GaN cap layer. The impact of the whole growth procedure on the InGaN layer was investigated by transmission electron micrographs and energy dispersive X-ray. The results are shown in Figure 6.6. No additional dislocations formed at InGaN interfaces, and no segregation was found. The analysis of an InGaN/AlGaN structure showed no interdiffusion of indium. This could have resulted from the increased temperature and would decrease the sharpness of the interface. The TEM measurements, however, revealed
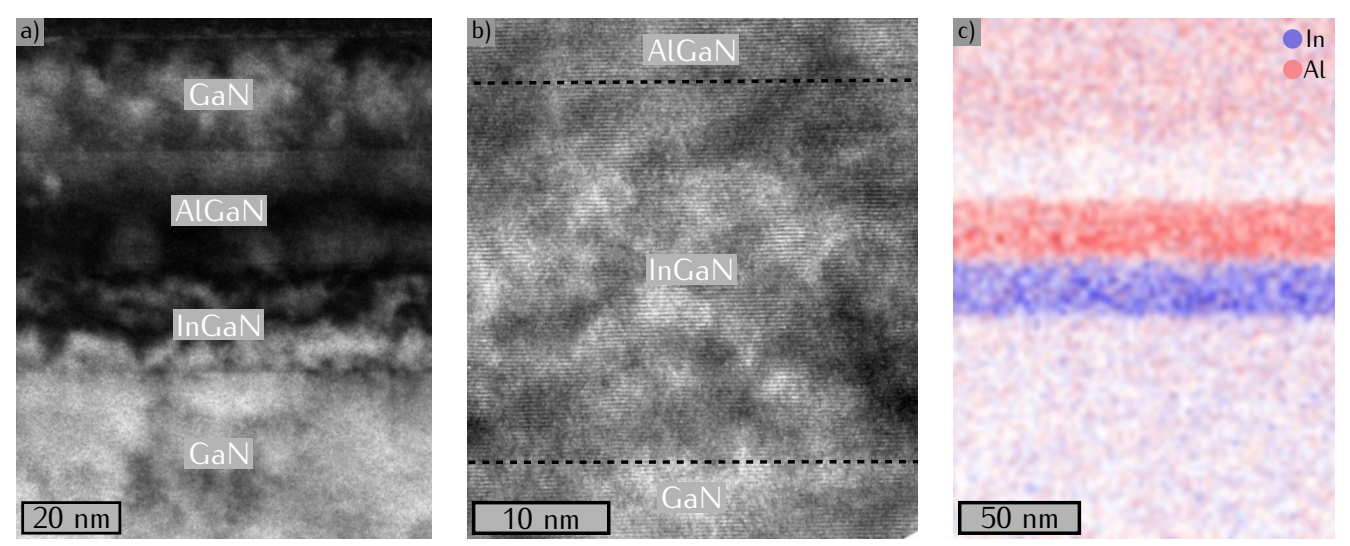

Figure 6.6: a) and b) Transmission electron micrographs of an GaN/InGaN/AlGaN/GaN sample (ID: G1086). Sharp interfaces with no additional dislocations are observed. Inhomogeneities are observed in the InGaN layer, which are likely the result of fluctuation in $x_{/ n}$. c) TEM EDX of G1086. No diffusion of indium into the AlGaN layer and no In-rich clusters are observed. 
strong inhomogeneities within the InGaN layer. They are likely the results of fluctuations in the indium concentration as reported in the literature [135].

\subsection{SURFACE DEGRADATION AFTER INDIUM DESORPTION}

For high mobilities in 2DEGs, the interface quality in heterostructures is of immense importance. The growth under indium bilayer stabilized conditions showed a spiral growth mode. However, another step had to be applied in order to achieve smooth InGaN surfaces.

All samples grown in the In bilayer stabilized regime showed a degraded surface after indium desorption. The observation of pits with a diameter of $2 \mathrm{~nm}$ on InGaN (0001) surfaces for indium rich growth conditions has been reported before [64]. The impact on the surface morphology observed in this work is however much stronger. An example is shown in Figure 6.7 a) exhibiting cracks and pits up to $3 \mathrm{~nm}$ deep. These defects act as scattering centers for electron transport, making the observed morphologies fatal for the mobility in 2DEGs.

Some observations suggested that the degradation of the surface is a post growth process. Some structures showed spiral hillocks with atomically flat terraces on an otherwise degraded surface (see Figure 6.7 b)). On one samples a missing link was found (see Figure $6.7 \mathrm{c}$ )). This spiral hillock showed atomically smooth terraces at the base changing to pitted terraces further up. If the pits would form during growth, this should not be observed. This suggests that the surface degrades after growth.

Possible processes are oxidation or rough crystallization of a metallic adlayer during cooldown. This was studied by treating the samples with $\mathrm{HCL}$, which removes metals, and with $\mathrm{KOH}$, which etches oxides. No change in the morphology was found. As nitrides withstand both treatments, a reaction with nitrogen or surface segregation are a plausible explanation for the degradation.
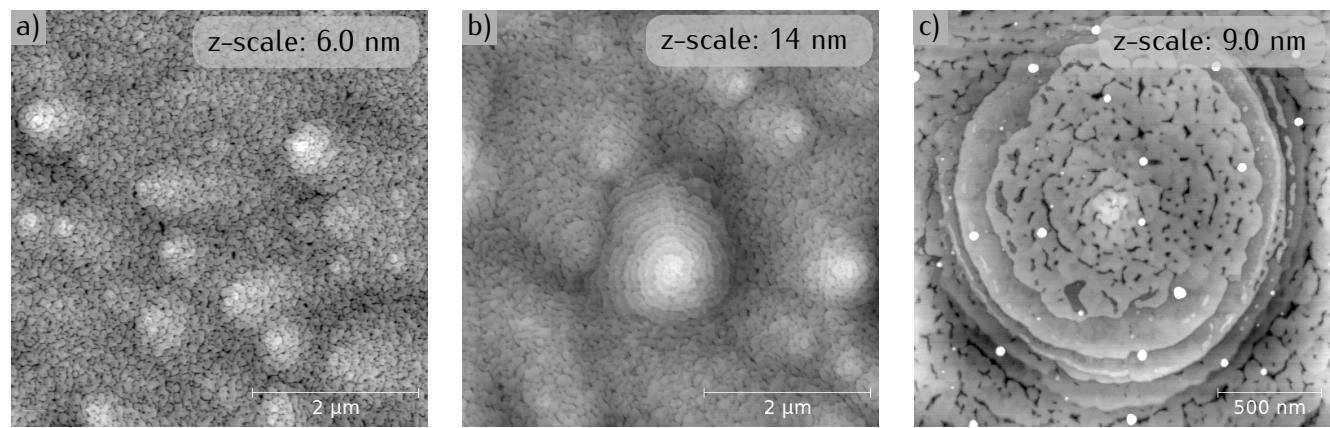

Figure 6.7: Atomic force micrograph of the $\operatorname{lnGaN}(0001)$ surface showing a) a degraded surface, b) a spiral on an otherwise degraded surface and c) missing link - a spiral which is smooth at the base but degraded at the top. The observations suggest a post growth degradation. 

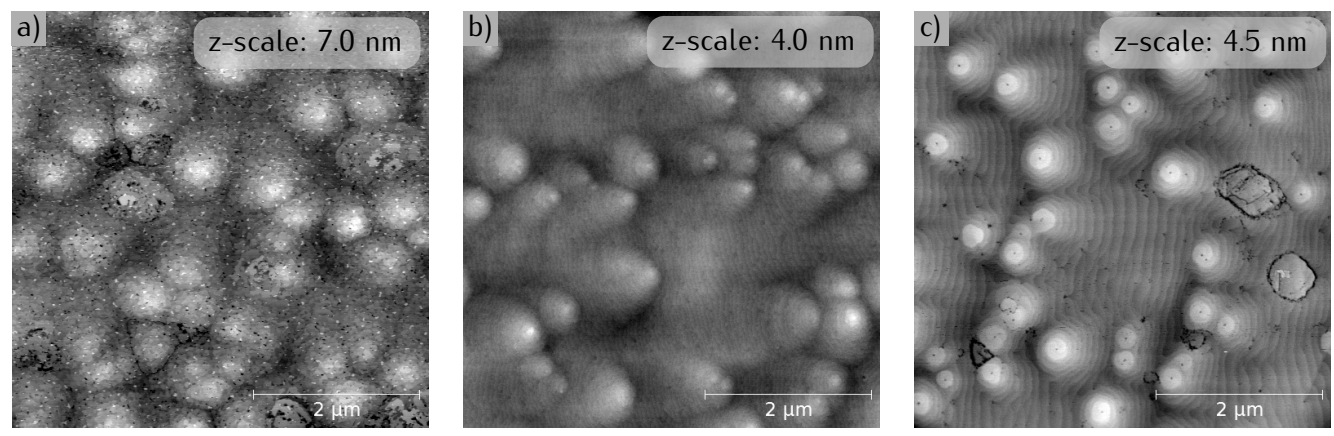

Figure 6.8: Atomic force micrographs of the $\operatorname{lnGaN}(0001)$ surface a) after indium desorption under UHV conditions, b) no indium desorption and c) indium desorption under excess gallium, which resulted in the best morphology. For b) and c) the metals were removed by $\mathrm{HCL}$ after growth.

This presumption was investigated by applying three different treatments after InGaN growth: 1) indium desorption without supplying nitrogen to the growth chamber (UHV: $p_{\text {base }}=5 \times 10^{-10}$ mbar) 2) no indium desorption with excess In during cool down, 3) excess Ga during In desorption and cool down. The results are shown in Figure 6.8. The morphology for UHV desorption showed only a minor improvement compared to regular desorption. The other two steps, however, resulted in pit free surfaces. The degradation therefore occurs if indium desorbs directly from the surface into an ambient nitrogen atmosphere leaving a bare InGaN surface. The best morphology was obtained for excess Ga.

By applying excess Ga before InGaN growth, the number of threading dislocations was significantly reduced. This is shown in Figure 6.9 where two samples with and without excess Ga before InGaN growth are compared. The black spots originate from edge dislocations penetrating the surface.
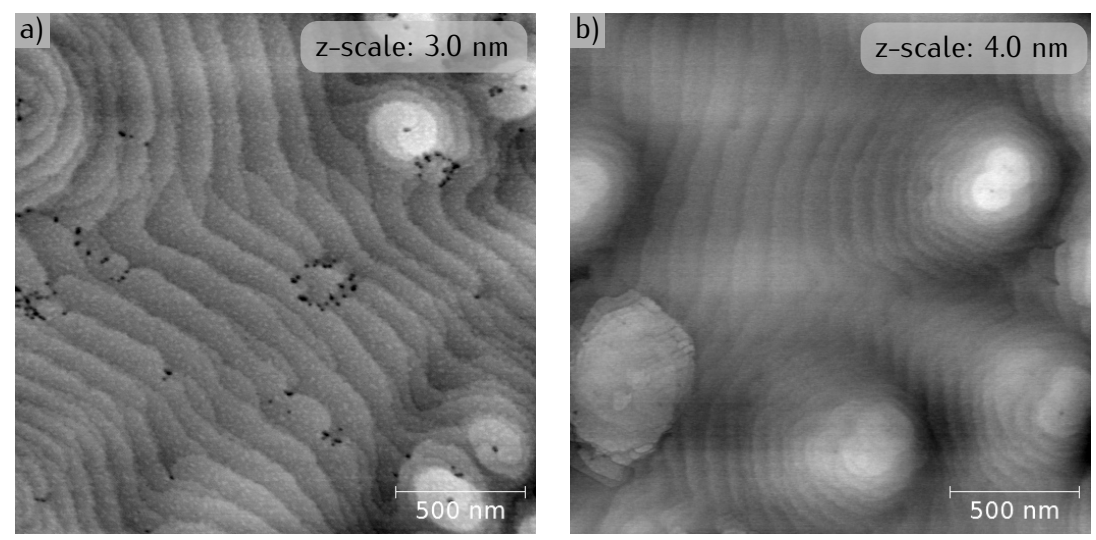

Figure 6.9: Atomic force micrographs of the $\ln G a N(0001)$ surface with excess Ga a) only after InGaN growth and b) before and after InGaN growth. Excess In before $\ln \mathrm{GaN}$ growth was applied in a). The number of dislocations (black spots in a)) was reduced by excess Ga before InGaN growth. 

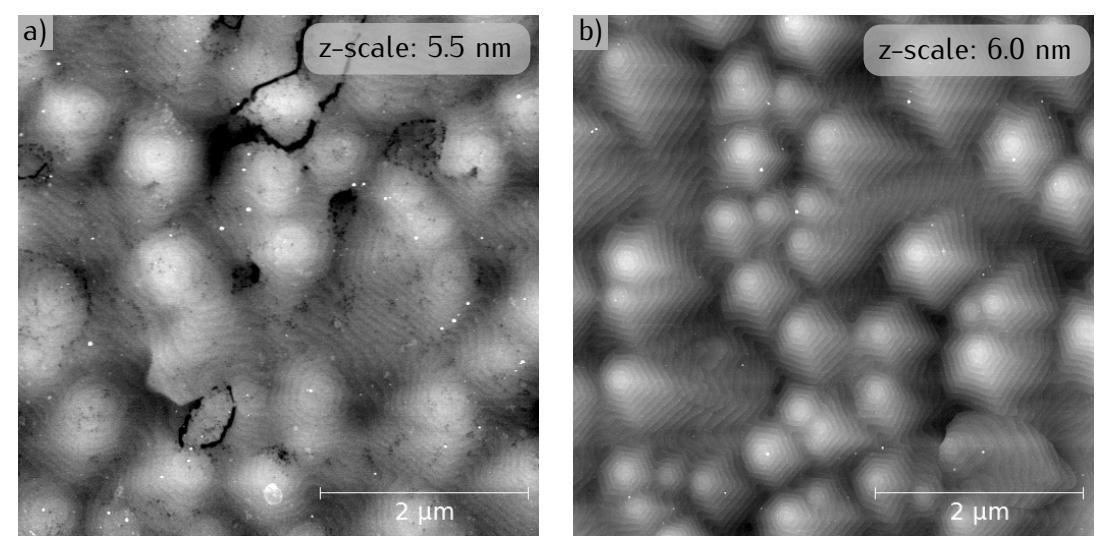

Figure 6.10: Atomic force micrographs of the InGaN (0001) surface of GaN/InGaN/$\mathrm{GaN}$ heterostructures with a) complete desorption before GaN cap layer growth and b) only indium desorption with excess Ga remaining on the surface before GaN cap layer growth. A lower defect density is achieved for no metal desorption before cap layer growth.

This observation cannot be explained by surface segregation. In this process, incorporated In atoms leave an InGaN layer at high temperatures due to the weak In-N bond. However, the dislocation reduction was observed when excess Ga was applied before InGaN growth, when no InGaN has grown yet. Surface segregation during the beginning of InGaN growth can also be excluded. Because the excess Ga reduced the dislocation generation and the surface degradation, it is reasonable to assume that both processes have the same origin. The sample shown in Figure 6.9 a) was, however, with excess In before InGaN growth. Excess In also reduced the surface degradation after growth, and should thus also suppress the dislocation generation if surface segregation has a negative effect on InGaN growth. Both processes were only observed if thin In adlayers were in direct contact with the ambient nitrogen, and it is therefore assumed that their origin is the reaction of indium with nitrogen.

The impact of excess Ga on the growth of GaN caplayers was also studied. Figure 6.10 a) shows the surface of a GaN/InGaN/GaN heterostructure. For this sample, all metals desorped before GaN caplayer growth. Figure 6.10

Figure 6.11

RHEED-[1ํㅣ after indium desorption from the degraded InGaN (0001) surface. A $\sqrt{3} \times \sqrt{3} R 30^{\circ}$ is clearly observed.

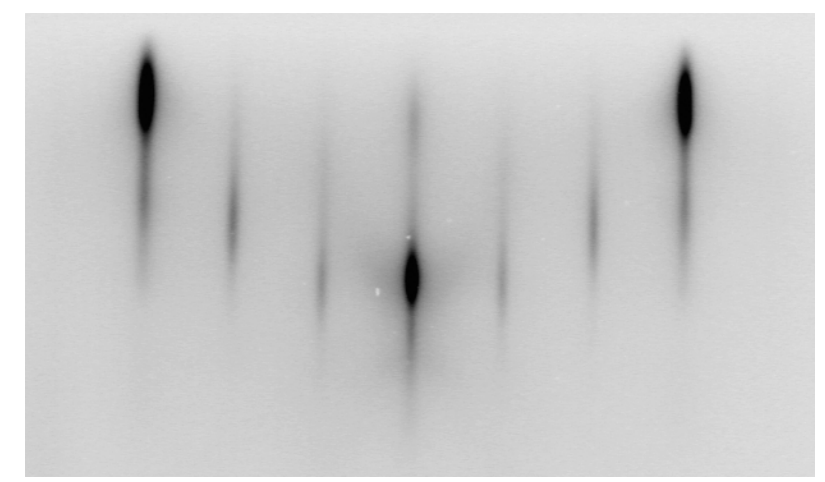


b) shows the surface of a similar structure, where only indium desorped after InGaN growth, leaving excess Ga on the surface. Atomically flat terraces are observed for both samples. However, the desorption of all metals results in many defects. The optimum growth of InGaN heterostructures was therefore carried out under indium bilayer stabilized conditions with excess Ga during growth interruptions before and after InGaN growth.

During the optimization process presented in the previous paragraphs it was found, that a $\sqrt{3} \times \sqrt{3} R 30^{\circ}$ RHEED reconstruction coincided with the surface degradation. This reconstruction, which is shown in Figure 6.11, always appeared after indium desorption if no excess Ga was applied. No reconstruction was observed after metal desorption, if first indium desorpted and then gallium. This compares well with the observation of the reconstruction reported in the literature [64]. The reconstruction forms under nitrogen atmosphere on GaN (0001) or InGaN (0001) surfaces covered with $1 / 3 \mathrm{ML}$ of indium. It is not observed after indium desorption if $\mathrm{In}$ is deposited onto a gallium adlayer. This supports the presumption that the degradation arises from a reaction of a thin indium layer with the ambient nitrogen. The authors also analyzed the growth depending on Ga and In supply. Above a certain indium flux they obtained smooth surfaces. During growth with this flux, they observed a $\sqrt{3} \times \sqrt{3} \mathrm{R} 30^{\circ}$ reconstruction by RHEED. The reconstruction was therefore linked to the growth of smooth layers. In this work the reconstruction rather indicated the degradation of a surface that was smooth beforehand.

The requirement of excess Ga during the entire growth process complicates

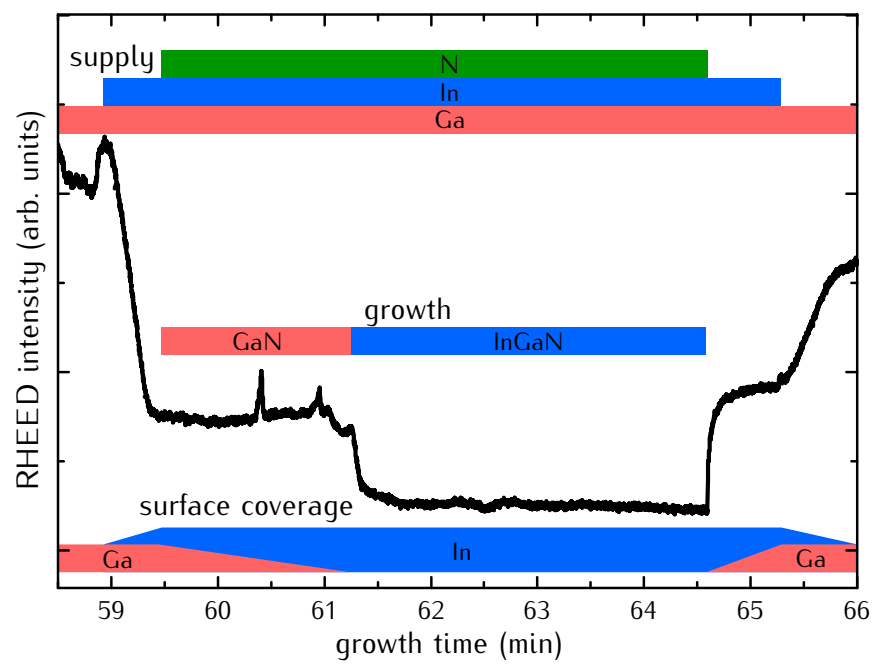

Figure 6.12: RHEED intensity during InGaN growth with excess $\mathrm{Ga}$ before and after InGaN growth. The supply, the growing material and the surface coverage are schematically indicated. The effective growth time is much shorter than the In+Ga+N supply. InGaN grows only after all excess Ga incorporated, which is observed by a minimum in the RHEED intensity. 
the control of the InGaN thickness. InGaN grows only if there is no Ga on the surface, because $\mathrm{Ga}$ is preferably incorporated over $\mathrm{In}$. The beginning of InGaN growth can however be monitored by RHEED. The RHEED intensity is reduced by the presence of liquid metallic layers. The intensity drop is material dependent since the scattering cross section of $\mathrm{Ga}$ is higher compared to In. The RHEED intensity is therefore different for surfaces cover with Ga or In or both.

A typical RHEED intensity signal during growth is shown in Figure 6.12. At the beginning, the surface is covered with gallium. If the In, Ga and N shutters are opened, the intensity drops. During this time In accumulates and excess $\mathrm{Ga}$ incorporates because $\mathrm{Ga} / \mathrm{N}<1$. At some point, no $\mathrm{Ga}$ is left on the surface and InGaN starts growing under a thick In wetting layer. This is indicated by another decrease in the RHEED intensity.

\subsection{INDIUM INCORPORATION DETERMINED BY RHEED}

The indium content in InGaN layers grown under indium bilayer conditions depends on the indium flux, on 1-Ga/ $\mathrm{N}$ and the substrate temperature $T_{s}$. At a given $T_{s}$, there exists a maximal incorporable amount of indium $x_{l n}^{\max }$ for growth under highly indium-rich conditions. In this work a novel method was developed to estimate $x_{\ln }^{\max }$ by a RHEED analysis. The idea of this method is that $x_{l n}^{\max }$ corresponds to one gallium flux Gamax with $x_{l n}^{\max }=1-\mathrm{Ga}_{\max } / \mathrm{N}$ as illustrated in Figure 6.13.

This flux is determined by a study of the incorporation rate of indium over Gaflux for a high indium supply. For GaN growth, the growth rate decreases

Figure 6.13

Illustration of how $\mathrm{Ga}_{\max }$ and thus $x_{l n}^{\max }$ are determined from $\tau$. The illustration implies a high indium supply and indium incorporation only below $T_{0}$. a) Growth rate over Ga-flux: For Ga-fluxes smaller than GaN stoichiometry, the In content increases and growth rate is constant for decreasing Ga-flux. For Ga-fluxes smaller than $\mathrm{Ga}_{\max }$, the In content is constant and growth rate decreases for decreasing Ga-flux. b) Resulting indium incorporation rate over Ga-flux. c) $\tau$ observed by RHEED. $\tau$ is shortest for $\mathrm{Ga}_{\max }$.

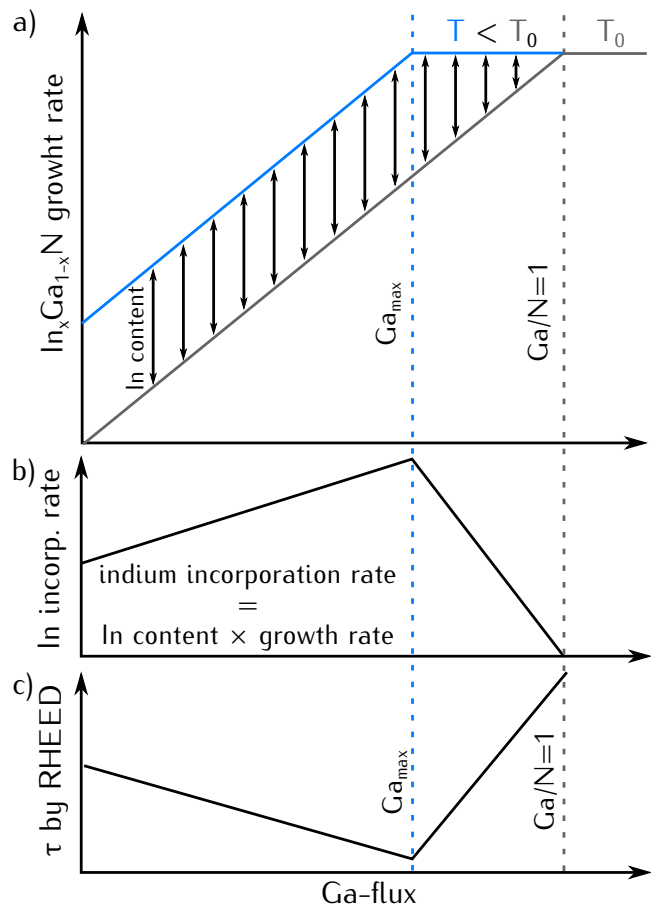



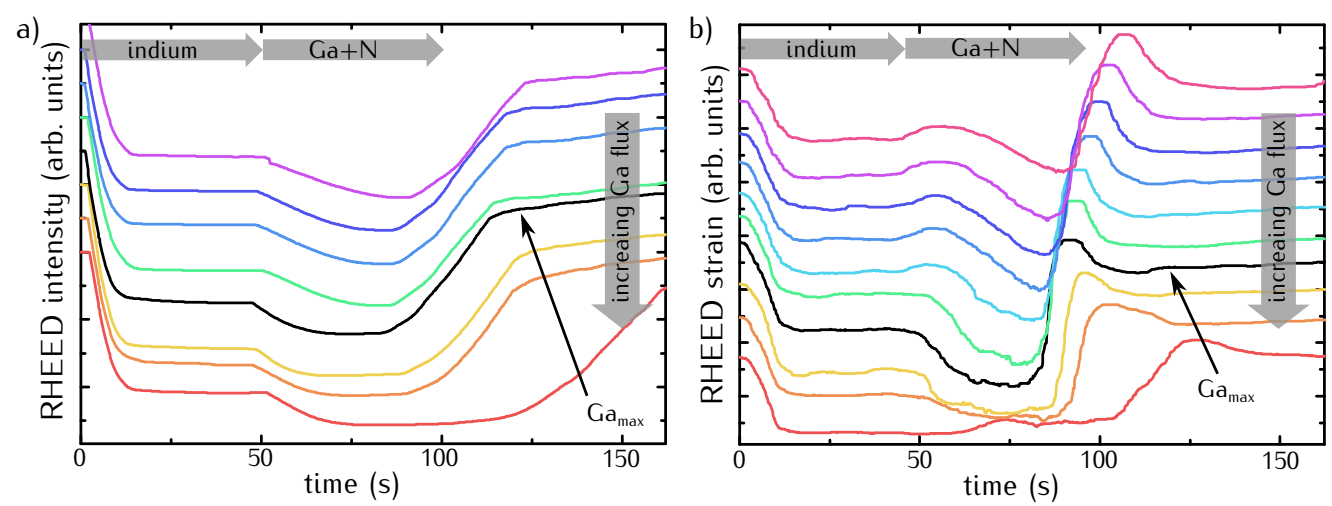

Figure 6.14: Incorporation analysis for varying Ga-flux by a) RHEED intensity and b) RHEED strain. Indium supplied between $0 \mathrm{~s}$ and $50 \mathrm{~s}$. Ga $+\mathrm{N}$ supplied $>50 \mathrm{~s}$. The Ga-flux resulting in the shortest $\tau$ is Gamax.

below the stoichiometry at $\mathrm{Ga} / \mathrm{N}=1$. If no indium incorporates at high temperatures this is true even if indium is supplied to the growing surface (gray line in Figure 6.13 a)). At temperatures where indium incorporates, the growth rate does not decrease right below $\mathrm{Ga} / \mathrm{N}=1$ as the missing $\mathrm{Ga}$ is replaced by In (blue line in Figure 6.13 a)). Here, the indium content increases with decreasing Ga-flux. If the maximum indium content incorporates, a further decrease of the Ga-flux results in a reduced growth rate. The incorporation rate of indium depends on the growth rate and the indium content (Figure 6.13 b)). At a constant growth rate is increases with the indium content. For a fixed content, it reduces with the growth rate. Therefore, the incorporation rate is maximal for growth with $\mathrm{Ga}_{\max }$ and a high indium supply.

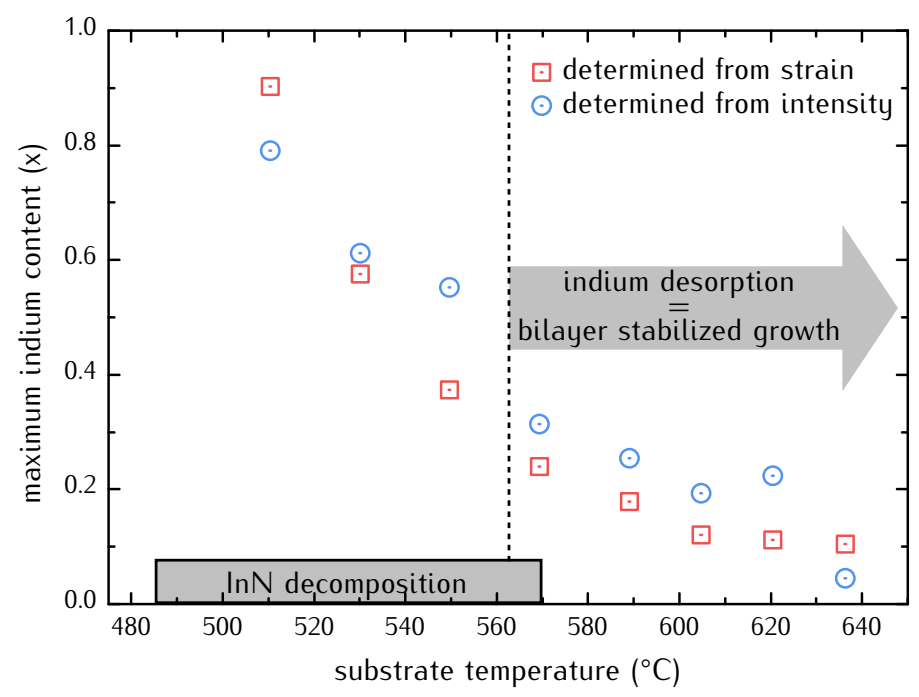

Figure 6.15: Maximum indium content determined by RHEED intensity and RHEED strain over substrate temperature. Growth under indium bilayer stabilized conditions is limited to an $x_{\ln -\max }=0.3$. No indium is incorporated above $650{ }^{\circ} \mathrm{C}$. 
The incorporation rate can be deduced from a RHEED analysis by applying a series of steps. An example of the RHEED intensity and the strain during this process is given in Figure 6.14. Both are sensitive to the metal coverage (see Section 2.1.4), however, only the intensity is addressed in the following. For a fixed substrate temperature, a certain amount of indium was deposited on a GaN surface. This results in a decrease of the RHEED intensity by the accumulated indium. After the indium supply is closed, and gallium and nitrogen are supplied with a Ga-flux below the GaN stoichiometry. Because $\mathrm{Ga} / \mathrm{N}<1$, the excess indium incorporates at a certain rate. $\mathrm{A}$ particular amount of time $\tau$ after the growth was started, no more indium is left on the surface, and the RHEED intensity increases. The incorporation rate of indium is therefore inversely proportional to $\tau$ as illustrated in Figure 6.13 c).

In order to determine $x_{\ln }^{\max }$, the described steps have been applied for varying Ga-fluxes. The Ga-flux that results in the shortest time $\tau$ is Gamax. The maximal incorporable amount of indium was then determined by $x_{l n}^{\max }=1$ $\mathrm{Ga}_{\max } / \mathrm{N}$.

The results of this anlysis for different temperatures are shown in Figure 6.15. A strong temperature dependence of $x_{l n}^{\max }$ is observed. Within the In bilayer stabilized growth regime, InGaN layers with In contents up to $x \ln -\max =0.3$ can be produced. No indium incorporation is possible above $650{ }^{\circ} \mathrm{C}$.

\subsection{HETEROSTRUCTURE DESIGN}

Two dimensional hole gases (2DHG) form in uncapped GaN/InGaN structures (see Section 3.1). The hole mobility in InGaN is however much lower than the electron mobility [90]. Two dimensional electron gases (2DEG) thus
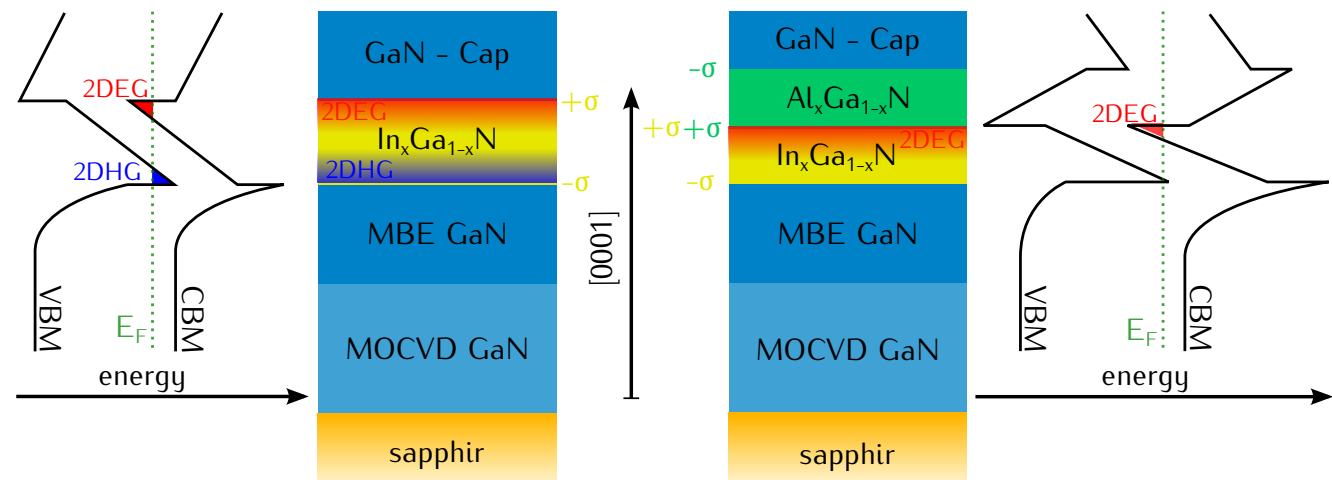

Figure 6.16: Schematic of InGaN and InGaN/AlGaN based heterostructures. The polarization charges $\sigma$ and the band profiles are indicated. In both structures a 2DEG and a 2DHG form within InGaN. Additional polarization charges introduced by an AlGaN layer allow only electron confinement for thin InGaN barriers. 

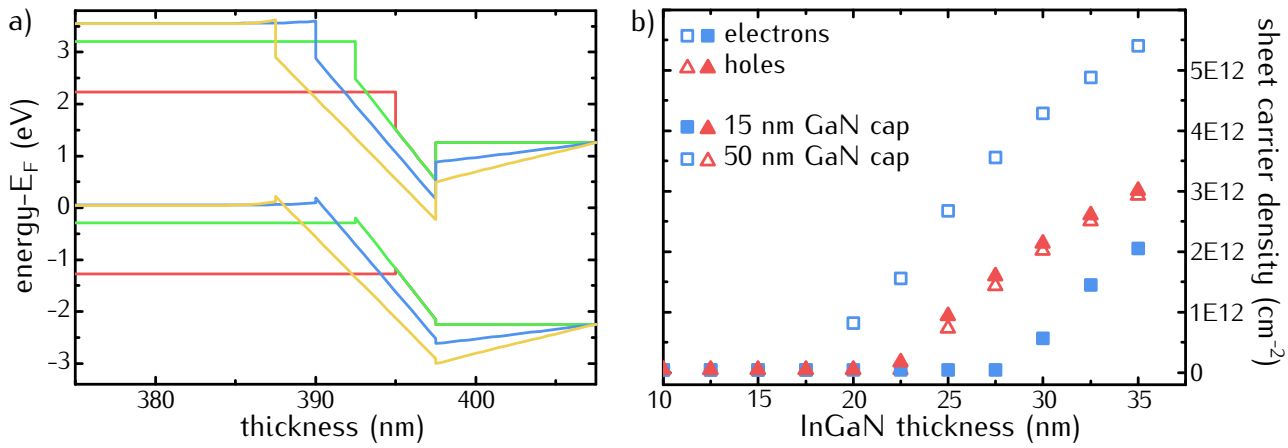

Figure 6.17: a) Profile of CBM and VBM with a $\ln _{0.2} \mathrm{Ga}_{0.8} \mathrm{~N}$ layer with varying thickness, without doping. b) sheet carrier density over $\ln _{0.1} \mathrm{Ga}_{0.9} \mathrm{~N}$ thickness for a structure with a $15 \mathrm{~nm}$ and a structure with a $50 \mathrm{~nm}$ GaN cap layer. $n_{s H}$ and $n_{s E}$ increases comparably with InGaN thickness. For thicker capping layers, however, more electrons than hole can be obtained.

promise better results for low temperature magneto transport measurements. Under certain conditions, 2DEGs form in addition to 2DHGs in GaN/In$\mathrm{GaN} / \mathrm{GaN}$ heterostructures. The coexistence of $\mathrm{p}$ - and $\mathrm{n}$-conductive channels is however not desired as it results in ambiguous transport results (see Section 3.3). This section therefore studies the impact of the heterostructure design on the sheet carrier densities of 2DEGs and 2DHGs.

Two types of InGaN based heterostructures were grown during this work as depicted in Figure 6.16. First GaN/InGaN/GaN and then GaN/InGaN/Al$\mathrm{GaN} / \mathrm{GaN}$ structures will be discussed. For both types of structures, the electron channel lies within the InGaN layer.

In Figure $6.17 \mathrm{a}$ ), the profile of the conduction band minimum (CBM) and the valence band maximum (VBM) in a GaN/InGaN/GaN structures with different InGaN thicknesses is shown. No doping is considered for better illustration. The direction of the polarization field in InGaN results in an upward tilt of the bands with respect to the surface. For thicker layers, the voltage difference between the two interfaces of the InGaN layer increases. Above a critical thickness, VBM becomes larger than $E_{F}$ and a $2 \mathrm{DHG}$ forms with a sheet hole density $n_{S H}$. The critical thickness depends on the strength of the polarization field and thus on the In content.

The accumulated holes represent a charge which induces an additional field between the lower GaN/InGaN interface and the surface. The direction of this field is opposite to the polarization field. It reduces the effective field within InGaN and results in a downward tilt of the bands in the GaN cap layer. The strength of this field depends on $n_{s} H$, and for large $n_{s H}$, the CBM drops below $E_{F}$ at the upper InGaN/GaN interface. Electrons accumulate and form a 2DEG with a sheet electron density $n_{s E}$. The formation of the 2DEG requires sufficiently high $n_{s H}$. Therefore, a 2DEG forms at thicker InGaN barriers compared to the 2DHG. In addition, $n_{s E}<n_{s H}$ for all $\operatorname{lnGaN}$ 

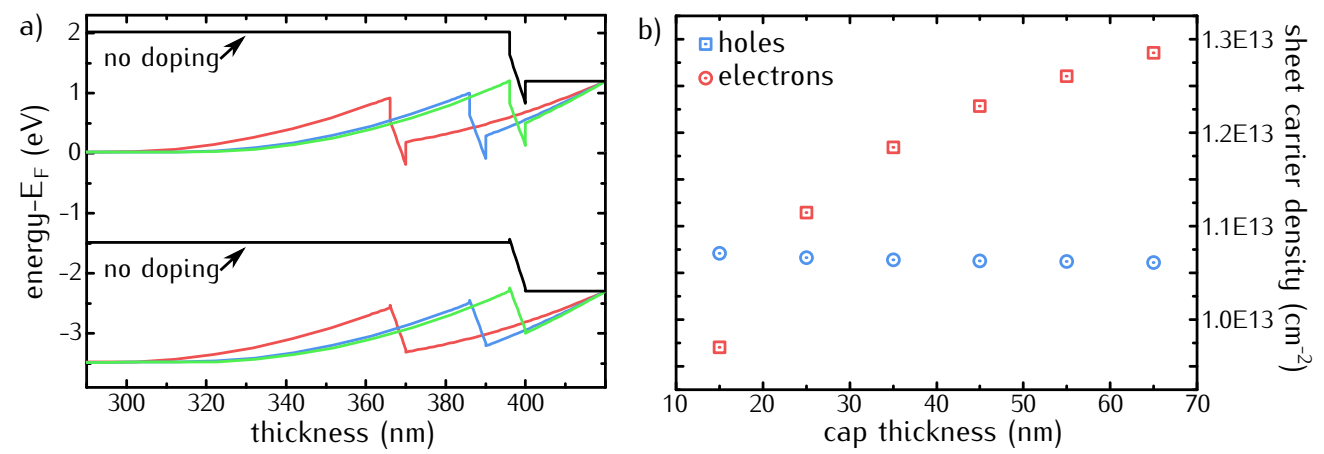

Figure 6.18: a) Profile of $C B M$ and VBM for a $4 \mathrm{~nm} \ln _{0.2} \mathrm{Ga}_{0.8} \mathrm{~N}$ barrier with varying GaN cap layer thickness. An undoped structure is given for comparison. b) Sheet carrier density over GaN cap layer thickness for a $20 \mathrm{~nm}$ $\ln _{0.2} \mathrm{Ga}_{0.8} \mathrm{~N}$ barrier. The electron density increases with cap layer thickness, with the hole density remaining almost unaffected.

layer thicknesses. This is shown for the thin cap layer in Figure 6.17 b), where now doping is taken into account.

The situation changes for thicker GaN cap layers because of screening of the surface potential. As shown in Figure 6.18 a) the screening results in a downward band bending below the surface. For thicker GaN cap layers, CBM drops below $E_{F}$ and a 2DEG forms with no 2DHG existing. Therefore, 2DEGs may form at InGaN thicknesses lower than the critical thickness for 2DHG formation. This is shown in Figure $6.17 \mathrm{~b}$ ) for the thicker cap layer. With increasing cap layer thickness, $n_{S E}$ increases while $n_{S H}$ remains constant as shown in Figure 6.18 b). Thick cap layers are thus needed

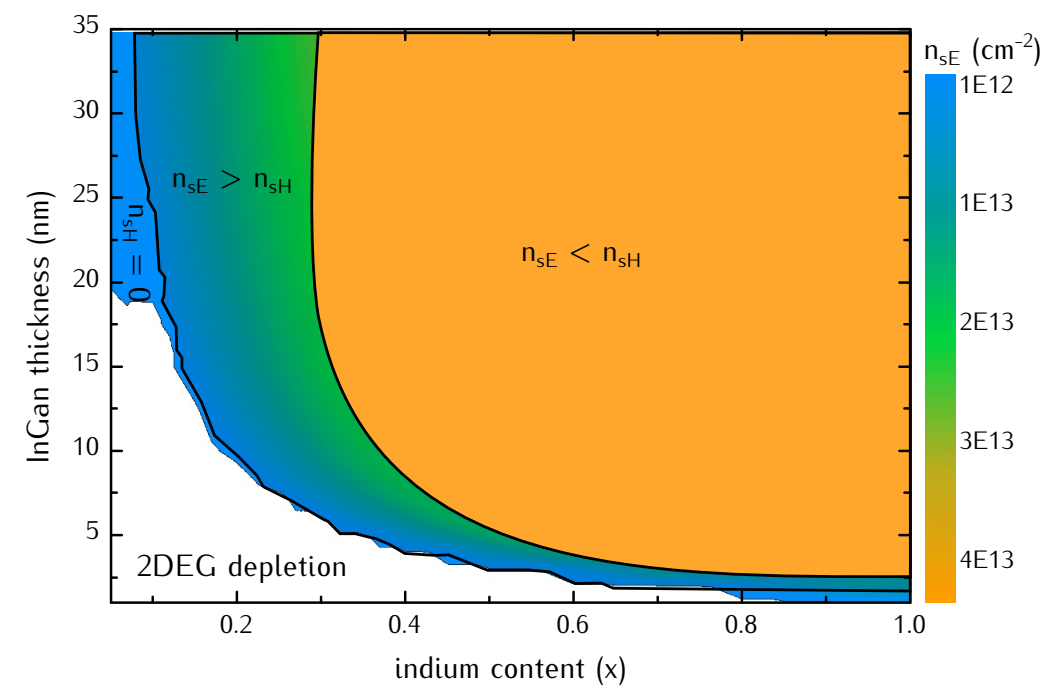

Figure 6.19: Electron concentration over indium content and InGaN thickness with $50 \mathrm{~nm}$ GaN cap layer. Four areas are indicated: 2DEG depletion, $n_{s E}>n_{s H}=0, n_{s E}>n_{s H}$ and $n_{s E}<n_{s H}$. Structures with only 2DEGs are limited to a very narrow range at low indium content. 


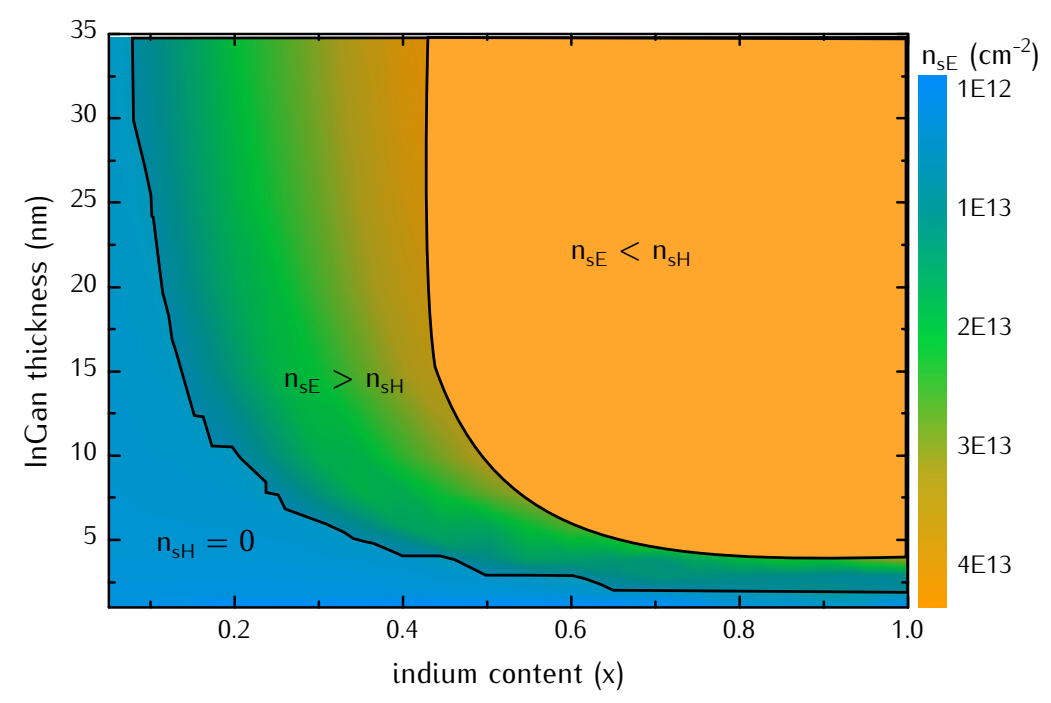

Figure 6.20: Electron concentration over indium content and InGaN thickness with $20 \mathrm{~nm} \mathrm{Al} \mathrm{l}_{0.2} \mathrm{Ga}_{0.8} \mathrm{~N}$ and $2 \mathrm{~nm} \mathrm{GaN}$ cap layer. Three areas are indicated: $n_{s E}>n_{S H}=0, n_{s E}>n_{S H}$ and $n_{s E}<n_{s H}$. Structures with only 2DEGs are achieved for various InGaN thicknesses.

for $n_{s E}>n_{s H}$, and structures with $50 \mathrm{~nm}$ cap layers are considered in the following. However, the number of holes increases stronger with the $\ln G a N$ thickness than $n_{S E}$, and $n_{S E}<n_{S H}$ is obtained for thick InGaN barriers with higher indium content even for thick cap layers.

A contour plot of $n_{s E}$ depending on InGaN thickness and In content for a $50 \mathrm{~nm}$ capped GaN/InGaN/GaN structure is shown in Figure 6.19. For thin layers with low indium content neither a 2DEG nor a 2DHG forms. At thicker barriers with high In content, mainly p-conductivity is expected. A range of structures with $n_{s E}>n_{S H}$ can be found. Structures with $n_{s E}>n_{s H}=0$ are, however, limited to low In concentrations.

By introducing an AlGaN layer as shown in Figure 6.16, the range of structures with $n_{s E}>n_{s H}=0$ can be extended as shown in Figure 6.20. The strained AlGaN layer induces an additional positive polarization charge at the InGaN/AlGaN interface while the negative charge at the GaN/InGaN interface remains unchanged (see Figure 6.16). As a result, $n_{S E}$ increases stronger by introducing the AlGaN layer compared to $n_{s} H$, but the critical thickness for 2DHG formation remains unchanged. Therefore, structures with only two dimensional electron accumulation are obtained at thinner InGaN barriers. The growth of GaN/InGaN/AlGaN/GaN structures with thin InGaN layers therefore reduces the contribution of a p-conductive channel.

\subsection{MAGNETOTRANSPORT RESULTS}

The effects of growth optimization on the structural morphology are reflected by the transport properties of grown samples. Figure 6.21 gives an overview 
of the mobility of herterostructures with In contents between $x_{/ n}=0.10$ and $x_{/ n}=0.25$ measured in the van der Pauw geometry at $10 \mathrm{~K}$. No 2DEG could be detected in structures grown at substrate temperatures below $T_{s}=550{ }^{\circ} \mathrm{C}$. Here, the mobility in GaN/InGaN/GaN structures could not be distinguished from bulk GaN.

Growth at higher substrate temperatures under In bilayer stabilized conditions resulted in an increase of the mobility up to $\mu=100 \mathrm{~cm}^{2} V^{-1} \mathrm{~s}^{-1}$. The use of excess Ga as described in Section 6.2 further increases the mobility to almost $\mu=300 \mathrm{~cm}^{2} V^{-1} \mathrm{~s}^{-1}$. The highest mobilities were obtained for structures with additional AlGaN layer. However, neither a variation of the sheet carrier density nor an additional AlGaN layer showed a reproducible impact on $\mu$.

Hall bar measurements showed higher mobilities compared to measurement in the vdP geometry as shown in the inset in Figure 6.21. This is expected, because the probed area is smaller and inhomogeneities have a smaller impact on the results. This method also revealed deviations of the electronic properties for different positions on the samples. Differences of a factor of two in $n_{s}$ and $\mu$ have been measured for different Hall bars on the same sample.

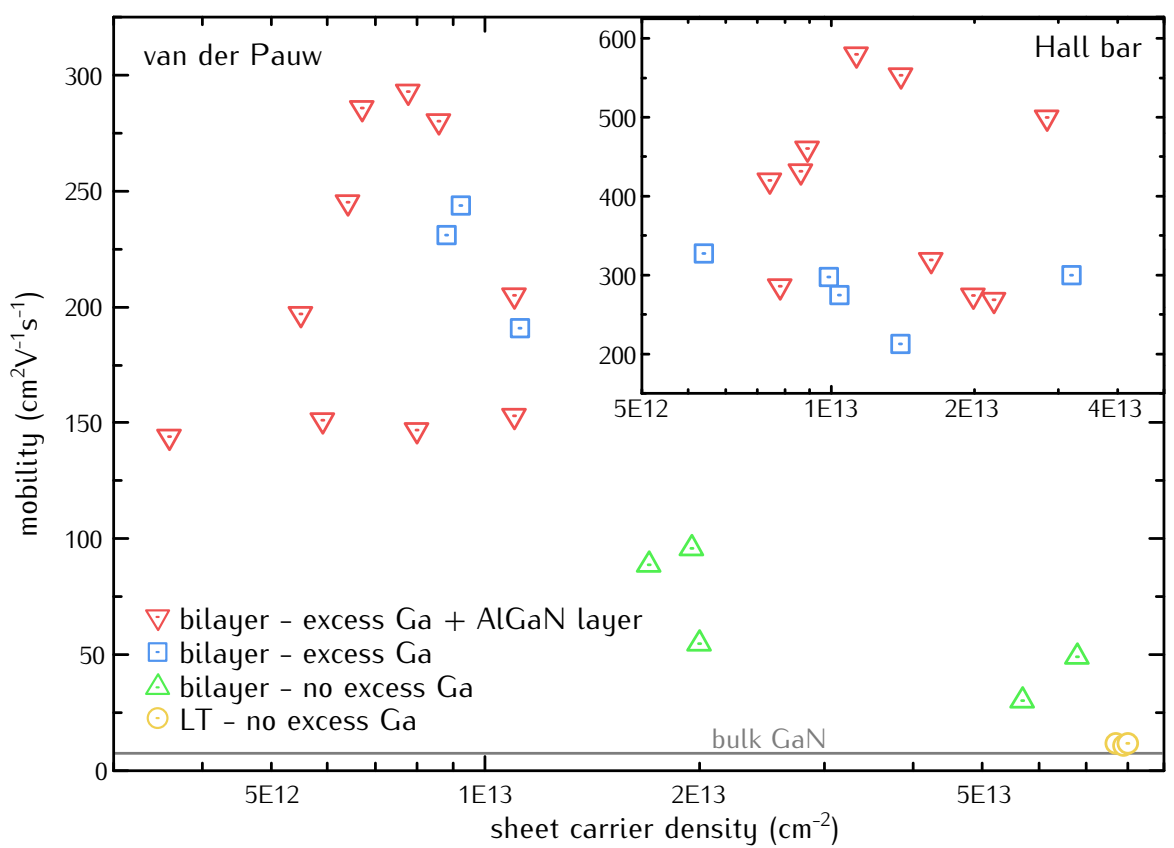

Figure 6.21: Mobility over $n_{s}$ for various GaN/InGaN(/AlGaN)/GaN structures measured in the vdP at $10 \mathrm{~K}$ and in the Hallbar geometry at $4 \mathrm{~K}$. It illustrates the impact of different growth optimizations steps: growth at low temperatures (LT) without the indium bilayer, indium bilayer stabilized growth with and without excess Ga and structures with additional AlGaN layers. The highest mobilities were achieved for structure with additional AlGaN layers grown under indium bilayer conditions with excess $\mathrm{Ga}$ before and after InGaN growth. 


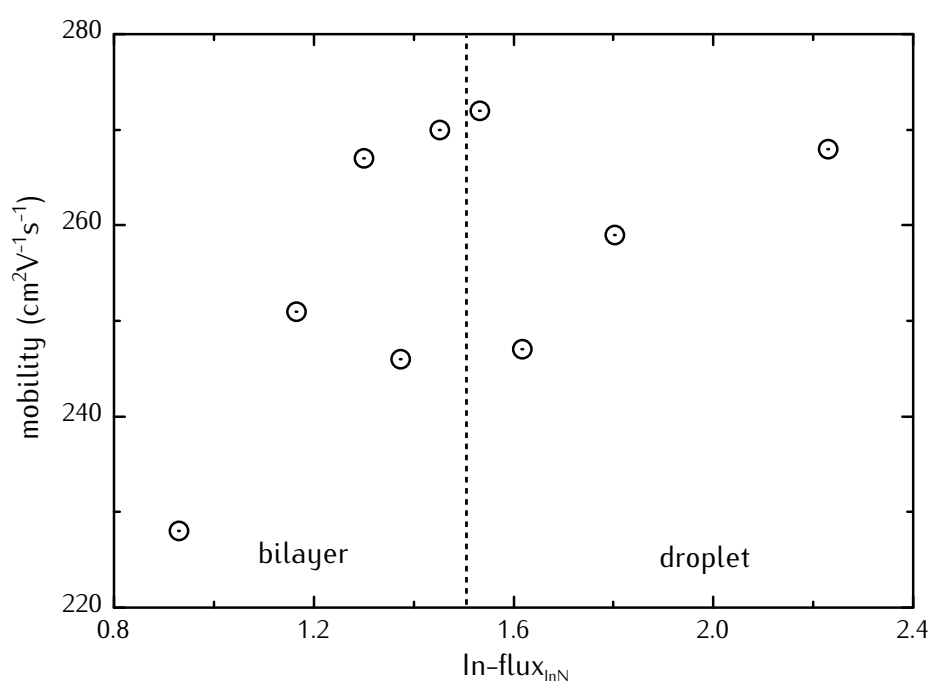

Figure 6.22: Mobility over indium flux measured in the vdP geometry at $10 \mathrm{~K}$ for $10 \mathrm{~nm} \ln _{0.15} \mathrm{Gaa}_{0.85} \mathrm{~N} / 15 \mathrm{~nm} \mathrm{Al}_{0.2} \mathrm{Ga}_{0.8} \mathrm{~N} / 2 \mathrm{~nm} \mathrm{GaN}$ structures. The Influx is given normalized to $\ln N$ stoichiomtry. No clear dependence of $\mu$ is observed for different indium supplies.

These inhomogeneities are likely the result of the applied growth conditions. At indium bilayer conditions, small fluctuations in substrate temperature or metal flux generate significant changes in the In concentration. This also explains the poor reproducibility of transport properties in grown samples. A low reproducibility was observed even though great care was taken to maintain comparable growth conditions between different samples. Series of structures were grown subsequently without other samples grown in between. The stoichiometric conditions were checked before longer growth series, and the incorporation rate was controlled by XRD measurements frequently. Apart from bilayer growth and excess Ga, no reproducible impact of other changes of growth conditions on the low-temperature mobility was observed.
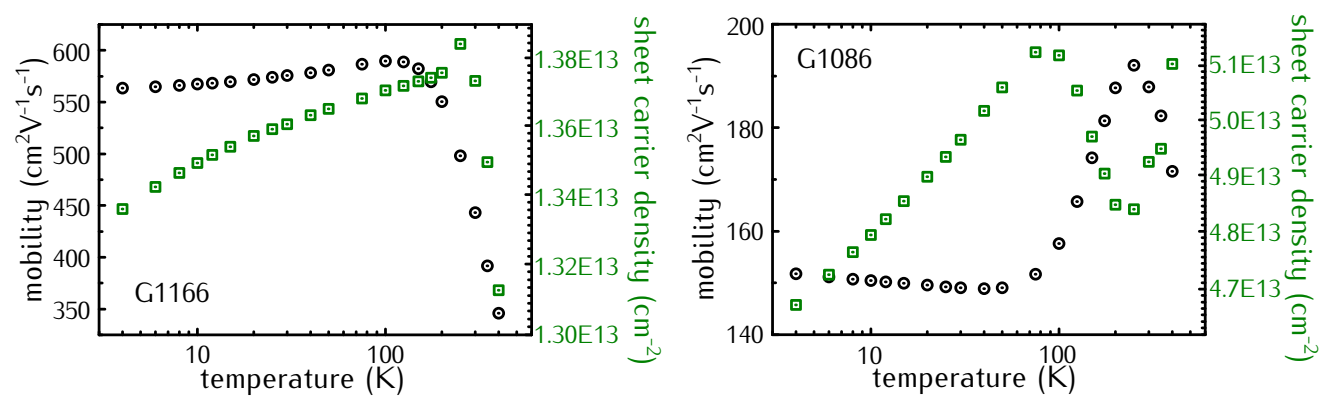

Figure 6.23: Mobility and $n_{s}$ over temperature for two G1166: $10 \mathrm{~nm} \ln _{0.15} \mathrm{Ga}_{0.85} \mathrm{~N} / 15 \mathrm{~nm} \mathrm{Al} 0.2 \mathrm{Ga}_{0.8} \mathrm{~N} / 2 \mathrm{nmGaN}$ and G1086: $25 \mathrm{In}_{0.17} \mathrm{Ga}_{0.83} \mathrm{~N} / 25 \mathrm{~nm} \mathrm{Al} \mathrm{.}_{0.2} \mathrm{Ga}_{0.8} \mathrm{~N} / 25 \mathrm{~nm} \mathrm{GaN}$. The dependencies indicate a 2DEG influence by a 2DHG, bulk GaN and electron-electron interaction. 
Among those were a variation of the indium content, growth at different substrate temperature within the bilayer regime and variations of amount of excess Ga. As an example, of how the low reproducibility influenced the measurements is shown in Figure 6.22. Here, the mobility was measured for samples with identical structure grown with varying In flux. It is apparent that the data is not meaningful.

Temperature dependent transport measurements for various heterostructures indicated two dimensional conductance. This is illustrated by $\mu(T)$ and $n_{s}(T)$ for two selected GaN/InGaN/AlGaN/GaN samples in Figure 6.23. For sample G1166, the mobility is almost constant for low temperatures and drops above $100 \mathrm{~K}$, which is expected for a two dimensional carrier gas. The observed $n_{s}(T)$ dependencies are not characteristic for 2DEGs or 2DHGs, which would imply a constant $n_{s}$ at low temperatures and an increase above $100 \mathrm{~K}$ due to an increasing contribution from bulk electrons. Both structures presented in Figure 6.23 show a small increase of $n_{s}(T)$ for low temperatures. This is not an actual change of the carrier density, but rather the result of electron-electron interaction [136]. This scattering process has a temperature dependent impact on the slope of the Hall voltage, which is used to calculate $n_{s}$.

A 2DHG was present in some structures. This was concluded from the reduction of $n_{s}$ above $100 \mathrm{~K}$, as observed for both samples shown in Figure 6.23. Transport measurements include different conductive channels in a heterostructure depending on their conductivity $\sigma=e n_{s} \mu$ (see Section 3.3).

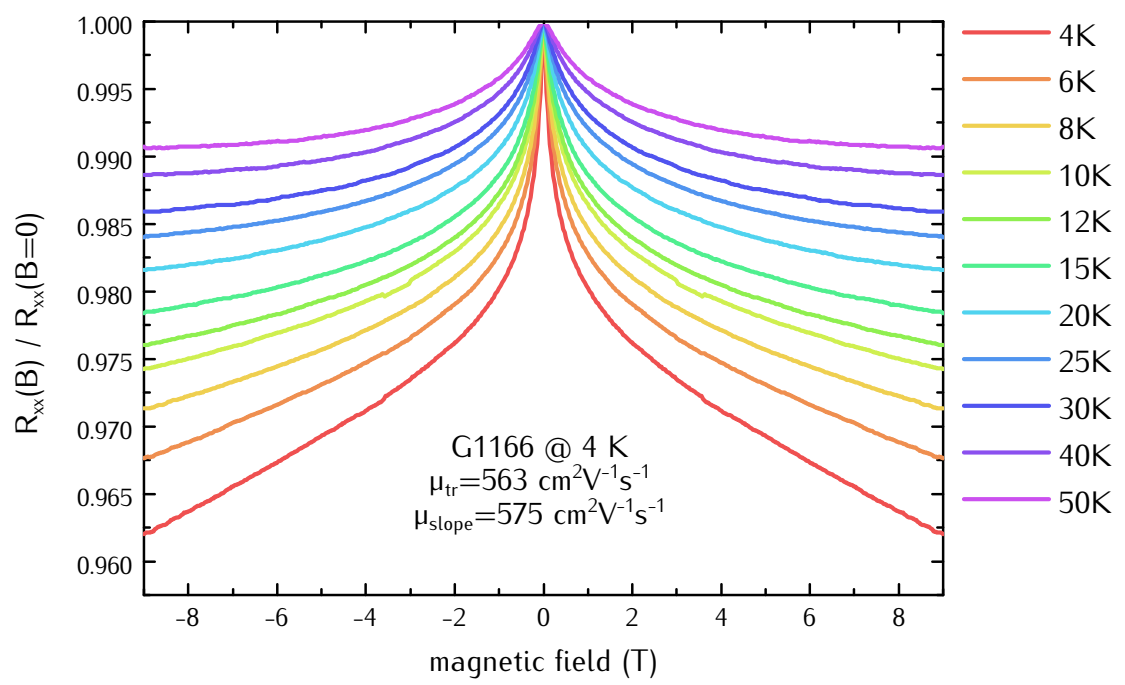

Figure 6.24: Normalized magnetoresistance of sample G1166 for various temperatures. Two contributions are observed, both depending on temperature. A peak around $B=0 T$ is the results of weak localization. The linear decrease of $R_{x x}(B)$ at higher fields originates from a potential of impenetrable discs. The mobility calculated from the slope is in good agreement with the mobility obtained from Hall measurements. 
The sheet carrier density calculated from the Hall voltage is thus a measure of the mean $n_{s}$, i.e. in the case of a 2DEG and a 2DHG $n_{s}=n_{s E}-n_{s} H$. The increase of $n_{s}(T)$ observed above $150 \mathrm{~K}$ in sample G1086, is the result of an increased conduction from the bulk GaN. Note that 2DHG and bulk contributions to $n_{s}$ have a strong impact on $\mu$. Therefore, only the mobility at low temperatures is a reliable measure of the mobility in a 2DEG in grown heterostructures.

In order to identify the dominating scattering mechanisms and to confirm the existence of a 2DEG at low temperatures, the magnetoresistance of selected samples was studied in detail. As an example, the normalized longitudinal resistance $R_{x x}(B) / R_{X X}(B=0 \mathrm{~T})$ for sample $\mathrm{G} 1166$ is given in Figure 6.24. It should be noted that the data was symmetrized by $R_{x x}( \pm B)=0.5\left[R_{x x}(B)+R_{x x}(-B)\right]$. This has to be applied for magnetic field depended four-probe measurements, which are always influenced by asymmetries [137, 138, 100]. Two strong temperature dependent features are observed: a peak around zero field and a linear dependence of $R_{x x}(B)$ on $B$ for $B>3 T$.

The peak arises from weak localization (see Section 3.4.2). Its shape depends on $l_{e} / l_{i}$ and $l_{e}$, and the elastic mean free path $l_{e}$ and the inelastic mean free $l_{i}$ can be calculated by fitting the data around $B=0$ T. For sample G1166, $l_{e}=31 \mathrm{~nm}$ and $l_{i}=94 \mathrm{~nm}$ at $4 \mathrm{~K}$ were obtained. The temperature dependence of the inelastic relaxation time is characteristic for the inelastic scattering process and dimension of conductance [111]. For inelastic scattering induced by electron-electron interaction, a $\frac{1}{\tau_{i}} \propto T^{p}$ dependence is expected, with $p=2$ for 3D and $p=1$ for 2D carrier systems. For two dimensional transport in a disordered system, a $\frac{1}{\tau_{i}} \propto T \ln \frac{1}{T}$ dependence should be observed.

The inelastic relaxation time could not be calculated from $l_{i}=\sqrt{D \tau_{i}}$, because the diffusion constant $D$ was not known. Therefore, the temperature dependence of $D \tau_{i}(T)$ was studied. For sample G1166, $D \tau_{i}(T)^{-1} \propto T$ and $D \tau_{i} T^{-1} \propto T \ln \left(T^{-1}\right)$ are compared in Figure 6.25. The latter describes the results best, which confirms two dimensional conductance. The disorder, for which this dependence is expected, is explained by the conductance within the InGaN barrier which is subject to alloy disorder and fluctuations of the indium content.

The linear contribution to the magnetoresistance results from scattering at a potential described by randomly distributed impenetrable discs with radius $a$ and a mean separation distance of $l_{a}$ (see Section 3.4.3). This description actually predicts three different $R_{x x}(B)$ trends, with transitions depending on $a / l_{a}$. The resistance should be proportional to $B^{2}$ for $B<0.05 a / l_{a} \mu^{-1}$, while at $B>2 a / l_{a} \mu^{-1}$ a $B^{-1 / 2}$ dependence is expected. At intermediate magnetic fields, the magnetoresistance should be linear with a slope, inversely proportional to the mobility. Even though, only a linear $R_{x x}(B)$ dependence is observed, it is reasonable to assume that the model applies at low temperatures as the mobility obtained from the slope compares well 


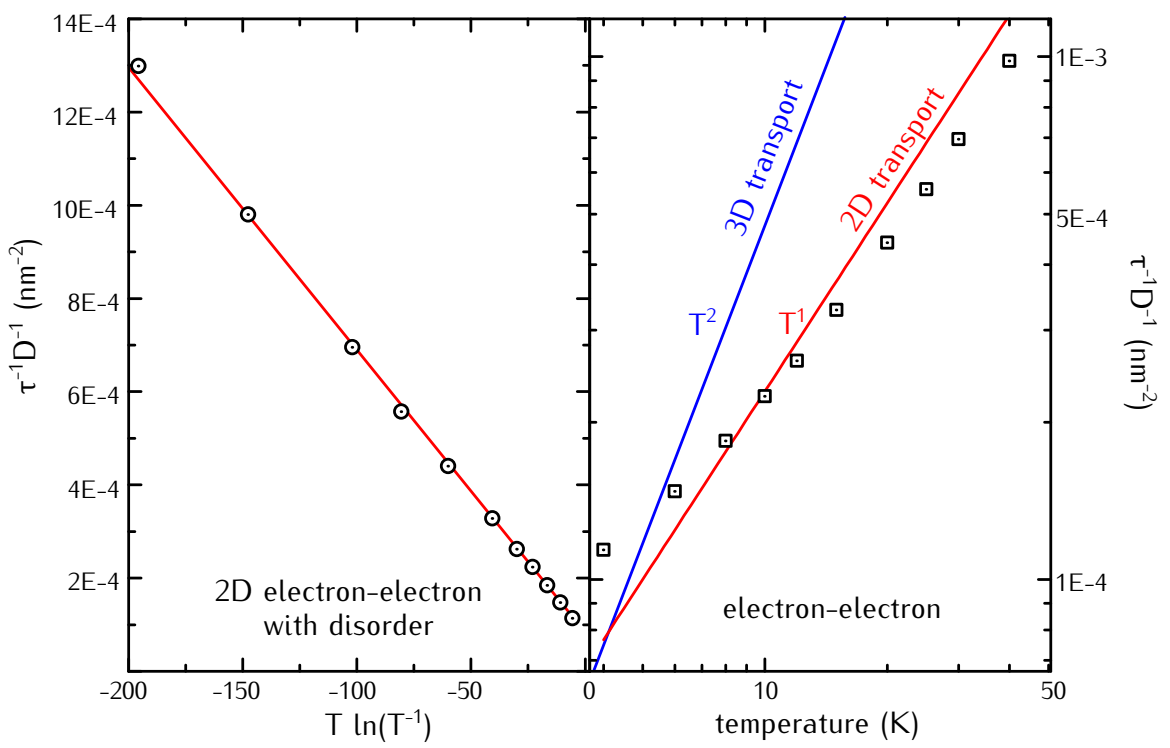

Figure 6.25: Comparison of different dependencies of inelastic scattering on temperature. $T^{2}$ indicates $3 \mathrm{D}$ and $T^{1}$ indicates $2 \mathrm{D}$ transport dominated by electron-electron scattering. The results are best described by a dependence of $D \tau_{i}$ on $T \ln \left(T^{-1}\right)$ which is expected for $2 \mathrm{D}$ transport in a discorded medium dominated by electron-electron scattering.

to the value obtained by Hall measurements $\left(\mu_{\text {slope }}=575 \mathrm{~cm}^{2} \mathrm{~V}^{-1} \mathrm{~s}^{-1}\right.$ to $\mu_{\text {hall }}=563 \mathrm{~cm}^{2} \mathrm{~V}^{-1} \mathrm{~s}^{-1}$ for $\mathrm{G} 1166$ at $4 \mathrm{~K}$ ). It is therefore presumed, that the $B^{-1 / 2}$ dependence was not observed because the transition occurs at $B>9 T$ and the $B^{2}$ dependence is overlaid by the weak localization peak at $B<3 \mathrm{~T}$. This allows to estimate the radius $a$ of the discs, if the mean free path $l_{e}$ obtained from the $\mathrm{WL}$ analysis is taken as $l_{a}$. A possible radii range for scatters of $7.5 \mathrm{~nm}<a<100 \mathrm{~nm}$ was found. It has to be noted, that $\mu_{\text {hall }}$ and $\mu_{\text {slope }}$ were only comparable at $4 \mathrm{~K}$, and the model does no apply at higher temperatures.

The dominating elastic scattering mechanism could not be identified directly from magnetoresistance measurements. However, results from transport measurements were compared to results obtained AFM and TEM and their impact on the band profile. Atomic force micrographs showed atomically flat terraces with a width of about $100 \mathrm{~nm}$. Transmission electron micrograph showed no additional dislocations incorporated during MBE growth. The mean separation between dislocations is therefore $500 \mathrm{~nm}$, corresponding the nominal dislocation density of the substrate of $4 \times 10^{8} \mathrm{~cm}^{-2}$. Both length scales are higher than the mean free path of $l_{e}=31 \mathrm{~nm}$, and interface roughness or dislocations are therefore not the source of the dominant elastic scattering process. The electron mobility at low temperatures should thus be limited by fluctuations in the In content, which have been observed by TEM. The impact of the possible scattering sources on the conduction band minimum (CBM) profile along the InGaN/GaN interface is illustrated in Fig- 


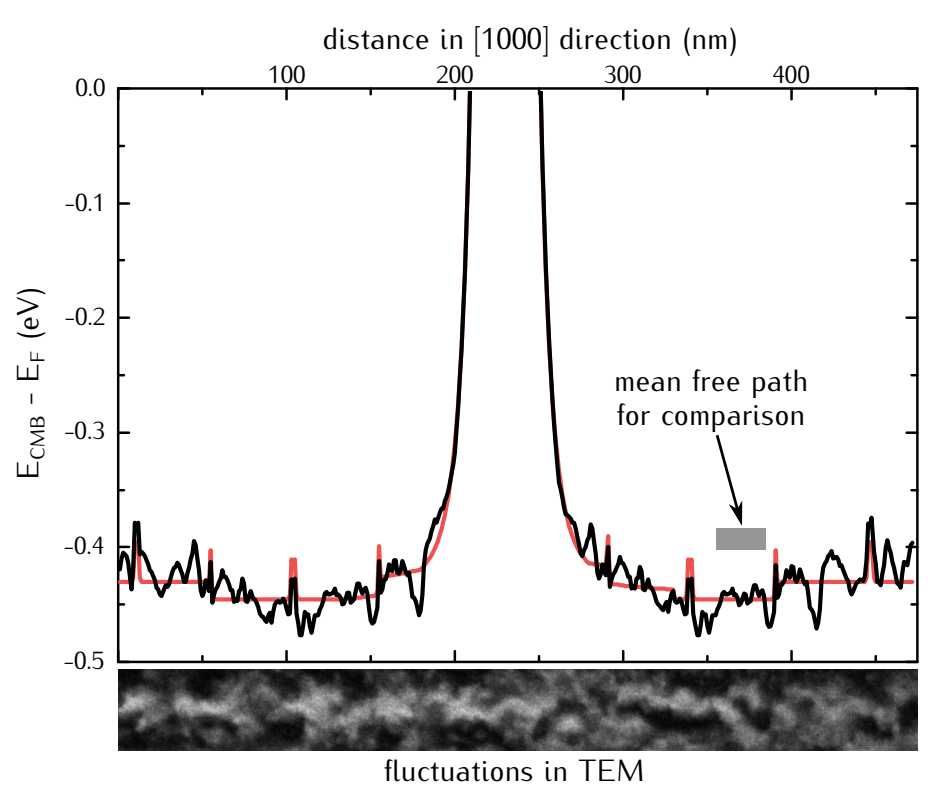

Figure 6.26: Illustration of the impact of atomic steps, a dislocation and indium fluctuations on CBM of a 2DEG in a GaN/InGaN/AlGaN/GaN heterostructure. The profile of indium fluctuations was estimated from the intensity of a transmission electron micrograph. The red line shows the impact of a dislocation and of atomic steps only. The comparison with the mean free path obtained from transport measurements suggest that indium fluctuations mainly influence the transport in InGaN based heterostructures at low temperatures.

ure 6.26. Atomic steps from surface roughness and dislocations were modeled by 2D Schrödinger-Poisson calculations as was described in Section 5.3. The profile of indium fluctuations was obtained from the intensity of TEM micrographs. The amplitude of the fluctuations of $\Delta x_{/ n}=0.04$ for a total indium content of $x_{/ n}=0.12$ was taken from literature [65]. It is observed that the height of the potential change is comparable for steps and fluctuations. However, it is evident that the variations in CBM induced by the indium fluctuations dominate the potential landscape due to their profile. $A$ comparison with the mean free path shows suggests that indium fluctuations are likely to be the source of the dominant elastic scattering that limits the electron mobility in 2DEGs at low temperatures. 



\section{SUMMARY OF OPTIMIZED GROWTH CONDITIONS}

At this point, the optimization steps and growth conditions that produced the best results are summarized.

Substrates were cleaned by acetone and 2-propanol in an ultrasonic bath with low power. They were then heated at $200{ }^{\circ} \mathrm{C}$ for $10 \mathrm{~min}$ and at $600{ }^{\circ} \mathrm{C}$ for $10 \mathrm{~min}$ under vacuum. Before growth, the surface was treated with activated nitrogen for $15 \mathrm{~min}$ at $700{ }^{\circ} \mathrm{C}$. No additional dislocations formed at the substrate/MBE interface if this treatment was applied.

The nitrogen plasma conditions were $300 \mathrm{~W}$ at $0.5 \mathrm{sccm}$ for all structures. The resulting growth rate at stoichiometry was $3 \mathrm{~nm} / \mathrm{min}$. The ratio between molecular to atomic nitrogen was 1.5. These conditions guaranteed stable plasma operation at relatively low powers to prevent damage from activated nitrogen.

For GaN growth, $T_{s}=700{ }^{\circ} \mathrm{C}$ was chosen, because it is below the expected decomposition temperature of $720^{\circ} \mathrm{C}$ and results in the highest Ga diffusion length. For AlGaN growth, $T_{s}$ was not changed compared to GaN growth, to reduce the growth interruption time.

The best results for GaN growth in terms of Ga-flux were achieved at the bilayer/droplet transition. This point was identified by studies of the RHEED intensity during Ga desorption. The flux was not changed for AlGaN growth. Smooth surfaces and sharp interfaces were obtained for these conditions. During GaN growth, the excess Ga was desorped every 7.5 min until only the Ga bilayer was left on the surface. The modulated growth reduced droplet formation, increased the reproducibility and the homogeneity and allowed for better control of the growth parameters.

InGaN was grown between $T_{s}=590^{\circ} \mathrm{C}$ and $T_{s}=650{ }^{\circ} \mathrm{C}$. This allowed for sufficient indium desorption to prevent droplet formation and enable growth under indium bilayer stabilized conditions, which gave the best results. In the applied temperature range, $x_{/ n}$ highly depends on $1-\mathrm{Ga} / \mathrm{N}, T_{S}$ and the indium flux. This resulted in a poor reproducibility and homogeneity of the indium content. The dependence of $x_{\ln }$ on $T_{s}$ limits the indium content in InGaN layers grown under indium bilayer stabilized conditions to $x_{\ln }=0.3$. A degradation of the surface was found for indium desorption, which coincides with the observation of a $\sqrt{3} \times \sqrt{3} \mathrm{R} 30^{\circ}$ reconstruction. The effect was counteracted by covering the surface with a protective Ga adlayer throughout growth. This resulted in smooth morphologies similar to GaN and AlGaN. 

Part III

CONCLUSION 



\section{SUMMARY, APPRECIATION AND OUTLOOK}

In this work, the MBE growth of GaN (0001) based 2DEG heterostructures with InGaN and AlGaN quantum wells was optimized with respect to the low temperature electron mobility. For InGaN based heterostructures mobilities as high as $\mu=560 \mathrm{~cm}^{2} \mathrm{~V}^{-1} \mathrm{~s}^{-1}$ at $4 \mathrm{~K}$ were obtained which is the highest reported value for $\mathrm{MBE}$ grown material today. The mobilities were, however, to low to investigate, e.g. the effective mass or spin transport effects. State of the art results were achieved for GaN/AlGaN structures with a reproducible mobility around $\mu=20000 \mathrm{~cm}^{2} \mathrm{~V}^{-1} \mathrm{~s}^{-1}$ at $2 \mathrm{~K}$. For each material system, the optimization process was studied in detail using in-situ RHEED. A number of key aspects that significantly affect the electron transport were identified. The dominating scattering processes in InGaN and AlGaN based two dimensional electron gases were determined by magnetoresistance measurements together with structural characterization.

Three optimization steps significantly improved the GaN (0001) quality: nitridation of the substrate, modulated growth with desorption steps and a Ga/N ratio at the bilayer-droplet transition. No additional dislocation formed at the substrate/MBE interface after treatment with activated nitrogen at growth temperature. Modulated growth monitored by in-situ RHEED improved the growth in three aspects. First, the regular desorption compensated the formation of droplets from accumulating Ga under Ga-rich conditions. Secondly, the homogeneity increased because the droplet formation on colder parts of the sample decreased. Thirdly, the reproducibility and overall sample quality increased because the desorption, which is characteristic for different metal-rich growth conditions, was monitored during growth. This allowed to compensate deviations in material fluxes and substrate temperature during growth and over longer growth series. The electron mobility of GaN/AlGaN structures showed a strong dependence on the $\mathrm{Ga} / \mathrm{N}$ ratio with a maximum mobility at the bilayer-droplet transition. The growth of thin AlGaN layers with low Al content was carried out under Ga bilayer stabilized growth conditions. The substrate temperature and the Ga-flux were not changed compared to GaN growth to reduce the growth interruption time.

In order to achieve high electron mobilities, the heterostructures were designed according to results of self consistent Schrödinger-Poisson calculations. Maximum mobilities have been achieved around a sheet carrier 
density $n_{s}=2 \times 10^{12} \mathrm{~cm}^{-2}$, which is in close agreement with the literature. It was presented that such low densities can be achieved for a broad range of AlGaN thicknesses for low Al contents.

The investigation of the low-temperature magnetoresistance provided insight into the dominant scattering mechanisms and the potential landscape of the two dimensional channel as visualized in Figure 7.1. Scattering times were calculated from Shubnikov-de Haas oscillations. A comparison of the transport relaxation time to the quantum relaxation time identified scattering at dislocations as the dominating scattering process. The mean free path verified that the number of dislocations is determines by the substrate.

As an application, AlGaN based $\mathrm{pH}$ sensors were studied. Such devices operate at room temperature where scattering at phonons dominates the transport. Structures for high sensitivity thus have to meet other requirements than structures optimized for high electron mobility at low temperatures. Therefore, heterostructures for high sensitivity were designed according to

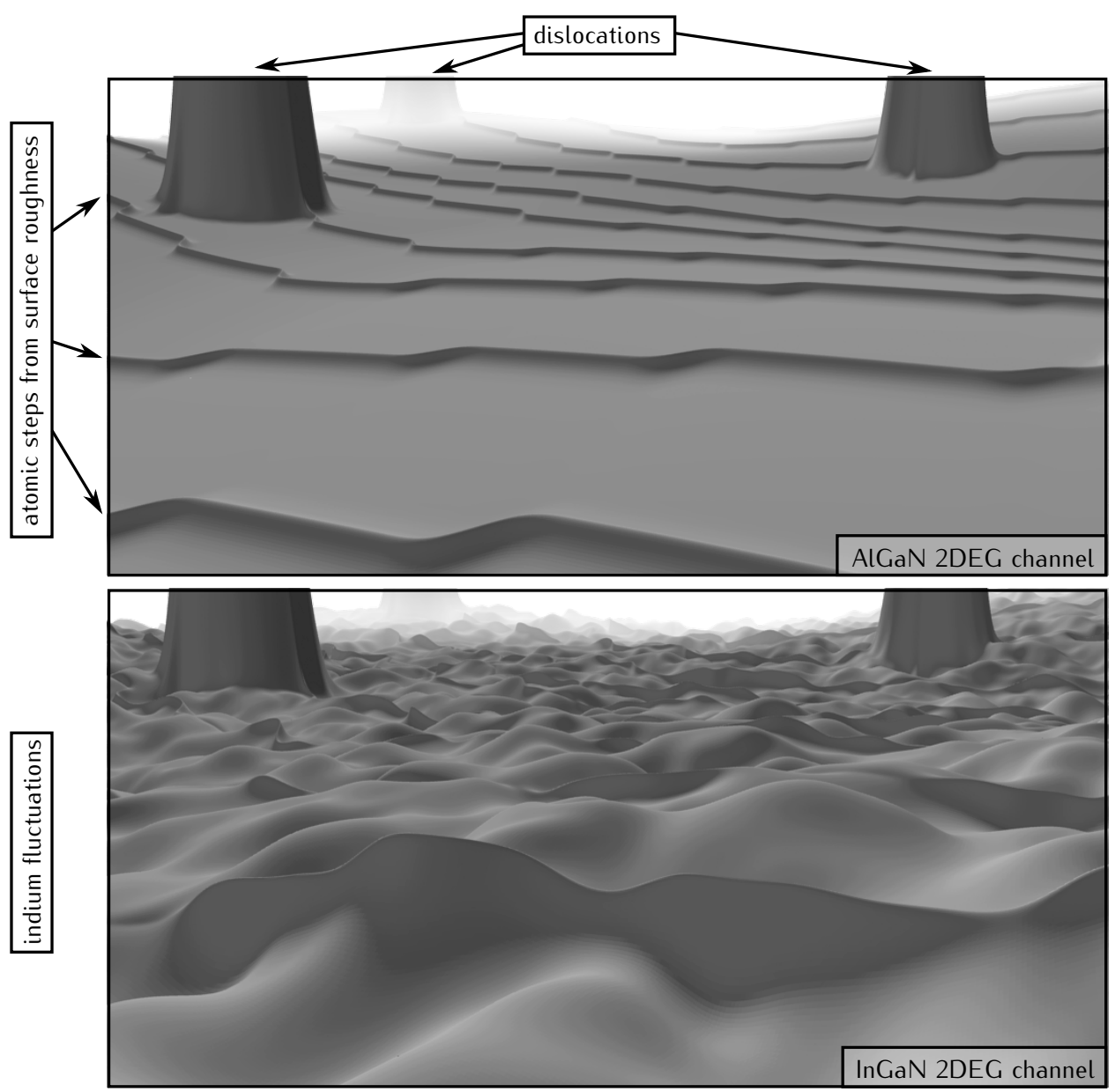

Figure 7.1: Visualization of the quantum well potential of a 2DEG in AIGaN and InGaN along the GaN/AlGaN and InGaN/GaN interface for a $1 \times 1 \mu \mathrm{m}$ area. Dislocations limit the mobility for GaN/AlGaN quantum wells, while indium fluctuations result in low mobilities for InGaN wells. 
the results of self consistent Schrödinger-Poisson calculations. Structures with channels close to the surface showed high response to $\mathrm{pH}$ solutions and are currently functionalized for drug screening at the university of Western Australia in Perth.

Two optimization steps for InGaN significantly improved the mobility in GaN/InGaN(/AlGaN)/GaN heterostructures: growth under In bilayer stabilized conditions and the suppression of surface degradation through excess Ga. Sufficient indium desorption is required for In bilayer stabilized growth and the substrate temperature was thus increased above the decomposition temperature of $\ln N$. In this temperature range, the In incorporation depends on $1-\mathrm{Ga} / \mathrm{N}$, the substrate temperature and the indium flux. The maximum incorporable amount of indium was estimated by a novel RHEED method. Because of the dependence on various parameters, a reproduction of the indium content was challenging.

An investigation of the morphology of InGaN surfaces after indium desorption revealed a post growth degradation. This coincided with the observation of a $\sqrt{3} \times \sqrt{3} \mathrm{R} 30^{\circ}$ by RHEED. The effect was compensated by applying excess Ga before and after InGaN growth. This resulted in atomically flat morphologies and low dislocation densities, both being comparable to AlGaN based heterostructures.

The contribution of holes to 2D transport was minimized according to the results of Schrödinger-Poisson calculations. It was found that heterostructures with InGaN wells in which only a 2DEG forms, are limited to low In content. By introducing an additional AlGaN layers, this range can be extended. However, no reproducible improvement of the measured transport data at low temperatures was achieved.

Despite the structural improvements, the low-temperature mobility remained low in InGaN based heterostructures compared to AlGaN 2DEGs. Analysis of the magnetoresistance at low temperatures showed a truly two dimensional conductance with a mean free path of $30 \mathrm{~nm}$. Calculations of the band profile along the InGaN/GaN interface that took the results from TEM measurements into account, suggested indium fluctuations as the source of the dominating scattering processes for the applied growth conditions. This is visualized in Figure 7.1. It is apparent that the fluctuations prevent a further increase of the mobility by smoother interfaces or a lower dislocation density.

\subsection{APPRECIATION AND OUTLOOK}

This work demonstrated how thorough optimization can increase the quality of III-nitrides. Several obstacles for the MBE growth of nitrides were presented, most of which were overcome. However, the limits that were reached go beyond MBE growth optimization. In the end, bigger challenges remain which the material system has been facing since the beginning. In the case 
of InGaN, they are fluctuations of the indium content. For AlGaN, it is the lack of substrates with low dislocation densities.

A further improvement of the low temperature electron mobility of InGaN based heterostructures by MBE is questionable. One approach to reduce the fluctuations could be growth with a decreased indium diffusion. This could be achieved by growth at lower temperatures. However, this results in rougher surfaces. A compromise between fluctuations and roughness should result in higher mobilities. Another approach could be an increased growth rate which would reduce the surface diffusion of indium.

The electron mobility in AIGaN based structures at this stage of optimization can only be increased by better substrates. The structures presented in this work were grown on $3 \mu \mathrm{m}$ MOVPE templates with dislocation densities of $4 \times 10^{8} \mathrm{~cm}^{-2}$. Thicker templates grown by MOVPE and HVPE with densities as low as $10^{7} \mathrm{~cm}^{-2}$ and recently available 2-inch bulk GaN wafers with densities $<10^{5} \mathrm{~cm}^{-2}$ promise better results.

A point of principle is, what profit an increased mobility would have. This is different for InGaN and AlGaN. A further increase of the mobility in GaN/AlGaN structures grown by MBE requires better substrates, and therefore comes with higher costs. Instead of a further MBE growth optimization of GaN and AlGaN, future work in this field should therefore focus on new applications. As an example, non toxic sensors were studied as part of this work.

For device applications of heterostructures with InGaN wells, it is of lesser interest to increase the mobility. The obvious application for high electron mobility structures are transistors. In principle, InGaN structures promise a higher mobility than AlGaN devices, because the effective mass of electrons is lower in InN than in GaN or AIN. However, the 2DEG in GaN/InGaN/GaN forms within the InGaN layer, and is therefore subjected to alloy scattering. The effective mobility in 2DEGs would thus be of the same order in heterostructures with AlGaN and InGaN quantum wells, if the indium fluctuations are fully suppressed. In addition, indium is an extremely rare and expensive metal and will not be used for something other materials can do just as fine. For optoelectronic devices, a suppression of the indium fluctuations is not desirable. Areas with higher indium content are the source of efficient radiative recombination. Therefore, there is no benefit for applications from an increased mobility by a reduction of the fluctuations.

From a research perspective, higher mobilities in InGaN 2DEGs are desirable. If the mobility could be increased by only one order of magnitude compared to the results obtained in this work, basic material properties, such as the effective mass or spin-orbit interaction, can be accessed by electrical measurements. This could be compared to results from other techniques in order to improve the understanding of this material system, and motivates future work on the MBE growth optimization of InGaN. 
LAB HANDBOOK 



\section{A}

\section{LETS GET GROWING}

\section{A.1 WELCOME TO THE SAMPLE WASH}

If you want to grow high quality samples, and since you are one the very very very few people I assume to read this, I bet you want do, you need a clean substrate. The first step towards a clean substrate is a clean sample holder. In the Veeco GEN II MBE used in this work, the samples are mounted onto molybdenum holders. Over time, these holder get covered with things you deposited there, and other things you do not want there. Therefore, the holder should be cleaned about every 6 month. In order to remove heavy contaminations, the holder has to be ground. Afterwards, chemical cleaning removes residuals from the grinding process. You can remove light contaminations just chemically.

The chemical cleaning always has to be done by $\mathrm{HCL}$ and $\mathrm{KOH}$, of course after one another. $\mathrm{HCL}$ removes metals, and $\mathrm{KOH}$ removes oxides and nitrides. Molybdenum is hardly etched by both, and long treatments (about $30 \mathrm{~min}$ and more) in non diluted $\mathrm{HCL}$ and high molar $\mathrm{KOH}$ solutions can be applied. If possible, do this in an ultra sonic bath to support the process, but keep the acid and base covered to prevent evaporation. After the chemical cleaning the holder parts have to be rinsed in deionized water. Before mounting a sample, the empty holder has to baked at $200{ }^{\circ} \mathrm{C}$ for some hours in the intro chamber.

The cleaning process WILL change the effective temperature of the substrate. From many growth cycles the holder was covered by thick layers of metals and nitrides. When the layers are removed, the heat emission from the holder changes drastically. For the same substrate, I observed temperature differences as high $50^{\circ} \mathrm{C}$ in the reading temperature indicated by the temperature controller. The substrate temperature should be changed accordingly. Do so, by checking the desorption of a metal of your choice at a reference temperature you checked before cleaning the holder. The same holds when growing on a different sample holder. EVERY holder has a different heat emission either by design or by growth history, and believe me, it is not small.

For all the samples presented in this work, I used GaN templates from LUMILOG with a dislocation density of $4 \times 10^{8} \mathrm{~cm}^{-2}$. These substrates 
showed good results. Before placing a big order of substrates from other companies, the substrate quality should be checked on a test substrate. Grow one AIGaN 2DEG on LUMILOG and one on the new substrate AS THE NEXT SAMPLE. Do not let anybody else come between you and your work. By the way, the same is also true for delicate growth series. Successive growth will limit deviations of parameters you can not control or even think of. Rate the new substrate according to the low temperature mobility. If in the near future bulk GaN becomes affordable, BUY IT.

The substrates usually come epi-ready. Unfortunately, they have to be diced before growth which results in a slight contamination. A cleaning with solvents is enough to prepare them for growth. In this work, the following steps were applied and yes, they were applied twice, but in different beakers:

1. 2 min aceton with ultrasonic power 2

2. 2 min aceton with ultrasonic power 2

3. 2 min 2-propanol with ultrasonic power 2

4. 2 min 2-propanol with ultrasonic power 2

5. blow dry with nitrogen QUICK and THOROUGH

During this process the sample must never dry by itself as this WILL result in stains. For the same reason, the nitrogen pressure used to blow the sample dry has to be as high as it is, so grab your sample tight. Otherwise it will join many of my substrates in the gutter of the flowbox. Other solvent cleaning procedures are possible. However, do not assume that a procedure works without checking it. Control the surface by AFM and optical microscopy. Do so after bake out, because this will represent the surface you grow on.

Now you can mount the clean substrate onto the holder, but do not take it easily. The sample always has to be mounted in the same way. A tilt or a shift results in a changed temperature of up to $20^{\circ} \mathrm{C}$. After mounting the sample, heat it for $10 \mathrm{~min}$ at $200{ }^{\circ} \mathrm{C}$ in the load lock and for $10 \mathrm{~min}$ at $600{ }^{\circ} \mathrm{C}$ in the buffer chamber. Long periods of heating produce a carbon covered surface, so short times at higher temperatures are better.

When the sample is in the growth position, heat it up to the growth temperature. After the nitrogen plasma is on, open the nitrogen shutter for $15 \mathrm{~min}$. This will remove containments from the surface.

\section{A.2 WHERE STARS ARE BORN: THE GROWTH CHAMBER}

This is where the magic happens. When working on the machine, always remember: If you break it, many other people will loose it. As you probably had a good instruction by one of your fellow coworkers, I only state very few points here, you should bear in mind: 
- heat samples with a maximum ramp of $25 \mathrm{~K} / \mathrm{min}$.

- heat effusion cells with a maximum ramp of $15 \mathrm{~K} / \mathrm{min}$ (except Si: $40 \mathrm{~K} / \mathrm{min})$.

- do not flash GaN. If you heat it to high, it decomposes.

- regenerate the cryogenic pump at least 4 times a year (better every weekend).

- never let the liquid metal effusion cells drop below or rise above their melting point uncontrolled, as the crucible might break. For Ga this means: heat is slightly if opening the growth chamber.

I wasted a lot of time, by starting the nitrogen plasma wrong. To spare you this, here is the easiest way I found for my conditions:

1. CLOSE the ion pump valve.

2. open the molecular nitrogen supply.

3. set the flux to $2.5 \mathrm{sccm}$ or higher, until the chamber pressure exceeds $1.5 \times 10^{-5}$ mbar.

4. AFTERWARDS, start the high frequency generator at $300 \mathrm{~W}$ and reduce the reflected power to zero.

5. set the flux to $0.5 \mathrm{sccm}$ and wait, keeping an eye on the reflected power.

6. the pressure will decrease, because the flux was reduced. At some point there will be an instant drop (around $8 \times 10^{-6} \mathrm{mbar}$ ) and the reflected power jumps up. You are now in the high brightness mode.

7. reduce the reflected power to zero and check for the next $10 \mathrm{~min}$ if it stays stable.

\section{A.3 GAN THE GREAT}

In the following, I describe the optimization that resulted in my best samples. This is only a guideline, but it is a good point to start from. A sample recipe is given in Table A.1. The substrate temperature stated in the table is the temperature indicated by the temperature controller, it is not the real temperature I stated during the main part of my thesis. If you are confused see Appendix C.

After the 15 min of nitridation, a wetting layer of gallium is deposited to start the growth under Ga-rich conditions. Then a loop of 7.5 min growth and about $20 \mathrm{~s}$ desorption is started. During each loop, $25 \mathrm{~nm}$ of GaN grow 


\begin{tabular}{llllll}
\hline Material & Step & Time $[\mathrm{min}]$ & $T_{S}\left[{ }^{\circ} \mathrm{C}\right]$ & Ga & $\mathrm{N}$ \\
\hline \hline \multirow{2}{*}{ GaN } & Nitridation & 15 & $\approx 800$ & $\bigcirc$ & \\
\cline { 2 - 6 } & Ga wetting layer & 0.5 & $\approx 800$ & $\bigcirc$ & \\
\cline { 2 - 5 } & GaN & 7.5 & $\approx 800$ & $\bigcirc$ \\
\cline { 2 - 5 } & Desorption & 0.3 & $\approx 800$ & $\bigcirc$ \\
\hline
\end{tabular}

Table A.1: Recipe for GaN growth.

for a nitrogen flux of $0.5 \mathrm{sccm}$ with a power of 300 Watts.

Chose a Ga-flux 1.4 times higher than the GaN stoichiometry (roughly $p_{B E P}=2 \times 10^{-7}$ mbar at my time). You should choose the substrate temperature according to the desorption observed by RHEED. After closing the shutters for desorption, the RHEED intensity should rise within the next $20 \mathrm{~s}$. If it takes longer the substrate temperature needs to be increased. If the desorption start right away the substrate temperature needs to be lowered. For this method the first desorption step is usually misleading because of the wetting layer deposited prior to growth. If nothing happens after 1 min start nitridating until the RHEED intensity increases and immediately continue the growth. Start the adjustment of the substrate temperature with the second desorption loop.

It is always better to have an idea about the temperature range prior to growth. To evaluate the temperature roughly, deposit gallium on the template for $30 \mathrm{~s}$. If the RHEED intensity did not drop to a constant value during this time, the substrate temperature is way to hot. Decrease the temperature by $25^{\circ} \mathrm{C}$ and try again. If the RHEED intensity dropped to a constant value, monitor the desorption. If it takes more than 1 min till the intensity changes, the substrate is way to cold. Increase the temperature by $25^{\circ} \mathrm{C}$ and try again. If the desorption starts within 1 min start the growth. During the GaN loops the required adjustments should be in the range of only $\pm 10^{\circ} \mathrm{C}$. Try different RHEED directions or positions on the sample before making harsh changes, as no detectable desorption can also be based on a non ideal RHEED image.

The temperature adjustments during growth should only change the produced quality slightly, if the temperature was not to far off. For slightly non ideal condition, the surface roughness only changes a little bit. Because the roughness is only important at the interface towards another material, be sure you have everything under control by then. If the substrate temperature leads to no satisfying desorption, just keep growing and adjusting until it does and then continue with your heterostructure. Note, the samples 
presented in the main part were grown under constant conditions. The steps described here just represent a way to get started.

There is always the possibility, that you grew to metal rich, i.e. in the droplet regime. You can check this right after the sample comes out of the machine. Just wipe the tip of plastic tweezers over the grown sample. If this leaves a trace, there are metal droplets on the surface and the substrate temperature was to low. If you want to characterize a droplet covered sample, you can remove the droplets with $\mathrm{HCL}$ if you like. This will only remove metals and not etch nitrides.

\section{A.4 ALGAN THE ALMIGHTY}

AlGaN is easy to grow, if the layer is thin $(<30 \mathrm{~nm})$ and the Al content is small $\left(a_{A l}<0.25\right)$. Just open the Al shutter together with Ga and $\mathrm{N}$ after a regular GaN desorption step. For thicker layers and higher Al contents, the Ga-flux should be reduced according to desorption just as for GaN growth. $\mathrm{Al}$ is always preferably incorporated over Ga for c-plane AlGaN growth at typical GaN growth temperatures. An easy example: There are $100 \mathrm{~N}$ atoms, $100 \mathrm{Ga}$ atoms and $50 \mathrm{Al}$ atoms. The results will be $\mathrm{Al}_{0.5} \mathrm{Ga}_{0.5} \mathrm{~N}$ because $\mathrm{Al}$ gets served first. All aluminium atoms bind with the available nitrogen and gallium binds with the leftovers. This is why the Al content is given by the $\mathrm{Al} / \mathrm{N}$ ratio. If you know the stoichiometry of $\mathrm{AlN}$, the $\mathrm{Al}$ content is equal to the fraction of the stoichiometric Al-flux.

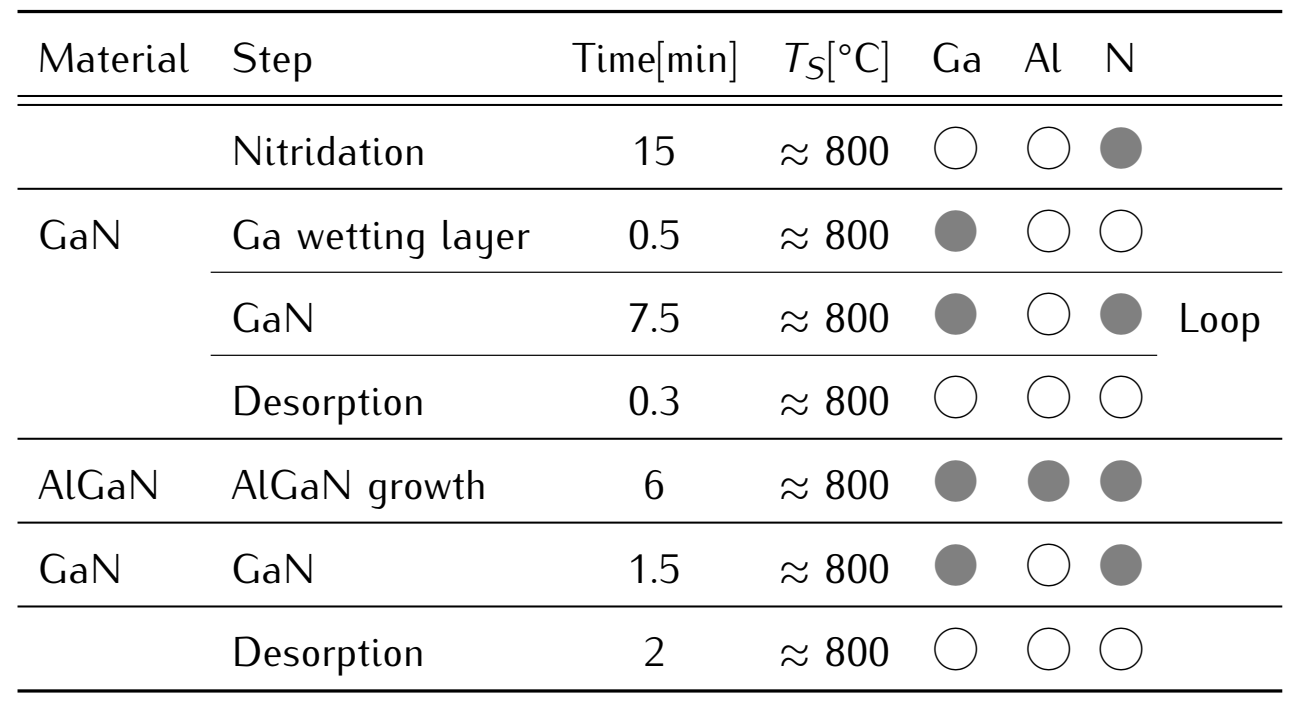

Table A.2: GaN/AlGaN/GaN recipe 
InGaN is way more feisty than GaN or AlGaN. There is still a lot to do, but this is as far as I got. One more thing before we start: InGaN with low indium content is yellow. Because it absorbs in the blue, all sample you have successfully incorporated indium into, will appear yellow to the naked eye. If you compare it to GaN templates, you can see the difference in color for InGaN layers thicker than $10 \mathrm{~nm}$. This is extremely useful if you grow at high temperature conditions, as you can check straight away if indium was incorporated or not. You should always double check with XRD, but it is still useful.

The hardest thing to control is the indium content, as it depends on the substrate temperature, the In-flux and the Ga-flux. You should therefore perform a RHEED study on a different sample before growing a series of samples. The following describes the growth with $10 \%$ In content, as an example.

First, find the GaN stoichiometry. Afterwards, chose a Ga-flux which corresponds to $90 \%$ of the stoichiometric flux for the following InGaN growth. Then chose an In-flux of about $p_{B E P}=3 \times 10^{-7}$ mbar. Do a RHEED study of the desorption with this flux depending on the substrate temperature. Start around $50{ }^{\circ} \mathrm{C}$ below the GaN growth temperature. Grow InGaN for $2 \mathrm{~min}$ and monitor the desorption. Reduce the substrate temperature until the growth is carried out under droplet conditions. At these conditions grow InGaN for $10 \mathrm{~min}$. If the sample grown appears yellow to the naked eye, the conditions were right and the actual sample can be grown. If it is not yellow, either the substrate temperature was to high or the $\mathrm{Ga} / \mathrm{N}$ ratio was higher than one. Try again.

During the growth of InGaN based heterostructures a protective gallium layer has to be present on the surface at all times. To do so, open the Ga shutter for about $1 \mathrm{~min}$ after the last Ga desorption step of the modulated GaN growth. Start to cool down the substrate during this deposition to the InGaN growth temperature obtained from the RHEED study. During the cool down Ga must not desorp, which has to be checked with RHEED and counteracted by the deposition of more Ga if necessary. While the sample cools down, the Ga-flux has to be reduced to $\mathrm{Ga} / \mathrm{N}=0.9$.

If at the InGaN growth temperature, deposit In for $30 \mathrm{~s}$. Now you have to get rid of the Ga adlayer, as it keeps indium from being incorporated. I tried various approaches here. One was, to let gallium desorp under excess indium. Another was to start growth by opening In $+\mathrm{Ga}+\mathrm{N}$. In the following I focus on a third approach, which is the easiest, but might not be the best. It implies that $\ln N$ does not grow at the applied substrate temperature. If at some point, the fluctuations should not be the dominant scattering process anymore, this step should be reconsidered.

Open nitrogen in addition to indium and watch the RHEED intensity. At 
some point the intensity should go down. The Ga adlayer is now gone. If you now open Ga, InGaN starts to grow. After InGaN growth, first close only N. After some time close In and close Ga a little bit later. This ensures that the protective layer is present at all times, even if In should desorp quickly. Afterwards, the sample is heated up to GaN growth temperature. During this time the Ga adlayer must not fully desorp. Check this by RHEED and deposit additional Ga if necessary. If a RHEED reconstruction is observed during the hole InGaN growth process, the sample is done for.

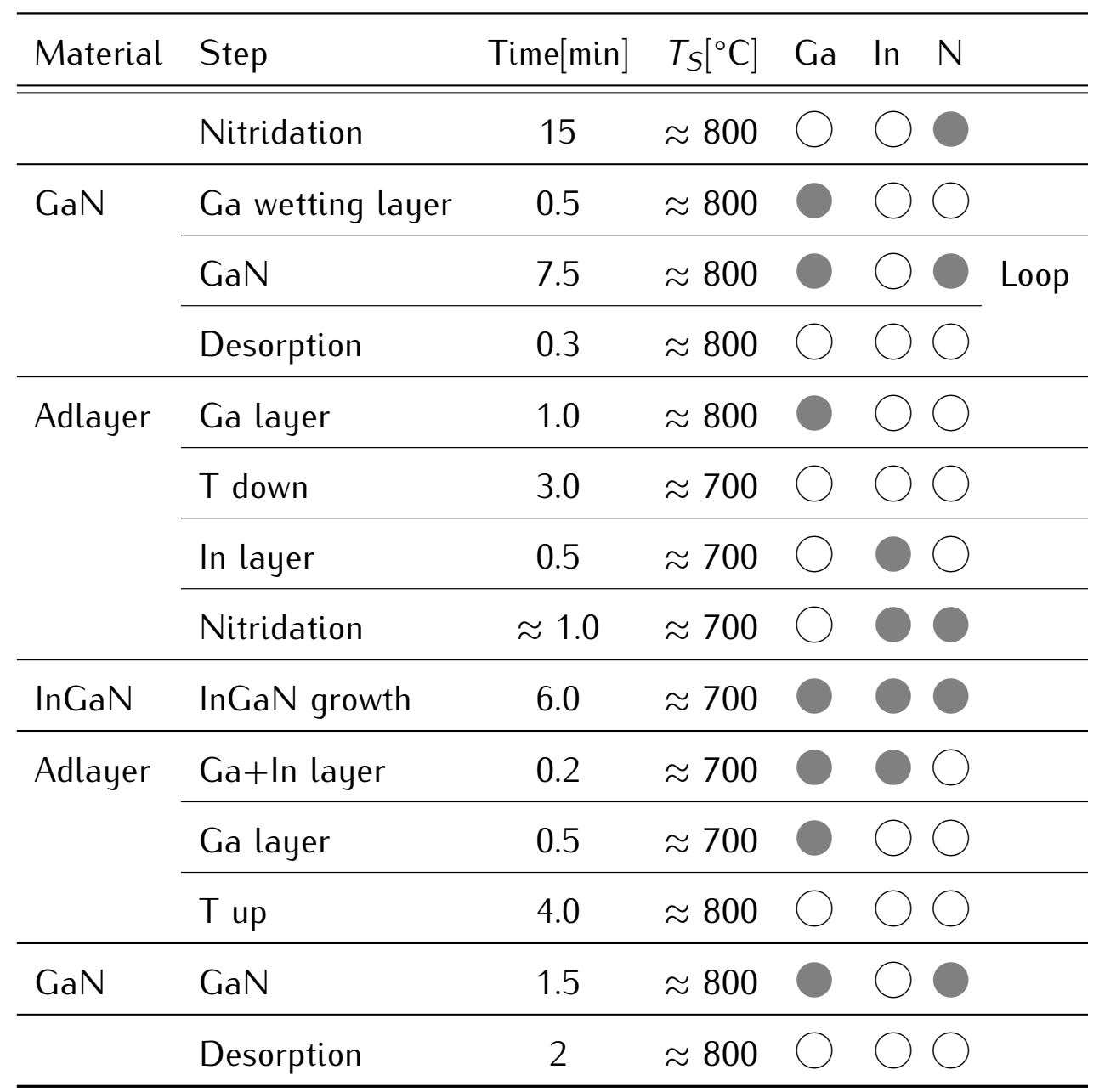

Table A.3: Recipe for GaN/InGaN/GaN growth 

RHEED 101

\section{B.1 THE HOMEBREW SOFTWARE}

The software used in our group is self written. I think it is working quite fine, but feel free to do further modifications if you want to increase the functionality or the stability. It is written in visual basic 6.0 and uses some external packages for the camera, the graphs and for live fitting of profiles. A little problem came up in the last year, when we upgraded the RHEED computer to Windows7. VB6 does not run well under Win7 and I tried to upgrade to .NET. This is complicated, as some packages are not compatible. You could program in the XP-mode, but the firewire camera does not work in this virtual system. I have to leave you with this problem, and suggest you program on another machine, if you cannot fix it.

The software works in the following way: An image is acquired from the camera. The image data is then passed to several classes that process the data. Only after all desired processes are completed, the next image is taken from the camera and the cycle starts over. The frames processed per second is therefore mainly limited by the amount of processing done at the same time.

If you start the software you will find a toolbar at the top of the window which is shown in Figure B.1. Some buttons are disabled, and will only be enabled as it makes sense, e.g. the intensity can only be saved if the data is processed. In the following, I will just describe some options and leave it

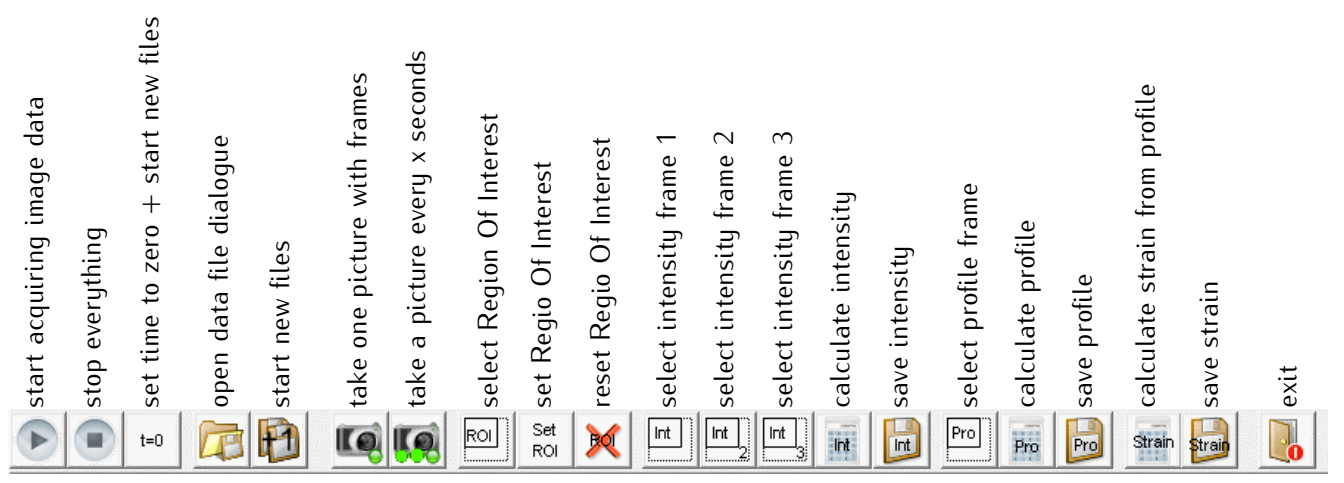

Figure B.1: RHEED Software Toolbar 
up to you to try the rest.

By pressing play, the images from the camera are acquired. You can change the size of the image by choosing a Region Of Interest (ROI). There are two buttons to take pictures. One will immediately save the picture on the screen, the other will take a series of pictures. You can place three intensity frames, from which the sum of the intensity can be plotted over time. You can place one profile frame, which gives you the sum of the intensity along the width or heigth of the frame. If you want to process the spacing between reflexes, you can choose strain. This will allow you to place two regions in the profile window. Within each region, the software looks for a maximum and fits a peak to it. It then gives you the distance between the two peaks in pixel. This allows to measure the distance between the two peaks with a sub-pixel accuracy.

Before you can save data or images you have to open the file dialogue. Here you can create or choose a directory. After doing so, always press change working directory. You can change the filenames individually or change the sample name and press change. The data you save will never be overwritten. As soon as you press one of the save buttons, the data will be saved in the file you specified. If you press $t=0$ the time will be set to zero and a new file will be created.

On the right hand side of the main window you will find a sidebar. Here you can switch on a noise filter, which takes the average over an amount of frames in order to decrease the noise signal. By clicking on device, you can access the camera options, in case you have lost the connection. The settings button, opens a dialogue for the camera setting as provided by the manufacturer. Here you can change important things like zoom, focus and exposure. In the sidebar you can define the time interval for image series and profile saving in seconds. The default is $10 \mathrm{~s}$. After you made a change, remember to press update. Under graphs, you will find a slider which changes the time scale of the intensity and strain graph. In the profile data section you can define whether the profile is taken along the width of the profile frame or along the height by selecting vertical of horizontal. It is also possible to substract a background in order to decreases the noise level in the profile data. You are also able to subtract a Gaussian profile, which is fitted to three or more points, by selecting filter. You can define the points in the profile window by pressing set. The last box in the sidebar is used to specify the peak finding in the profile window, in order to process the strain. By pressing set left max or set right max, you enable the positioning of a region for peak search in the profile window. You can change to interval within which the software looks for a peak. Remember not to choose the intervals to small, as the peak positions mighty change over growth time and leave the processed region.

So much for the software. The next section presents a short manual on how to acquire good data. 
First you have to turn on the electron gun. Do so, by switching the controller on and increase the voltage in $1 \mathrm{~V}$ steps and the current in $0.1 \mathrm{~A}$ steps every $10 \mathrm{~s}$ until you reach $14.5 \mathrm{~V}$ and $1.4 \mathrm{~A}$. This is close to the upper voltage and current limit of E-gun. These settings result in bright images. You can choose lower values, as long as the image stays bright enough.

The controller of the E-gun has five parameters: gain, focus, x-deflection, $y$-deflection and rocking angle. I cannot say which values are good for your measurement, so try around a little bit. I can however illustrate the process of getting a good image.

In order to adjust the beam, you first have to get the undiffracted spot onto the screen by changing $x$-deflection. If it is well focused it should look like the spot shown in Figure B.2. You can get a well focused spot by adjusting grind and focus. Afterwards, use $\mathrm{x}$ - and $\mathrm{y}$-deflection to move the spot onto the sample.

Now you have to rotate the same holder, in order to find the pattern you want to look at, which is usually one of the highly symmetric patterns. During rotation of the sample the intensity will change. You should compensate this by readjusting $x$ - and $y$-deflection to be able to compare the brightness of patterns that repeat every $60^{\circ}$. You should look for the brightest pattern you find. If you want to measure the strain, consider tilting the sample towards negative angles. This implies that the molecular beams will hit the sample under a non ideal angle, so keep the tilt small $\left(<3^{\circ}\right)$. By tilting the sample, the pattern moves deeper into the screen. Sounds strange, but you know what I mean when you see it.

If you found a pattern you like, use the y-deflection to check the impact of the sample holder prints. In some patterns you will see them moving through the image as shadows. Try to stay away from them. If you see them, you will probe a part of the sample which is very close to the holder and which is thus much colder than the rest of the sample. This might produce misleading results.

The rocking angle has to be adjusted as well. This is the inclination of the

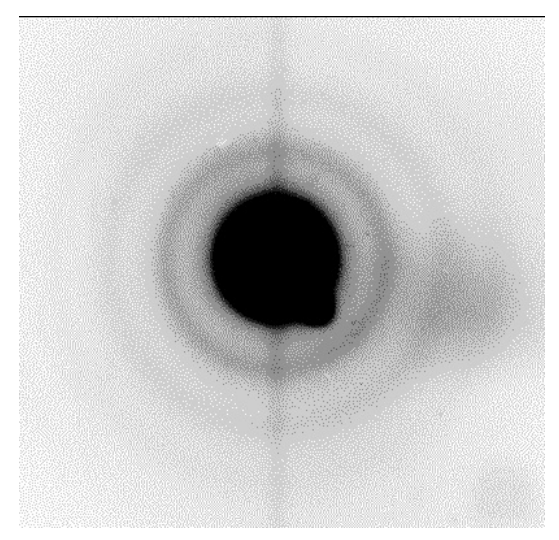

Figure B.2

Focused and undiffracted E-beam spot. Make sure the rings are as sharp as possible, by tuning grid and focus, before moving the spot onto the sample. 
E-beam onto your sample. If you want to be very surface sensitive, choose a small rocking angle. If you have trouble with artifacts from the sample holder, try to choose a larger angle. The angle also influence the area you probe with the beam. For smaller angles, the beam is in contact with the sample over longer distances. So if you choose a very small angle on a small sample, you will have large contributions from the edges of your sample. Therefore, I usually used intermediate angles.

Once you have a bright, symmetric pattern without artifacts of the holder, you can place the frames. The software allows you to place three intensity frames, and to get the most out of a measurement you should place all three. An example of how I used to place the intensity frames is shown in Figure B.3. I always placed two frames on the specular spot and one on a diffraction or reconstruction spot. The key to good intensity data is to know that you want to measure a change in the intensity. Therefore, do not place a frame on an area which is already at maximum intensity, as you probably see no reaction if the intensity increases or is decreased slightly. A frame that covers an area which has almost no signal compared to another area on the same reflex is not a good choice either, because it will show no change if the intensity is lowered. It is always good to try different frames and change them during a test run in order to see which selection results in the biggest intensity change. Do not vary the frames during measurement series like desorption studies, as you will not be able to compare the data at, e.g. the beginning and the end of your measurement.

In Figure B.3 a profile frame is shown as well. This is used to measure the distance between two reflexes. The upper part of the pattern is always a good choice. Here, the background signal from the specular spot is low, and the fitting procedure gives more reliable results.

This was just a guideline for you to get a good RHEED image. Always try to vary some of the parameters and see what works best for you.

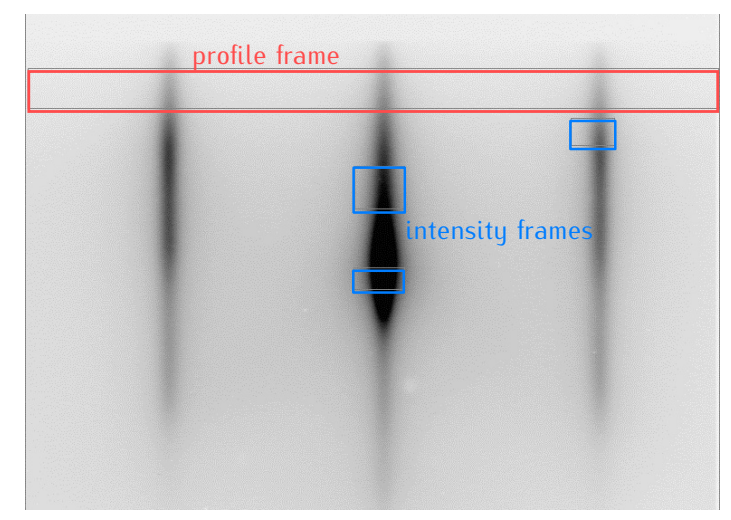

Figure B.3: Example of intensity and profile frames. Profile frame on to of your pattern, this minimizes the influence of the background. Intensity frames only partially on the specular spot, as it is to bright to monitor changes. Just make sure you have some signal left if the pattern gets very dark. 


\section{HOT OR NOT: ABSOLUTE SUBSTRATE TEMPERATURE}

The substrate temperature is a very important parameter in MBE growth. Differences of only a few degrees can change the growth conditions drastically. While the temperature can be controlled precisely, the absolute value of temperature is hard to access. This parameter becomes important when growing on different substrates and sample holders, and when comparing results to the literature or doing quantitative analysis. Usually the temperature in MBE is measured by pyrometers. This is not possible for our templates, which consist of GaN grown on sapphire. Both material are transparent for the wavelength range detected by the pyrometer and the temperature of the substrate cannot be probed by this technique. Even if a substrate suitable for pyrometric measurements is used, the temperature of the GaN surface will be different from the obtained value. This chapter therefore addresses the absolute temperature measurement of a GaN surface.

In the Veeco GEN II system used in this work the reading temperature gives the temperature of a resistance heater which is not in direct contact with the sample. The efficiency of the heat transmitted from the heater to the sample depends on various parameters, such as the size of the sample and the emission from the sample holder. The latter is very sensitive to the holder geometry, the coverage with material from previous growth cycles and even to small changes in the way a substrate is mounted.

The deviations of reading and absolute surface temperature are as high as $200^{\circ} \mathrm{C}$. In this chapter I present two methods to obtain the absolute temperature of the substrate. The first is based on RHEED and a pyrometric reference measurement and was used in this work. The temperature values obtained by this method compare well with temperatures reported in the literature for, e.g. GaN decomposition or optimum growth conditions for GaN. It is however not suited for in-situ temperature control. The second method is based on optical band edge absorption $[139,140]$. The setup for this technique is still under construction. 


\section{C.1 OLD SCHOOL: RHEED}

RHEED enables to get the absolute temperature right on the surface of a sample together with a reference measurement. The reference sample is measured with a pyrometer and is calibrated according to the melting point of aluminium deposited on the surface. In this work a template with $400 \mathrm{~nm}$ molybdenum deposited on the backside was used. A study of the desorption of gallium and indium is then used to assign the calibration to other samples.

With RHEED one can monitor the amount of metal on the surface and the state which it is in. For the calibration we will use the solid-liquid transition of aluminium at $660^{\circ} \mathrm{C}$. The first step of the calibration is to observe the melting of deposited Al. Grow some GaN first, in order to have a surface comparable to one you are usually working with. In principle, seeing Al melt is easy. However, in order to precisely measured it by RHEED, a series of steps has proven useful:

1. choose your RHEED spot to be in the middle of the sample (brightest when on $\left.1 \times 1 \mathrm{~cm}^{2}\right)$.

2. deposit $\mathrm{Al}$ at a low temperature $\left(T_{S}=300^{\circ} \mathrm{C}\right)$ for about $1 \mathrm{~min}$ with $T_{A l}=1025^{\circ} \mathrm{C}$ till you clearly observe Al reflexes as shown in Figure C.1.

3. heat up, while checking the substrate temperature and watch the $\mathrm{Al}$ reflexes with RHEED.

4. stop when you are sure that all Al reflexes are gone and cool down again till you are sure that you can see Al reflexes again. Go some $10^{\circ} \mathrm{C}$ further down.

5. place 2 intensity frames on the $\mathrm{Al}$ reflexes and one on GaN as a reference and the profile frame at the very top of the Al pattern to monitor the strain.

Figure C.1

RHEED pattern of a thin solid aluminium layer ontop of GaN (0001). The intensity of the aluminium reflexes decreases during aluminium melting.

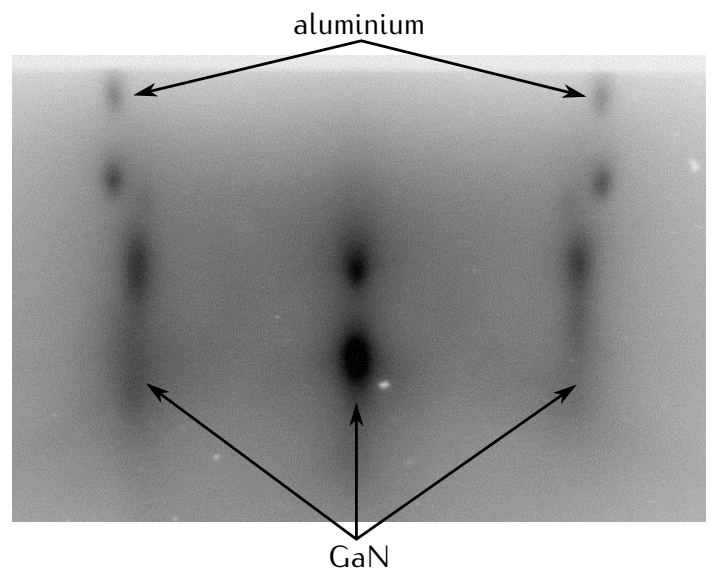




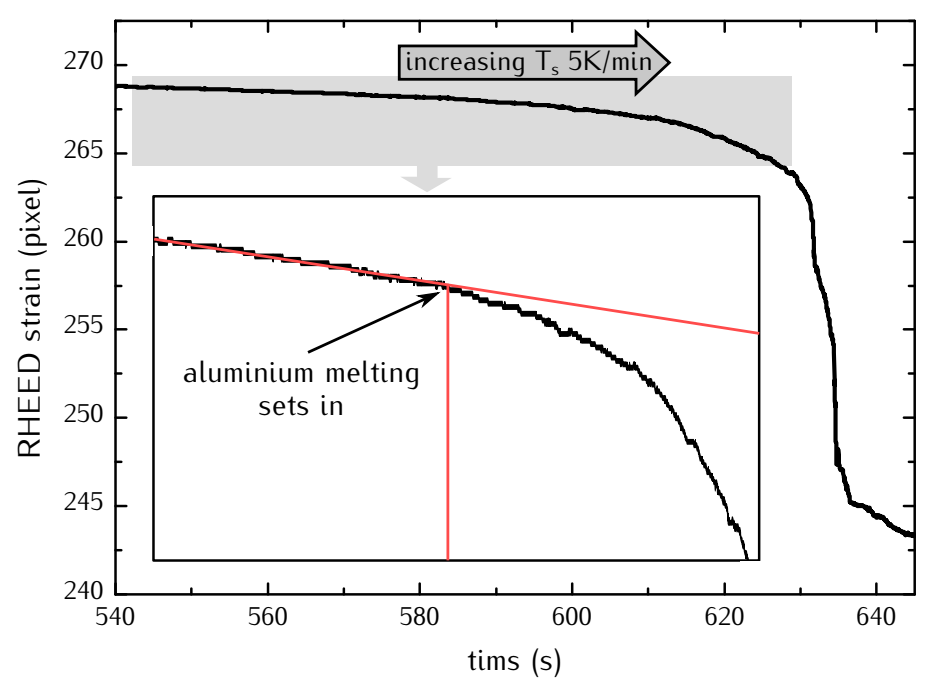

Figure C.2: RHEED strain signal over time during substrate heating with $5 \mathrm{~K} / \mathrm{min}$. The point when the trend does not follow the linear trend, which is observed because of the increasing temperature, gives the melting point of aluminium.

6. now heat up the substrate with a ramp no higher than $10 \mathrm{~K} / \mathrm{min}$, safe intensity and strain and write down the starting temperature at $t=0$.

7. your intensity and strain will change over time according to the temperature ramp chosen.

8. at some point you will observe a change in the slope of the intensity and the strain, but keep going just a little longer.

9. the recorded data should look like Figure C.2. You can now determine the time when the melting started and can derive the melting point using the starting temperature together with the temperature ramp.

This will also work with lowering the temperature, but the result will differ by around $20^{\circ} \mathrm{C}$. The reason is the lower temperature at the edges of the sample. With RHEED you can only precisely measure the first change of the signal. When heating up, the metal in the center of the sample will melt first, as this is the hottest area. When cooling down, however, crystallization on the edges will be monitored, as they are colder and will start to crystallize first.

The value of the Al melting is then used to calibrate the pyrometer. Set the substrate temperature to the value where you observed to melting of aluminium. Then change the emissivity value of the pyrometer until you obtain $660^{\circ} \mathrm{C}$. The pyrometer is now calibrated to the melting point of $\mathrm{Al}$ on the GaN surface.

We are not done yet. In order to use the calibration on other samples, some reference points are needed. You can obtain some, by monitoring the 


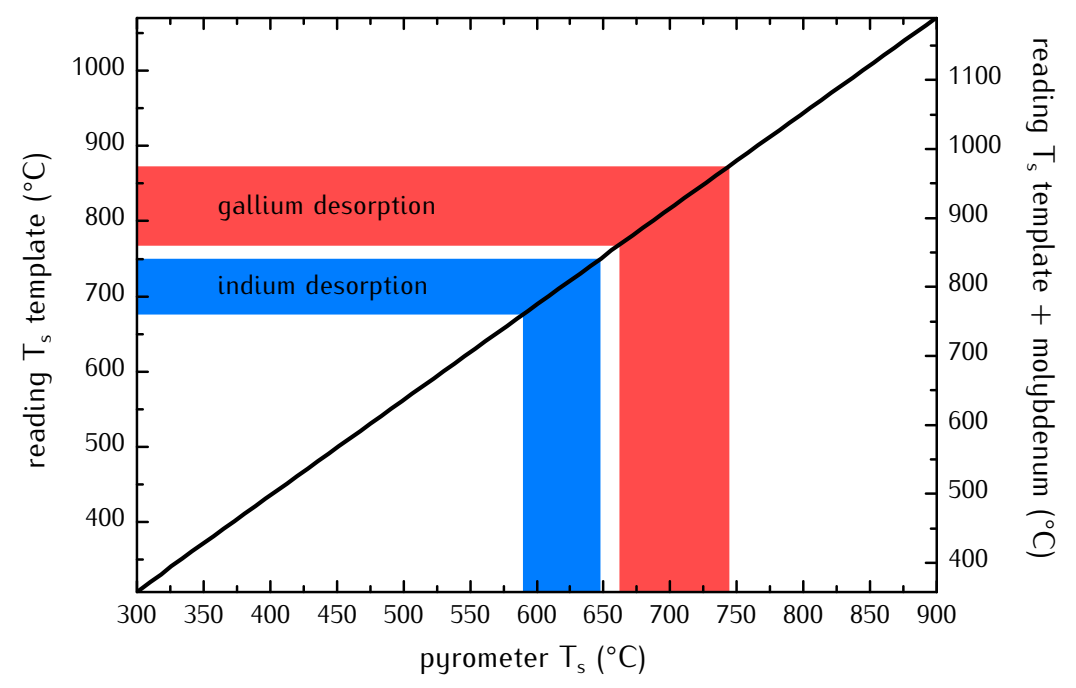

Figure C.3: Reading substrate temperature for a standard GaN template and a GaN template with a $400 \mathrm{~nm}$ molybdenum backcoating over calibrated pyrometric temperature. Strong deviations between the absolute temperature measured by the pyrometer and the reading temperature are observed. The temperature range for desorption of indium and gallium is indicated.

desorption time of Ga and In at various calibrated temperatures. Choose a certain flux and write down the desorption time and the corresponding calibrated pyrometer temperature. By repeating this desorption studies on other sample holders and on substrate without back coating, the temperature calibration can be assigned to other sample holders or substrates.

The temperature calibration used in this work is shown in Figure C.3. It gives the reading temperature of a standard GaN template and a molybdenum back coated template over the calibrated pyrometric temperature. You notice, that the reading temperature is far higher than the absolute temperature. If you have not done this calibration for your sample holder or substrate, bare this in mind during discussions and while reading literature. The figure also indicates the temperature range for the desorption time measurements.

\section{C.2 NEW SCHOOL: BAND EDGE ABSORPTION}

Band edge absorption is a more precise way of measuring the absolute temperature and can also be applied in-situ. The basic setup of this technique in $\mathrm{MBE}$ is shown in Figure C.4. Light shines onto the sample and the diffuse reflected light is analyzed by a spectrometer.

The spectrum of the reflected light will show an absorption edge as depicted in Figure C.5 a). Light with energies above the band gap will be absorbed, while light with lower energies will be reflected. The band gap can thus be determined by this spectrum. As the band gap energy depends on temperature, 


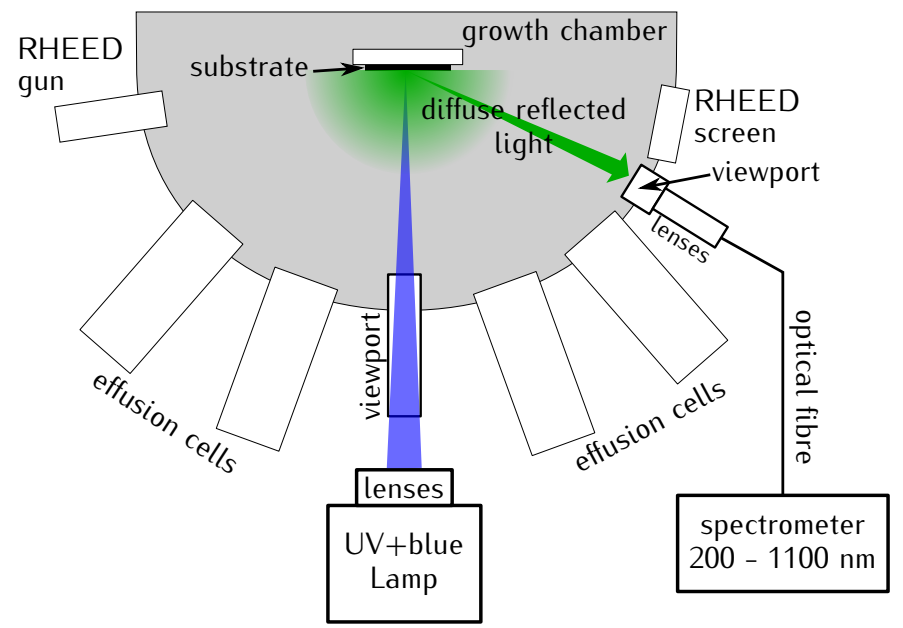

\section{Figure C.4}

Setup for band edge absorption measurement. Light is focused onto the sample through a viewport. The diffuse reflected light gets detected via a viewport which is not affected by directly reflected light. The signal is then analyzed by a spectrometer.

as shown in Figure C.5 b) for wurtzite GaN, so does the spectrum of the reflected light. This temperature dependence can either be taken from the literature or it may be calibrated.

This setup is still under construction in our MBE system. The spectrometer and the optics to collect diffuse reflected light are already installed. Example measurement with an UV lamp for lithography showed, that for our templates a wavelength between $380 \mathrm{~nm}$ and $450 \mathrm{~nm}$ has to analyzed. However, a lithography lamp is not designed for long exposures. At this stage, we are still looking for an affordable lamp with the desired wavelength range which is bright enough to produce good signals in the spectrometer.
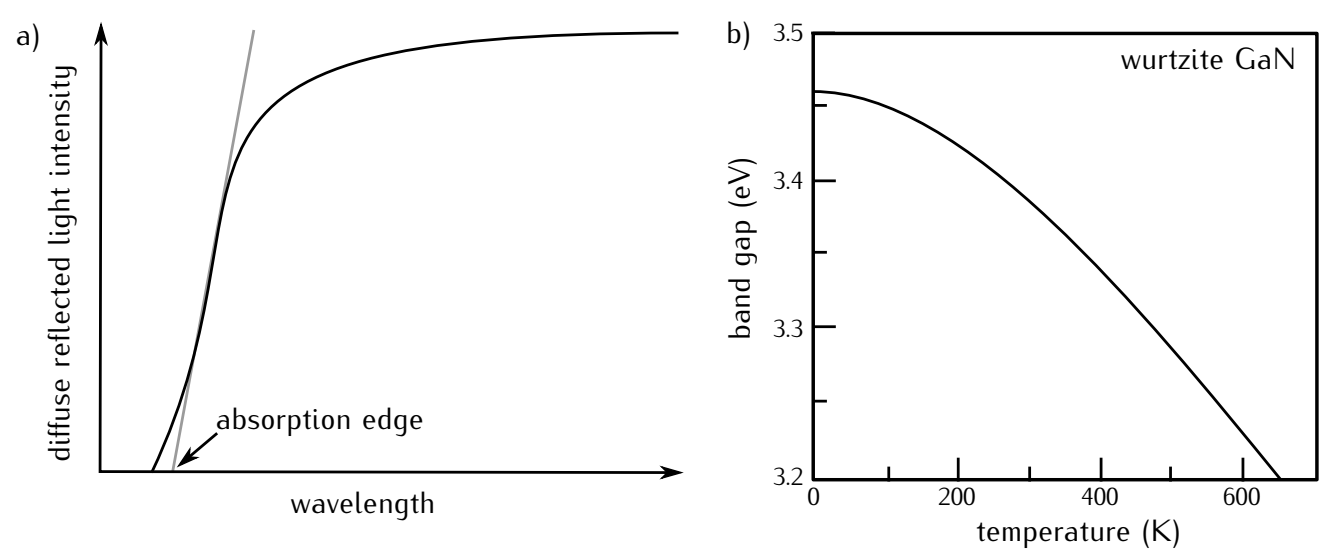

Figure C.5: a) illustrated data for an absorption edge measurement: intensity of the diffuse reflected over wavelength. b) band gap of wurtzite GaN over temperature [141]. 



\section{ACKNOWLEDGMENTS}

As this work ends, a new phase of my live starts. From former student to future employee. From former bachelor to future father. I would like to take this opportunity to thank some people who I had the privilege to work, live and party with.

First of all I would like to thank Prof. Dr. Angela Rizzi and Dr. Jörg Malindretos for their great support through thick and thin phases of my work in the past four years. I really appreciated their time and patience during long and fruitful discussions. Without them this work would not have been possible.

Thanks to Prof. Dr. Andreas Hangleiter, for finding the time co-refereeing my work and to be there for my defense.

Cheers to my Aussie mates from Perth. It was a pleasure working with you Anna Podolska, Brett Nener, Giacinta Parish and Gilberto UmanaMembreno. Good on ya.

I owe sincere thankfulness to Murat Sivis, Christian Zube and Arne Urban for the time they spend in the lab, analyzing my samples.

Prost to all my other fellow coworkers like Christan Denker, Amilcar Bedoya Pinto, Janna Emkes, Marco Bertelli, Dong Du Mai, Martin Röver, Boris Landgraph, Henning Hollermann, Emanuel Franke, Thomas Lehmann and everybody I forgot. You get to meet a lot of people spending six years in the same lab.

It was joy to work in the IV. Physik and I would like to thank all its members among them Rainer G. Ulbrich, Martin Wenderoth, Michael Seibt, Karl Ahlborn and Antje Spliethoff-Laiser.

I would also like to thank my mother Brigitte Broxtermann. Without her I would not be where I am today, as in living or in career. Thanks mum. Thanks to all my friends. Thanks to Thomas Rademacher, Britta \& Jakob Walowski, Karen \& Simon Hügelmeyer, Martin Kreusch, Moritz Ross and everybody else I had a chat or drink with during my time in Göttingen. Last but very not least I would like to thank Karen Teichmann who has been the joy of my life in the past years and was the motivator of this work at the homefront. Finally I would like to thank my soon to be born child, I love you. 

[1] R Juza and H Hahn. Über die Kristallstrukturen von Cu3N, GaN und InN Metallamide und Metallnitride. Z. Anorg. Allg. Chem, 239: 282-287, 1938.

[2] HP Maruska and JJ Tietjen. The preparation and properties of vapordeposited single-crystal GaN. Appl. Phys. Lett., 15(10):327, 1969.

[3] JI Pankove, EA Miller, and JE Berkeyhe. GaN electroluminescent diodes. R.C.A. Review, 32(3):383, 1971.

[4] S Yoshida, S Misawa, and S Gonda. Improvements on the electrical and luminescent properties of reactive molecular beam epitaxially grown GaN films by using AIN coated sapphire substrates. Appl. Phys. Lett., 42(5):427-429, 1983.

[5] H Amono, N Sakawi, I Anasaki, and Y Toyoda. Metalorganic vapor phase epitaxial growth of a high quality GaN film using an AIN buffer layer. Appl. Phys. Lett., 48(5):353-355, 1986.

[6] S Nakamura and T Mukai. High-quality InGaN films grown on GaN films. Jpn. J. Appl. Phys., Part 2, 31(10B):L1457-L1459, 1992.

[7] AY Cho. Morphology of epitaxial growth of GaAs by a molecular beam method: The observation of surface structures. J. Appl. Phys., 41(7): 2780, 1970.

[8] AY Cho. How molecular beam epitaxy (MBE) began and its projection into the future. J. Cryst. Growth, 201:1-7, 1999.

[9] H Morkoc. III-nitride semiconductor growth by MBE: Recent issues. J. Mater. Sci. - Mater. Electron., 12(12):677-695, 2001.

[10] H Morkoc. Handbook of Nitride Semiconductors and Devices, Volume 1: Materials Properties, Physics and Growth. WILEY-VCH, 2008.

[11] S Porowski. Growth and properties of single crystalline GaN substrates and homoepitaxial layers. Mater. Sci. Eng., B, 44(1-3):407-413, 1997.

[12] XQ Wang and A Yoshikawa. Molecular beam epitaxy growth of GaN, AIN and InN. Prog. Cryst. Growth Charact. Mater., 48-9:42-103, 2004. 
[13] D Billingsley, W Henderson, and WA Doolittle. High-temperature growth of GaN and AlGaN via ammonia-based metalorganic molecularbeam epitaxy. J. Electron. Mater., 39(5):473-477, 2010.

[14] XJ Ning, FR Chien, P Pirouz, JW Yang, and MA Khan. Growth defects in GaN films on sapphire: The probable origin of threading dislocations. J. Mater. Res., 11(3):580-592, 1996.

[15] A Sakai, H Sunakawa, and A Usui. Defect structure in selectively grown GaN films with low threading dislocation density. Appl. Phys. Lett., 71(16):2259-2261, 1997.

[16] Saint-Gobain Crystals formerly LUMILOG: http://www.photonic.saint gobain.com.

[17] K Jarasiunas, T Malinauskas, S Nargelas, V Gudelis, JV Vaitkus, $\checkmark$ Soukhoveev, and A Usikov. Layer thickness dependent carrier recombination rate in HVPE GaN. Phys. Status Solidi B, 247(7): 1703-1706, 2010.

[18] YY Wong, EY Chang, YH Wu, MK Hudait, TH Yang, JR Chang, JT Ku, WC Chou, CY Chen, JS Maa, and YC Lin. Dislocation reduction in GaN film using Ga-lean GaN buffer layer and migration enhanced epitaxy. Thin Solid Films, 519(19):6208-6213, 2011.

[19] Ammono S.A.: www.ammono.com.

[20] N Newman. The energetics of the GaN MBE reaction: A case study of meta-stable growth. J. Cryst. Growth, 178(1-2):102-112, 1997.

[21] G Bruno, M Losurdo, TC Kim, and A Brown. Adsorption and desorption kinetics of Ga on GaN(0001): Application of Wolkenstein theory. Phys. Rev. B: Condens. Matter, 82(7):075326, 2010.

[22] SC Jain, M Willander, J Narayan, and R Van Overstraeten. III-nitrides: Growth, characterization, and properties. J. Appl. Phys., 87(3):9651006, 2000.

[23] DD Koleske, AE Wickenden, RL Henry, WJ DeSisto, and RJ Gorman. Growth model for GaN with comparison to structural, optical, and electrical properties. J. Appl. Phys., 84(4):1998-2010, 1998.

[24] CS Gallinat, G Koblmueller, JS Brown, and JS Speck. A growth diagram for plasma-assisted molecular beam epitaxy of $\ln$-face $\ln N$. J. Appl. Phys., 102(6):064907, 2007.

[25] BL VanMil, HC Guo, LJ Holbert, K Lee, TH Myers, T Liu, and D Korakakis. High temperature limitations for GaN growth by rf-plasma 
assisted molecular beam epitaxy: Effects of active nitrogen species, surface polarity, hydrogen, and excess Ga-overpressure. J. Vac. Sci. Technol., B, 22(4):2149-2154, 2004.

[26] K Byrappa and T Ohachi. Crystal Growth Technology. Springer, 2003.

[27] IV Markov. Crystal growth for beginners: fundamentals of nucleation, crystal growth and epitaxy. World Scientific Publishing Company, 1995.

[28] A Ishizaka and Y Murata. Crystal growth model for molecular beam epitaxy: Role of kinks on crystal growth. J. Phys. Condens. Matter, 6 (45):L693-L698, 1994.

[29] B Heying, EJ Tarsa, CR Elsass, P Fini, SP DenBaars, and JS Speck. Dislocation mediated surface morphology of GaN. J. Appl. Phys., 85 (9):6470-6476, 1999.

[30] H Okumura, K Balakrishnan, H Hamaguchi, T Koizumi, S Chichibu, $\mathrm{H}$ Nakanishi, T Nagatomo, and S Yoshida. Analysis of MBE growth mode for GaN epilayers by RHEED. J. Cryst. Growth, 189:364-369, 1998.

[31] JE Northrup, J Neugebauer, RM Feenstra, and AR Smith. Structure of GaN(0001): The laterally contracted Ga bilayer model. Phys. Rev. B: Condens. Matter, 61(15):9932-9935, 2000.

[32] C Adelmann, J Brault, D Jalabert, P Gentile, H Mariette, G Mula, and B Daudin. Dynamically stable gallium surface coverages during plasma-assisted molecular-beam epitaxy of (0001) GaN. J. Appl. Phys., 91(12):9638-9645, 2002.

[33] J Neugebauer, TK Zywietz, M Scheffler, JE Northrup, H Chen, and RM Feenstra. Adatom kinetics on and below the surface: The existence of a new diffusion channel. Phys. Rev. Lett., 90(5):056101, 2003.

[34] RC Schoonmaker, A Buhl, and J Lemley. Vaporization catalysis. The decomposition of gallium nitride. J. Phys. Chem., 69(10):3455, 1965.

[35] C Skierbiszewski, ZR Wasilewski, I Grzegory, and S Porowski. Nitridebased laser diodes by plasma-assisted MBE-from violet to green emission. J. Cryst. Growth, 311:1632-1639, 2009.

[36] L Liu and JH Edgar. Substrates for gallium nitride epitaxy. Mater. Sci. Eng., R, 37(3):61-127, 2002.

[37] N Grandjean, J Massies, and M Leroux. Nitridation of sapphire. effect on the optical properties of GaN epitaxial overlayers. Appl. Phys. Lett., 69(14):2071-2073, 1996. 
[38] TD Moustakas, T Lei, and RJ Molnar. Growth of GaN by ECR-assisted MBE. Physica B, 185(1-4):36-49, 1993.

[39] G Koblmueller, F Wu, T Mates, JS Speck, S Fernandez-Garrido, and E Calleja. High electron mobility GaN grown under N-rich conditions by plasma-assisted molecular beam epitaxy. Appl. Phys. Lett., 91(22): 221905, 2007.

[40] M Leyer, J Stellmach, Ch Meissner, M Pristovsek, and M Kneissl. The critical thickness of InGaN on (0001)GaN. J. Cryst. Growth, 310(23): 4913-4915, 2008.

[41] B Heying, I Smorchkova, C Poblenz, C Elsass, P Fini, S Den Baars, U Mishra, and JS Speck. Optimization of the surface morphologies and electron mobilities in GaN grown by plasma-assisted molecular beam epitaxy. Appl. Phys. Lett., 77(18):2885-2887, 2000.

[42] C Adelmann, J Brault, G Mula, B Daudin, L Lymperakis, and J Neugebauer. Gallium adsorption on (0001) GaN surfaces. Phys. Rev. B: Condens. Matter, 67(16):165419, 2003.

[43] A Pisch and R Schmid-Fetzer. In situ decomposition study of GaN thin films. J. Cryst. Growth, 187(3-4):329-332, 1998.

[44] AR Arehart, C Poblenz, JS Speck, and SA Ringel. Effect of nitrogen plasma power on defect levels in Ni/n-GaN Schottky diodes grown by molecular beam epitaxy. J. Appl. Phys., 107(5):054518, 2010.

[45] C Skierbiszewski, Z Wasilewski, M Siekacz, A Feduniewicz, B Pastuszka, I Grzegory, M Leszczynski, and S Porowski. Growth optimisation of the GaN layers and GaN/AlGaN heterojunctions on bulk GaN substrates using plasma-assisted molecular beam epitaxy. Phys. Status Solidi A, 201(2):320-323, 2004.

[46] MJ Manfra, KW Baldwin, AM Sergent, KW West, RJ Molnar, and J Caissie. Electron mobility exceeding $160000 \mathrm{~cm}(2) / \mathrm{v}$ s in AlGaN/GaN heterostructures grown by molecular-beam epitaxy. Appl. Phys. Lett., 85(22):5394-5396, 2004.

[47] O Ambacher, B Foutz, J Smart, JR Shealy, NG Weimann, K Chu, M Murphy, AJ Sierakowski, WJ Schaff, LF Eastman, R Dimitrov, A Mitchell, and M Stutzmann. Two dimensional electron gases induced by spontaneous and piezoelectric polarization in undoped and doped AlGaN/GaN heterostructures. J. Appl. Phys., 87(1):334-344, 2000.

[48] E Iliopoulos and TD Moustakas. Growth kinetics of AlGaN films by plasma-assisted molecular-beam epitaxy. Appl. Phys. Lett., 81(2): 295-297, 2002. 
[49] P Waltereit, H Sato, C Poblenz, DS Green, JS Brown, M McLaurin, T Katona, SP DenBaars, JS Speck, JH Liang, M Kato, H Tamura, $\mathrm{S}$ Omori, and C Funaoka. Blue GaN-based light-emitting diodes grown by molecular-beam epitaxy with external quantum efficiency greater than 1.5\%. Appl. Phys. Lett., 84(15):2748-2750, 2004.

[50] C Poblenz, P Waltereit, and JS Speck. Uniformity and control of surface morphology during growth of GaN by molecular beam epitaxy. J. Vac. Sci. Technol., B, 23(4):1379-1385, 2005.

[51] VG Deibuk and AV Voznyi. Thermodynamic stability and redistribution of charges in ternary AlGaN, InGaN, and InAIN alloys. J. Semicond., 39(6):623-628, 2005.

[52] E Iliopoulos, KF Ludwig, TD Moustakas, and SNG Chu. Chemical ordering in AlGaN alloys grown by molecular beam epitaxy. Appl. Phys. Lett., 78(4):463-465, 2001.

[53] P Ruterana, GD Jores, M Laugt, F Omnes, and E Bellet-Amalric. Evidence for multiple chemical ordering in AlGaN grown by metalorganic chemical vapor deposition. Appl. Phys. Lett., 78(3):344-346, 2001.

[54] C Adelmann, R Langer, G Feuillet, and B Daudin. Indium incorporation during the growth of InGaN by molecular-beam epitaxy studied by reflection high-energy electron diffraction intensity oscillations. Appl. Phys. Lett., 75(22):3518-3520, 1999.

[55] M Moseley, J Lowder, D Billingsley, and WA Doolittle. Control of surface adatom kinetics for the growth of high-indium content InGaN throughout the miscibility gap. Appl. Phys. Lett., 97(19):191902, 2010.

[56] SM Bedair, FG Mclntosh, JC Roberts, EL Piner, KS Boutros, and NA EIMasry. Growth and characterization of In-based nitride compounds. J. Cryst. Growth, 178(1-2):32-44, 1997.

[57] C Skierbiszewski, P Perlin, I Grzegory, ZR Wasilewski, M Siekacz, A Feduniewicz, P Wisniewski, J Borysiuk, P Prystawko, G Kamler, T Suski, and S Porowski. High power blue-violet InGaN laser diodes grown on bulk GaN substrates by plasma-assisted molecular beam epitaxy. Semicond. Sci. Technol., 20(8):809-813, 2005.

[58] M Siekacz, A Feduniewicz-Zmuda, G Cywinski, M Krysko, I Grzegory, S Krukowski, KE Waldrip, W Jantsch, ZR Wasilewski, S Porowski, and C Skierbiszewski. Growth of InGaN and GaN/InGaN quantum wells by plasma-assisted molecular beam epitaxy. J. Cryst. Growth, 310(17): 3983-3986, 2008. 
[59] A Das, S Magalhaes, Y Kotsar, PK Kandaswamy, B Gayral, K Lorenz, $E$ Alves, $P$ Ruterana, and E Monroy. Indium kinetics during the plasma-assisted molecular beam epitaxy of semipolar (11-22) InGaN layers. Appl. Phys. Lett., 96(18):181907, 2010.

[60] SY Karpov. Suppression of phase separation in InGaN due to elastic strain. MRS Internet J. Nitride Semicond. Res., 3(16):16, 1998.

[61] CJ Humphreys. Does In form In-rich clusters in InGaN quantum wells? Philos. Mag., 87(13):1971-1982, 2007.

[62] SY Karpov and YN Makarov. Surface segregation in group-III nitride MBE. Phys. Status Solidi A, 188(2):611-614, 2001.

[63] MH Xie, J Zhang, A Lees, JM Fernandez, and BA Joyce. Surface segregation during molecular beam epitaxy: The site-blocking effects of surfactant atoms. Surf. Sci., 367(2):231-237, 1996.

[64] HJ Chen, RM Feenstra, JE Northrup, T Zywietz, J Neugebauer, and DW Greve. Surface structures and growth kinetics of InGaN(0001) grown by molecular beam epitaxy. J. Vac. Sci. Technol., B, 18(4): 2284-2289, 2000.

[65] TP Bartel, P Specht, JC Ho, and C Kisielowski. Phase separation in InGaN. Philos. Mag., 87(13):1983-1998, 2007.

[66] GB Stringfellow. Microstructures produced during the epitaxial growth of InGaN alloys. J. Cryst. Growth, 312(6):735-749, 2010.

[67] W Braun. Applied RHEED: reflection high-energy electron diffraction during crystal growth. Springer, 1999.

[68] V Ichimiya and PI Cohen. Reflection high-energy electron diffraction. Cambridge University Press, 2004.

[69] ZL Wang. Electron multiple inelastic scattering in the geometry of RHEED. Ultramicroscopy, 26(3):321-326, 1988.

[70] F Tang, T Parker, G-C Wang, and T-M Lu. Surface texture evolution of polycrystalline and nanostructured films: RHEED surface pole figure analysis. J. Phys. D-Appl. Phys., 40(23):R427-R439, 2007.

[71] K Miki, K Sakamoto, and T Sakamoto. Surface preparation of Si substrates for epitaxial growth. Surf. Sci., 406(1-3):312-327, 1998.

[72] J Suda, K Miura, M Honaga, Y Nishi, N Onojima, and H Matsunami. Effects of $6 \mathrm{H}-\mathrm{SiC}$ surface reconstruction on lattice relaxation of AIN buffer layers in molecular-beam epitaxial growth of GaN. Appl. Phys. Lett., 81(27):5141-5143, 2002. 
[73] E Lu, DC Ingram, AR Smith, JW Knepper, and FY Yang. Reconstruction control of magnetic properties during epitaxial growth of ferromagnetic Mn3-delta Ga on wurtzite GaN(0001). Phys. Rev. Lett., 97(14):146101, 2006.

[74] AR Smith, RM Feenstra, DW Greve, MS Shin, M Skowronski, J Neugebauer, and JE Northrup. Determination of wurtzite GaN lattice polarity based on surface reconstruction. Appl. Phys. Lett., 72(17):2114-2116, 1998.

[75] Y Kotsar, PK Kandaswamy, A Das, E Sarigiannidou, E Bellet-Amalric, and $\mathrm{E}$ Monroy. Strain relaxation in $\mathrm{GaN} / \mathrm{Al}(0.1) \mathrm{Ga}(0.9) \mathrm{N}$ superlattices for mid-infrared intersubband absorption. J. Cryst. Growth, 323(1, SI): 64-67, 2011.

[76] C Zube. MnGa/GaN-Heterostrukturen in Hinblick auf Spininjektion. Master's thesis, Georg-August-Universität Göttingen, 2010.

[77] F Bernardini, V Fiorentini, and D Vanderbilt. Spontaneous polarization and piezoelectric constants of III-V nitrides. Phys. Rev. B: Condens. Matter, 56(16):10024-10027, 1997.

[78] R Resta. Macroscopic polarization in crystalline dielectrics: the geometric phase approach. Rev. Mod. Phys., 66(3):899-915, 1994.

[79] O Ambacher, J Majewski, C Miskys, A Link, M Hermann, M Eickhoff, M Stutzmann, F Bernardini, V Fiorentini, V Tilak, B Schaff, and LF Eastman. Pyroelectric properties of $\mathrm{Al}(\mathrm{In}) \mathrm{GaN} / \mathrm{GaN}$ hetero- and quantum well structures. J. Phys. Condens. Matter, 14(13):3399-3434, 2002.

[80] F Bernardini and V Fiorentini. Nonlinear macroscopic polarization in III-V nitride alloys. Phys. Rev. B: Condens. Matter, 64(8):085207, 2001.

[81] CG Van de Walle and J Neugebauer. First-principles calculations for defects and impurities: Applications to III-nitrides. J. Appl. Phys., 95 (8):3851-3879, 2004.

[82] M Kocan, A Rizzi, H Luth, S Keller, and UK Mishra. Surface potential at as-grown GaN(0001) MBE layers. Phys. Status Solidi B, 234(3): 773-777, 2002.

[83] Y Dong, RM Feenstra, and JE Northrup. Electronic states of oxidized GaN(0001) surfaces. Appl. Phys. Lett., 89(17):171920, 2006.

[84] BK Ridley, O Ambacher, and LF Eastman. The polarization-induced electron gas in a heterostructure. Semicond. Sci. Technol., 15(3): 270-271, 2000. 
[85] JP Ibbetson, PT Fini, KD Ness, SP DenBaars, JS Speck, and UK Mishra. Polarization effects, surface states, and the source of electrons in AlGaN/GaN heterostructure field effect transistors. Appl. Phys. Lett., 77(2):250-252, 2000.

[86] GE Stillman and CM Wolfe. Electrical characterization of epitaxial layers. Thin Solid Films, 31(1-2):69-88, 1976.

[87] MN Gurusinghe, SK Davidsson, and TG Andersson. Two-dimensional electron mobility limitation mechanisms in AlGaN/GaN heterostructures. Phys. Rev. B: Condens. Matter, 72(4):045316, 2005.

[88] MJ Manfra, KW Baldwin, AM Sergent, RJ Molnar, and J Caissie. Electron mobility in very low density GaN/AlGaN/GaN heterostructures. Appl. Phys. Lett., 85(10):1722-1724, 2004.

[89] NG Weimann, LF Eastman, D Doppalapudi, HM Ng, and TD Moustakas. Scattering of electrons at threading dislocations in GaN. J. Appl. Phys., 83(7):3656-3659, 1998.

[90] I Vurgaftman and JR Meyer. Band parameters for nitrogen-containing semiconductors. J. Appl. Phys., 94(6):3675-3696, 2003.

[91] L Hsu, RE Jones, SX Li, KM Yu, and W Walukiewicz. Electron mobility in inn and III-n alloys. J. Appl. Phys., 102(7):073705, 2007.

[92] S Syed, MJ Manfra, YJ Wang, RJ Molnar, and HL Stormer. Electron scattering in AlGaN/GaN structures. Appl. Phys. Lett., 84(9):15071509, 2004.

[93] G Koblmueller, RM Chu, A Raman, UK Mishra, and JS Speck. Hightemperature molecular beam epitaxial growth of AlGaN/GaN on GaN templates with reduced interface impurity levels. J. Appl. Phys., 107 (4):043527, 2010.

[94] TB Fehlberg, GA Umana-Membreno, BD Nener, G Parish, CS Gallinat, G Koblmuller, S Rajan, S Bernardis, and JS Speck. Characterisation of multiple carrier transport in indium nitride, grown by molecular beam epitaxy. Jpn. J. Appl. Phys. Part 2 - Lett. Express Lett., 45(37-41): L1090-L1092, 2006.

[95] CX Wang, K Tsubaki, N Kobayashi, T Makimoto, and N Maeda. Electron transport properties in AlGaN/InGaN/GaN double heterostructures grown by metalorganic vapor phase epitaxy. Appl. Phys. Lett., 84(13):2313-2315, 2004.

[96] LJ van der Pauw. A method of measuring specific resistivity and hall effect of discs of arbitrary shape. Philips Research Reports, 13:1, 1958. 
[97] M Sivis. Zweidimensionale Elektronengase in AlGaN/GaNHeterostrukturen. Master's thesis, Georg-August-Universität Göttingen, 2009.

[98] B Van Daele, G Van Tendeloo, W Ruythooren, J Derluyn, MR Leys, and $\mathrm{M}$ Germain. The role of $\mathrm{Al}$ on ohmic contact formation on n-type GaN and AlGaN/GaN. Appl. Phys. Lett., 87:061905, 2005.

[99] B Van Daele, G Van Tendeloo, J Derluyn, P Shrivastava, A Lorenz, MR Leys, and M Germain. Mechanism for ohmic contact formation on Si3N4 passivated AlGaN/GaN high-electron-mobility transistors. Appl. Phys. Lett., 89(20):201908, 2006.

[100] T Ihn. Semiconductor Nanostructures: Quantum States and Electronic Transport. Oxford University Press, 2010.

[101] S Datta. Electronic Transport in Mesoscopic Systems. Cambridge University Press, 1997.

[102] T Heinzel. Mesoscopic Electronics in Solid State Nanostructures. Wiley-VCH, 2006.

[103] T Schäpers. Transport in Nanostructures (Script WiSe 2004/2005). RWTH Aachen, 2004.

[104] T Ando, AB Fowler, and F Stern. Electronic properties of twodimensional systems. Rev. Mod. Phys., 54(2):437-672, 1982.

[105] T Wang, J Bai, S Sakai, Y Ohno, and H Ohno. Magnetotransport studies of AlGaN/GaN heterostructures grown on sapphire substrates: Effective mass and scattering time. Appl. Phys. Lett., 76(19):2737-2739, 2000 .

[106] D Jena and UK Mishra. Quantum and classical scattering times due to charged dislocations in an impure electron gas. Phys. Rev. B: Condens. Matter, 66(24):241307, 2002.

[107] L Hsu and W Walukiewicz. Transport-to-quantum lifetime ratios in AlGaN/GaN heterostructures. Appl. Phys. Lett., 80(14):2508-2510, 2002.

[108] G Bergmann. Weak localization in thin films a time-of-flight experiment with conduction electrons. Phys. Rep., 107(1):1-58, 1984.

[109] GM Minkov, AV Germanenko, OE Rut, AA Sherstobitov, and BN Zvonkov. Disorder and temperature renormalization of interaction contribution to the conductivity in two-dimensional InGaAs electron systems. Phys. Rev. B: Condens. Matter, 79(23):235335, 2009. 
[110] DF Sullivan. The low temperature magnetoconductance of delta-dopes silicon. PhD thesis, University of Maryland, 2005.

[111] JJ Lin and JP Bird. Recent experimental studies of electron dephasing in metal and semiconductor mesoscopic structures. J. Phys. Condens. Matter, 14(18):R501-R596, 2002.

[112] H Fukuyama and E Abrahams. Inelastic scattering time in twodimensional disordered metals. Phys. Rev. B: Condens. Matter, 27 (10):5976-5980, 1983.

[113] A Dmitriev, M Dyakonov, and R Jullien. Classical mechanism for negative magnetoresistance in two dimensions. Phys. Rev. B: Condens. Matter, 64(23):233321, 2001.

[114] DG Polyakov, F Evers, AD Mirlin, and P Wolfle. Quasiclassical magnetotransport in a random array of antidots. Phys. Rev. B: Condens. Matter, 64(20):205306, 2001.

[115] VV Cheianov, AP Dmitriev, and VY Kachorovskii. Anomalous negative magnetoresistance caused by non-Markovian effects. Phys. Rev. B: Condens. Matter, 68(20):201304, 2003.

[116] VV Cheianov, AP Dmitriev, and VY Kachorovskii. Non-Markovian effects on the two-dimensional magnetotransport: Low-field anomaly in magnetoresistance. Phys. Rev. B: Condens. Matter, 70(24):245307, 2004.

[117] J Osaka, M Senthil Kumar, H Toyoda, T Ishijima, H Sugai, and T Mizutani. Role of atomic nitrogen during GaN growth by plasma-assisted molecular beam epitaxy revealed by appearance mass spectrometry. Appl. Phys. Lett., 90(17):172114, 2007.

[118] H Hollermann. GaN-MBE growth under different $\mathrm{N}$-plasma source conditions. Master's thesis, Georg-August-Universität Göttingen, 2010.

[119] E Franke. Entwicklung einer optischen Prozesskontrolleinheit für Stickstoffplasmaquellen. Master's thesis, Georg-August-Universität Göttingen, 2009.

[120] CG Van de Walle, MD McCluskey, CP Master, LT Romano, and NM Johnson. Large and composition-dependent band gap bowing in InGaN alloys. Mater. Sci. Eng., B, 59(1-3):274-278, 1999.

[121] FM Morales, D Gonzalez, JG Lozano, R Garcia, S Hauguth-Frank, $\checkmark$ Lebedev, $V$ Cimalla, and O Ambacher. Determination of the composition of InGaN from strain measurements. Acta Mater., 57(19): 5681-5692, 2009. 
[122] L Hsu and W Walukiewicz. Effect of polarization fields on transport properties in AlGaN/GaN heterostructures. J. Appl. Phys., 89(3): 1783-1789, 2001.

[123] nextnano3:

http://www.wsi.tum.de/nextnano3 and http://www.nextnano.de.

[124] X Han, Y Honda, T Narita, M Yamaguchi, and N Sawaki. Transport properties of the two-dimensional electron gas in AlGaN/GaN heterostructures. J. Phys. Condens. Matter, 19(4):046204, 2007.

[125] N Tang, B Shen, ZW Zheng, J Liu, DJ Chen, J Lu, R Zhang, Y Shi, YD Zheng, YS Gui, CP Jiang, ZJ Qiu, SL Guo, JH Chu, K Hoshino, $\mathrm{T}$ Someya, and $\mathrm{Y}$ Arakawa. Magnetoresistance oscillations induced by intersubband scattering of two-dimensional electron gas in $\mathrm{Al}(0.22) \mathrm{Ga}(0.78) \mathrm{N} / \mathrm{GaN}$ heterostructures. J. Appl. Phys., 94(8): 5420-5422, 2003.

[126] CP Jiang, SL Guo, ZM Huang, J Yu, YS Gui, GZ Zheng, JH Chu, ZW Zheng, B Shen, and YD Zheng. Subband electron properties of modulation-doped AlGaN/GaN heterostructures with different barrier thicknesses. Appl. Phys. Lett., 79:374, 2001.

[127] J Antoszewski, JM Dell, L Faraone, LS Tan, A Raman, SJ Chua, DS Holmes, JR Lindemuth, and JR Meyer. Evaluation of III-V multilayer transport parameters using quantitative mobility spectrum analysis. Mater. Sci. Eng., B, 44(1-3):65-69, 1997.

[128] E Bellotti, F Bertazzi, and M Goano. Alloy scattering in AlGaN and InGaN: A numerical study. J. Appl. Phys., 101(12):123706, 2007.

[129] A Podolska, M Kocan, AMG Cabezas, TD Wilson, GA UmanaMembreno, BD Nener, G Parish, S Keller, and UK Mishra. Ion versus $\mathrm{pH}$ sensitivity of ungated AlGaN/GaN heterostructure-based devices. Appl. Phys. Lett., 97(1):012108, 2010.

[130] A Podolska, D Broxtermann, J Malindretos, GA Umana-Membreno, S Keller, UK Mishra, A Rizzi, BD Nenner, and G Parish. Invesitgations into improved sensitivity of ungated $\mathrm{AlGaN} / \mathrm{GaN}$-based ion sensors. unpublished, 2011.

[131] V Cimalla, J Pezoldt, and O Ambacher. Group III nitride and SiC based MEMS and NEMS: materials properties, technology and applications. J. Phys. D: Appl. Phys., 40(20):6386-6434, 2007.

[132] BS Kang, HT Wang, F Ren, and SJ Pearton. Electrical detection of biomaterials using AlGaN/GaN high electron mobility transistors. J. Appl. Phys., 104(3):031101, 2008. 
[133] A Podolska, R Seeber, M Kocan, K Pfleger, G Parish, and BD Nener. Cell growth and attachment to AlGaN surfaces for biosensor applications. Proc. ICONN 2010, in press.

[134] H Morkoc. Handbook of Nitride Semiconductors and Devices, Volume 2: Electronic and Optical Processes in Nitrides. WILEY-VCH, 2008.

[135] MA Pinault and E Tournie. On the origin of carrier localization in GalnNAs/GaAs quantum wells. Appl. Phys. Lett., 78(11):1562-1564, 2001.

[136] WZ Zhou, T Lin, LY Shang, G Yu, K Han, JX Duan, N Tang, B Shen, and JH Chu. Diffusion and ballistic contributions of electron-electron interaction to the conductivity in an $\mathrm{Al}(0.26) \mathrm{Ga}(0.74) \mathrm{N} / \mathrm{AIN} / \mathrm{GaN}$ heterostructure. Solid State Commun., 151(12):879-882, 2011.

[137] A Washburn and RA Webb. Aharonov-bohm effect in normal metal quantum coherence and transport. Adv. Phys., 35:375-422, 1986.

[138] A Washburn and RA Webb. Quantum transport in small disordered samples from the diffusive to the ballistic regime. Rep. Prog. Phys., 55:1311, 1992.

[139] I Farrer, JJ Harris, R Thomson, D Barlett, CA Taylor, and DA Ritchie. Substrate temperature measurement using a commercial band-edge detection system. J. Cryst. Growth, 301-302:88-92, 2007.

[140] commercial solution by k-Space Associates Inc: www.k space.com/Products/BandiT.html.

[141] ME Levinshtein, SL Rumyantsev, and MS Shur. Properties of Advanced Semiconductor Materials: GaN, AIN, InN, BN, SiC, SiGe. WileyInterscience, 2001. 


\section{Daniel \\ Broxtermann}

Lebenslauf
Reinhäuser Landstr. 58

37083 Göttingen

Geburtsdatum:

Geburtsort:

Staatsangehrigkeit:
19.09.1980

Georgsmarienhütte

deutsch

\section{Schule}

07/1991- Kooperative Gesamtschule Osnabrück Schinkel

06/2000

Abschluss Allgemeine Hoschschulreife (Abitur)

\section{Zivildienst}

07/2000- Behindertenfahrdienst des Malteser Hilfsdienstes Osnabrück

$06 / 2001$

\section{Studium}

10/2001- Diplom Physik an der Universität Osnabrück (Vordiplom)

$09 / 2003$

10/2003- Diplom Physik an der Georg-August-Universität Göttingen

$07 / 2007$

Abschluss Diplom Physik

11/2007- Promotionstudium an der Georg-August-Universität Göttingen $10 / 2011$

\section{Diplomarbeit}

Insitut IV. Physikalisches Institut der Georg-August-Universität Göttingen

Arbeisgruppe Angela Rizzi

Titel "AlGaN/GaN Heterostrukturen hoher Beweglichkeit"

\section{Doktorarbeit}

Insitut IV. Physikalisches Institut der Georg-August-Universität Göttingen

Arbeisgruppe Angela Rizzi

Titel "Towards high electron mobility in GaN(0001) based InGaN and AlGaN heterostrucutres" 
